\title{
In Situ Infrared Spectroscopic Study of Iron-Catalyzed Hydromagnesiation of Vinyl Arenes
}

\author{
Jessica Ann Rogers \\ jroger27@mix.wvu.edu
}

Follow this and additional works at: https://researchrepository.wvu.edu/etd

Part of the Inorganic Chemistry Commons, and the Organic Chemistry Commons

\section{Recommended Citation}

Rogers, Jessica Ann, "In Situ Infrared Spectroscopic Study of Iron-Catalyzed Hydromagnesiation of Vinyl Arenes" (2019). Graduate Theses, Dissertations, and Problem Reports. 7432.

https://researchrepository.wvu.edu/etd/7432

This Dissertation is protected by copyright and/or related rights. It has been brought to you by the The Research Repository @ WVU with permission from the rights-holder(s). You are free to use this Dissertation in any way that is permitted by the copyright and related rights legislation that applies to your use. For other uses you must obtain permission from the rights-holder(s) directly, unless additional rights are indicated by a Creative Commons license in the record and/ or on the work itself. This Dissertation has been accepted for inclusion in WVU Graduate Theses, Dissertations, and Problem Reports collection by an authorized administrator of The Research Repository @ WVU.

For more information, please contact researchrepository@mail.wvu.edu. 
In Situ Infrared Spectroscopic Study of Iron-Catalyzed

Hydromagnesiation of Vinyl Arenes

\author{
Jessica A. Rogers \\ Dissertation submitted to the \\ Eberly College of Arts and Sciences \\ at West Virginia University \\ in partial fulfillment of the requirements for the \\ degree of
}

Doctor of Philosophy in Chemistry

Brian V. Popp, Ph. D., Committee Chair

Jessica M. Hoover, Ph.D.

Björn Söderberg, Ph.D.

Carsten Milsmann, Ph.D.

Mark McLaughlin, Ph.D.

Department of Chemistry

Morgantown, West Virginia 2019

Keywords: iron catalysis, hydromagnesiation, in situ spectroscopy, kinetic modeling 


\title{
Abstract \\ In Situ Infrared Spectroscopic Study of Iron-Catalyzed \\ Hydromagnesiation of Vinyl Arenes
}

\author{
Jessica A. Rogers
}

Iron catalysis, especially homogeneous catalysis, has been a resurging topic of organometallic chemistry research. Discussions of past and present mechanistic analyses for homogeneous iron catalysis will be discussed in Chapter 1. As an expansion of homogeneous iron catalysis, in situ infrared spectroscopy will be used to develop a full mechanistic study of iron-catalyzed hydromagnesiation of vinyl arenes. The kinetic analyses by both initial and observed rate measurements indicate complex concentration dependencies on the (PDI)iron catalyst as well as sacrificial Grignard reagent and styrene. These complexities are not limited to non-linear catalyst and Grignard initial rate and inhibition by styrene/Grignard at low concentrations which then change upon reaching concentrations of a $1: 1$ ratio by observed rates. The process of numeric timecourse simulation of probable mechanisms using COmplex PAthway Simulator (COPASI) led to the identification of a twelve-step mechanism that accurately reproduces experimental time course data over a wide variety of reaction conditions. ${ }^{1}$

This initial analysis was used as a building block for further identification of kinetic complexities when varying the electronics and sterics of the substrates and precatalysts. The development of an unexpected kinetic complexity with respect to the electronics of the styrene derivatives resulted in a strange "arrow-head" shaped Hammett correlation. DFT calculations and numeric timecourse simulation suggest a change in electronic character of a $p$-methoxystyrene or turnover limiting step, wherein the rate constants for the competitive 2,1- vs 1,2- insertion is significantly lower than that of transmetallation. Furthermore, predictions using the hydromagnesiation mechanism reveal the origins of the observed kinetic complexity. Identification of the limitations of some sacrificial Grignard reagents lead to the explanation of reaction complexity based upon the generation of a gaseous alkene byproduct. Following the kinetic analysis of Grignard reagents, electron-rich styrenes, and pre-catalysts bearing less sterically bulky PDI ligands led to the development of a mechanism consisting of 16 elementary steps. 
Dedicated to my parents, grandparents and brother, Jim (Zeke) and Sherri Rogers II

Jim and Nancy Rogers

\section{Don and Jan Martin}

John (J.J.) Rogers 


\section{Acknowledgements}

I would personally like to thank my advisor, Dr. Brian Popp. Without his help, I wouldn't be the young chemist I am today. I am thankful for his mentorship. He has taught and trained me to think outside the box on multiple occasions, thus allowing my research ideas in kinetic analysis to blossom into a beautiful, yet complicated mechanistic insight in the field of iron catalyzed hydrometallation. Dr. Popp is not only a wonderful mentor but a kind person with a wonderful family. His wife, Felicia, has always been kind and always knows just what to say. I wish him and his family nothing but the best in the future in all their endeavors.

I would also like to thank my other committee members, Dr. Jessica Hoover, Dr. Björn Söderberg, Dr. Carsten Milsmann, and Dr. Mark McLaughlin for taking the time to serve on my committee, as well as Dr. Werner Geldenhuys for stepping in during my oral defense. I greatly appreciate their advice and support throughout my tenure at WVU. I would like to thank Dr. Melissa Ely, Dr. Erin Battin, and Dr. Josh Osbourn for all their guidance in teaching. I would like to thank Dr. Mark Tinsley and Dr. Ken Showalter for answering my never-ending kinetics questions as I began my research journey. I would also like to say thank you to Becky Secrist, her willingness to drop anything to help myself and other graduate students makes her one in a million. She always wants the best for all of us. Furthermore, I would like to thank the staff for all of their help with maintaining instruments/equipment, Allan, Randy, and Sherman are truly wonderful.

Additionally, I would like to thank all the members of the Popp Group over the years. Thanks to Dr. Brian Nichols, Dr. Trina Perrone, Rachael Pickens, Notashia Baughman, Steven Knowlden, Randika Abeysinghe, and Alexa Martin. I would also like to thank our undergraduates as well, Trevor Butcher, Ted McClain, Tyler Hamilton, Amy Gregory, Maxwell Reese, Leandra Forte, Kayla Kroner, Natalie Ziemer, Tiffany Taylor, Mason Hamilton, Randy Koziel and Christopher Nau, they have made research fun. I wish each and every one of them happiness and all the best.

I would like to thank my family. Their unending love has kept me sane through my graduate school career. I want to thank Dad, Mom, Mamaw, Pappy, Papaw Don, JJ, Aunt Tammy (Mimmy), Uncle Bill, and Andrew for their never-ending thoughts, prayers, and for always wanting the best for me in life. They have taught me to be dedicated in everything I do, and to always do the right thing no matter what. Rob, thank you for always being there for me throughout our years in graduate school. I can't wait to see where life takes us. Lastly, I would like to thank my church family at Monongah Baptist Church for their unwavering love and support. 
Table of Contents

\begin{tabular}{|l|l|}
\hline Abstract & ii \\
\hline Dedication & ii \\
\hline Acknowledgements & iv \\
\hline Table of Contents & $\mathbf{v}$ \\
\hline List of Tables & vi \\
\hline List of Figures & vii \\
\hline List of Schemes & ix \\
\hline List of Abbreviations & x \\
\hline
\end{tabular}

Chapter 1. Introduction to Homogeneous Iron Catalysis

\begin{tabular}{|l|l|l|}
\hline 1.1 & Historical Milestones & 1 \\
\hline 1.2 & Iron and enzymes & 2 \\
\hline 1.3 & Iron-catalyzed cross coupling & 4 \\
\hline 1.4 & Iron-catalyzed polymerization & 9 \\
\hline 1.5 & Iron-catalyzed hydrogenation & 11 \\
\hline 1.6 & Iron-catalyzed hydro/hetero(element)functionalization & 15 \\
\hline 1.7 & Conclusions & 25 \\
\hline
\end{tabular}

Chapter 2.

\begin{tabular}{|l|l|l|}
\hline 2.1 & Introduction & 26 \\
\hline 2.2 & Results & 30 \\
\hline 2.3 & Conclusions & 58 \\
\hline
\end{tabular}

Chapter 3.

\begin{tabular}{|l|l|l|}
\hline 3.1 & Introduction & 59 \\
\hline 3.2 & Results & 60 \\
\hline 3.3 & Conclusions & 68 \\
\hline
\end{tabular}

Chapter 4.

\begin{tabular}{|l|l|l|}
\hline 4.1 & Introduction & 69 \\
\hline 4.2 & Results & 71 \\
\hline 4.3 & Conclusions & 79 \\
\hline
\end{tabular}

Chapter 5

\begin{tabular}{|l|l|l|}
\hline 5.1 & Introduction & 80 \\
\hline 5.2 & Results & 81 \\
\hline 5.3 & Conclusions & 88 \\
\hline
\end{tabular}

Conclusions

Experimental Appendix I

Experimental Appendix II (Tutorials) 


\section{List of Tables}

\begin{tabular}{|l|l|l|}
\hline 2.1 & Cohen's Kappa agreement definition & 34 \\
\hline 2.2 & $\begin{array}{l}\text { Calculated rate constants from COPASI for Scheme 2.2A direct } \beta \text {-hydride } \\
\text { transfer mechanism }\end{array}$ & 35 \\
\hline 2.3 & $\begin{array}{l}\text { Calculated rate constants from COPASI for Scheme 2.2A } \beta \text {-hydride } \\
\text { elimination/migratory insertion mechanism }\end{array}$ & 35 \\
\hline 2.4 & $\begin{array}{l}\text { Calculated rate constants from COPASI for Scheme 2.2B } \beta \text {-hydride } \\
\text { transfer 2019 mechanism }\end{array}$ & 35 \\
\hline 2.5 & Calculated rate constants from COPASI for Scheme 2.5A & 38 \\
\hline 2.6 & Calculated rate constants from COPASI for Scheme 2.5B & 40 \\
\hline 2.7 & Calculated rate constants from COPASI for Scheme 2.6A & 43 \\
\hline 2.8 & Calculated rate constants from COPASI for Scheme 2.6B & 45 \\
\hline 2.9 & Calculated rate constants from COPASI for Scheme 2.7 & 48 \\
\hline 2.10 & Calculated rate constants from COPASI for Scheme 2.8 & 52 \\
\hline 3.1 & Calculated rate constant ratio for styrene derivatives & 64 \\
\hline
\end{tabular}




\begin{tabular}{|c|c|c|}
\hline \multicolumn{3}{|c|}{ List of Figures } \\
\hline 1.1 & Iron Catalysis Time-line & 2 \\
\hline 1.2 & Iron-Heme enzyme catalysis & 3 \\
\hline 1.3 & Iron non-heme enzymes & 4 \\
\hline 1.4 & Common iron-catalyzed CC reactions. & 7 \\
\hline 1.5 & X-ray crystal structure of $\left(\mathrm{PDI} \mathrm{IPr}^{\mathrm{P}} \mathrm{FeCl}_{2}\right.$. & 9 \\
\hline 1.6 & Iron-Catalyzed hydrogenation catalysts. & 14 \\
\hline 1.7 & Iron-Catalyzed hydrosilylation side reactions & 17 \\
\hline 1.8 & Iron-Catalyzed hydrosilylation catalysts & 18 \\
\hline 1.9 & Iron-Catalyzed hydroboration catalysts & 19 \\
\hline 1.10 & Iron-Catalyzed hydroformylation catalysts & 20 \\
\hline 1.11 & Iron-Catalyzed formal hydrocarboxylation pathways & 22 \\
\hline 1.12 & Iron-Catalyzed difunctionalization pathways & 24 \\
\hline 2.1 & Synthesis of "Neoprofen" & 27 \\
\hline 2.2 & $\begin{array}{l}\text { Frequency analysis of the absorbances of the starting material and } \\
\text { the product from Scheme } 2.4 \text {. }\end{array}$ & 31 \\
\hline 2.3 & Reaction time courses & 32 \\
\hline 2.4 & $\begin{array}{l}\text { Concentration dependencies of initial and observed rates for } \\
\text { catalyst and substrates. }\end{array}$ & 33 \\
\hline 2.5 & Initial rate dependence of cyclopentene & 34 \\
\hline 2.6 & $\begin{array}{l}\text { Reaction time course and COPASI calculated time courses for } \\
\text { mechanisms in Scheme } 2.2\end{array}$ & 35 \\
\hline 2.7 & $\begin{array}{l}\text { Reaction time course and COPASI calculated time courses from } \\
\text { Scheme } 2.5 \mathrm{~A}\end{array}$ & 38 \\
\hline 2.8 & $\begin{array}{l}\text { Reaction time course and COPASI calculated time courses from } \\
\text { Scheme } 2.5 \mathrm{~A}\end{array}$ & 39 \\
\hline 2.9 & Lineweaver-Burk analysis of the Grignard dependence & 40 \\
\hline 2.10 & $\begin{array}{l}\text { Reaction time course and COPASI calculated time courses from } \\
\text { Scheme } 2.5 \mathrm{~B}\end{array}$ & 40 \\
\hline 2.11 & $\begin{array}{l}\text { Reaction time course and COPASI calculated time courses from } \\
\text { Scheme } 2.5 \mathrm{~B}\end{array}$ & 41 \\
\hline 2.12 & $\begin{array}{l}\text { Reaction time course and COPASI calculated time courses from } \\
\text { Scheme } 2.6 \mathrm{~A}\end{array}$ & 43 \\
\hline 2.13 & $\begin{array}{l}\text { Reaction time course and COPASI calculated time courses from } \\
\text { Scheme } 2.6 \mathrm{~A}\end{array}$ & 44 \\
\hline 2.14 & $\begin{array}{l}\text { Reaction time course and COPASI calculated time courses from } \\
\text { Scheme } 2.6 \mathrm{~B}\end{array}$ & 45 \\
\hline 2.15 & $\begin{array}{l}\text { Reaction time course and COPASI calculated time courses from } \\
\text { Scheme } 2.6 \mathrm{~B}\end{array}$ & 46 \\
\hline 2.16 & $\begin{array}{l}\text { Reaction time course and COPASI calculated time courses from } \\
\text { Scheme } 2.7\end{array}$ & 48 \\
\hline 2.17 & $\begin{array}{l}\text { Reaction time course and COPASI calculated time courses from } \\
\text { Scheme } 2.7\end{array}$ & 49 \\
\hline 2.18 & $\begin{array}{l}\text { Isomerization of linear Grignard to branched followed by } \\
\text { carboxylation, Area of Gaussian vs time. }\end{array}$ & 50 \\
\hline
\end{tabular}

-vii- 


\begin{tabular}{|l|l|l|}
\hline 2.19 & $\begin{array}{l}\text { Reaction time course and COPASI calculated time courses from } \\
\text { Scheme 2.7 }\end{array}$ & 52 \\
\hline 2.20 & $\begin{array}{l}\text { Reaction time course and COPASI calculated time courses from } \\
\text { Scheme 2.7 }\end{array}$ & 53 \\
\hline 2.21 & $\begin{array}{l}\text { Reaction time course and COPASI calculated time courses from } \\
\text { Scheme 2.7 (1.74 M Grignard) }\end{array}$ & 54 \\
\hline 2.22 & Kinetic predictions based on reactions and rates & 55 \\
\hline 2.23 & Thomas' experimental timecourse & 56 \\
\hline 2.24 & $\begin{array}{l}\text { Kinetic predictions based on reactions and rates tabulated in Table } \\
2.10 .\end{array}$ & 56 \\
\hline 3.1 & Frequency analysis of styrene derivatives & 61 \\
\hline 3.2 & Initial rates and observed rates Hammett Parameters & 61 \\
\hline 3.3 & Concentration dependencies of styrene derivatives & 64 \\
\hline 3.4 & COPASI calculated Reaction time courses & 65 \\
\hline 3.5 & Calculated natural charge of benzylic carbon relative to $\sigma$ & 67 \\
\hline 4.1 & Molecular structure of Thomas' intermediates & 70 \\
\hline 4.2 & Flask used for hydromagnesiation when a gas is generated & 71 \\
\hline 4.3 & Hydromagnesiation using Ethyl Grignard & 72 \\
\hline 4.4 & Hydromagnesiation timecourses with various Grignard reagents & 73 \\
\hline 4.5 & Concentration dependencies & 74 \\
\hline 4.6 & COPASI calculations for EthylMgBr & 77 \\
\hline 4.7 & COPASI calculations for IsopropylMgBr & 78 \\
\hline 4.8 & COPASI calculations low [styrene] and [Grignard] & 79 \\
\hline 5.1 & Time course and concentration dependencies of precatalysts & 82 \\
\hline 5.2 & COPASI calculations for (PDIMe)FeCl 2 & 86 \\
\hline 5.3 & COPASI calculations for (PDI-Me)FeCl2 & 87 \\
\hline & & \\
\hline
\end{tabular}


List of Schemes

\begin{tabular}{|l|l|l|}
\hline 1.1 & Iron CC catalysis described by Kochi & 5 \\
\hline 1.2 & Iron CC catalysis described by Fürstner & 6 \\
\hline 1.3 & Iron-NHC catalyzed CC reactions & 8 \\
\hline 1.4 & Transition Metal-catalyzed radical polymerization & 10 \\
\hline 1.5 & Transition Metal-catalyzed Ziegler-Natta polymerization & 11 \\
\hline 1.6 & Transition Metal-catalyzed hydrogenation & 13 \\
\hline 1.7 & Iron-catalyzed transfer hydrogenation & 15 \\
\hline 1.8 & Transition Metal-catalyzed hydrofunctionalization. & 16 \\
\hline 1.9 & Iron-catalyzed hydrosilylation & 18 \\
\hline 1.10 & Iron-catalyzed hydroformylation & 21 \\
\hline 1.11 & Iron-catalyzed hydrocarboxylation & 23 \\
\hline 2.1 & Grignard Utility & 27 \\
\hline 2.2 & Kochi iron-catalyzed transfer hydrometallation & 29 \\
\hline 2.3 & Thomas' Hydromagnesiation reaction conditions & 30 \\
\hline 2.4 & Iron-catalyzed hydromagnesiation with cyclopentylMgBr, GCYP. & 31 \\
\hline 2.5 & $\begin{array}{l}\text { Proposed mechanism introducing A) catalyst decomposition and B) } \\
\text { Grignard speciation. }\end{array}$ & 37 \\
\hline 2.6 & Proposed mechanism introducing a styrene catalyzed activation & 42 \\
\hline 2.7 & $\begin{array}{l}\text { Proposed mechanism introducing a catalyst decomposition pathway } \\
\text { by styrene }\end{array}$ & 47 \\
\hline 2.8 & Proposed mechanism of iron-catalyzed hydromagnesiation & 51 \\
\hline 3.1 & Thomas' Hydromagnesiation of styrene derivatives & 60 \\
\hline 3.2 & Possible explanation of negative $\rho$ value & 60 \\
\hline 3.3 & Proposed mechanism for styrene derivatives & 63 \\
\hline 4.1 & Thomas' Hydromagnesiation of Grignard variants & 70 \\
\hline 4.2 & Hydromagnesiation using various Grignards & 73 \\
\hline 4.3 & Proposed mechanism for Grignards generating gaseous olefins & 76 \\
\hline 5.1 & Effect of (PDI)iron-catalysts for polymerization & 80 \\
\hline 5.2 & Iron-catalyzed hydromagnesiation with various catalysts & 81 \\
\hline 5.3 & Proposed mechanism of iron-catalyzed hydromagnesiation & 84 \\
\hline 5.4 & $\begin{array}{l}\text { Proposed mechanism of iron-catalyzed hydromagnesiation } \\
\text { including off cycle catalyst cycle }\end{array}$ & 85 \\
\hline F.1 & Overall proposed mechanism of iron-catalyzed hydromagnesiation & 89 \\
\hline & & \\
\hline
\end{tabular}




\section{List of Abbreviations}

\begin{tabular}{|c|c|}
\hline acac & acetylacetonate \\
\hline $\mathrm{BHE} / \mathrm{MI}$ & $\beta$-hydride elimination/migratory insertion \\
\hline BHT & butylated hydroxytoluene \\
\hline BR & $\alpha$-arylethylmagnesium bromide \\
\hline $\mathrm{CC}$ & cross-coupling reactions \\
\hline COPASI & COmplex PAthway SImulator \\
\hline CYP & cyclopentene \\
\hline DCM & dichloromethane \\
\hline DHT & direct $\beta$-hydride transfer \\
\hline DiCOMP & diamond composite ReactIR probe \\
\hline DMA & $\mathrm{N}, \mathrm{N}$-dimethylacetamide \\
\hline G & Grignard \\
\hline $\mathrm{G}^{\text {cyp }}$ & cyclopentylmagnesium bromide \\
\hline $\mathrm{GEt}^{\mathrm{Et}}$ & ethylmagnesium bromide \\
\hline $\mathrm{G}^{\mathrm{iPr}}$ & isopropylmagnesium bromide \\
\hline $\mathrm{G}^{\mathrm{Pr}}$ & propylmagnesium bromide \\
\hline $\mathrm{G}^{\mathrm{cPr}}$ & cyclopropylmagnesium bromide \\
\hline $\mathrm{G}^{\mathrm{Bu}}$ & butylmagnesium bromide \\
\hline $\mathrm{G}^{\mathrm{iBu}}$ & isobutylmagnesium bromide \\
\hline $\mathrm{G}^{\mathrm{sBu}}$ & sec-butylmagnesium bromide \\
\hline $\mathrm{G}^{\mathrm{cBu}}$ & cyclobutylmagnesium bromide \\
\hline $\mathrm{G}^{\text {Pent }}$ & pentymagnesium bromide \\
\hline $\mathrm{G}^{\mathrm{Hex}}$ & hexylmagnesium bromide \\
\hline $\mathrm{G}^{\mathrm{cHex}}$ & cyclohexylmagnesium bromide \\
\hline IR & infrared \\
\hline $\mathrm{L}$ & $\beta$-arylethylmagnesium bromide \\
\hline LSODA & Livermore Solver for Ordinary Differential Equations \\
\hline MAO & methylaluminoxane \\
\hline $\mathrm{NHC}$ & $\mathrm{N}$-heterocyclic carbene \\
\hline NMR & nuclear magnetic resonance \\
\hline NSAIDs & Non-Steroidal Anti-Inflammatory Drugs \\
\hline PDI & 2,6-bis[1-(2,6-dialkylphenylimino)ethyl]pyridine \\
\hline PDI IPr & 2,6-bis[1-(2,6-diisopropylphenylimino)ethyl]pyridine \\
\hline $\mathrm{PDI}{ }^{\mathrm{Me}}$ & 2,6-bis[1-(2,6-dimethylphenylimino)ethyl]pyridine \\
\hline$\left.P D\right|^{2-M e}$ & 2,6-bis[1-(2-,methylphenylimino)ethyl]pyridine \\
\hline $\mathrm{PDI}{ }^{\mathrm{H}}$ & 2,6-bis[1-(phenylimino)ethyl]pyridine \\
\hline $\mathrm{S}$ & Styrene \\
\hline $\mathrm{S}^{\mathrm{Me}}$ & 4-methylstyrene \\
\hline SiBu & 4-isobutylstyrene \\
\hline $\mathrm{S}^{\mathrm{tBu}}$ & 4-tertbutylstyrene \\
\hline $\mathrm{S}^{\mathrm{OMe}}$ & 4-methoxystyrene \\
\hline THF & tetrahydrofuran \\
\hline TLS & Turnover Limiting Step \\
\hline TMEDA & N,N,N',N'-tetramethylethylenediamine \\
\hline
\end{tabular}




\section{Chapter 1: Introduction to Homogeneous Iron Catalysis}

\subsection{Historical Milestones}

The synthetic utility of iron in catalysis advanced in the latter half of the twentieth century with only a few momentous iron-catalyzed processes that were well known such as: Fenton Oxidation, Haber-Bosch, and Fischer-Tropsch methodologies (Figure 1.1). ${ }^{2}$ Haber-Bosch nitrogen fixation and Fischer-Tropsch hydrocarbon synthesis, though heterogeneous, result in a variety of caveats, namely the need for high pressure and temperatures for reactivity. The advancement of mimics for the Haber-Bosch nitrogen fixation and the Fischer-Tropsch processes has been a topic of interest for the scientific community recently. ${ }^{3}$ The Fischer-Tropsch synthesis of hydrocarbons from $\mathrm{CO}$ and $\mathrm{H}_{2}$ (syn-gas) has also been a topic of recent literature, but new developments primarily rely on heterogeneous catalysts or nanoparticles. However, Peters has also developed a homogeneous iron-centers for this process. ${ }^{4-8}$

One of the first homogeneous catalytic methodologies used for iron was Reppe's Carbonylation in $1953 .^{9,10}$ In the mid to late 1900 s, organometallic chemistry began to develop into a major branch of chemistry, namely with the development of Wilkinson's catalyst for olefin hydrogenation and the characterization of the iron porphyrin site in hemoglobin. Though at that time most catalyst development was reported with metals such as $\mathrm{Rh}, \mathrm{Pd}, \mathrm{Ru}, \mathrm{Pt}$, or Ti, beginning in 1971, iron catalysis began to gain momentum with the work of Kochi. ${ }^{11-13}$ In 1979, the expansion of iron porphyrin oxidation chemistry further advanced homogeneous iron catalysis not limited to enzymatic processes including cross-coupling (CC), polymerization, hydrogenations and hydro/hetero(element)functionalization. Figure 1.1 describes the timeline of iron catalysis. Additional iron catalyzed methodologies are discussed in the succeeding sections of this chapter. 

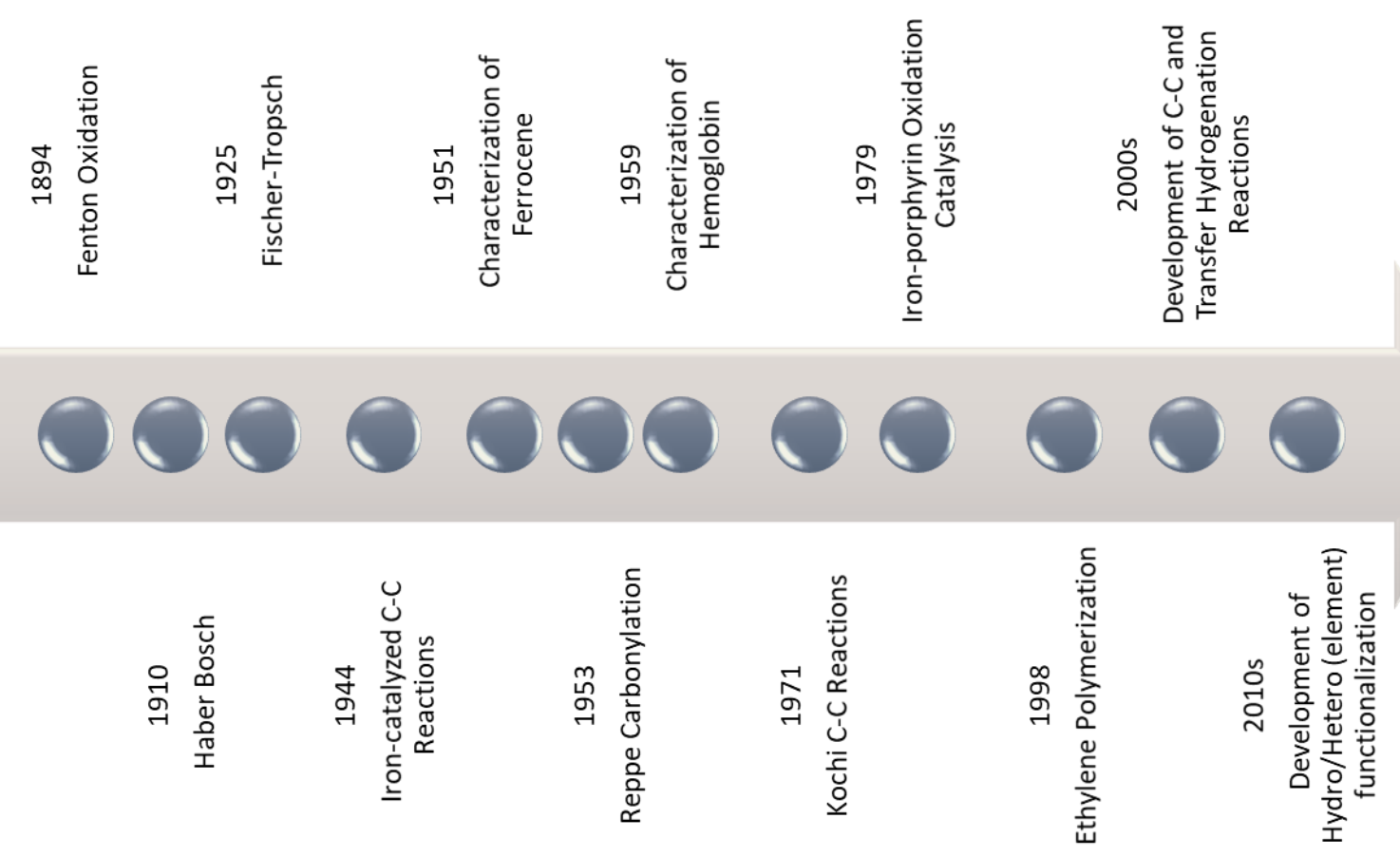

Figure 1.1: Iron catalysis timeline. ${ }^{2}$

\subsection{Iron and enzymes}

Beginning with the isolation and characterization of hemoglobin in 1959, there has been significant effort to understand enzymatic systems and active sites. ${ }^{2}$ The iron centered heme shown in Figure 1.2 is one of the most commonly mimicked for oxidation reactions. ${ }^{14}$ Traditionally, these heme iron complexes can activate dioxygen, generating a variety of superoxo, peroxo, oxo, and bridging superoxide iron species. ${ }^{14,15}$ Other common enzymes with iron-containing active sites are non-heme-ligated iron enzymes. Two common non-heme enzymes are amino acid-bound dioxygenases and the ironsulfur cluster based DNA-polymerase, shown in Figure 1.3. ${ }^{16-18}$ 
A

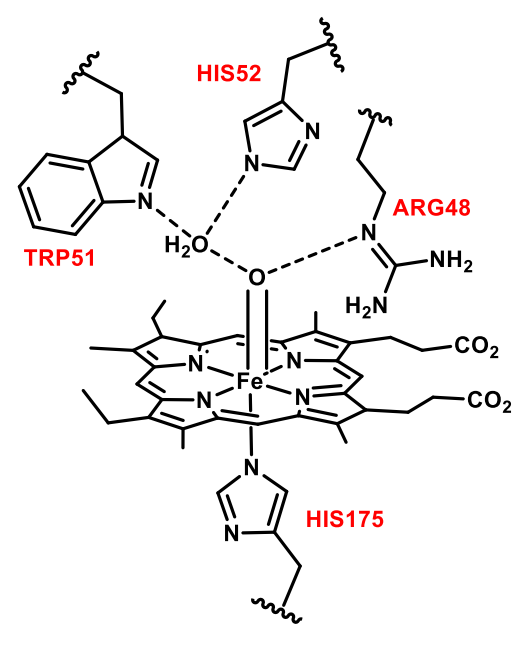

B

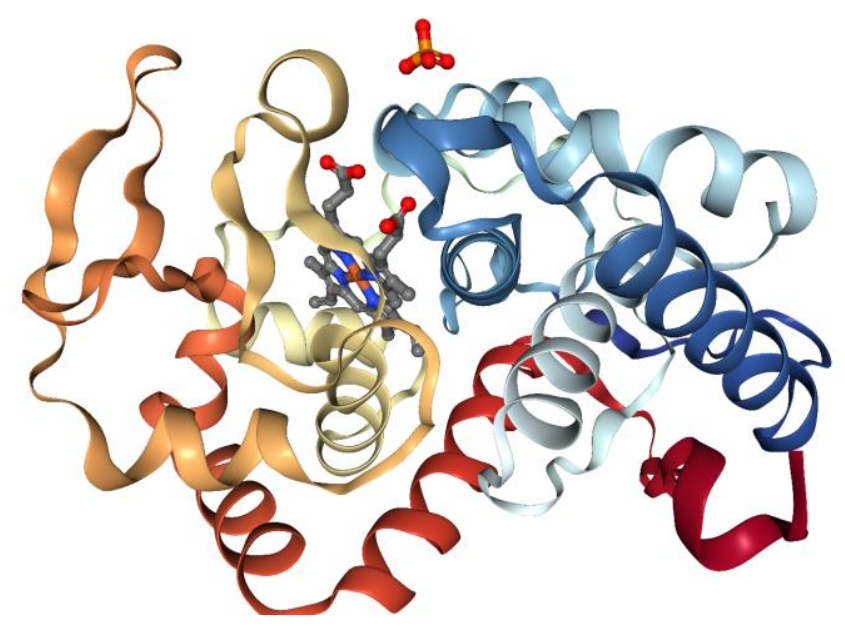

C

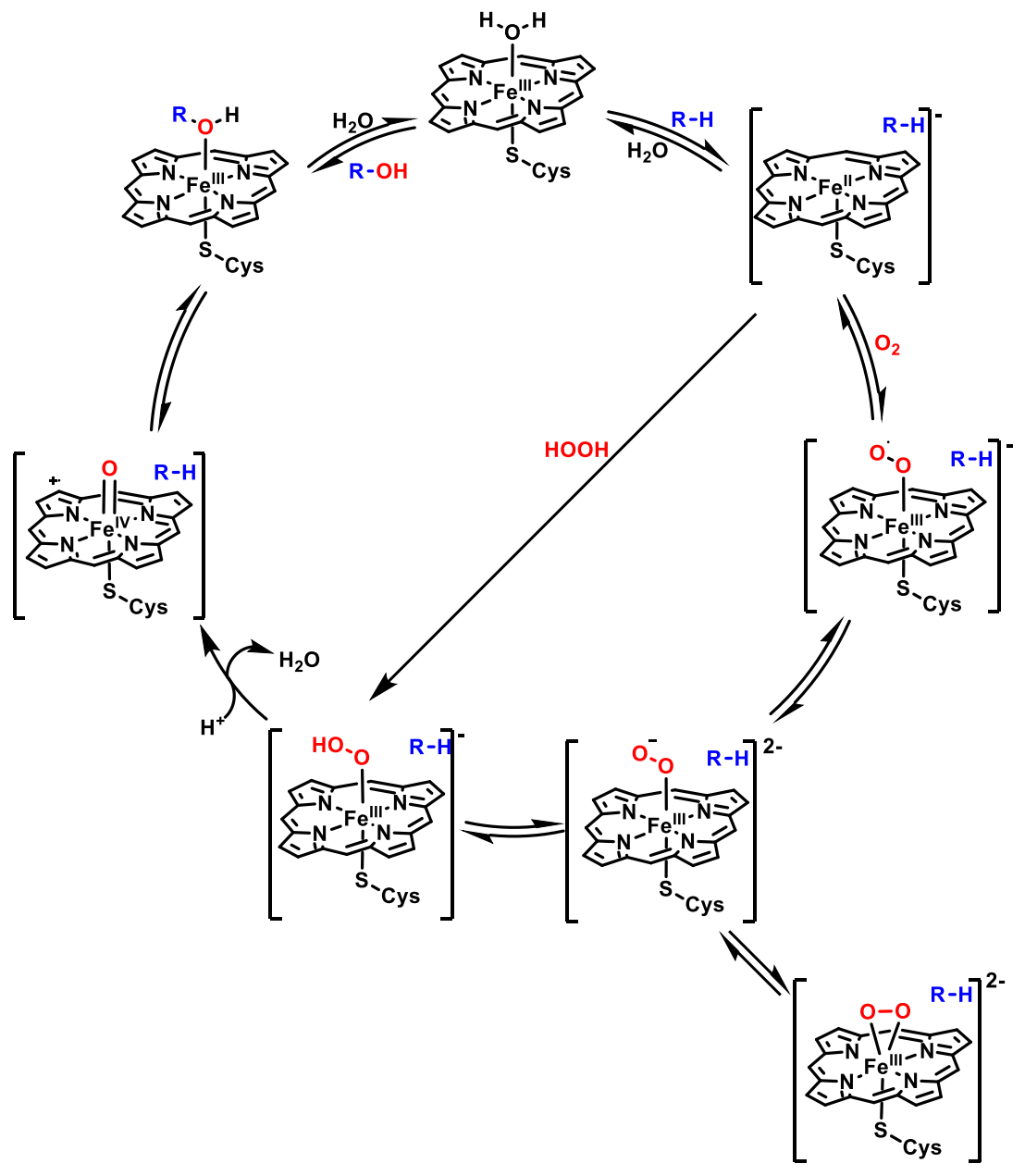

Figure 1.2: Iron heme enzyme catalysis A) active site of yeast cytochrome $c$ peroxidase ( $p d b=3 \mathrm{M} 23$ ); B) Protein structure of 3M23 from PDB; C) Enzymatic cycle of dioxygen activation. ${ }^{14-16}$ 
A

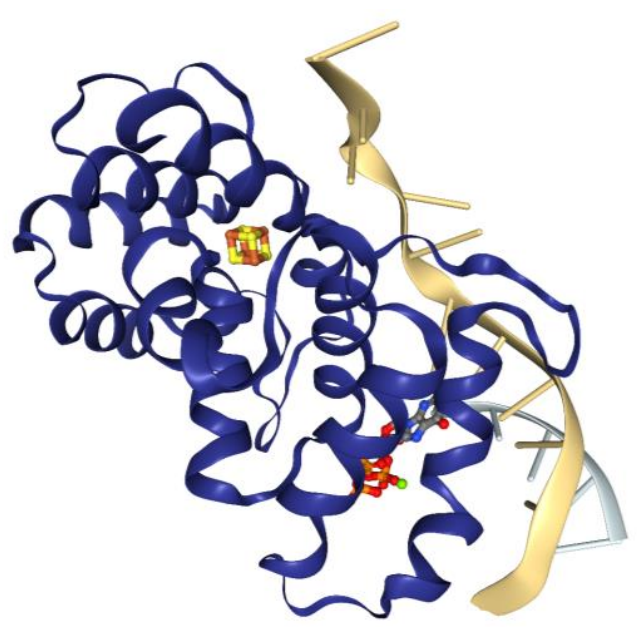

B

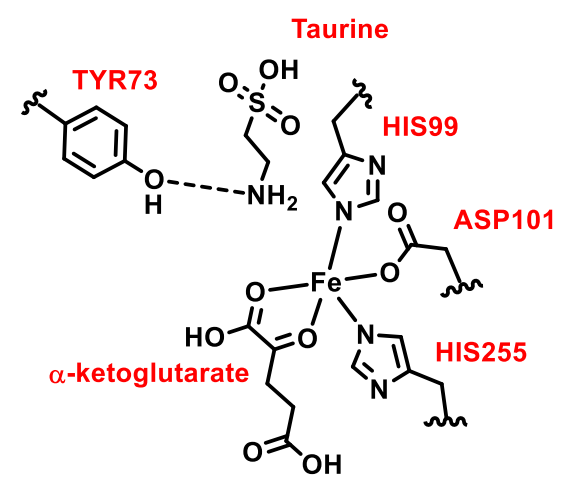

Figure 1.3: Iron non-heme enzymes $A$ ) iron-sulfur cluster DNA polymerase (PDB = $5 \mathrm{FOQ}$ ); B) Active site of taurine/a-ketoglutarate dioxygenase. ${ }^{16-18}$

\subsection{Iron-catalyzed cross-coupling}

Iron-catalyzed cross-coupling (CC) reactions fall under 3 main categories: Kumada-, Negishi-, and Suzuki-Miyaura-type reactions. The majority of which are the Kumada-type reactions. The remainder of this section will discuss Kumada-type CC reactions. This category of $\mathrm{CC}$ reactions is separated into two subclasses (simple iron-salt and ligated iron-catalyzed CC reactions). In 1944, Vevon and Mottez developed the first ironcatalyzed CC reactions, utilizing a simple (iron(III) chloride) to catalyze the coupling of aryl Grignards with alkyl bromides in moderate yields. ${ }^{19}$ Nearly thirty years passed until Kochi and coworkers identified the synthetic utility and began mechanistic studies of ironcatalyzed CC reactions. ${ }^{11,12}$ In 1976, Kochi's mechanistic studies identified limitations to iron-catalyzed CC reactions: 1) stereoselective homocoupling; 2) exchange and disproportionation; and 3) structural effects of the Grignard reagent. ${ }^{12}$ Traditionally, the mechanism for iron-catalyzed $\mathrm{CC}$ reactions mimic nickel and palladium catalyzed Kumada couplings (Scheme 1.1).20-23 The reaction starts with an oxidative addition of an alkyl or alkenyl bromide to iron(I), followed by transmetallation with a Grignard reagent and reductive elimination. The shortcomings of stereoselective homocoupling arise from transmetallation of the iron(III) vinyl resulting in a vinylic Grignard. This vinylic Grignard reagent undergoes transmetallation with a (vinyl)iron(III) bromide to generate a dialkenyl 
iron(III) that reductively eliminates to generate a homocoupled product such as hexadiene as illustrated in Scheme 1.1.
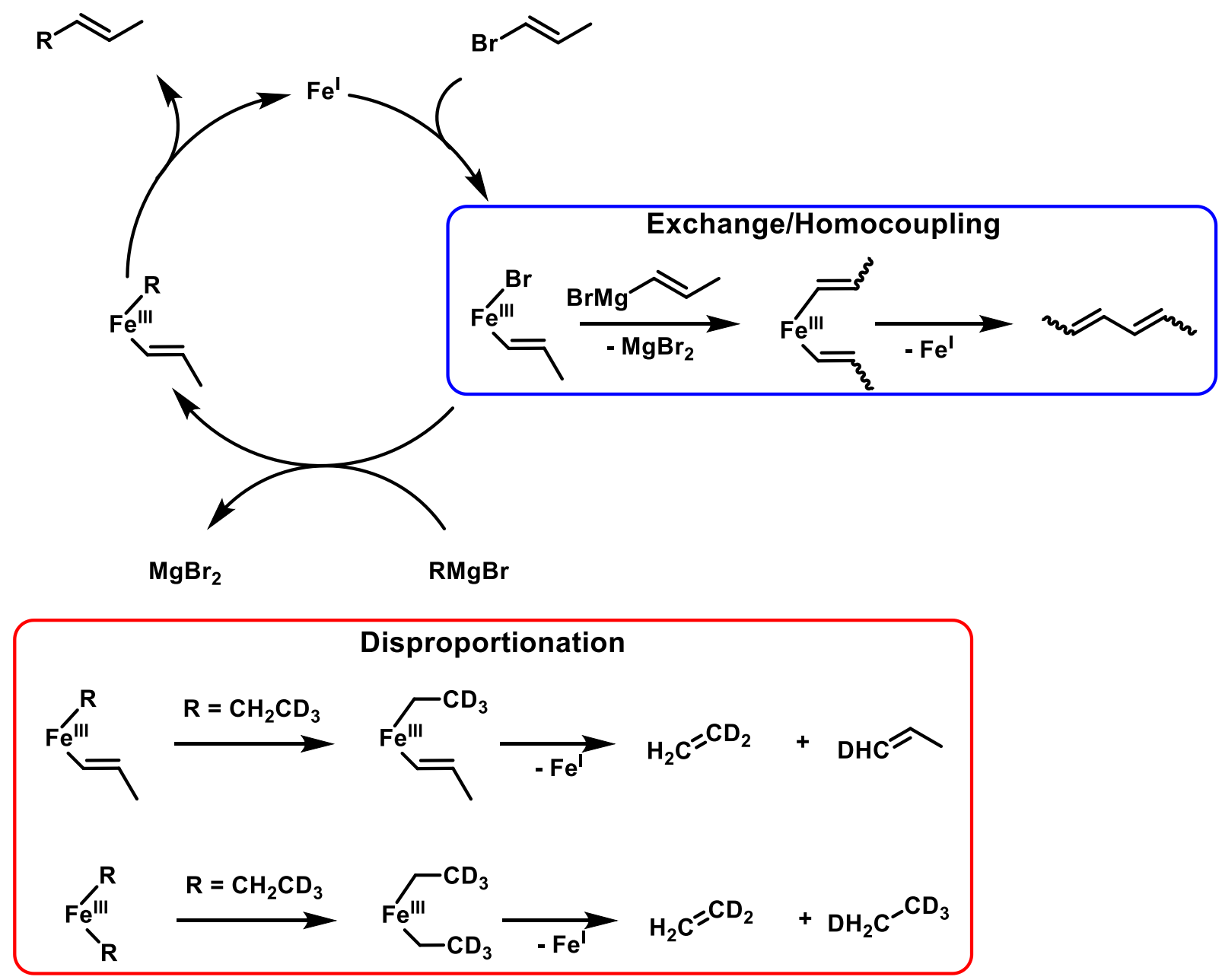

Scheme 1.1: Iron CC catalysis described by Kochi. ${ }^{*}$ Reproduced in ChemDraw ${ }^{12,13}$

Until contributions from Cahiez and Knochel using simple iron salts, in 1998, the field of iron-catalyzed CC reactions remained relatively less attractive in comparison to the widely utilized $\mathrm{Ni}$ and $\mathrm{Pd}$-catalyzed $\mathrm{CC}$ reactions, shown in Figure 1.4 (top). ${ }^{24}$ Ironcatalyzed $\mathrm{CC}$ reactions have gained interest after the development of inorganic Grignard reagents and the similarity of these reagents to intermediates of proposed CC pathways. ${ }^{25}$ The use of additives, $N$-methylpyrrolidone, as cosolvents was determined to aid in the stabilization of the active inorganic Grignard reagent without binding directly to the iron center and prevents the formation of iron-alkyl clusters. ${ }^{26}$ In the early 2000 s, 
Fürstner and Nakamura extended the scope of iron-catalyzed CC reactions with iron(III) precatalysts in the presence of coordinating ligands. ${ }^{27,28}$ Fürstner proposed an iron-ate catalyzed CC, in which an inorganic Grignard was the active iron species (Scheme 1.2).

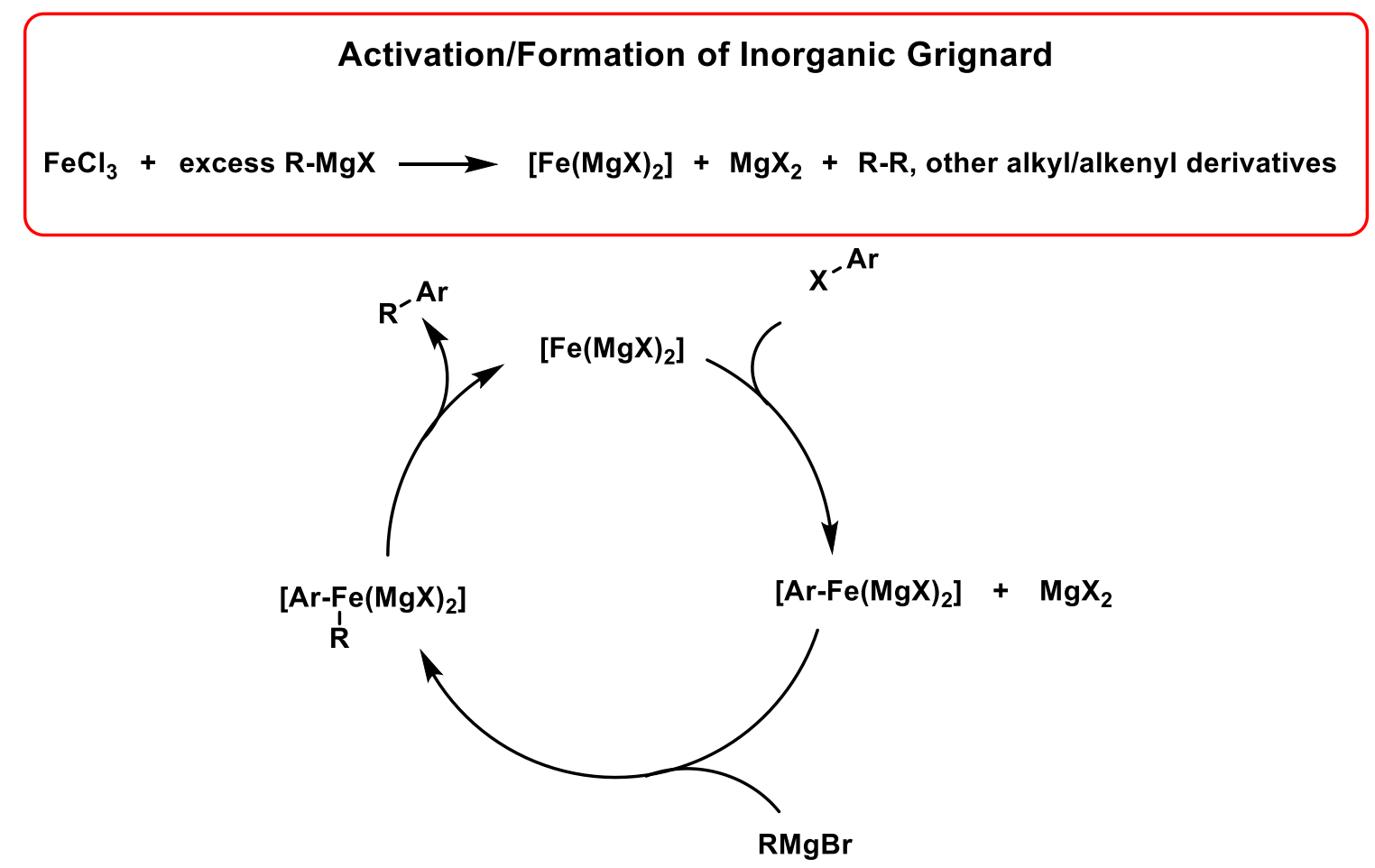

Scheme 1.2: Iron CC catalysis described by Fürstner. *Reproduced using ChemDraw. ${ }^{27}$

Since the work of Nakamura and Fürstner, there have been strides in the mechanistic understanding of iron-catalyzed CC reactions. The development of newer catalyst-ligand systems has aided in issues with reactivity. Figure 1.4 describes a variety of ironcatalyzed CC reactions. Other common ligand motifs for CC reactions include amines and phosphines (not shown). More expansive classes of ligands such as N-Heterocyclic carbenes (NHCs) have been utilized more recently. 
Negishi-type

$\mathrm{R}_{2 \mathrm{Br}}+\mathrm{Zn}\left(\mathrm{I}^{\mathrm{R}}\right)_{2} \stackrel{\text { Fe cat. }}{\longrightarrow} \mathrm{R}_{\mathrm{R}} \sim \mathrm{R}^{\prime}$

Nakamura et al Org. Lett. 2009

Suzuki Miyaura

$\stackrel{\mathrm{O}^{\prime} \mathrm{B}_{\mathrm{O}} \mathrm{O} \chi}{\mathrm{Ar}^{\prime}}+\mathrm{R}^{-X} \stackrel{\text { Fe cat. }}{\longrightarrow} \mathrm{Ar}-\mathrm{R}$

Nakamura et al JACS 2010
Cross-Coupling in Pharmaceuticals<smiles>CN1CCCC1c1cccnc1</smiles>

(S)-nicotine<smiles>CN1CCC(COCc2cc(C(F)(F)F)cc(C3(c4ccc(F)cc4)CC3)n2)(c2ccc(F)cc2)CC1</smiles>

Antidepresent<smiles>Cc1ccc(C2O[C@H](CO)C[C@@H](O)[C@H]2O)cc1Cc1ccc(-c2ccc(F)cc2)s1</smiles><smiles>CCc1c(-c2ccc(C(C)(C)O)cc2)[nH]c2nccnc12</smiles>

Anti-cancer target

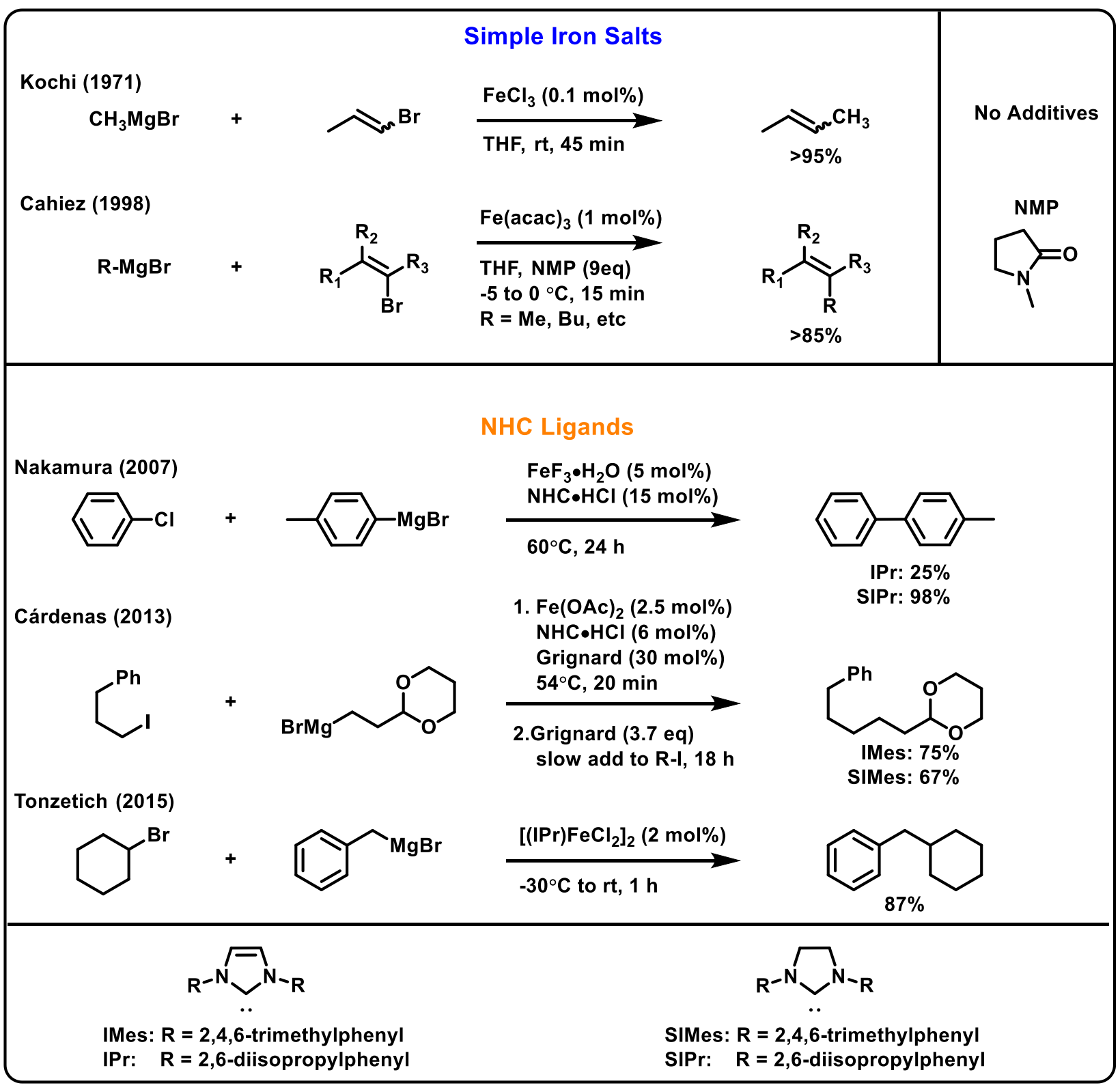

Figure 1.4: Common iron-catalyzed CC reactions reproduced from a Chemical Review. ${ }^{24,27-51}$ 


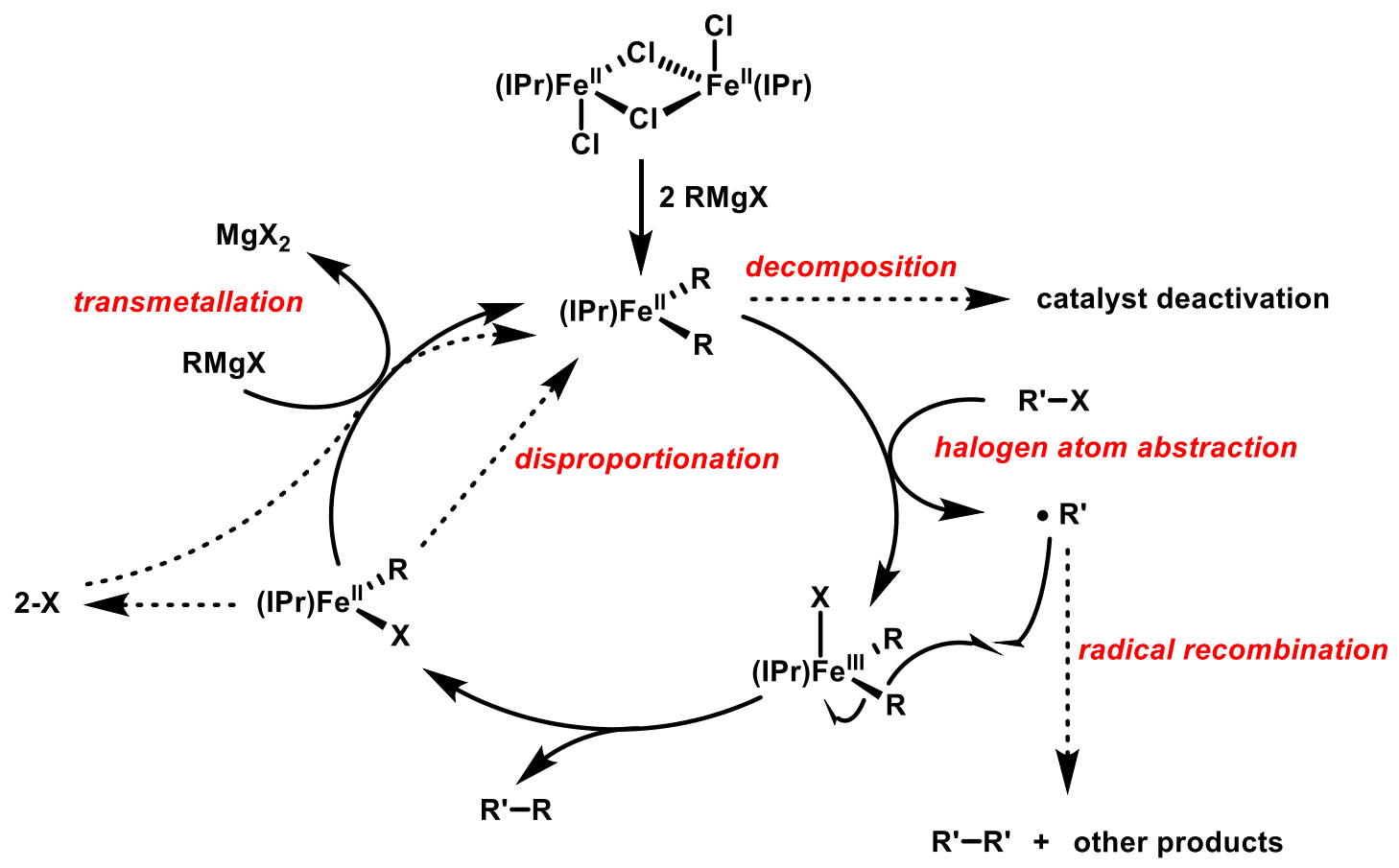

Scheme 1.3: Reproduced mechanism of iron-NHC catalyzed CC reactions. ${ }^{43}$

Beginning in 2007, N-heterocyclic carbene (NHC) ligands were introduced in ironcatalyzed CC reactions. ${ }^{43}$ Through recent mechanistic studies, Tonzetich found that the iron-catalyzed CC reaction mechanism which was predicted to be a traditional oxidative addition, transmetallation, reductive elimination path could be expanded upon. In the case of iron-NHCs, a radical-based iron(II/III) cycle is more favorable, thus avoiding the use of less common oxidation states for iron. The reactivity of iron-NHCs for $\mathrm{CC}$ reactions of Grignards with alkyl halides as electrophiles has commonly introduced electrophilic radical recombination. The radical-based mechanism is substantiated upon reaction inhibition by butylated hydroxytoluene, $\mathrm{BHT} .{ }^{43}$ Scheme 1.3 describes the mechanism of iron-NHC catalyzed CC reactions proposed by Tonzetich. Further isolation and characterization of reaction intermediates will enhance the definition of the two subclasses and continue the ongoing advancement of iron-catalyzed CC chemistry. 


\subsection{Iron-catalyzed polymerization}

Transition metal-catalyzed polymerization has been investigated since Ziegler and Natta won the Nobel Prize over 50 years ago. There are two classes of polymerization reactions with transition metals: 1) traditional Ziegler-Natta catalysis and 2) radical-based methodologies. Polymerization of alkenes to yield a-polyolefins using either ethylene or propylene began in the mid-1900s with the work of Ziegler and Natta. ${ }^{52}$ Their development in ethylene polymerization has been elaborated upon by multiple research labs. ${ }^{53-79}$ Prior to 1998 , transition metals used in this type of catalysis were limited to $\mathrm{Ti}, \mathrm{Zr}, \mathrm{Ni}$, and $\mathrm{Pd}$. ${ }^{53-}$ 59 In 1998, Brookhart developed a homogeneous iron catalyst bearing a tridentate pyridine diimine (PDI) ligand, which has been the main ligand framework for iron- and other transition metal-catalyzed ethylene polymerization, Figure 1.5 .60

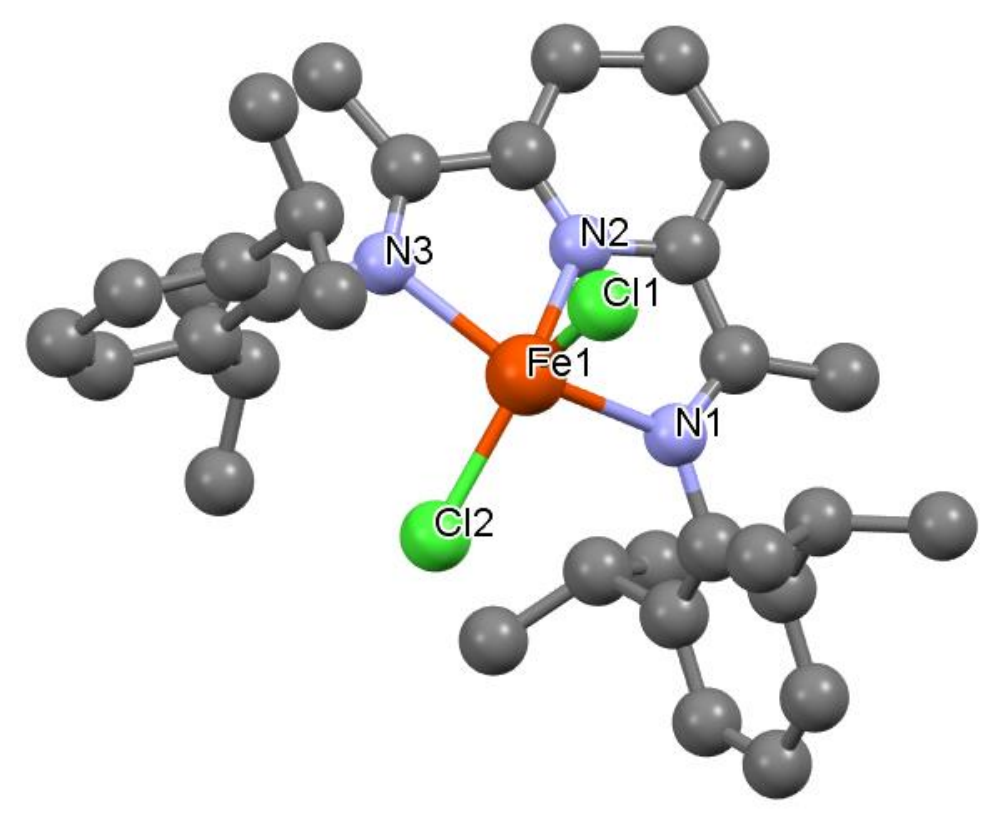

Figure 1.5: X-ray molecular structure structure of $(\mathrm{PDI} \mid \mathrm{Pr}) \mathrm{FeCl}_{2 .}{ }^{*}$ Reproduced from CSD Entry: PUGWIX.60 


\section{Activation}
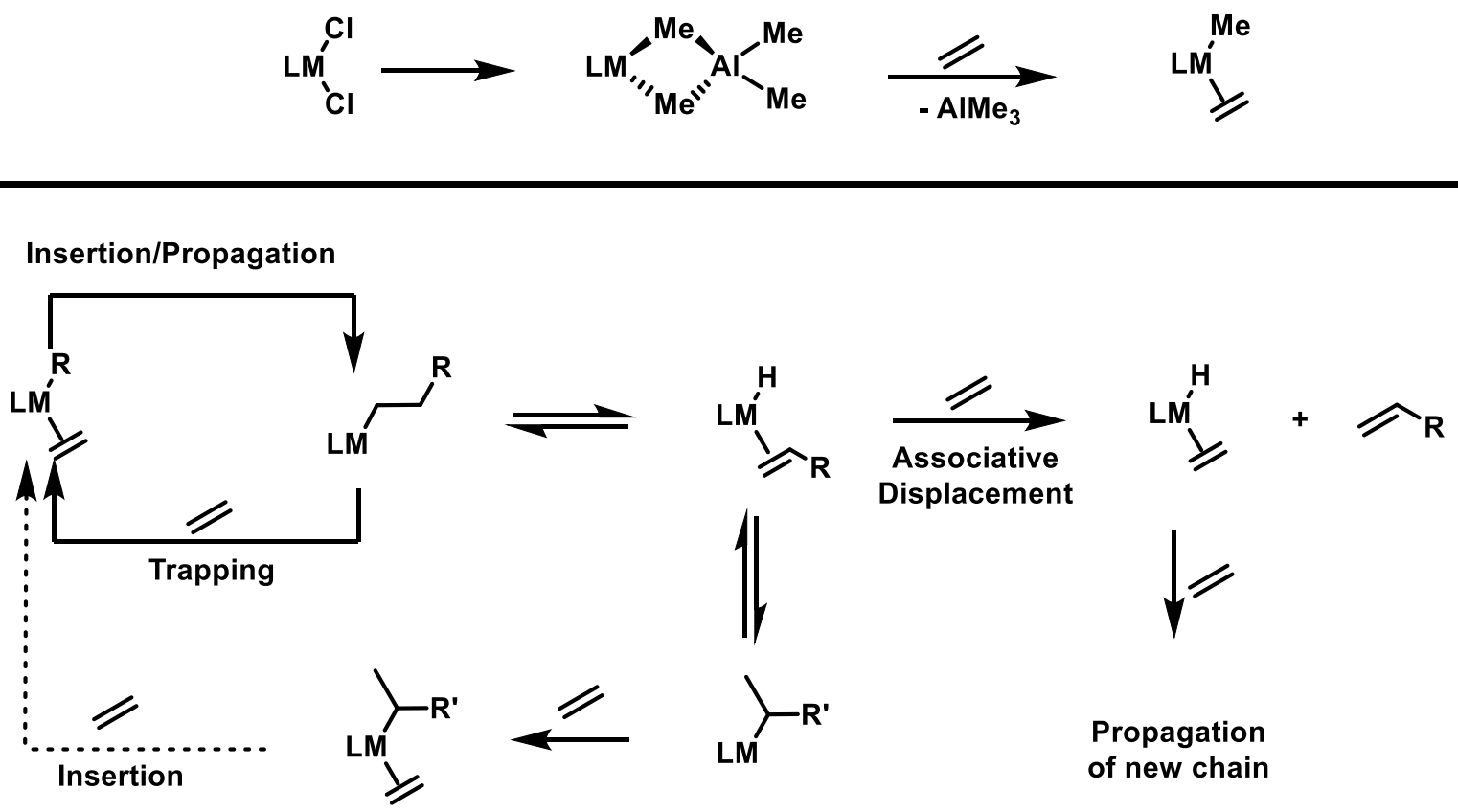

Scheme 1.4: Reproduced Transition metal-catalyzed Ziegler-Natta polymerization. 53-79

The mechanistic developments into Ziegler-Natta polymerization have proven to be quite difficult and complex. Reaction intermediates derived from the reactivity of the cocatalyst methylaluminoxane, MAO, with the iron center have driven the need for kinetic/mechanistic advancement. The work of Brookhart, ${ }^{60}$ Britovsek, ${ }^{61,62,65,66}$ Talsi, ${ }^{64,68,69,76,77}$ and Chirik ${ }^{70,78}$ have significantly advanced this field. The reaction mechanism of chain growth polymerization has undergone multiple analyses, most of which agree on the mechanism of catalysis in Scheme 1.4. From the beginning of the polymerization iron-age to the reactivity of other base metal catalysts, transition metal catalyzed polymerization has evolving theories in understanding owing to the work of multiple research labs.

The second class of polymerization, a more contemporary radical based polymerization began in the 1990s. ${ }^{80-85}$ Traditionally, $\mathrm{Cu}, \mathrm{Ni}, \mathrm{Pd}, \mathrm{Rh}$, and $\mathrm{Ru}$ were used for catalysis. In these catalytic systems, the processes of atom transfer radical addition (ATRA) or atom transfer radical polymerization (ATRP) are primarily the two main categories of polymerization. Mechanistically, these reactions undergo a transition metal 
controlled radical polymerization. This reaction is typical of styrenes and methacrylates initiated by an alkyl halide such as 1-phenylethyl chloride. ${ }^{80-85}$ Scheme 1.5 describes the difference between ATRA and ATRP. ATRP reactions were kinetically studied with copper catalysts. Matyjaszewski and collaborators led the field of homogeneous ATRP reaction mechanistic developments. They found that ATRP exhibits first order kinetics with respect to initiator, catalyst, and monomer concentration. Polymerization was determined to not be a simple inverse first order with respect to initial metal halide concentration, owing to the "persistent radical effect". ${ }^{84}$ This radical effect is attributed to the regulation of polymerization by guaranteeing that the rate of propagation and subsequent polymerization is significantly faster than radical recombination followed by disproportionation. ${ }^{84}$

\section{Atom Transfer Radical Addition, ATRA}

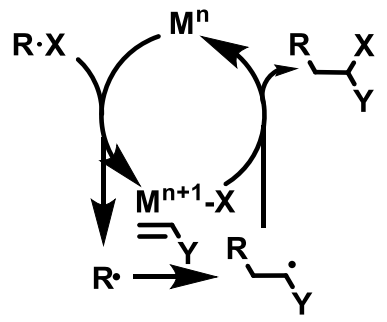

Atom Transfer Radical Polymerization, ATRP

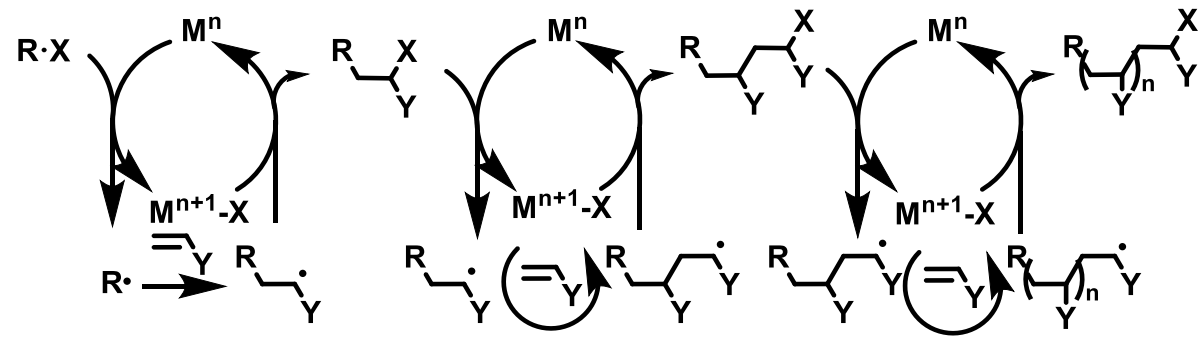

Scheme 1.5: Transition metal-catalyzed radical polymerization. *Reproduced in ChemDraw. ${ }^{53,80-85}$

\subsection{Iron-catalyzed hydrogenation}

Transition metal-catalyzed hydrogenation has been known since the late 1800s, owing to the work of Paul Sabatier, the father of hydrogenation and 1912 Nobel Prize winner in chemistry. ${ }^{86}$ Sabatier's process of hydrogenation has now expanded into multiple fields, such as production of fuel and other manufacturing processes in the soap industries. ${ }^{86}$ The Haber-Bosch and Fischer-Tropsch processes were the first hydrogenations using iron catalysis, see section 1.1 for details. ${ }^{2}$ Traditionally homogeneous hydrogenation used 
precious metals seen in the work of Wilkinson $(\mathrm{Rh})^{87}$ and others $\left(\mathrm{Pd}\right.$, Ir, etc). ${ }^{88,89}$ The hydrogenations using dihydrogen $\left(\mathrm{H}_{2}\right)$ as the hydride source all have two common reaction mechanisms, consisting of either a monohydride or a dihydride intermediate. $\mathrm{A}$ mechanism containing a monohydride intermediate is a combination of five elementary steps: 1) generation of a metal hydride, 2) coordination of an alkene, 3) migratory insertion, 4) coordination of dihydrogen, and 5) heterolytic cleavage of the $\mathrm{H}-\mathrm{H}$ bond. ${ }^{88,89}$ A dihydride intermediate mechanism also consists of five elementary steps: 1) dissociation of a ligand, 2) oxidative addition to generate a metal-dihydride, 3) coordination of alkene, 4) insertion into one metal-hydride, and 5) reductive elimination, Scheme 1.6.88,89 In the case of monohydride-based mechanisms, carbonyls are preferred over terminal alkenes/alkynes which are preferred over internal alkenes/alkynes. The dihydride intermediate pathway is proposed for catalysis involving $d^{8} \operatorname{Rh}(I)$ and $\operatorname{Ir}(I) .{ }^{89}$ Further development into this field with base metal catalysts such as $\mathrm{Fe}, \mathrm{Co}$, and $\mathrm{Mn}$ instead of noble metals has renewed interest in the comparison of hydrogenation reactions. ${ }^{89-110}$

One of the first instances of homogeneous iron-catalyzed hydrogenation was in the early 1960s where Frankel, Emken, and Davison from the Northern Regional Research Laboratory in Illinois published the hydrogenation of methyl linolenate by iron pentacarbonyl complexes. ${ }^{90}$ In their studies, they found that the hydrogenation of trienes primarily generated mono-enes with low yields of a diene. The diene was commonly conjugated and complexed with iron to generate a (diene)iron(III)-carbonyl complex. These initial studies led the way for Miller and Grant to determine the reaction kinetics of gas-phase hydrogenation of ethylene by $\mathrm{Fe}(\mathrm{CO})_{3}\left(\mathrm{C}_{2} \mathrm{H}_{4}\right)_{2} \cdot{ }^{93}$ Miller and Grant's studies used UV laser irradiation of ethylene, hydrogen, and the catalyst, yielding only ethane. Mechanistic developments in solvent-phase iron-catalyzed hydrogenation reactions resulted in a better understanding of catalysis by base metals. ${ }^{94} \mathrm{~A}$ variety of iron catalysts bearing redox non-innocent ligands have been utilized in hydrogenation of alkenes/alkynes and polar unsaturated bonds are shown in Figure 1.6. The use of noninnocent ligands developed by Chirik are of interest due to the ability of the ligand to stabilize a reduced iron center. Similar to Wilkinson's hydrogenation catalysis with $\mathrm{Rh}(\mathrm{I})$, 
Chirik's iron-catalyzed hydrogenation consists of a stepwise mechanism for hydride insertion (Scheme 1.6). ${ }^{95}$

Monohydride Intermediates

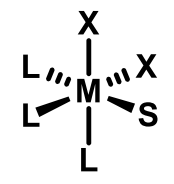

$\downarrow \begin{aligned} & +\mathrm{H}_{2} \\ & \downarrow-\mathrm{HX}\end{aligned}$

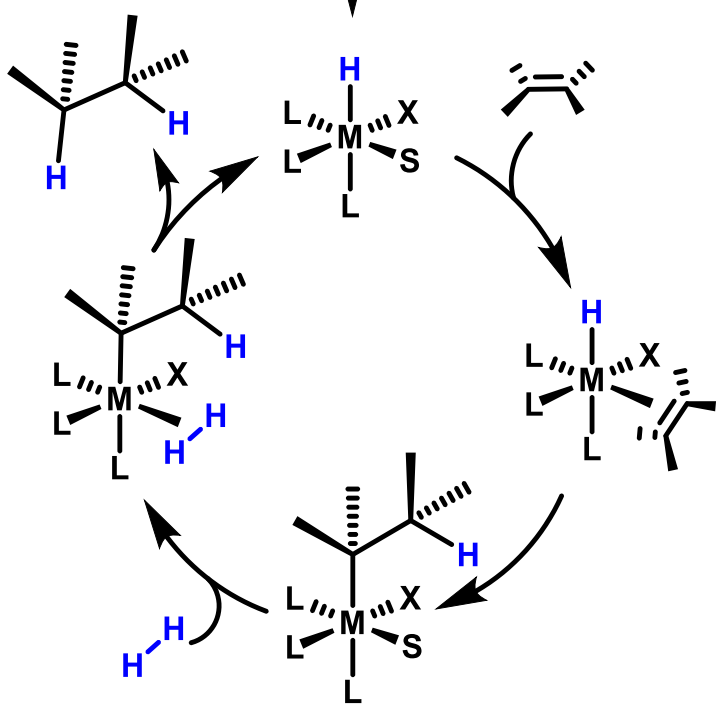

Dihydride Intermediates

$$
L^{L \prime \prime} M{ }^{\prime \prime X}
$$

$\sqrt{-L}+S$

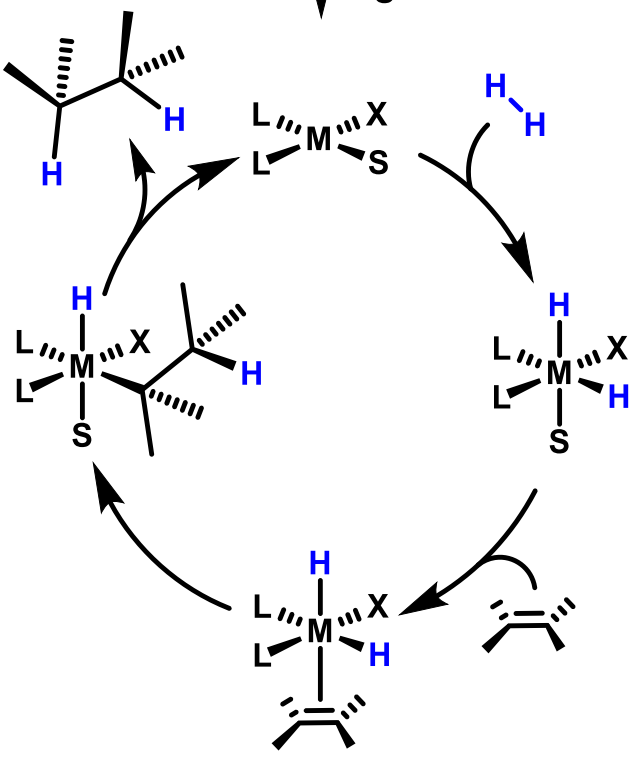

$M=R u, R h, I r$, Os, etc.

$\mathbf{L}=$ Ligand $\left(\mathbf{P R}_{\mathbf{3}}\right.$ and others)

$\mathbf{S}=$ solvent (ether, THF, methanol)

Scheme 1.6: Reproduced Transition metal-catalyzed hydrogenation. 88,89 


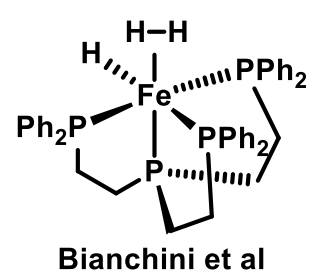

Organometallics 1992

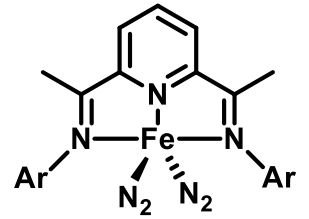

Bart and Chirik et al JACS 2004

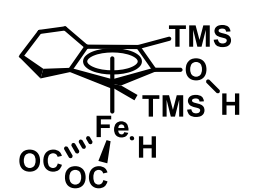

Casey and Guan JACS 2007

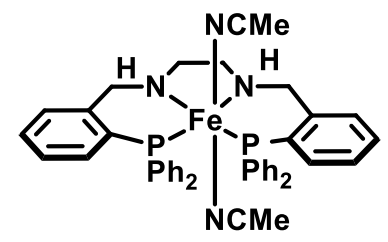

Morris et al ACIE 2008

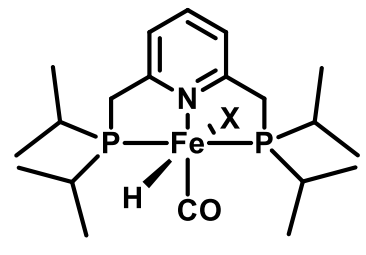

Milstein et al Chem. Eu. J. 2012

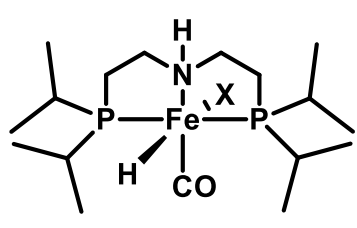

Jones et al ACS Catal. 2016

Figure 1.6: Iron-catalyzed hydrogenation catalysts. ${ }^{94,95,98,100,102,108}$

A third type of hydrogenation is transfer hydrogenation, more common in polar $\mathrm{C}=\mathrm{R}$ bonds $(R=C, O, N)$ substrates. Transfer hydrogenation uses ligand cooperativity to aid in hydride transfer. This process typically occurs through an outer sphere interaction, and the hydrogen source is not necessarily $\mathrm{H}_{2}$ but may be an alcohol or amine. The iron analog of Shvo's catalyst, by Casey and Guam, are commonly used transfer hydrogenation catalysts. ${ }^{89,98,108}$ The mechanism for transfer hydrogenation begins with the heterolytic cleavage of $\mathrm{H}_{2}$ or transfer of a hydride and proton from an alcohol (isopropanol) generating a metal-hydride and a protonated ligand. The coordinated polarized $\mathrm{C}=\mathrm{C}$ undergoes insertion into the metal-hydride, then subsequently the reduced C-C moiety is generated by transfer of the hydrogen from the ligand. The protonated ligand and metal hydride are then regenerated by either reactivity of an alcohol or heterolytic cleavage of $\mathrm{H}_{2}$ through a six-membered transition state (Scheme 1.7). ${ }^{108}$ Although many groups have studied hydrogenation reactions mechanistically, there are still developments that can arise from this methodology in the future. 
A

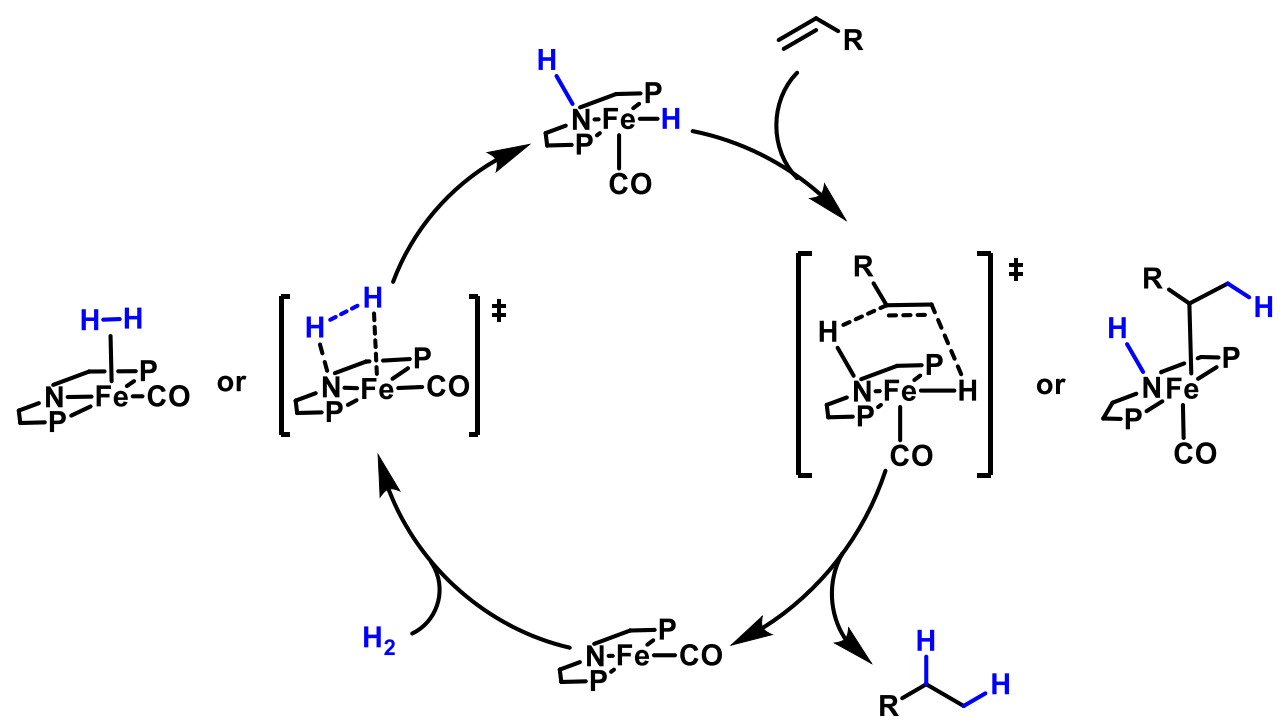

B

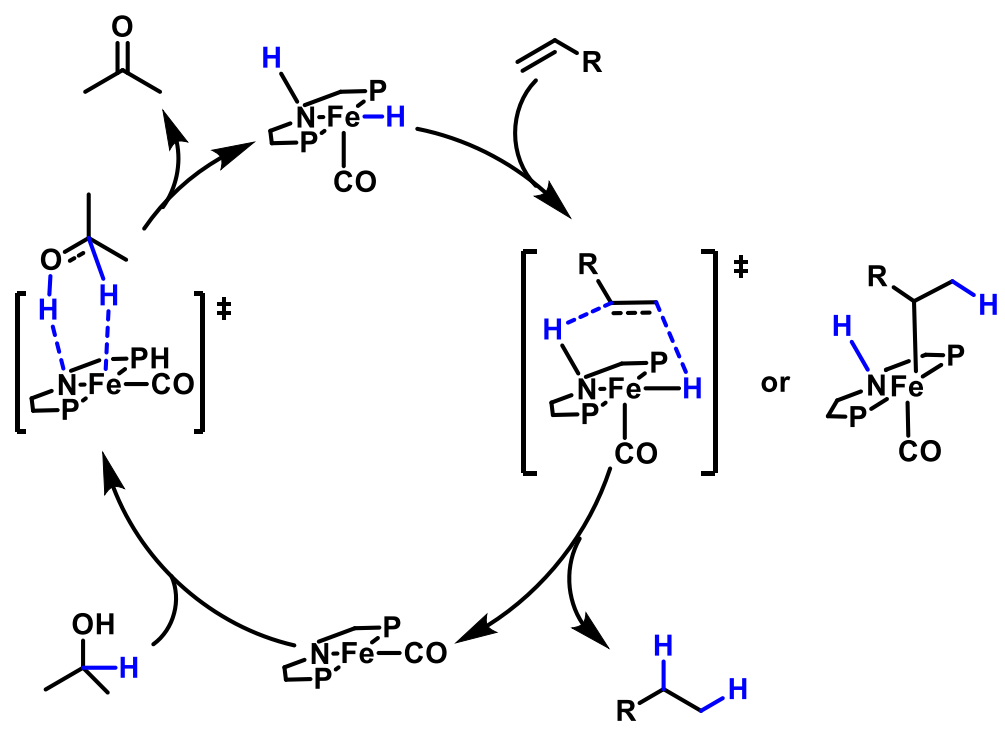

Scheme 1.7: Reproduced Iron-catalyzed transfer hydrogenation of alkenes. A) with dihydrogen; B) alcohol as hydride source.

\subsection{Iron-catalyzed hydrofunctionalization}

Hydrofunctionalization of unsaturated bonds prior to the early 2000s was catalyzed by noble-metals such as $\mathrm{Pd}, \mathrm{Rh}, \mathrm{Ir}, \mathrm{Ru}$, and Au. However, recent advancements beginning in 2010 allowed for the use of $\mathrm{Fe}, \mathrm{Co}, \mathrm{Mo}, \mathrm{Cu}$, and Ni catalysts. Hydrosilylation, 95,104,111124 hydroboration, ${ }^{116-118,125-138}$ hydroamination, ${ }^{139-142}$ hydroformylation, ${ }^{143-149}$ hydrocarboxylation, ${ }^{150-163}$ and hetero(element)functionalization ${ }^{164-167}$ are common classes of hydro/hetero(element)functionalization reactions. The processes of hydrosilylation, hydroboration, hydroformylation, and hydrocarboxylation are summarized below. Each 
class of hydro/hetero-functionalization reactions follow a similar mechanism as seen in Scheme 1.8. Traditionally, Scheme 1.8 B is favored for the reaction mechanism in which the cycle consists of 4 main elementary steps: oxidative addition, alkene coordination, migratory insertion, and reductive elimination.

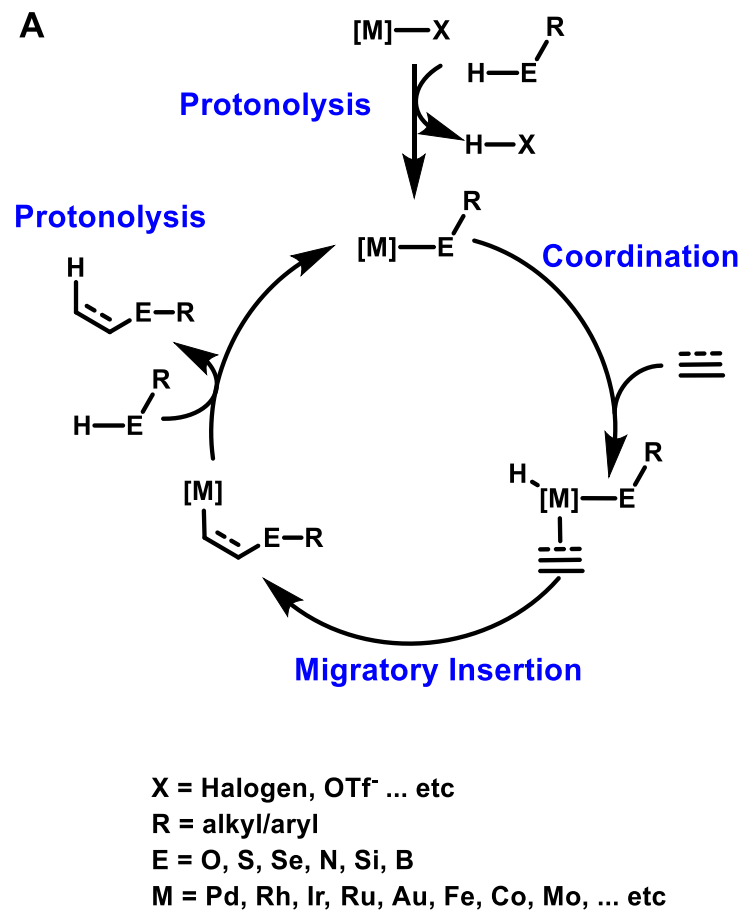

B

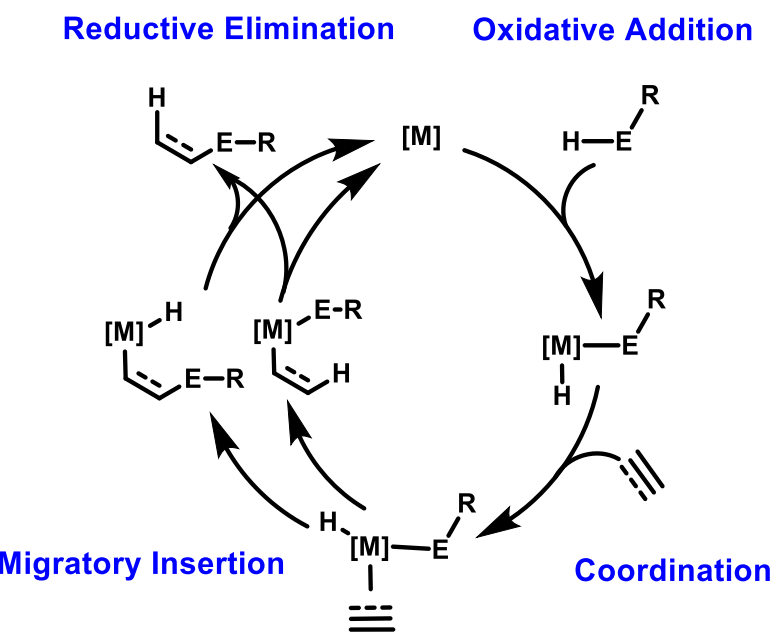

Scheme 1.8: Transition metal-catalyzed hydrofunctionalization. A) protonolysis based functionalization and B) traditional oxidative addition then reductive elimination functionalization. *Reproduced using ChemDraw

In 1973, Karstedt was able to initially catalyze a hydrosilylation of an unsaturated bond using Pt and other noble metals. ${ }^{168,169}$ Karstedt's platinum catalyst [Pt2(vinylsiloxane) $\left.{ }_{3}\right]$ improved selectivity and solubility of polysiloxane composites, which enabled better manufacturing routes of multiple commodities, such as oils, adhesives, injection molding products, etc. ${ }^{169}$ Further mechanistic studies in noble metal-catalyzed hydrosilylation reactions of olefins introduced common side reactions such as dehydrogenative silylation, hydrogenation, olefin isomerization, polymerization, and redistribution of hydrosilanes as (Figure 1.7). ${ }^{169}$ Prior to the groundbreaking work of Chirik and Bart, iron and other base metal systems often favored undesired side reactions and required harsh reaction $-16-$ 
conditions or photosynthetic measures to generate the active catalyst. ${ }^{169}$ Chirik and Nakajima discuss the difficulty of controlling iron catalysts due to numerous possible spin states at iron. ${ }^{95,111,113-116,120}$ Development of a well-defined ligand framework introduced a means of favoring hydrosilylation over the side reactions and in some cases increased 1,2- vs 2,1- insertion and $\alpha$ - (Markovnikov substitution) vs. $\beta$ - (Anti-Markovnikov substitution) regioselectivity (Figure 1.8). ${ }^{95,111,113-116,120}$ Recently, regioselectivity of hydrosilylation has been determined for both aliphatic and aromatic olefins. For reagents containing aromatic olefins, a-silylation (Markovnikov substitution, Scheme 1.9A) is favored; whereas, aliphatic olefins favor $\beta$-silylation (Anti-Markovnikov substitution, Scheme 1.9B). ${ }^{95,111,113-116,120,170}$ Based upon known limitations of iron-catalyzed hydrosilylation, future methodology development is a necessity to improve regioselectivity, reduce side product pathways, and provide catalyst control by using redox-active ligands.

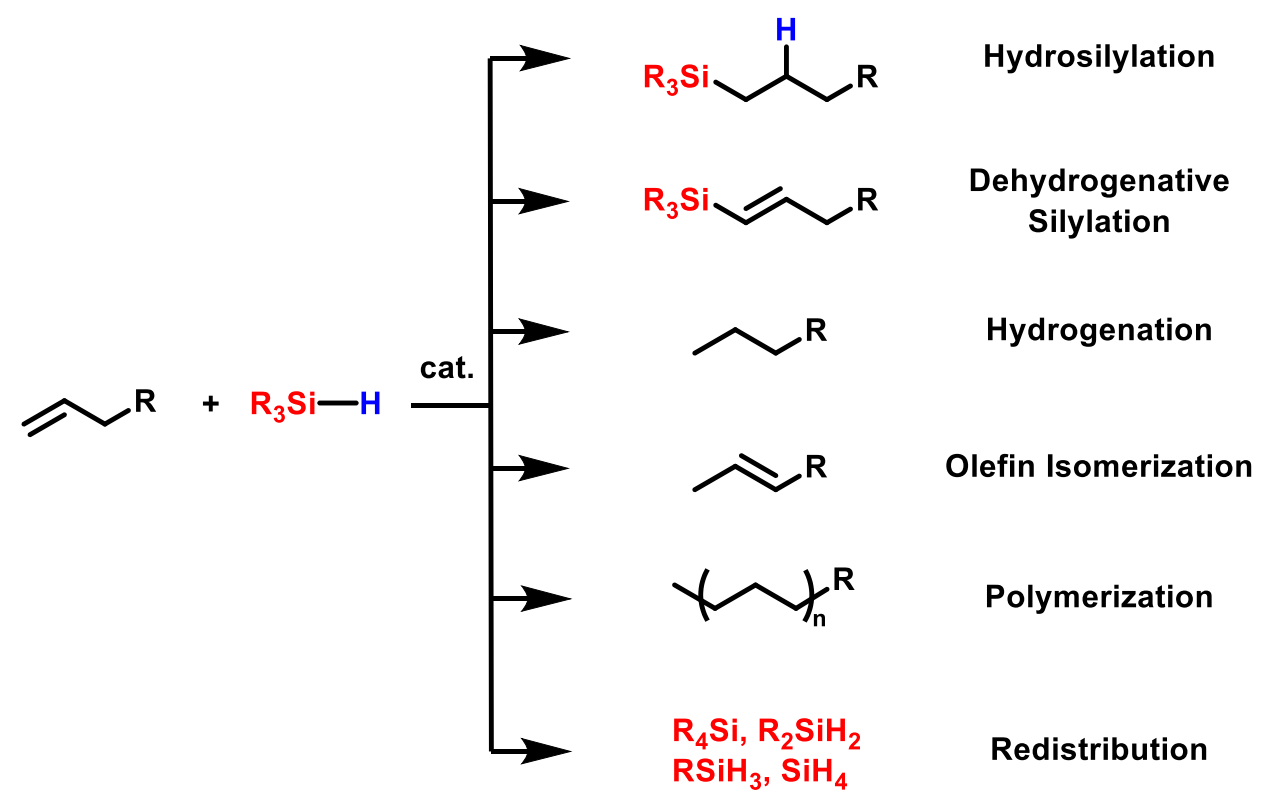

Figure 1.7: Iron-catalyzed hydrosilylation side reactions. . $5,111,113-116,120,169^{169}$ 


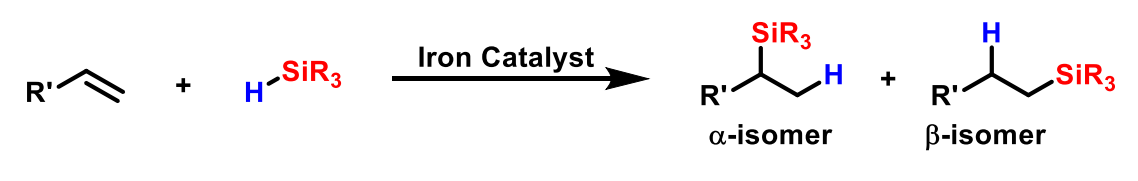

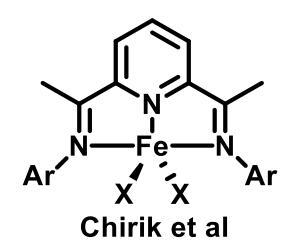<smiles></smiles>

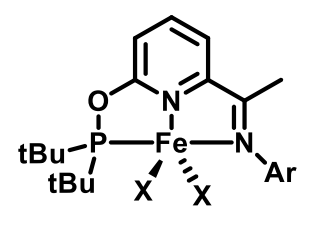

JACS 2004

Science 2012

Thomas et al

Adv. Syn. Catal 2014

Adv. Syn. Catal 2016

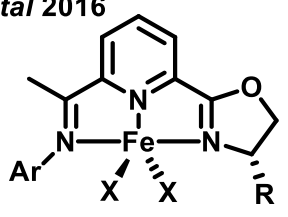

Lu et al ACIE 2015
Chirik et al Organometallics 2012

\section{Huang et al JACS 2013}<smiles></smiles>

Peng and Zhu Nat. Comm 2018

Figure 1.8: Iron-catalyzed hydrosilylation catalysts. ${ }^{95,111,113-116,120}$

A

Aliphatic Olefin Hydrosilylation<smiles>[R]CCC</smiles>

Hydrogenation

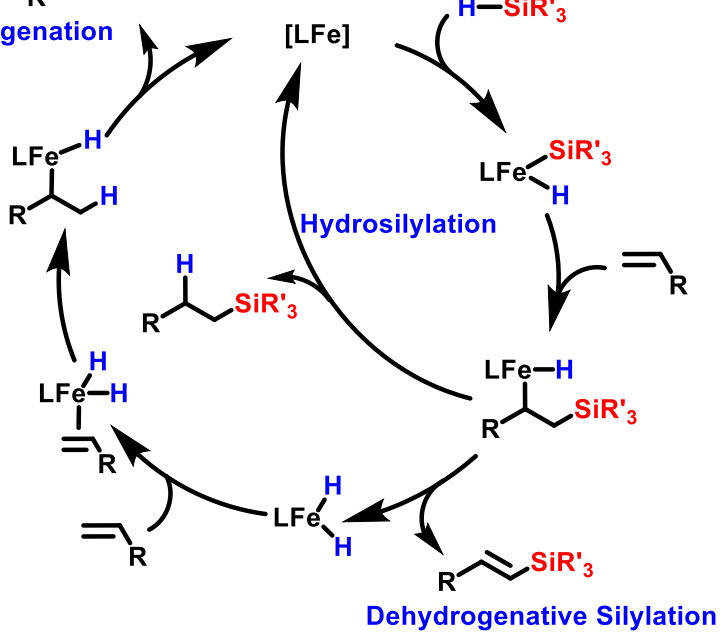

Aromatic Olefin Hydrosilylation

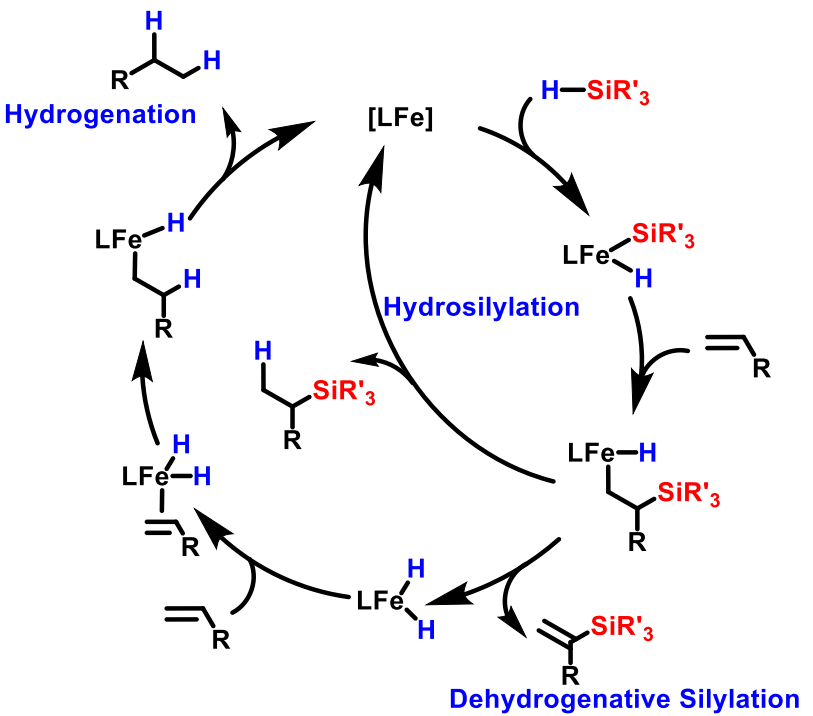

Scheme 1.9: Iron-catalyzed hydrosilylation. 95,111,113-116,120

${ }^{*}$ Reproduced using ChemDraw 
Another class of hydrofunctionalization, hydroboration, adds a borane $(B-H)$ across an unsaturated bond. The hydroboration of $\mathrm{C}=\mathrm{C}$ was first introduced by Brown and coworkers. ${ }^{171}$ The addition of B-H bonds with the aid of transition metals has expanded following work by Männig and Nöth using Wilkinson's catalyst. ${ }^{125}$ In 1990, Evans and Fu published a mechanistic study for the Rh-catalyzed hydroboration of olefins. ${ }^{126}$ The mechanistic studies revealed hydroboration has a mechanism quite similar to hydrosilylation, as shown in Scheme 1.9. One difference between the two classes of hydrofunctionalization reactions is the mechanism for hydroboration requires an activator (i.e. $\mathrm{Mg}$ or $\mathrm{NaBH}_{4}$ ) to reduce the iron center. Analogous to hydrosilylation, hydroboration also exhibits side reactions such as dehydrogenative boration and hydrogenation, although the products are observed in low yields. ${ }^{128}$ The development of a well-defined iron-ligand system, similar to those in Figure 1.9, aid in resolving a variety of side reactions along with regioselectivity and enantioselectivity degradation.

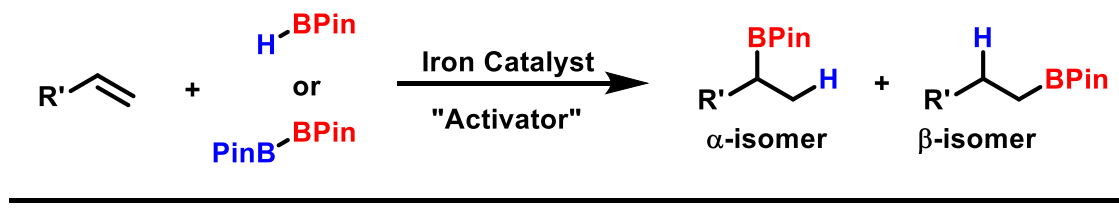

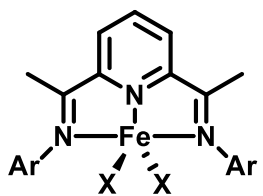

Chirik et al

Org. Lett 2013

Thomas et al Chem Comm. 2013

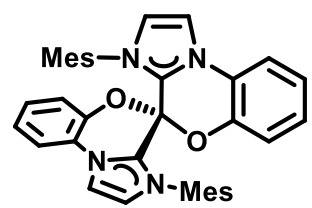

Thomas et al ACS Catal 2016

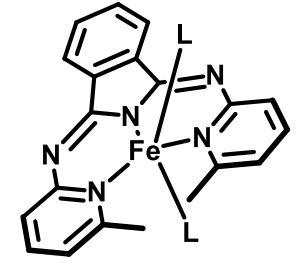

Szymczak et al ACS Catal 2015

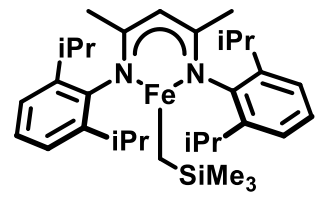

Webster et al Chem. -Eur. J. 2016

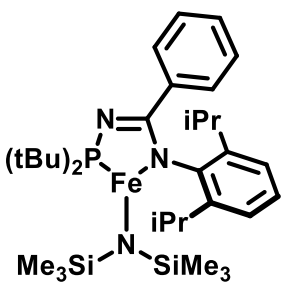

Turculet et al Chem. -Eur. J. 2014

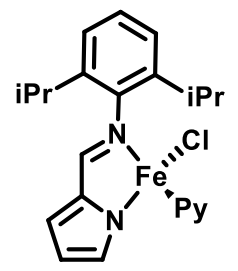

Gomes et al Catal. Sci. Technol 2019

Figure 1.9: Iron-catalyzed hydroboration catalysts. ${ }^{128-131,135,136,138}$ 
One of the most common industrial homogeneous hydrofunctionalization reactions is hydroformylation. ${ }^{143-149}$ Hydroformylation, also termed the "oxo" process, generates aldehydes from olefins and syn gas $\left(\mathrm{CO}\right.$ and $\left.\mathrm{H}_{2}\right)$. Most commonly, hydroformylation reactions are reported to be catalyzed by a Rh catalyst; however, there are only two recent publications using iron as a catalyst. ${ }^{143-149}$ Prior to 2018 , iron-catalyzed hydroformylation reactions were conducted at high temperatures and high pressures. ${ }^{9,10,147,149}$ Pertici and coworkers developed an iron(0) catalyst to catalyze the hydroformylation of 1 -hexene and styrene (Figure 1.10). ${ }^{147}$ These studies had limited mechanistic understanding, low reactivity, limited reaction scope, and disregarded any ligand effects. ${ }^{149}$ Chikkali and coworkers developed a detailed mechanistic study of ironcatalyzed hydroformylation, Figure 1.10. Their use of DFT studies, radical scavengers, discussions of Rh impurities in the iron precatalyst, cyclic-voltammetry (CV), and NMR studies led to the discovery of an iron(0) to iron(II) catalytic cycle over an iron(i) to iron(III) mechanism. Chikkali suggested that styrene and other vinylic arenes were able to undergo hydroformylation with the aid of acetic acid activation. ${ }^{149}$ Their mechanism is shown below, as shown in Scheme 1.10.

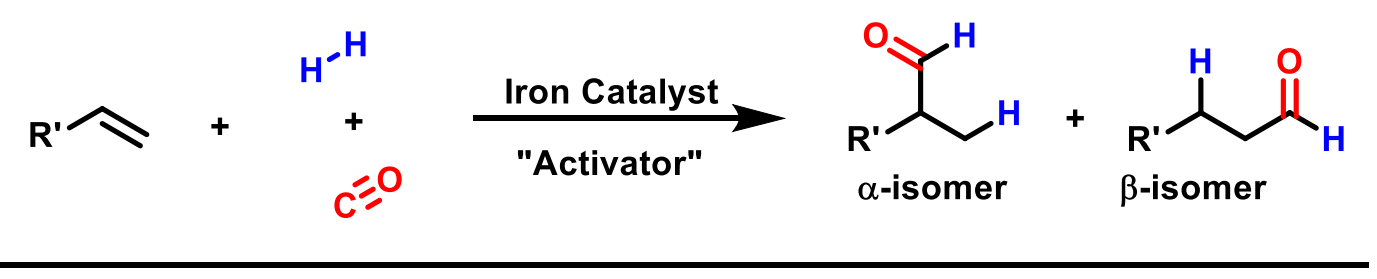

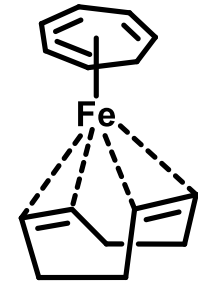

Pertici et al J.O.C 2000

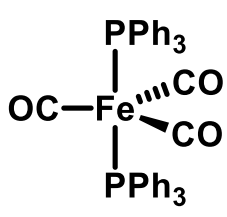

Chikkali et al JACS 2018

Figure 1.10: Iron-catalyzed hydroformylation catalysts. ${ }^{147,149}$ 


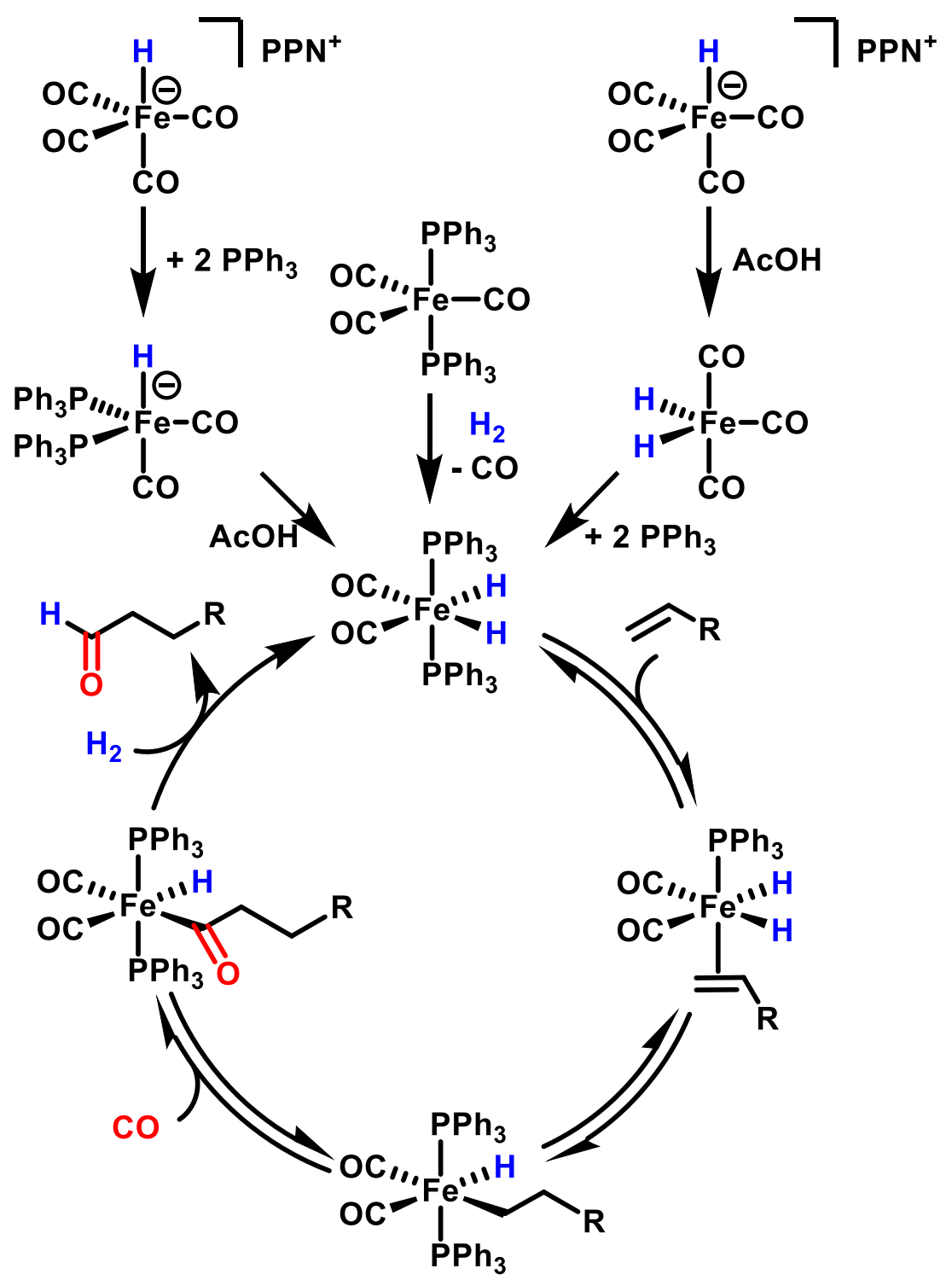

Scheme 1.10: Iron-catalyzed hydroformylation by an iron-hydride $\left(\mathrm{PPN}^{+}=\right.$ bis(triphenylphosphine iminium). *Reproduced using ChemDraw. ${ }^{149}$

The final hydrofunctionalization class discussed herein, hydrocarboxylation, is traditionally achieved by other means of catalysis utilizing pre-generated carbonyl containing substrates. Newer methods of formal hydrocarboxylation of vinyl arenes are demonstrated in Figure 1.11. $151,153,157-159,172-175$ The integration of $\mathrm{CO}_{2}$ as the carbon source for functionalization of alkenes/alkynes has its own challenges: 1) the stability of $\mathrm{CO}_{2}$ and 2) necessity for reductants and other additives for reactivity. More recent hydrocarboxylation reactions are either base metal-catalyzed cyclization carboxylation which 
generate carboxylated metallocycles or utilize the process of base metal-catalyzed hydrometallation carboxylation. ${ }^{151,157,161,162,176,177}$ The proposed mechanism of hydrocarboxylation, Scheme 1.11, proceeds through the following mechanism: reduction of the metal center and generation of a metal-hydride, insertion of an unsaturated bond (alkene/alkyne), insertion of $\mathrm{CO}_{2}$ through either direct insertion into the metal-alkyl or by transmetallation with an organometallic reagent (Grignard/zinc), then subsequent $\mathrm{CO}_{2}$ insertion, followed by protonolysis/metathesis/elimination. ${ }^{162}$ The most recent example in literature of iron-catalyzed formal hydrocarboxylation was published by Thomas initially in $2012 .{ }^{153}$ The iron-bis(imino)pyridine catalyzed transfer hydrometallation/carboxylation will be discussed further in subsequent chapters.

Hydroxycarbonylation

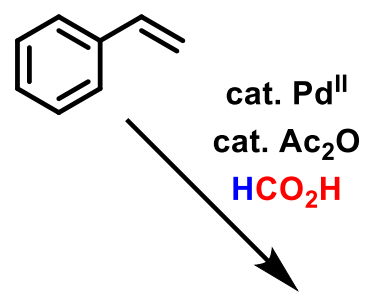

Hydrometallation Carboxylation

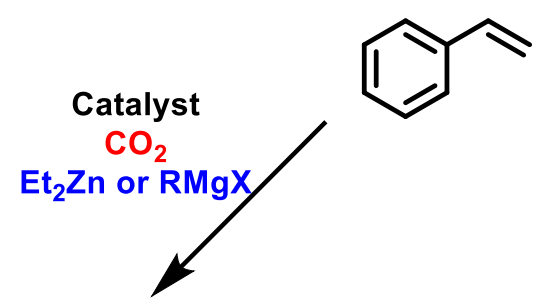<smiles>CC(C(=O)O)c1ccccc1</smiles>

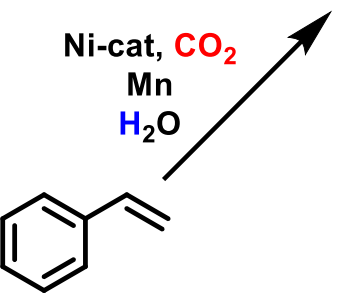

Reductive Nickel-catalyzed Hydrocarboxylation

Figure 1.11: Transition pathways. ${ }^{151,153,157-159,172-175}$

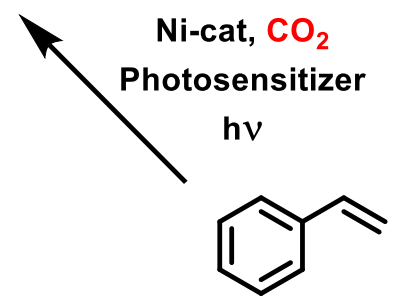

Photocatalytic Nickel-catalyzed Hydrocarboxylation

metal-catalyzed formal hydrocarboxylation 


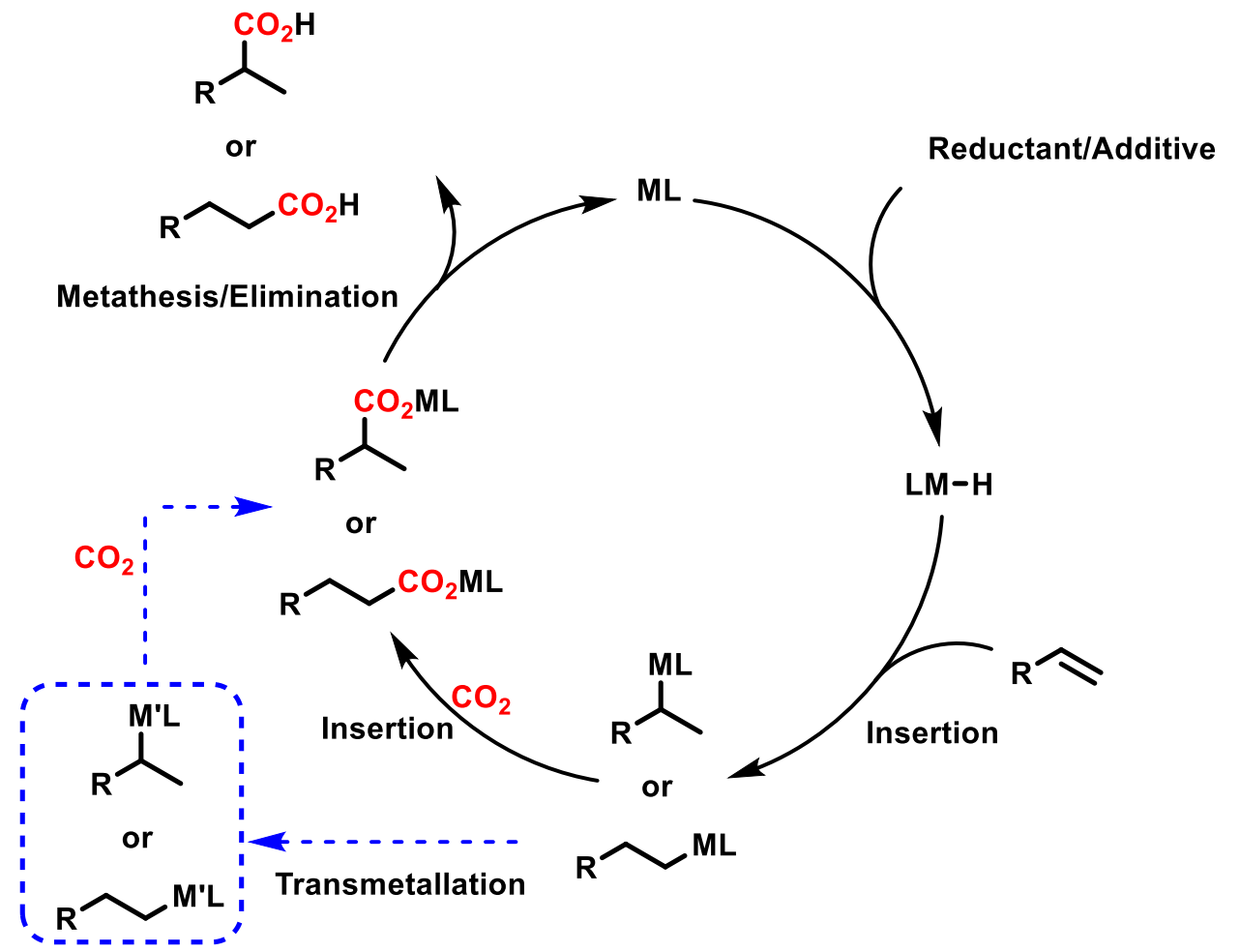

Scheme 1.11: Reproduced Transition Metal-catalyzed hydrocarboxylation. ${ }^{162}$

Finally, strides have been made in the category of transition metal-catalyzed difunctionalization or hetero(element)functionalization. The most common base metal catalyst for this type of transformation is $\mathrm{Cu}$, as described by Tsuji, Popp, and others. ${ }^{161,164-166}$ Some recent iron-catalyzed hetero(element)functionalization reactions are aminohydroxylation, sulfon-carbocyclization, oxysulfonylation, and carbonylationperoxidation-epoxidation, etc. ${ }^{178-182}$ These iron-catalyzed difunctionalization reactions generate more complex products from available olefins such as aliphatic alkenes and vinyl arenes. Figure 1.12 shows some of the work to date for iron-catalyzed difunctionalization methodologies. Mechanistic analyses in this class of homogeneous iron-catalysis are ongoing. One commonality exists however; these reactions are proposed to proceed through radical-based pathways. ${ }^{178-183}$ Although the body of literature for iron-catalyzed difunctionalization reactions is small, the expansion of this field is an area of interest given the multitude of $\mathrm{Cu}, \mathrm{Ni}$, and noble metal-catalyzed systems. 


\section{Aminohydroxylation/Amino-oxygenation}

Yoon (2010)<smiles>[R]C=C[R]</smiles>

$\mathrm{Fe}(\mathrm{acac})_{3}(5 \mathrm{~mol} \%)$

ACN, $0-23^{\circ} \mathrm{C}, 2-11 \mathrm{~h}$<smiles>[R]C1OC([Al])N([R6])[C@H]1[R]</smiles>

Xu (2014)<smiles>[R3]C=C([R])[R]</smiles>

$\mathrm{Fe}(\mathrm{OTf})_{2}(10 \mathrm{~mol} \%)$

Tridentate Ligand

DCM/ACN, 4 Á MS

$-15^{\circ} \mathrm{C}, 2 \mathrm{~h}$

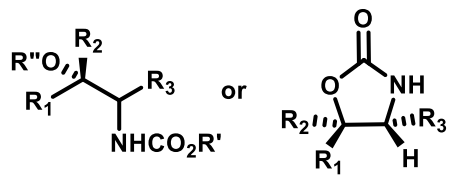

$61-85 \%$

\section{Amino/Carbosilylations}

Li and Luo (2017)

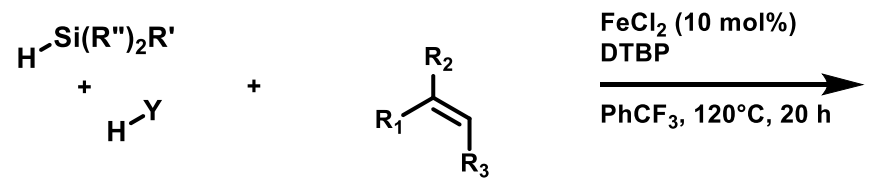

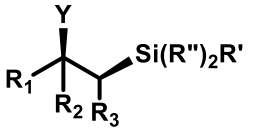

$35-72 \%$

\section{Decarboxylative-Alkyl Etherification}

Bao (2017)

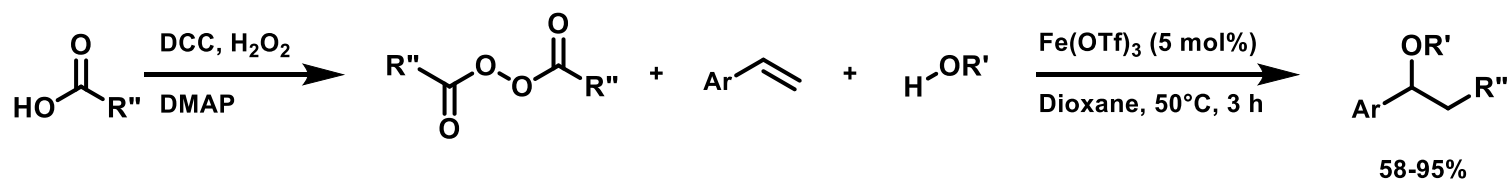

\section{Carbonylation-Peroxidation-Epoxidation}

Li (2011)

$\mathrm{H}_{\mathrm{R}^{\prime \prime}}^{\mathrm{O}^{-}}+\mathrm{H}^{-O O \mathrm{R}^{\prime}}+\underset{\mathrm{R}_{1}}{\stackrel{\mathrm{R}_{2}}{\mathrm{FeCl}_{2}(2.5 \mathrm{~mol} \%)}}$

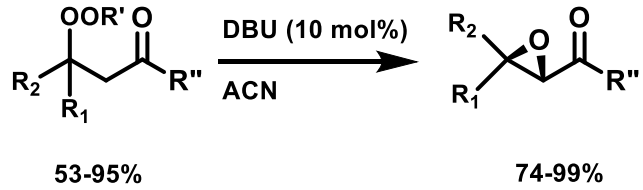

\section{Sulfon-carbocyclization}

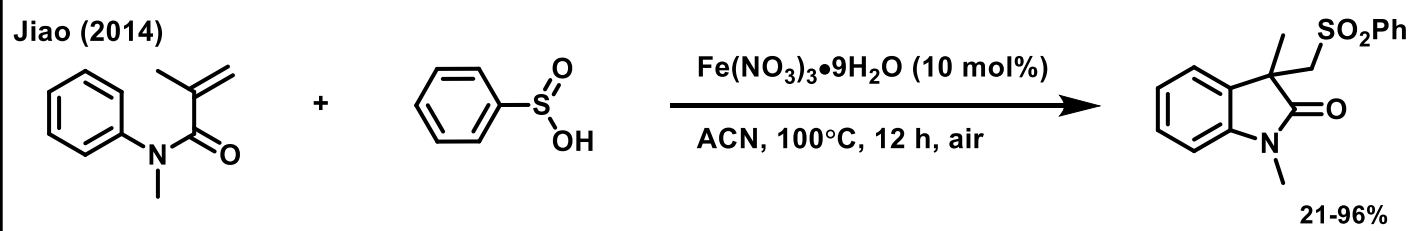

Figure 1.12: Iron-catalyzed difunctionalization pathways. ${ }^{178-183}$ 


\subsection{Conclusions}

The development of iron-catalyzed homogeneous catalysis within the past decade has prospered by the expansion of multiple classes of reaction methodology. While iron is becoming a commonly used transition metal for catalysis owing to its abundance, the development of mechanistic studies across organometallic methodologies is relatively sparse. Identification and characterization of key intermediates in these synthetic pathways has led to the extension in catalysis and paved the way for new mechanistic insights into decades old processes. From enzymatic mimics to simple iron salts and everything in between, homogeneous iron catalysis has been a class of organometallic transformations that has been evolving over the years, yet still consists of a variety of routes for mechanistic discovery. The succeeding chapters will describe recent advances in the kinetic and mechanistic analyses within the methodology of iron-catalyzed formal hydrocarboxylation via hydromagnesiation. 


\section{Chapter 2. Iron-catalyzed hydromagnesiation}

\subsection{Introduction}

Over the past decade, there have been an abundance of advances in hydrofunctionalization reactions catalyzed by base metals. The process of reductive functionalization of alkenes/alkynes have been especially prominent with important examples such as hydroboration ${ }^{116-118,122,127,129,132-134,137}$, hydrosilylation ${ }^{104,111,114,116-124}$ and hetero(element)-functionalization ${ }^{164-167}$ which are described in more detail in Chapter 1.6. An under-explored class of alkene reductive functionalization proceeds through transfer hydrometallation, specifically hydromagnesiation $11,12,153,157,184-188$ or hydrozincation ${ }^{151,189-192}$, unfortunately these systems suffer from limited substrate scope and overall regioselectivity. Nevertheless, this class of reactions provide synthetic utility by providing more complex, valuable organometallic reagents. One such class of organometallic reagent, (aryl)ethyl Grignard, shown in Scheme 2.1 A, can be generated through transfer hydrometallation of styrene or traditional Grignard synthesis using 1bromo-1-phenylethane. (Aryl)ethyl Grignards, much like other Grignard reagents, are amenable to electrophilic substitution with a variety of substrates described in Scheme 2.1B.

Substitution with $\mathrm{CO}_{2}$, an important and underutilized $\mathrm{C}_{1}$ feed stock, yields aryl propanoic acids a common pharmacophore in NSAIDs. ${ }^{193}$ Initial analyses in hydrometallation reactions namely with the generation of a neopentyl tethered ibuprofen derivative, "neoprofen", displayed inconsistencies in known trends for reductive carboxylation of styrenes. By collaborating with the Dudley lab at West Virginia University, the synthesis of these aryl propanoic acids were conducted under 5 different methodologies for comparison. Interestingly, all forms of formal hydrocarboxylation via transfer hydrometallation resulted in low to moderate yields of "neoprofen" in comparison to 3,4-dimethylstyrene and 4-methylstyrene (Figure 2.1). The trend in lower yields and sparse mechanistic studies for transfer hydrometallation has introduced the need to have a better understanding of this catalytic process overall. ${ }^{12,13,153,170,194,195}$ 
A

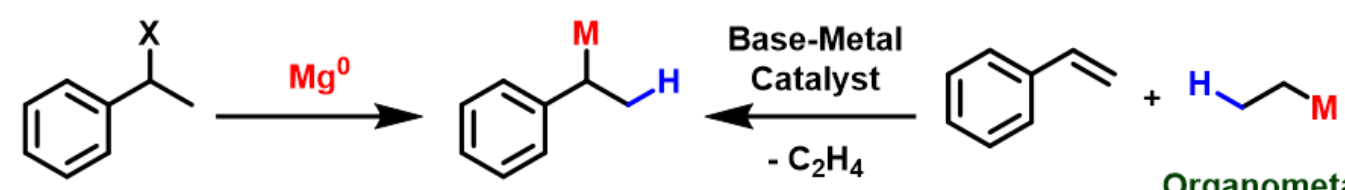

Organometallic Grignard or zinc reagent

B

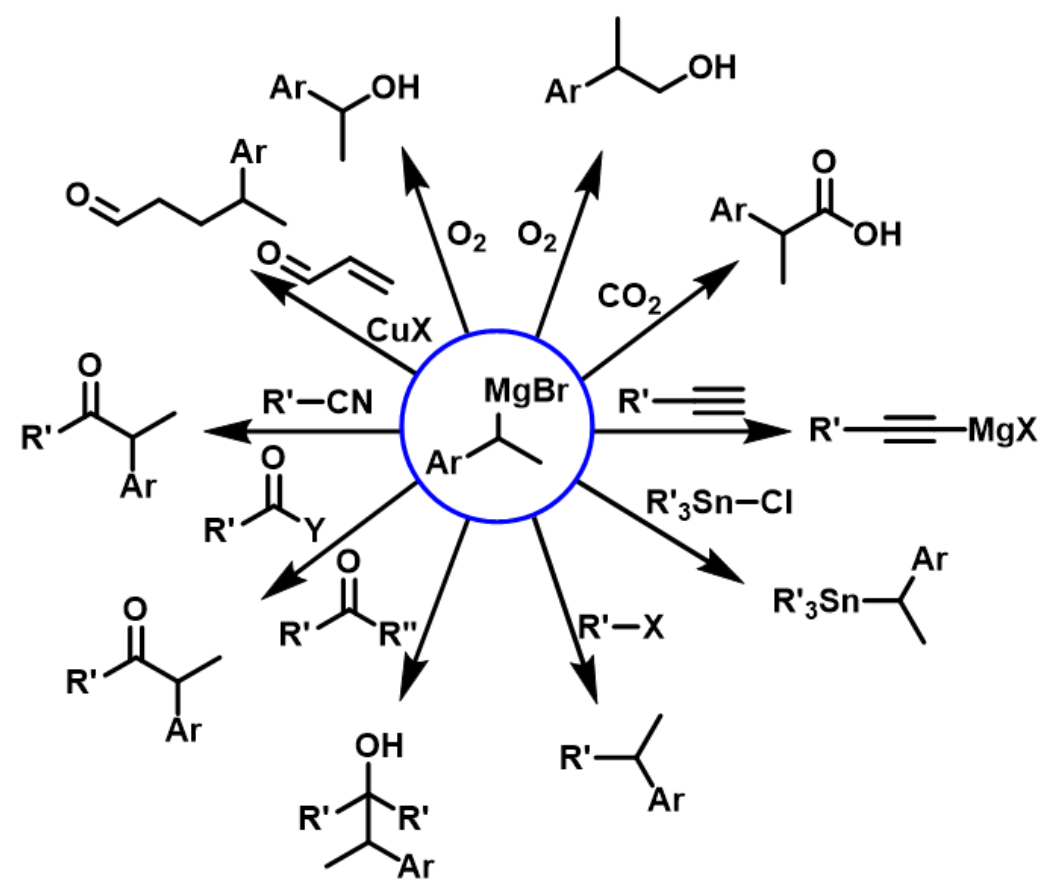

Scheme 2.1: Synthetic utility of (aryl)ethyl Grignards. A.) Synthesis of Grignard. B.) Utility of Grignard reagents.

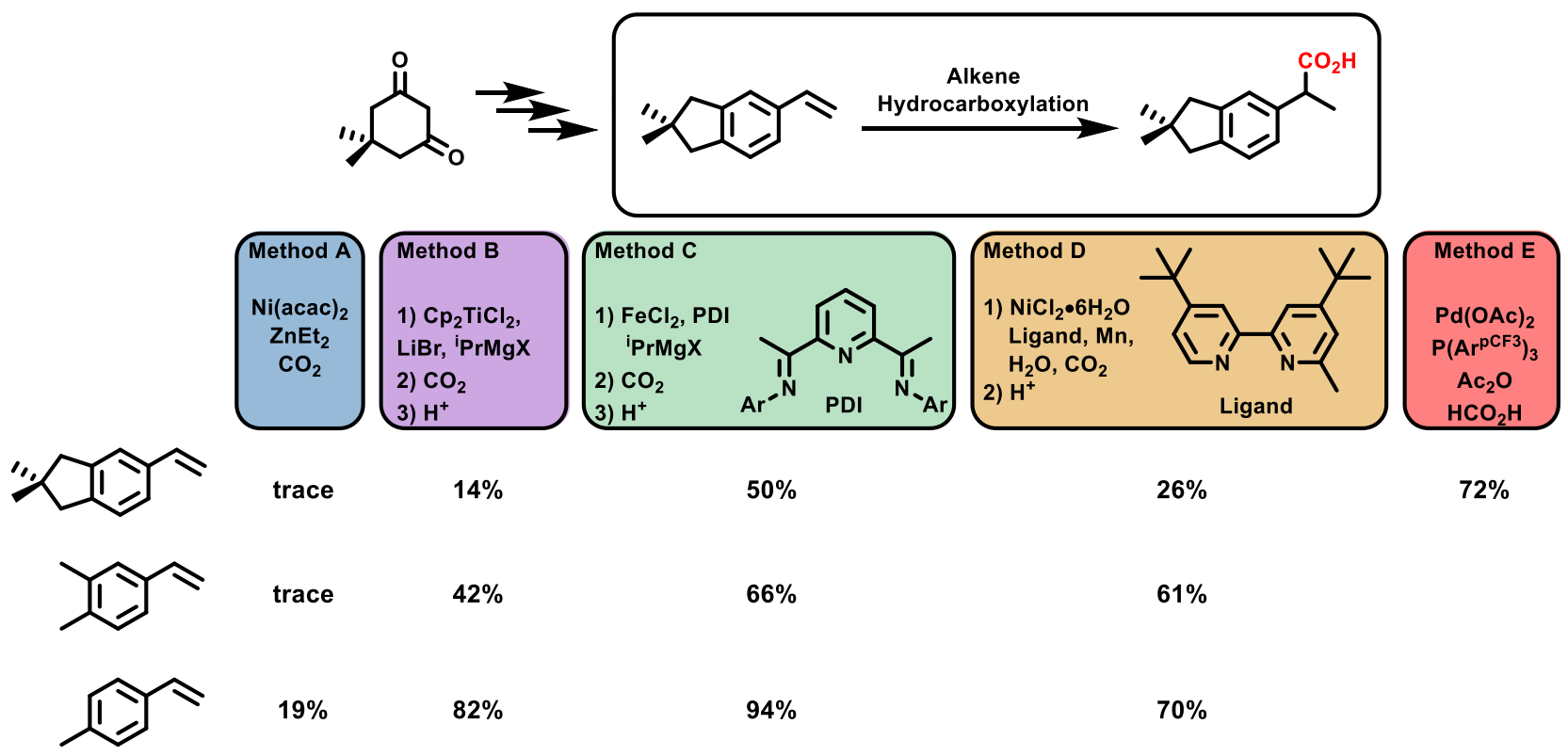

Figure 2.1: Hydrocarboxylation of neopentylene fused styrene yielding "neoprofen". 
Kochi described transfer hydrometallation to proceed through "the basic transformation of metal-alkyls," Scheme 2.2A, omitting the likely mechanistic complexity often observed with reactions of organometallic reagents. ${ }^{13}$ Only recently have significant strides been made to elucidate the mechanism of these transformations. In 2012, Thomas utilized an in situ generated catalyst, 2,6-bis[1-(2,6-diisopropylphenyimino)ethyl]pyridine iron(II) dichloride (iron-PDIPr), to achieve hydromagnesiation of electron-rich and neutral styrene derivatives (Scheme 2.3A). ${ }^{153}$ The subsequent mechanistic analysis in Scheme 2.2B revealed: 1) complex kinetic regimes were evident for the catalyst and each substrate; 2) DHT (Scheme 2.2A steps 2-3), not BHE/MI (Scheme 2.2A steps 5-6), is the likely elementary catalytic step based upon deuterium labeling studies (Scheme 2.3B); 3) the resting state, as well as off cycle catalytic intermediates, are characterized as iron(0)-ate species. ${ }^{170,195} \mathrm{Up}$ to this point in the literature, the mechanistic investigations described above have not defined the further complex kinetic behavior nor do they take into account the role of the formation of the linear regioisomer. In this chapter, in situ infrared spectroscopy will be used to aid in the mechanistic developments of iron-catalyzed hydromagnesiation. A detailed kinetic analysis and numerical modeling of the ironcatalyzed hydromagnesiation reaction will also be utilized to offer an explanation to the kinetic complexity observed and build a mechanistic foundation for the future expansion of iron-catalyzed transfer hydrometallation reactions. 
A.

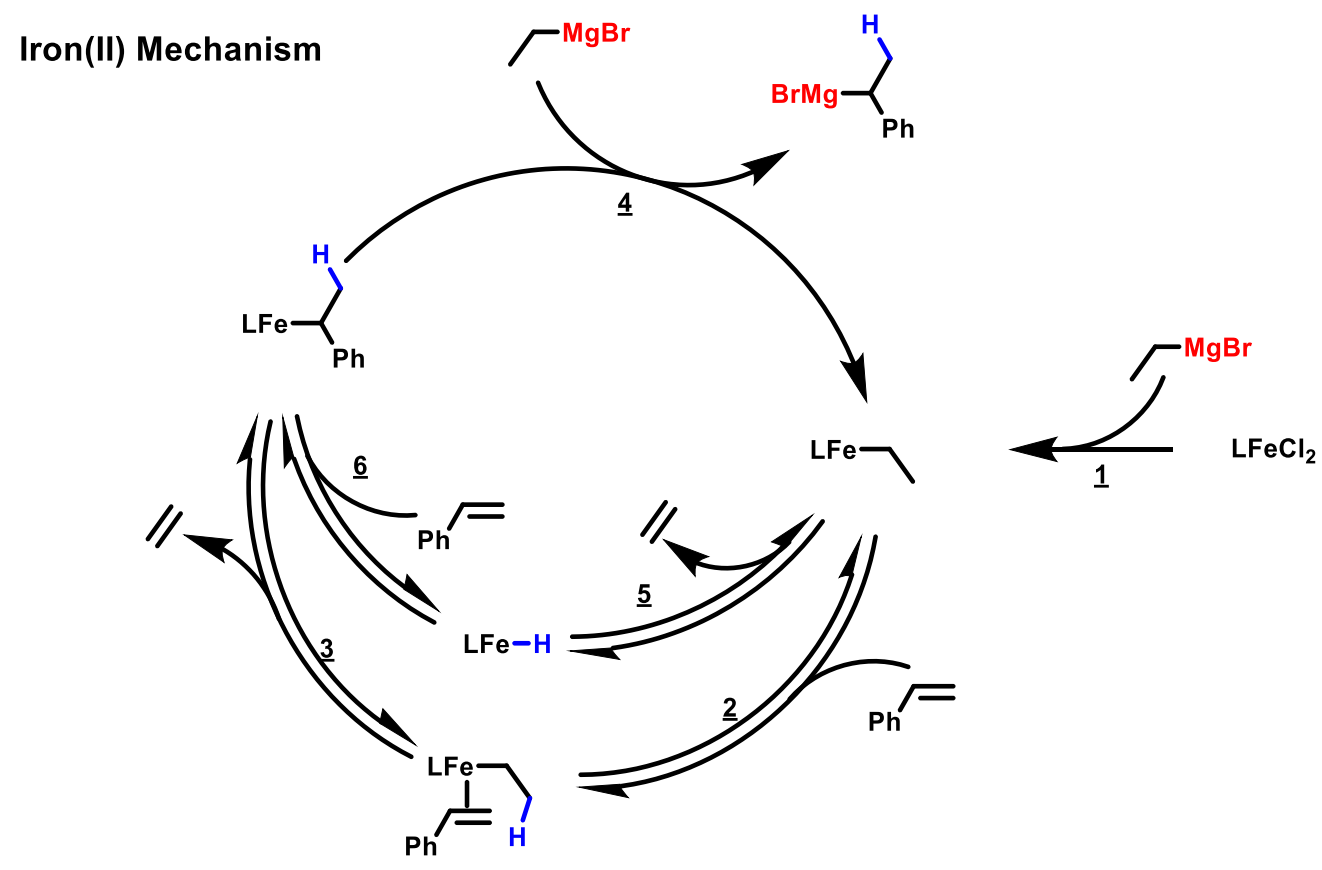

B.
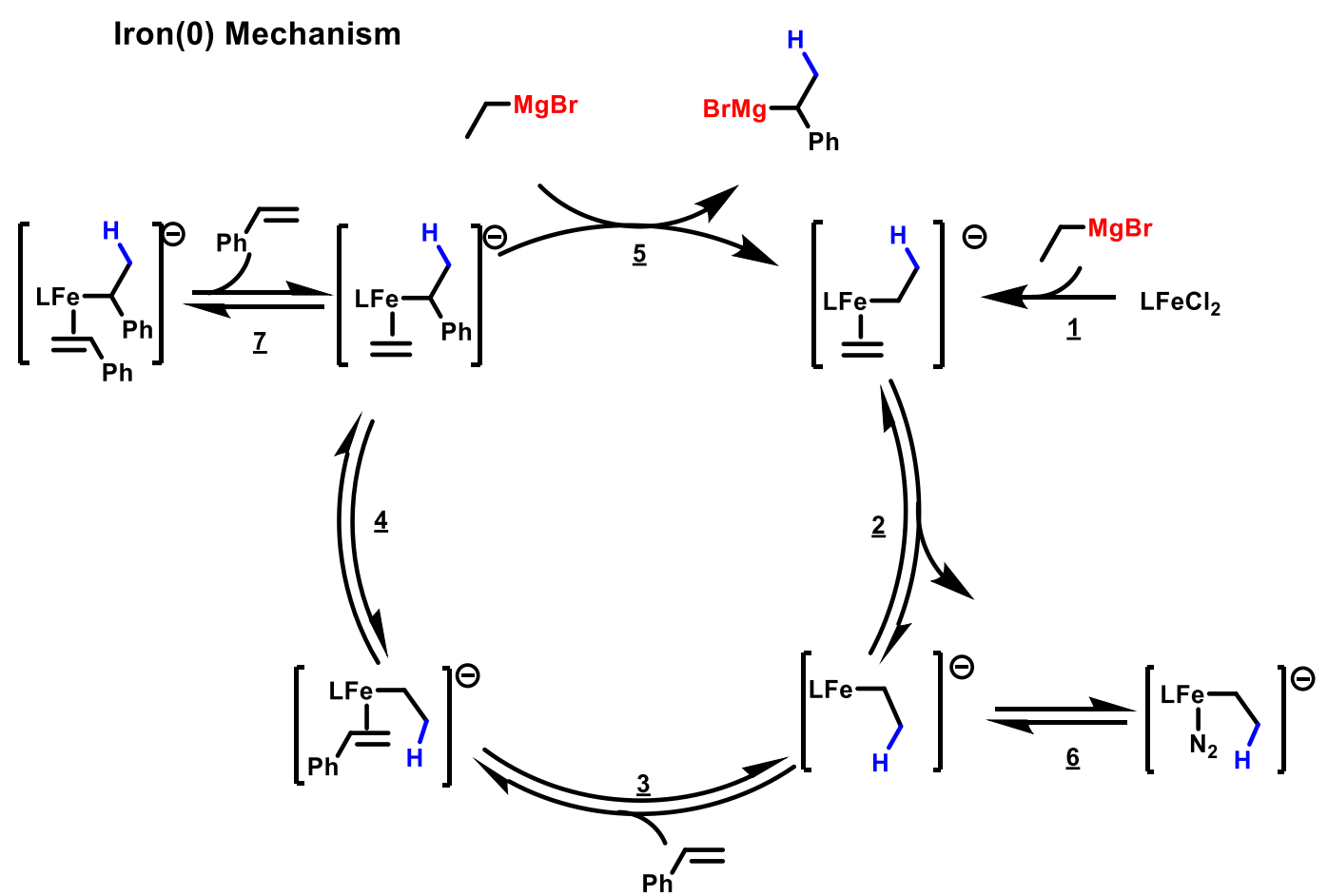

Scheme 2.2: A) Thomas/Kochi iron(II) mechanism for transfer hydrometallation B) Thomas/Neidig iron(0) mechanism. *Reproduced in ChemDraw ${ }^{13,153,195}$ 
A.<smiles>C=Cc1ccccc1-c1ccccc1</smiles>

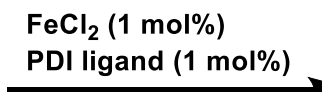

favored

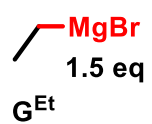

THF, $\mathbf{N}_{2}$ (1 atm), rt, $1 \mathrm{~h}$
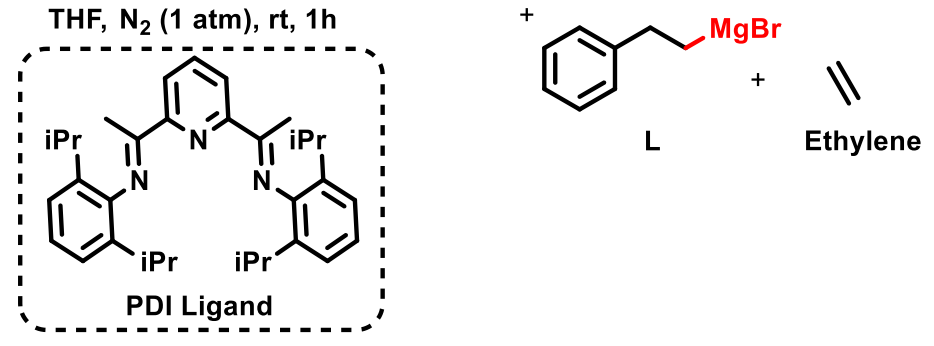

B.
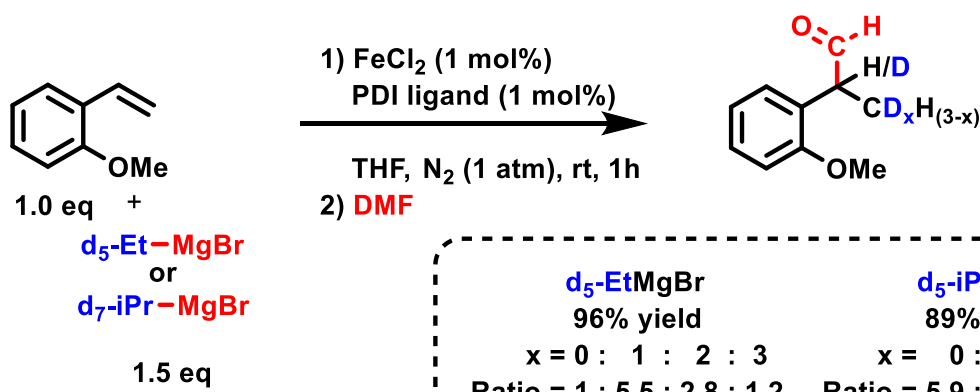

2) $\mathrm{DMF}$

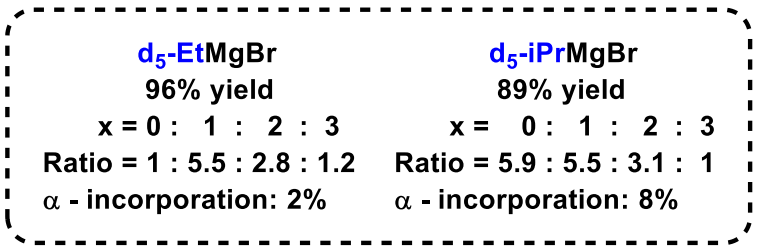

Scheme 2.3: A) Thomas' Hydromagnesiation, B) Thomas' deuterium studies. Conditions: anhydrous $\mathrm{FeCl}_{2}(1 \mathrm{~mol} \%), \mathrm{PDI}^{\mathrm{PPr}}$ ligand (1 mol\%), S (1.0 eq), GX $(1.5$ eq), THF, N2 (1 atm), rt, 1 h. Quench: 1) $\mathrm{CO}_{2}$ (dry) or DMF, rt, 1h; 2) $1.0 \mathrm{M} \mathrm{HCl}$, rt, overnight. ${ }^{153,195}$

\subsection{Results}

The mechanistic analysis of iron-catalyzed hydromagnesiation of styrene (S) were initiated by examining Thomas' conditions with cyclopentyl Grignard (GCYP) and isolated (PDIPr) $\mathrm{FeCl}_{2}$ using a ReactIR ic15 system by Mettler Toledo (Scheme 2.4). GCYP was previously shown by Thomas to be an efficient sacrificial Grignard, leading to high yields of branched Grignard product, BR, albeit with slightly lower regioselectivity compared to ethyl Grignard, $G^{E t} .{ }^{153}$ Therefore, simplification of kinetic reaction analysis was achieved by using GCYP owing to the formation of cyclopentene, CYP, a soluble alkene compared to gaseous ethylene. The lower regioselectivity when using $\mathrm{G}^{\mathrm{CYP}}$ also allows for infrared characterization of linear Grignard, L. The identification of the infrared spectral signatures for $B R$ and $L$ were assigned as 1590 and $1602 \mathrm{~cm}^{-1}$, respectively (Figure 2.2). Through spectral deconvolution described in the Experimental Appendix section E.4, the reaction timecourses for both regioisomers were obtained, shown in Figure 2.3. Exponential growth 
of both products allows for a full characterization of the hydromagnesiation process using both initial rate and observed rate determination.
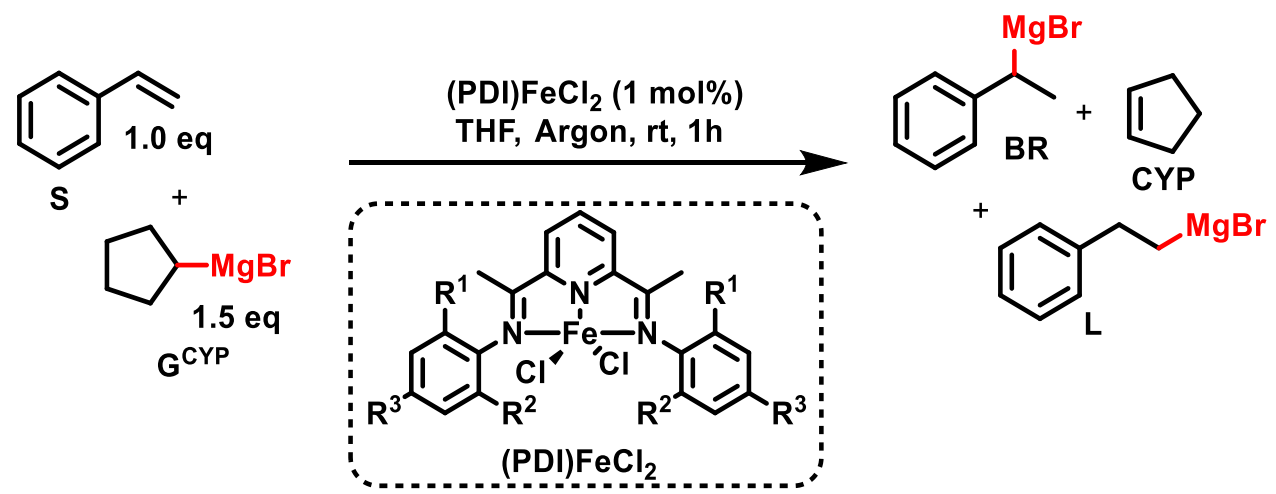

Scheme 2.4: Iron-catalyzed hydromagnesiation with cyclopentylMgBr, GCYP. Conditions: anhydrous (PDIPr) $\mathrm{FeCl}_{2}(1 \mathrm{~mol} \%), \mathrm{S}$ (1.0 eq, $\left.0.87 \mathrm{mmol}, 0.3 \mathrm{M}\right), \mathrm{GCYP}^{\mathrm{CY}}(1.5$ eq, $1.3 \mathrm{mmol}, 0.43 \mathrm{M}$ ), THF, argon (1 atm), rt, $1 \mathrm{~h}$. Quench: 1) $\mathrm{CO}_{2}$ (dry), rt, $1 \mathrm{~h}$; 2) 1.0 $\mathrm{M} \mathrm{HCl}$, rt, overnight.
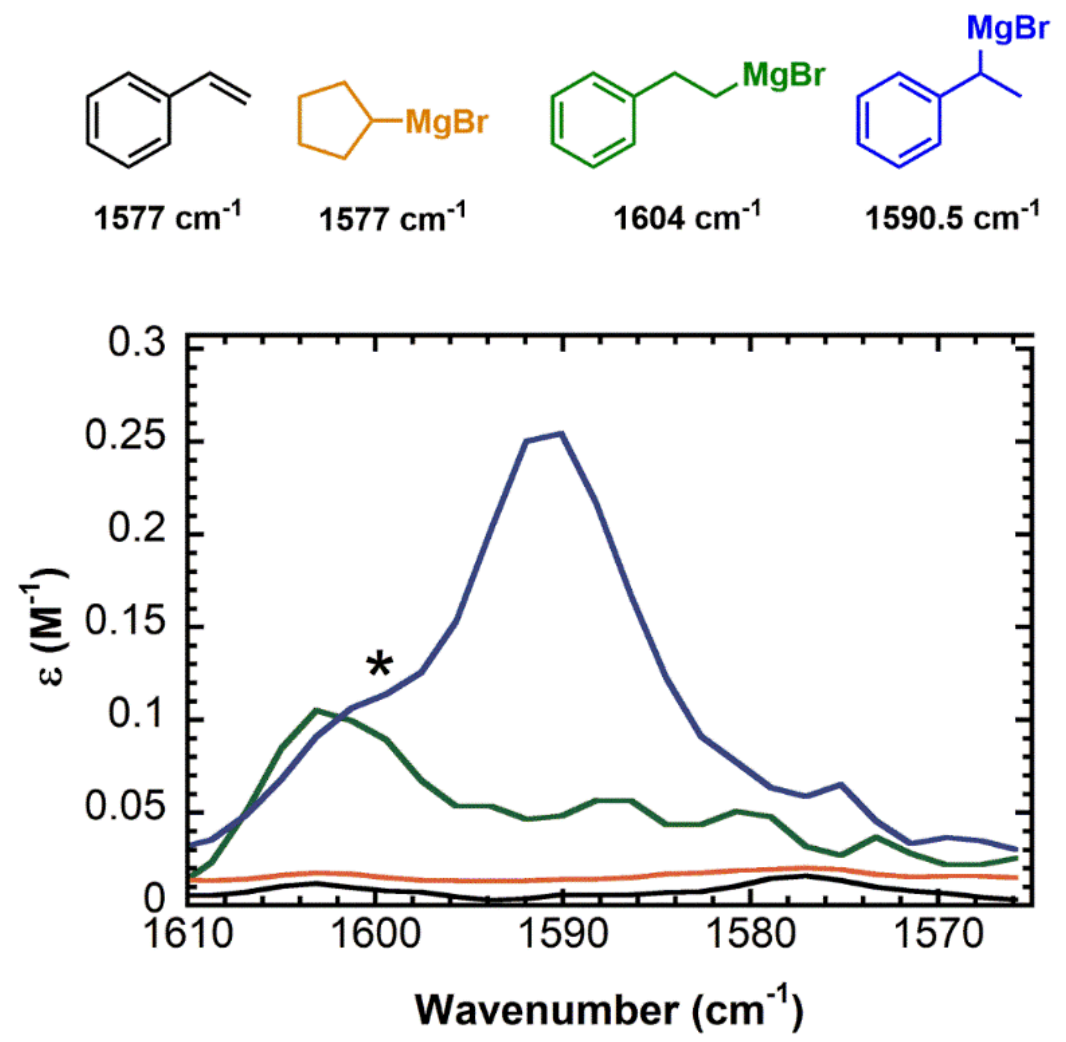

Figure 2.2: Frequency analysis of the absorbances of the starting material and the product from Scheme 2.4. Normalized extinction coefficient vs wavenumber. ("small shoulder acquired from 1-bromo-1-phenylethane overlap) 

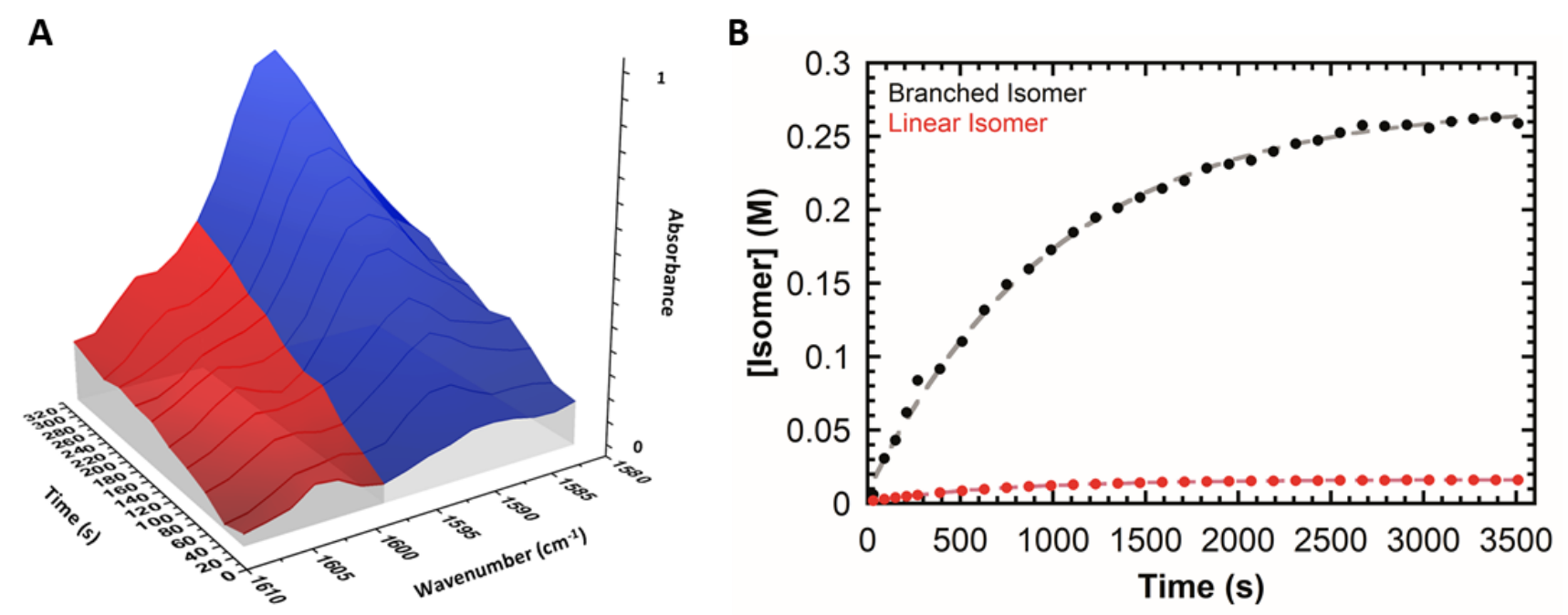

Figure 2.3: Reaction time courses. A) 3D time course over the first 5 minutes of reactivity; B) 2D time course fitted to an exponential.

Kinetic analyses of the hydromagnesiation reactions were performed by independently varying the concentration of reactants, catalyst, and cyclopentene. The initial rate and observed rate results, as described in Figure 2.4, reveal kinetic behavior that is rather complex. Namely, initial rates indicating non-linear kinetic behavior for both catalyst and GCYP, while linear behavior with a non-zero intercept was observed for styrene, S. The analysis of the observed rates increased the overall reaction kinetics complexity. These analyses indicated linear kinetic behavior for catalyst and inhibitory behavior for both GCYP and $S$ at low concentrations. When concentrations of the substrates are similar or in excess, the observed rate behavior changes with GCYP displaying a non-linear behavior and $S$ demonstrating no change in rate. Based upon Thomas' previous studies, a change in observed kinetic behavior may suggest that transmetallation to generate a new Grignard product is the turnover limiting step, TLS. ${ }^{153,170,195}$ Further analysis is needed to confirm this assumption. Lastly, addition of exogenous CYP, up to 15 equivalents relative to $S$, shows no effect on initial rate or observed rate (Figure 2.5). 
Initial Rate

A

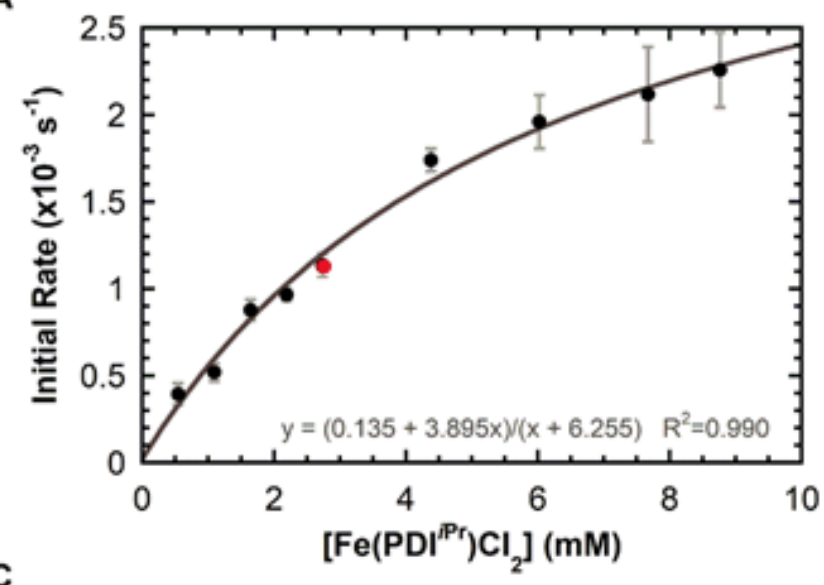

C

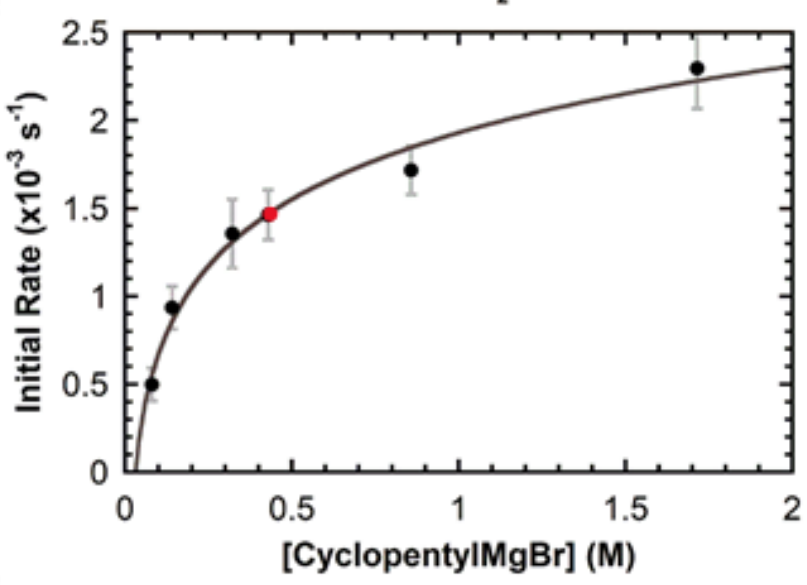

E

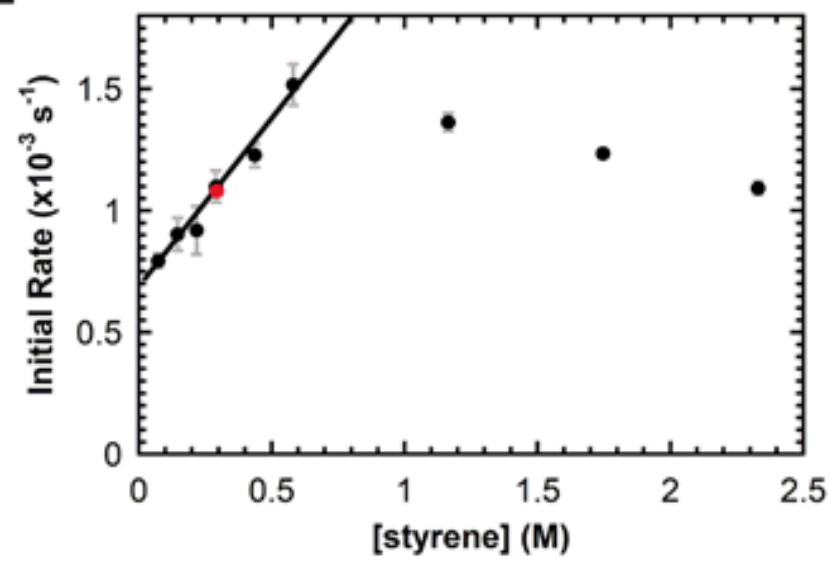

Observed Rate

B
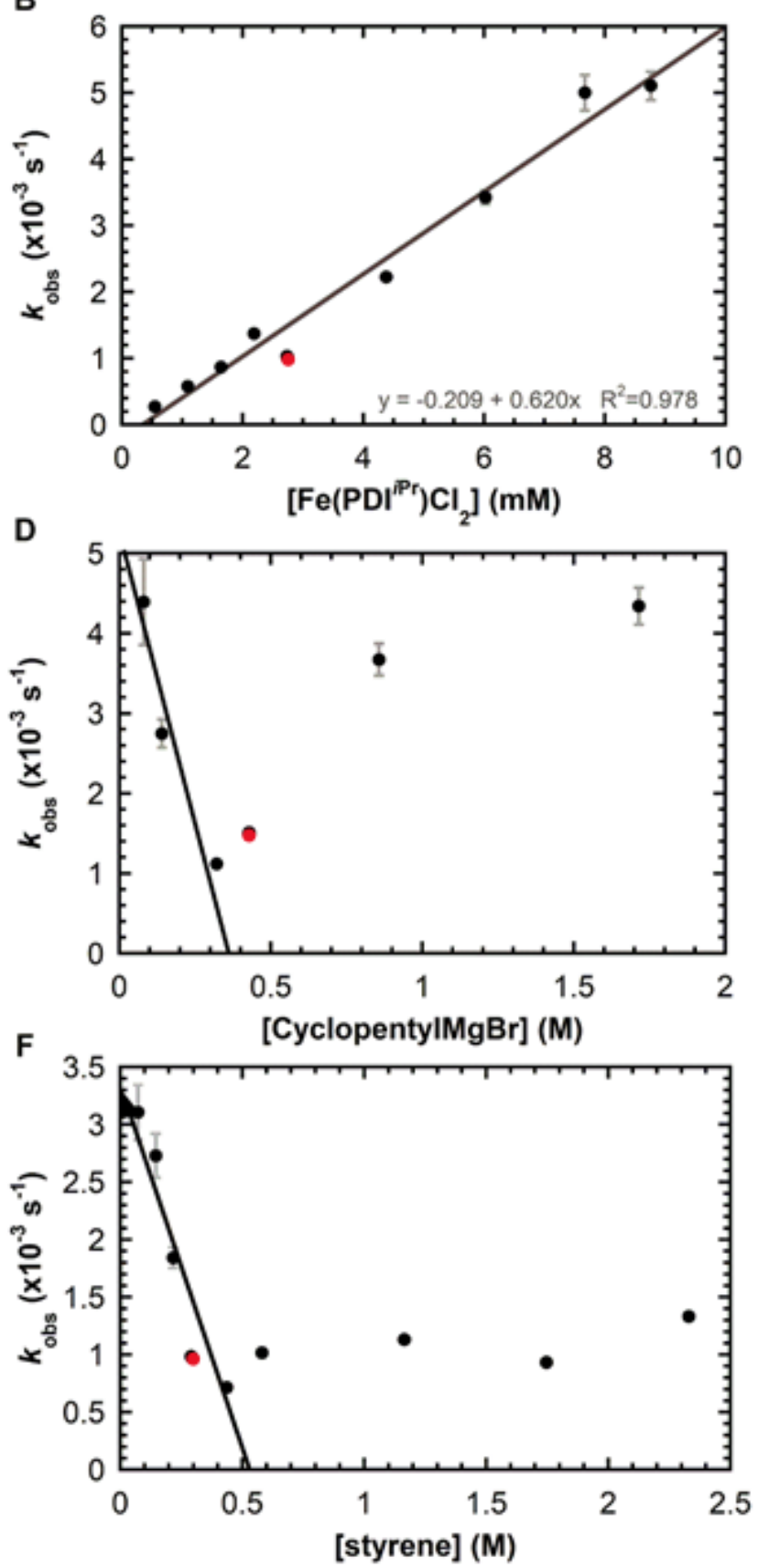

Figure 2.4. Concentration dependencies of initial and observed rates for catalyst and substrates. Points in red represent standard reaction conditions (see Scheme 2.4). 


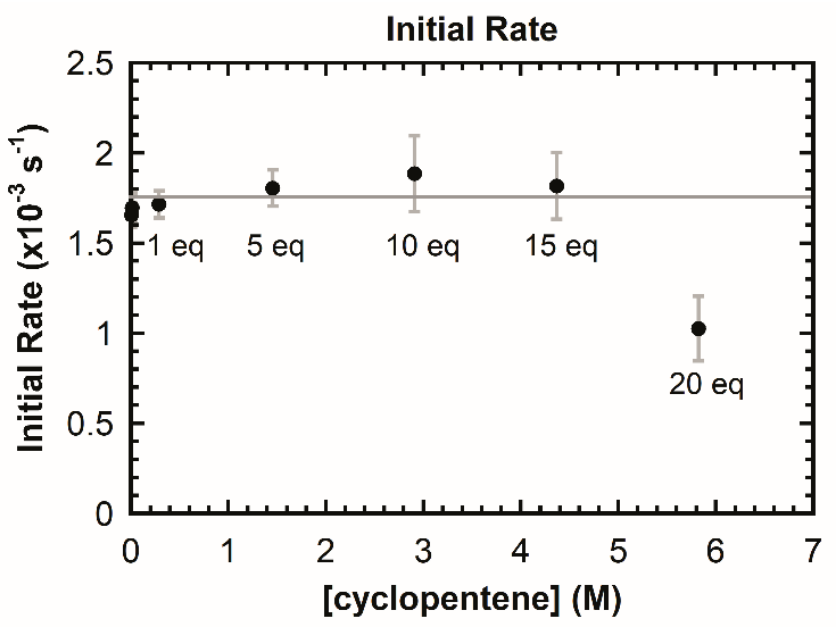

Figure 2.4: Initial rate dependence of cyclopentene: [(1-phenylethyl) $\mathrm{MgBr}]$ vs time. Conditions: 0.291 M S; 2.91 mM (PDIPr)FeCl2; 0.434 M G; x mol\% CYP; 1 hour; THF; rt.

Numeric simulation using COmplex PAthway Simulator (COPASI) was used given the complexity of the kinetic behavior to determine probable mechanisms of transfer hydromagnesiation. ${ }^{196}$ Parameter estimations using evolutionary programming and Livermore Solver for Ordinary Differential Equations (LSODA) deterministic timecourse analysis allowed for comparison to experimentally collected timecourses. With simulation cost taken into consideration, a collection of ten timecourses were chosen to reflect various experimental regimes and used for numeric fitting. The Experimental Appendix fully describes the details of numeric modeling and the use of Cohen's $\mathrm{k}$ for assessing agreement between numerical and experimental timecourses (Table 2.1).

Table 2.1: Cohen's Kappa agreement definition

\begin{tabular}{c|l}
\multicolumn{2}{c}{$\boldsymbol{\kappa}=\frac{\text { observed } \%-\text { expected } \%}{1-\text { expected } \%}$} \\
\hline $\boldsymbol{\kappa}<\mathbf{0}$ & Minimal agreement \\
$\mathbf{0 . 1}<\boldsymbol{\kappa}<\mathbf{0 . 2}$ & Slight agreement \\
$\mathbf{0 . 2 1}<\boldsymbol{\kappa}<\mathbf{0 . 4}$ & Fair agreement \\
$\mathbf{0 . 4 1}<\boldsymbol{\kappa}<\mathbf{0 . 6}$ & Moderate agreement \\
$\mathbf{0 . 6 1}<\boldsymbol{\kappa}<\mathbf{0 . 8}$ & Substantial agreement \\
$\mathbf{0 . 8 1}<\boldsymbol{\kappa}<\mathbf{0 . 9 9}$ & Near-perfect /excellent agreement \\
$\boldsymbol{\kappa}=\mathbf{1}$ & Perfect agreement
\end{tabular}


Table 2.2: Calculated rate constants from COPASI for Scheme 2.2A direct $\beta$-hydride transfer mechanism

\begin{tabular}{l|lll} 
Rxn \# & Conditions & kforward & kreverse \\
\hline 1 & $P+G=C G$ & 73.10 & $3.39 \times 10-5$ \\
2 & $C G+S=C G S$ & 1.859 & $1.0 \times 10-6$ \\
3 & CGS = CP + CYCLOPENTENE & 1439.75 & 568974 \\
4 & $C P+G=C G+B R$ & 6.841 & $6.5 \times 10-6$
\end{tabular}

Table 2.3: Calculated rate constants from COPASI for Scheme 2.2A $\beta$-hydride elimination/migratory insertion mechanism

\begin{tabular}{l|lll}
\multicolumn{2}{l}{ Rxn \# Conditions } & kforward & kreverse \\
\hline 1 & $P+G=C G$ & 826281 & 53040.3 \\
5 & $\mathrm{CG}=\mathrm{CGH}+\mathrm{CYCLOPENTENE}$ & 3119.42 & 2.861 \\
6 & $\mathrm{CGH}+\mathrm{S}=\mathrm{CP}$ & 688.19 & 0.726 \\
4 & $\mathrm{CP}+\mathrm{G}=\mathrm{CG}+\mathrm{BR}$ & 2.048 & 346090
\end{tabular}

Table 2.4: Calculated rate constants from COPASI for Scheme 2.2B $\beta$-hydride transfer 2019 mechanism

\begin{tabular}{l|lll}
\multicolumn{2}{l}{ Rxn \# Conditions } & kforward & kreverse \\
\hline 1 & $\mathrm{P}+3^{*} \mathrm{G}->\mathrm{CG} \cdot \mathrm{CYP}$ & $7.53 \mathrm{E}+3$ & \\
2 & $\mathrm{CG} \cdot \mathrm{ET}=\mathrm{CG}+\mathrm{CYCLOPENTENE}$ & 0.519 & $3.87 \mathrm{E}+4$ \\
3 & $\mathrm{CG}+\mathrm{S}=\mathrm{CGS}$ & $2.07 \mathrm{E}+3$ & 0.00418 \\
4 & $\mathrm{CGS}=\mathrm{CP}$ & 0.906 & $3.71 \mathrm{E}-6$ \\
5 & $\mathrm{CP}+\mathrm{G}=\mathrm{CG} \cdot \mathrm{CYP}+\mathrm{BR}$ & 15.8 & $5.37 \mathrm{E}-5$ \\
6 & $\mathrm{CG}+\mathrm{N} 2=\mathrm{CG} \cdot \mathrm{N} 2$ & 81.3 & 3.07 \\
7 & $\mathrm{CP}+\mathrm{S}=\mathrm{CP} \cdot \mathrm{S}$ & $4.67 \mathrm{E}+3$ & 42.7
\end{tabular}

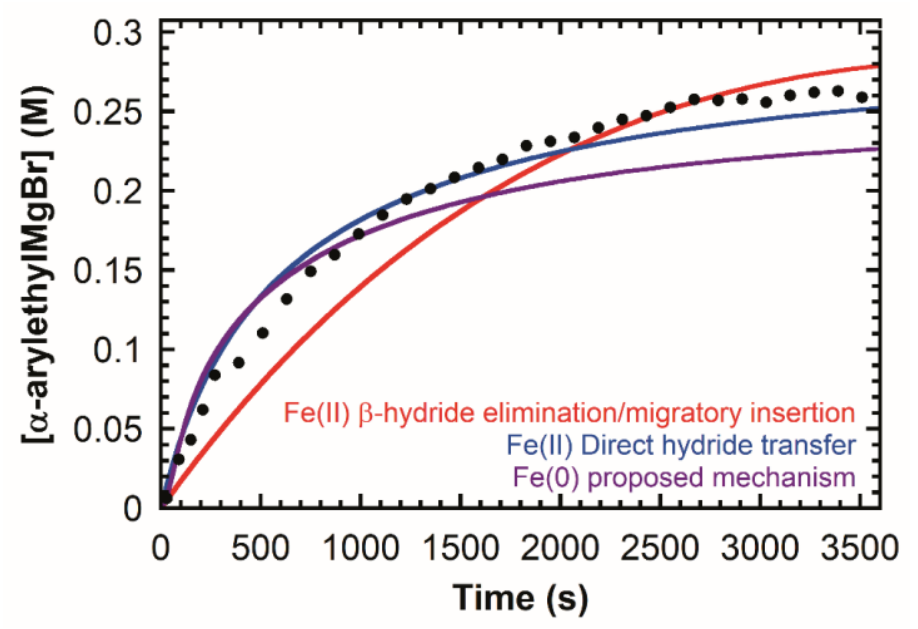

Figure 2.6. Reaction time course and COPASI calculated time courses for mechanisms in Scheme 2.2, [(1-phenylethyl)MgBr] vs time. Conditions: 0.29 M S; 2.91 $\mathrm{mM}\left(\left.\mathrm{PDI}\right|^{\mathrm{iPr}}\right) \mathrm{FeCl}_{2} ; 0.43 \mathrm{M} \mathrm{G} ; 1$ hour; THF; $25^{\circ} \mathrm{C}$. 
To begin the kinetic analysis of hydromagnesiation, the two simplified iron(II)-catalyzed $\beta$-hydride elimination/migratory insertion (BHE/MI) and direct $\beta$-hydride transfer $(\mathrm{DHT})$ mechanisms suggested by Kochi and Thomas were analyzed (Scheme 2.2A). Poor agreement with experimental timecourses were noted for nearly all COPASI estimated timecourses, shown in Figure 2.6 and Tables 2.2-4. The new mechanistic studies which identifies the active catalyst as a formal iron(0)-ate species by Thomas and Neidig also afforded poor agreement computationally (Scheme 2.2B, Figure 2.6). These results were not unexpected given the non-linearity of the catalyst and Grignard initial rates data. A hyperbolic function fit for the catalyst initial rate data (Figure 2.4A) potentially suggests one of two pathways 1) bimolecular decomposition (Scheme $2.5 \mathrm{~A}$ ) or 2) competitive activation. ${ }^{197,198}$ The introduction of an iron decomposition step appeared reasonable based on reported bimolecular reactions of $\left(\mathrm{PDIPr}^{\mathrm{iPr}}\right) \mathrm{FeCl}_{2}$; however, all attempts at bimolecular decomposition resulted in minimal agreement (Table 2.5; Figures 2.7-8; $\mathrm{K}=$ -0.8). ${ }^{199,200}$ Therefore, a competitive activation pathway might explain the hyperbolic fit of the initial rate catalyst analysis. However, more evidence for a competing activation route is needed. The Grignard rate analysis (Figure 2.4C) suggests an apparent saturation behavior based upon a Lineweaver-Burk analysis (Figure 2.9). Addition of a complexation of PDIPr-iron magnesium species also appeared logical by analogy to the organoaluminum complexes with polymerization catalysis, shown in Scheme $2.5 \mathrm{~B}$ insert. ${ }^{76}$ Alas, minimal timecourse agreement was observed in this case as well (Table 2.6; Figures 2.10-11; $\mathrm{K}=$ $-0.60)$. 
A.

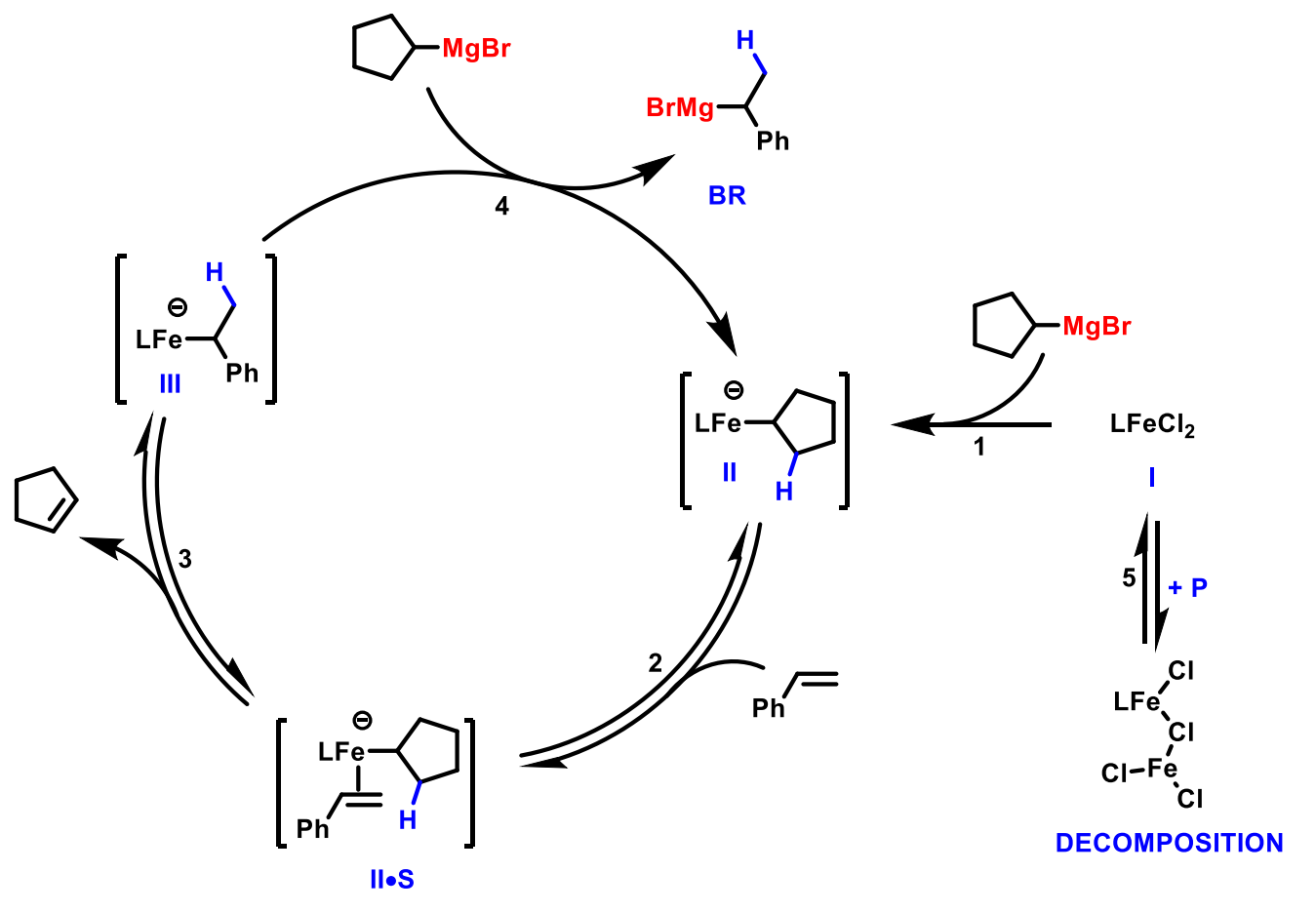

B.

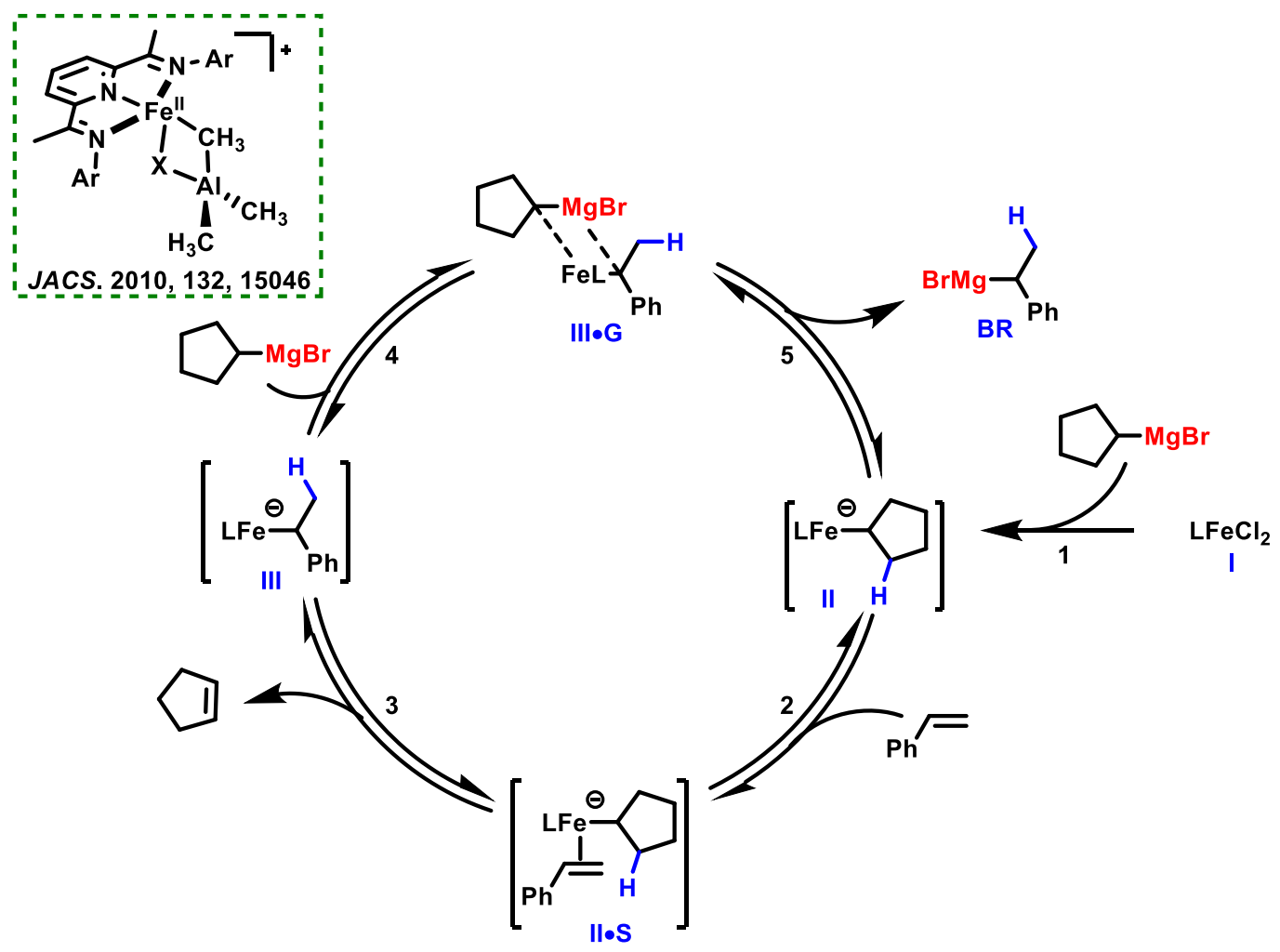

Scheme 2.5: Proposed mechanism introducing A) catalyst decomposition and B) Grignard speciation. 
Table 2.5: Calculated rate constants from COPASI for Scheme 2.5A

\begin{tabular}{l|lll} 
Rxn \# & Conditions & kforward & kreverse \\
\hline 1 & $\mathrm{I}+\mathrm{G}=\mathrm{II}$ & 23.95 & 88.37 \\
2 & $\mathrm{II}+\mathrm{S}=\mathrm{Il} \cdot \mathrm{S}$ & 35.54 & 0.00017 \\
3 & $\mathrm{I} \cdot \mathrm{S}=\mathrm{III}+\mathrm{CYP}$ & 1.43 & 298.44 \\
4 & $\mathrm{III}+\mathrm{G}=\mathrm{BR}+\mathrm{II}$ & 1128.97 & 26247 \\
5 & $\mathrm{I}+\mathrm{I}=\mathrm{DECOMPOSITON}$ & 20.68 & 10557
\end{tabular}

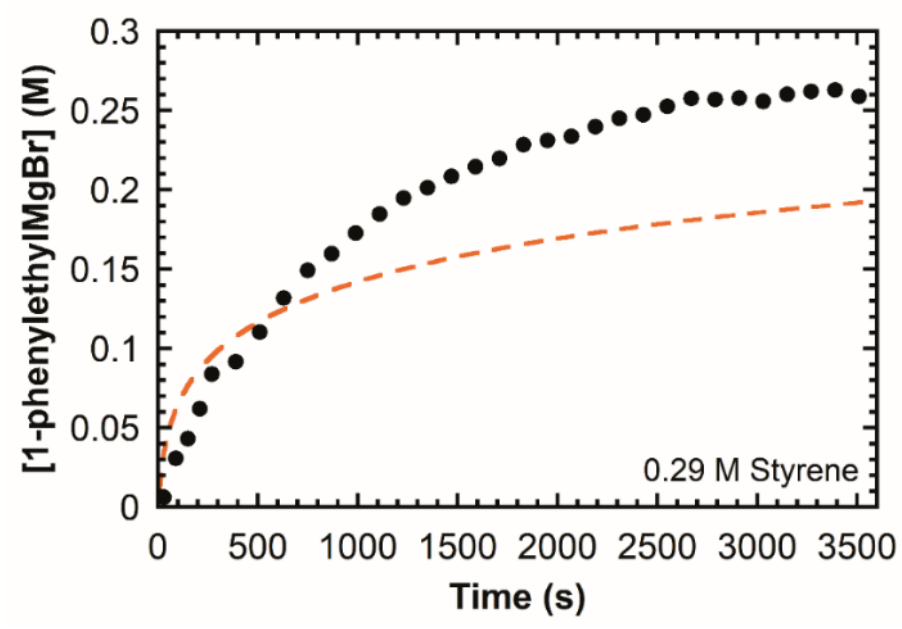

Figure 2.7: Reaction time course and COPASI calculated time courses, [(1phenylethyl) $\mathrm{MgBr}$ ] vs time. Conditions: $0.291 \mathrm{M} \mathrm{S} ; 2.91 \mathrm{mM}\left(\mathrm{PDI}{ }^{\mathrm{PPr}}\right) \mathrm{FeCl}_{2} ; 0.434 \mathrm{M} \mathrm{G}$; 1 hour; THF; $25^{\circ} \mathrm{C}$. 

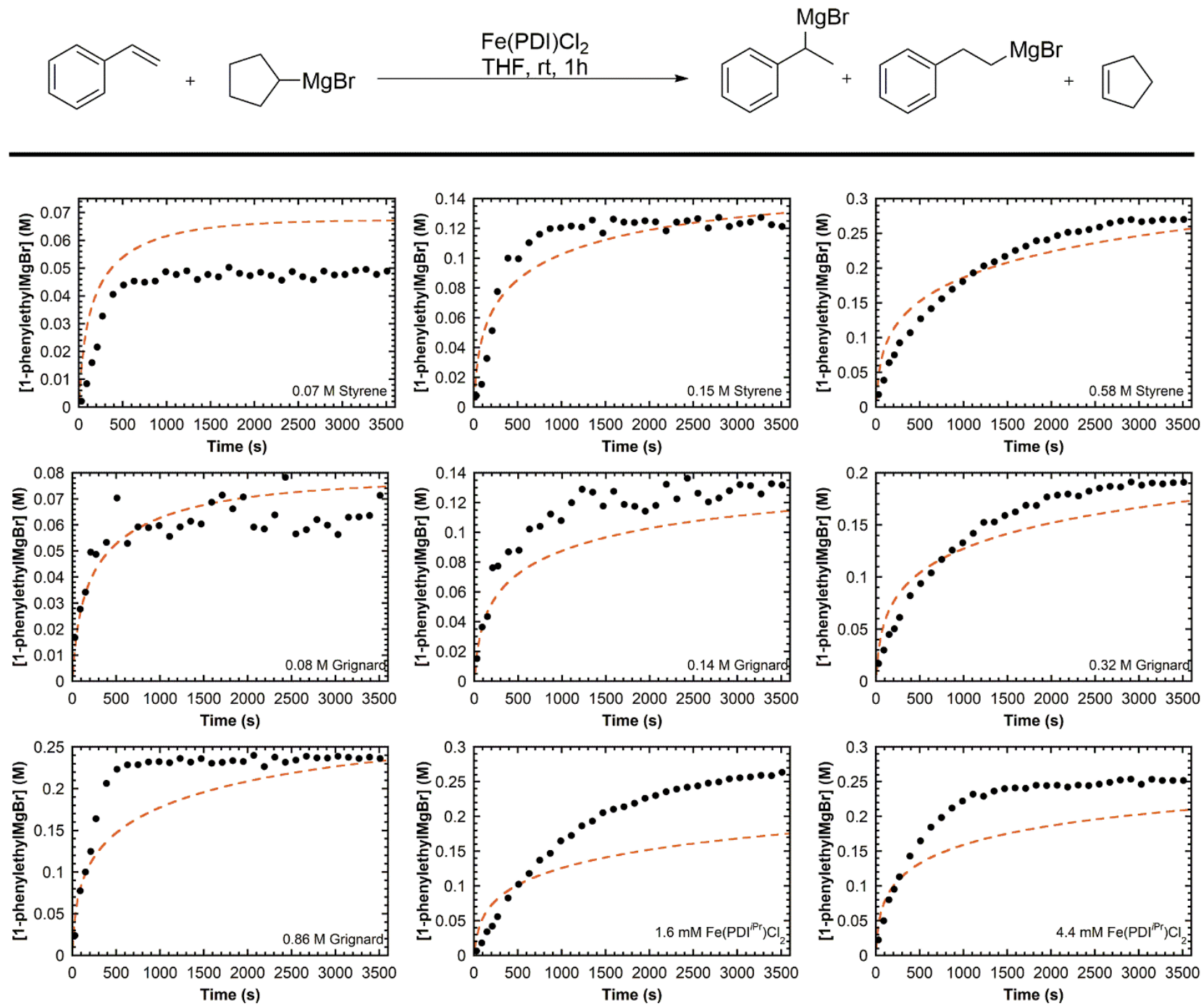

Figure 2.8: Reaction time course and COPASI calculated time courses, [(1phenylethyl) $\mathrm{MgBr}$ vs time. Conditions: $0.291 \mathrm{M} \mathrm{S} ; 2.91 \mathrm{mM}\left(\mathrm{PDI} \mathrm{Pr}^{\mathrm{Pr}} \mathrm{FeCl}_{2} ; 0.434 \mathrm{M} \mathrm{G}\right.$; 1 hour; THF; $25^{\circ} \mathrm{C}$. Varied reaction parameter noted in timecourse plot. Cohen's kappa: minimal agreement $\kappa=\frac{(10 \%)-(50 \%)}{1-50 \%}=-0.8$ 


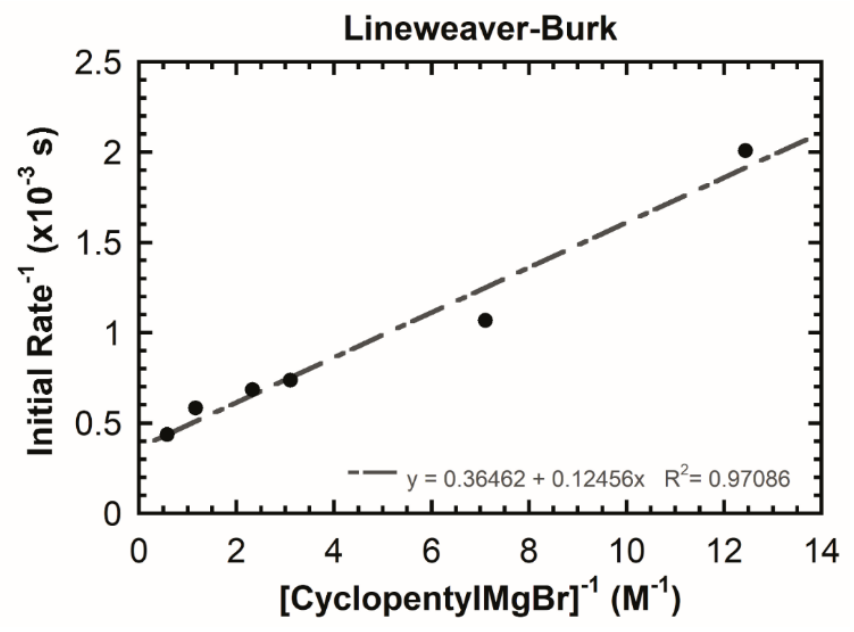

Figure 2.9: Lineweaver-Burk analysis of the Grignard dependence, [(1phenylethyl) $\mathrm{MgBr}$ ] vs time. Conditions: $0.291 \mathrm{M} \mathrm{1;2.9} \mathrm{mM} \mathrm{(PDIPr)FeCl} ;$; $\mathrm{M}$ 2; 1 hour; THF; rt.

Table 2.6: Calculated rate constants from COPASI for Scheme 2.5B

\begin{tabular}{l|lll} 
Rxn \# & Conditions & kforward & kreverse \\
\hline 1 & $\mathrm{I}+\mathrm{G}=\mathrm{II}$ & 8.54 & 9.02 \\
2 & $\mathrm{II}+\mathrm{S}=\mathrm{Il} \cdot \mathrm{S}$ & 241.25 & 0.1299 \\
3 & $\mathrm{II} \cdot \mathrm{S}=\mathrm{III}+\mathrm{CYP}$ & 0.460 & 0.842 \\
4 & $\mathrm{III}+\mathrm{G}=\mathrm{II} \cdot \mathrm{G}$ & 0.233 & 220.42 \\
5 & $\mathrm{II} \cdot \mathrm{G}=\mathrm{BR}+\mathrm{II}$ & 22761.5 & 4020.25
\end{tabular}

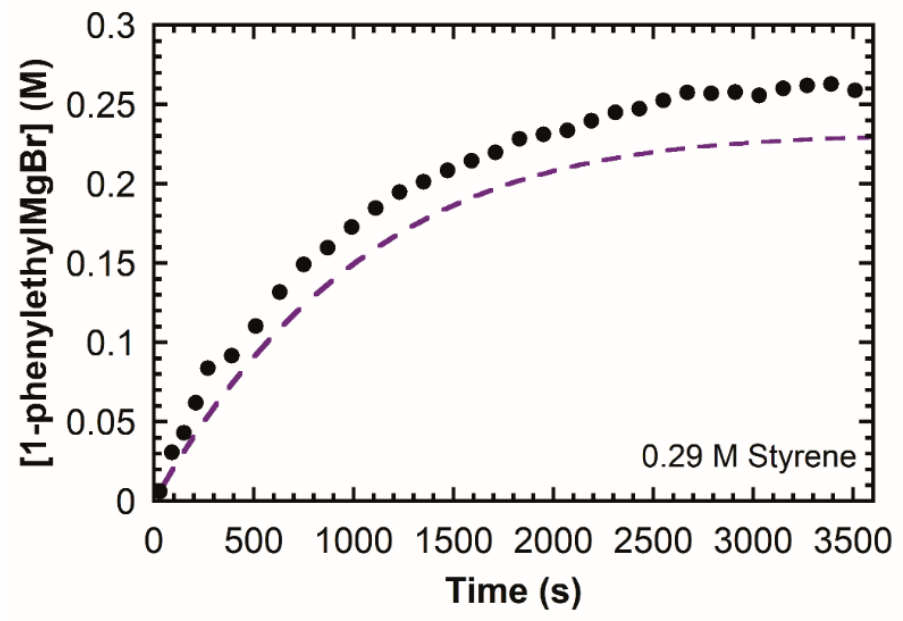

Figure 2.10: Reaction time course and COPASI calculated time courses, [(1phenylethyl) $\mathrm{MgBr}$ ] vs time. Conditions: $0.291 \mathrm{M} \mathrm{S} ; 2.91 \mathrm{mM}\left(\mathrm{PDI}{ }^{\mathrm{PPr}}\right) \mathrm{FeCl}_{2} ; 0.434 \mathrm{M} \mathrm{G}$; 1 hour; THF; $25^{\circ} \mathrm{C}$. 

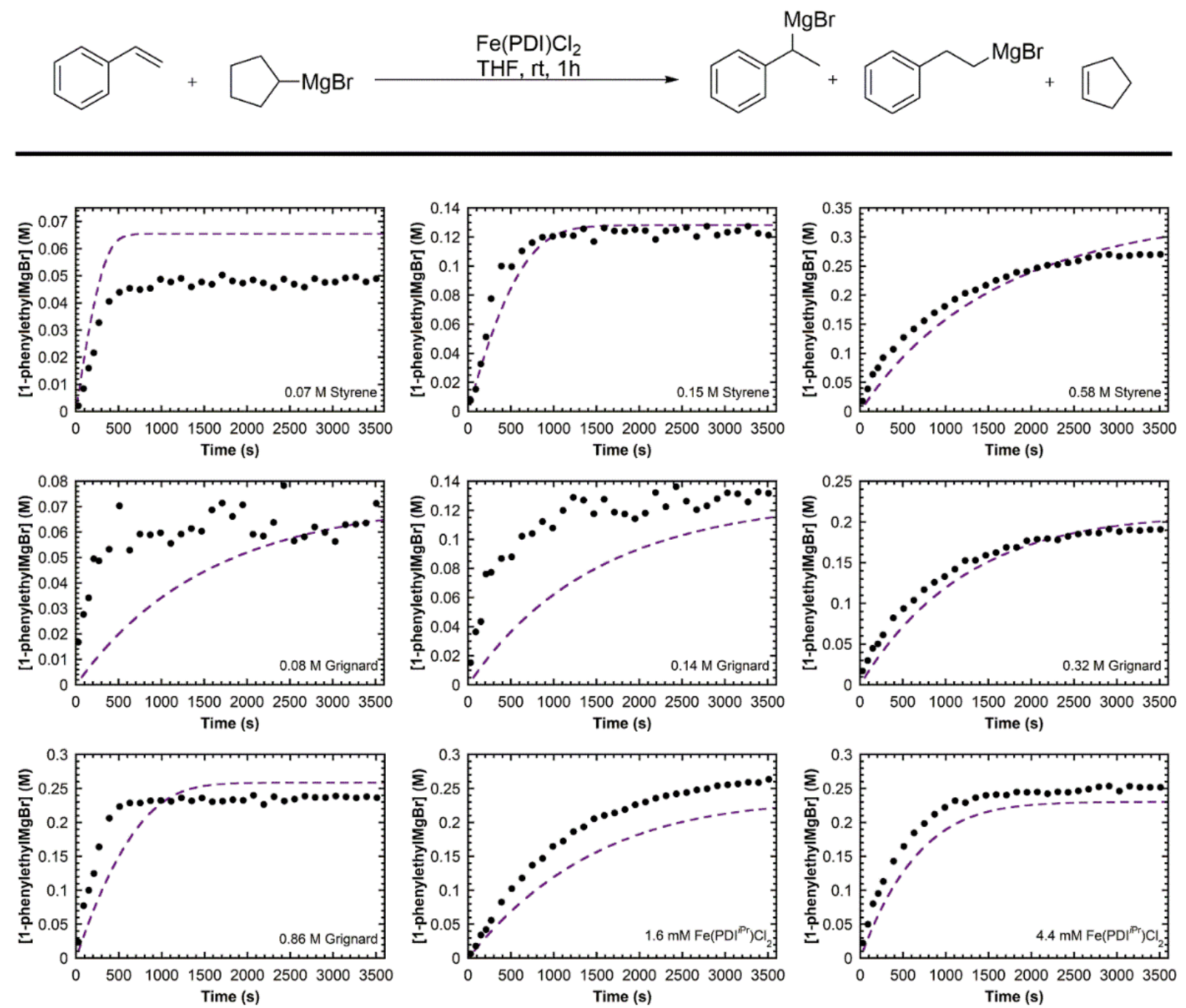

Figure 2.11: Reaction time course and COPASI calculated time courses, [(1phenylethyl)MgBr] vs time. Conditions: $0.291 \mathrm{M} \mathrm{S} ; 2.91 \mathrm{mM}\left(\mathrm{PDI}{ }^{\mathrm{PPr}}\right) \mathrm{FeCl}_{2} ; 0.434 \mathrm{M} \mathrm{G}$; 1 hour; THF; $25^{\circ} \mathrm{C}$. Varied reaction parameter noted in timecourse plot. Cohen's kappa: minimal agreement $\kappa=\frac{(20 \%)-(50 \%)}{1-50 \%}=-0.6$

Elementary steps were introduced to assess the mechanistic impact of three complex kinetic observations: 1) linear initial rate dependence with a non-zero intercept for $S$ (Figure 2.4E); 2) inhibitory behavior for both $S$ and $\mathrm{G}^{\mathrm{CYP}}$ at low concentrations; 3 ) varying amounts of Grignard product, L, depending on reaction conditions. First, the non-zero intercept first order behavior of $S$ suggests the possibility of a styrene-promoted catalyst activation, shown in Scheme 2.6A (Steps 1A-2A). This styrene-promoted catalyst activation in this case, based upon COPASI calculated rate constants, outcompetes the 
unaided Grignard activation (Steps 1-2). In enzymatic kinetics, this behavior is well known and results in hyperbolic catalyst dependencies, thus alludes to the explanation of the experimentally determined catalyst hyperbolic initial rates regime. ${ }^{201}$ The mechanism in Scheme 2.6A, though a minimal increase in agreement was observed (Table 2.7; Figure 2.12-13; $\mathrm{k}=-0.6)$, did not account for the formation of the two regioisomers, branched product $(B R)$ and linear product $(L)$, nor did it justify the saturation dependence of $G$ CYP. Scheme 2.6B aims to rationalize both instances. Introduction of steps 2 and 2B in Scheme 2.6B increased the agreement from -0.6 to -0.4 (Table 2.8; Figures 2.14-15); therefore, the complexities noted by the observed rates in Figure 2.4D,F need to be accounted for.

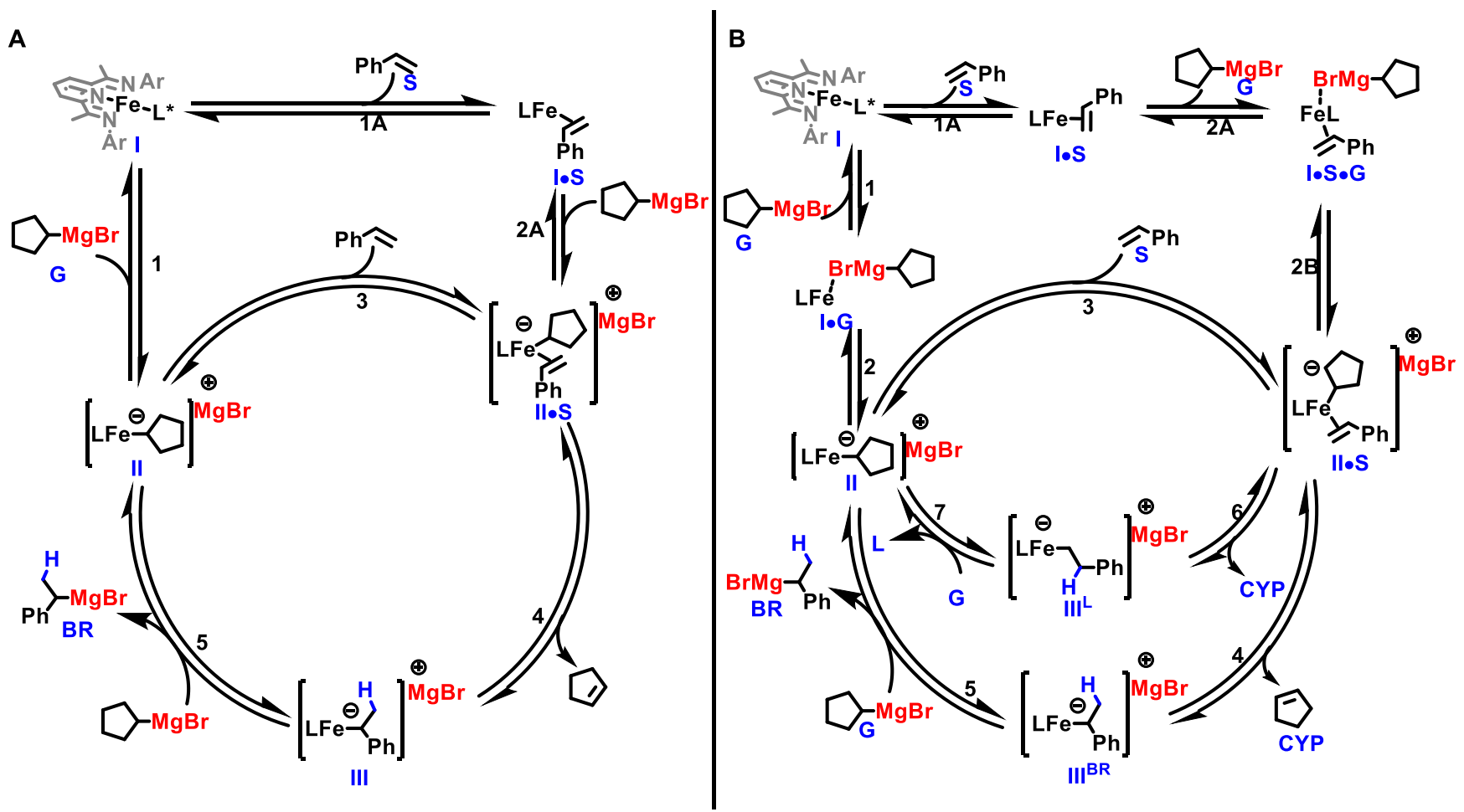

Scheme 2.6: A) Proposed mechanism introducing a styrene catalyzed activation, B) Proposed mechanism introducing a 2-step styrene catalyzed activation. $\left(L=P D I^{i P r}, L\right.$ = linear isomer) 
Table 2.7: Calculated rate constants from COPASI for Scheme 2.6A

\begin{tabular}{l|lll} 
Rxn \# & Conditions & kforward & kreverse \\
\hline $1 A$ & $I+S=I \cdot S$ & 0.014 & $6.15 \times 10^{-6}$ \\
$2 A$ & $\cdot \cdot S+G=\| \cdot S$ & 2770.2 & $1.0 \times 10^{-6}$ \\
1 & $I+G=\|$ & 48.65 & $1.0 \times 10^{-6}$ \\
3 & $I I+S=\| \cdot S$ & 859.28 & 15.39 \\
4 & $\| \cdot S=I I I+C Y P$ & 19.75 & 1022.8 \\
5 & $I I I+G=B R+I I$ & 1.026 & $2.29 \times 10^{-5}$
\end{tabular}

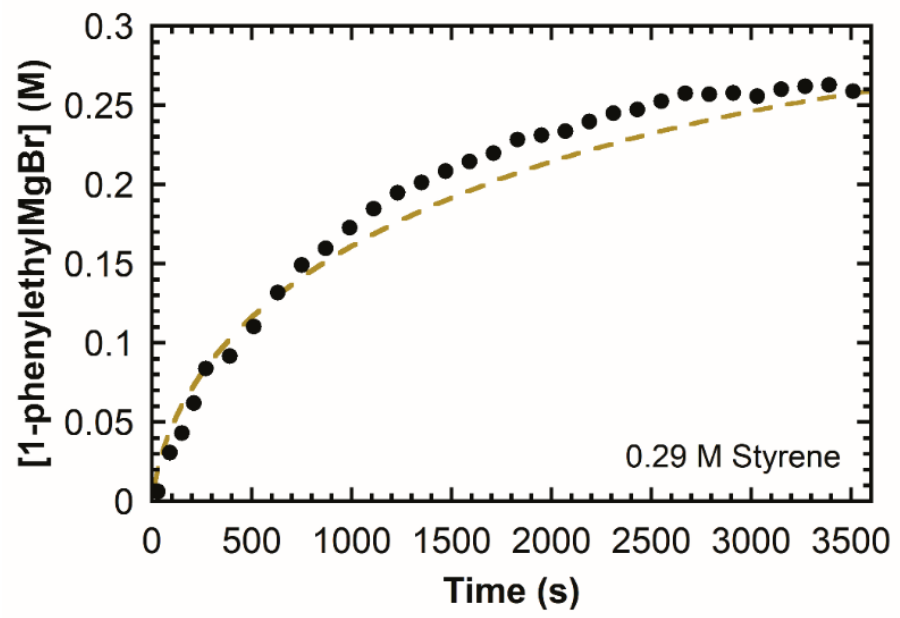

Figure 2.12: Reaction time course and COPASI calculated time courses, [(1phenylethyl) $\mathrm{MgBr}$ ] vs time. Conditions: $0.291 \mathrm{M} \mathrm{S} ; 2.91 \mathrm{mM}\left(\mathrm{PDl}{ }^{\mathrm{PPr}}\right) \mathrm{FeCl}_{2} ; 0.434 \mathrm{M} \mathrm{G}$; 1 hour; THF; $25^{\circ} \mathrm{C}$. 

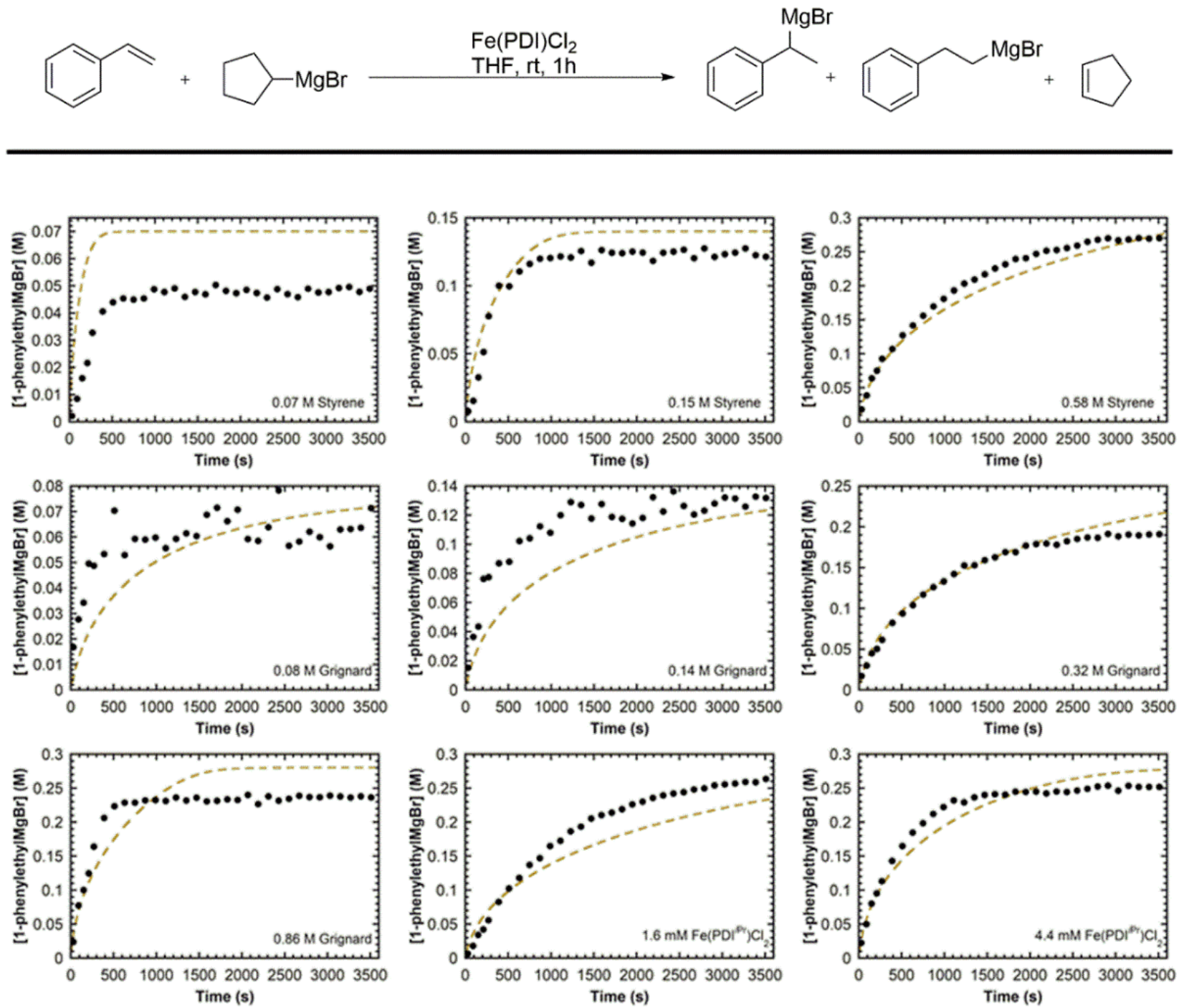

Figure 2.13: Reaction time course and COPASI calculated time courses, [(1phenylethyl) $\mathrm{MgBr}$ ] vs time. Conditions: $0.291 \mathrm{M} \mathrm{S} ; 2.91 \mathrm{mM}\left(\mathrm{PDI} \mathrm{Pr}^{\mathrm{PP}} \mathrm{FeCl}_{2} ; 0.434 \mathrm{M} \mathrm{G}\right.$; 1 hour; THF; $25{ }^{\circ} \mathrm{C}$. Varied reaction parameter noted in timecourse plot. Cohen's kappa: minimal agreement $\kappa=\frac{(20 \%)-(50 \%)}{1-50 \%}=-0.6$ 
Table 2.8: Calculated rate constants from COPASI for Scheme 2.6B

\begin{tabular}{l|lll} 
Rxn \# & Conditions & kforward & kreverse \\
\hline $1 \mathrm{~A}$ & $\mathrm{I}+\mathrm{S}=\mathrm{I} \cdot \mathrm{S}$ & 6246.05 & 1.372 \\
$2 \mathrm{~A}$ & $\mathrm{I} \cdot \mathrm{S}+\mathrm{G}=\mathrm{I} \cdot \mathrm{S} \cdot \mathrm{G}$ & 134.87 & 14.86 \\
$2 \mathrm{~B}$ & $\mathrm{I} \cdot \mathrm{S} \cdot \mathrm{G}=\| \cdot \mathrm{I}$ & 0.0224 & 0.0731 \\
1 & $\mathrm{I}+\mathrm{G}=\mid \cdot \mathrm{G}$ & 0.000315 & 0.000814 \\
2 & $\mathrm{I} \cdot \mathrm{G}=\mathrm{II}$ & 36697.3 & 69.89 \\
3 & $\mathrm{II}+\mathrm{S}=\| \cdot \mathrm{I}$ & 21.99 & 0.00439 \\
4 & $\mathrm{II} \cdot \mathrm{S}=\mathrm{IIIBR}+\mathrm{CYP}$ & 2.087 & 12018.3 \\
5 & $\mathrm{IIIBR}+\mathrm{G}=\mathrm{BR}+\mathrm{II}$ & 3734.54 & 0.221 \\
6 & $\mathrm{II} \cdot \mathrm{S}=\mathrm{III}+\mathrm{CYP}$ & 0.116 & $8.09 \times 10^{-6}$ \\
7 & $\mathrm{III}+\mathrm{G}=\mathrm{II}+\mathrm{L}$ & 0.0333 & 0.000258
\end{tabular}

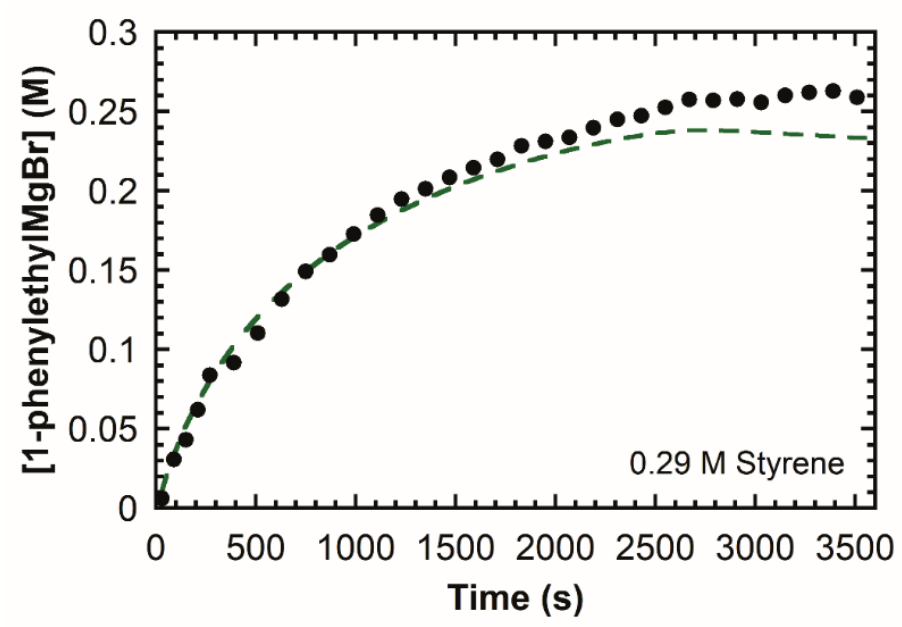

Figure 2.14: Reaction time course and COPASI calculated time courses, [(1phenylethyl)MgBr] vs time. Conditions: $0.291 \mathrm{M} \mathrm{S} ; 2.91 \mathrm{mM}\left(\left.\mathrm{PDI}\right|^{\mathrm{iPr}}\right) \mathrm{FeCl}_{2} ; 0.434 \mathrm{M} \mathrm{G}$; 1 hour; THF; $25^{\circ} \mathrm{C}$. 

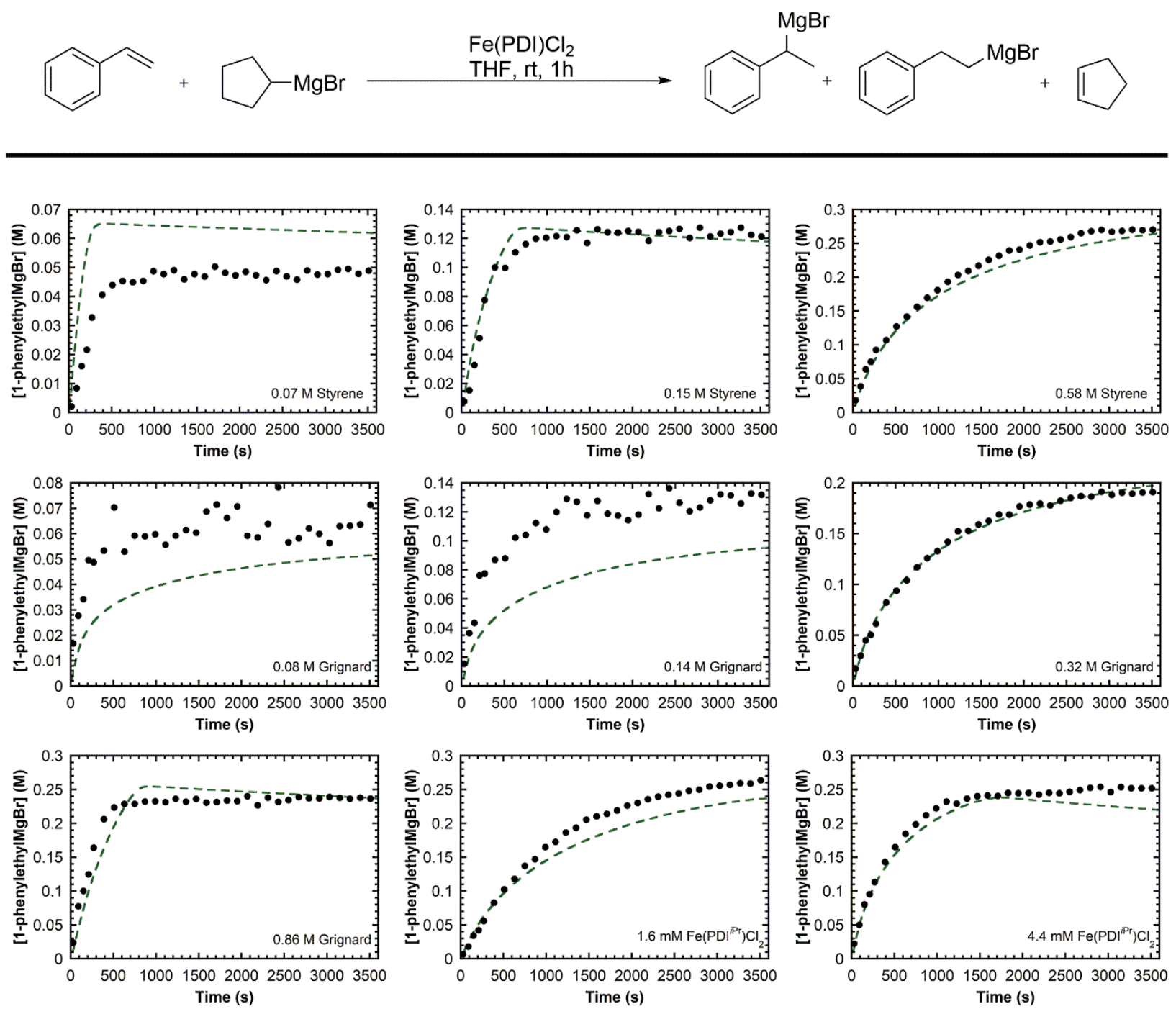

Figure 2.15: Reaction time course and COPASI calculated time courses, [(1phenylethyl)MgBr] vs time. Conditions: $0.291 \mathrm{M} \mathrm{S} ; 2.91 \mathrm{mM}\left(\mathrm{PDI}{ }^{\mathrm{PPr}}\right) \mathrm{FeCl}_{2} ; 0.434 \mathrm{M} \mathrm{G}$; 1 hour; THF; $25^{\circ} \mathrm{C}$. Varied reaction parameter noted in timecourse plot. Cohen's kappa: minimal agreement $\kappa=\frac{(60 \%)-(50 \%)}{1-50 \%}=-0.4$

To explain the inhibitory behavior of styrene at low concentrations, a reversible pathway by which iron-benzyl, IIIRR, may be trapped off-cycle preceding the TLS step, transmetallation (step 5), was introduced (Scheme 2.7, Step 8). The resulting COPASI timecourse analysis, with all elementary steps set as reversible, yielded excellent agreement over the entire timecourse collection (Scheme 2.7; Figure 2.16-17; and $\mathrm{k}=$ 0.80). Significantly, all attempts to identify a mechanism in which a distinct $\mathrm{Fe}-\mathrm{H}$ intermediate (BHE/MI pathway described initially by Kochi) was kinetically relevant were 
unsuccessful, supporting Thomas' proposal that this hydromagnesiation proceeds through a DHT pathway. Although the means of hydride transfer favor DHT over BHE/MI, experimental evidence for DHT over BHE/MI by initial rates/observed rates analyses is still considered to be kinetically transparent.

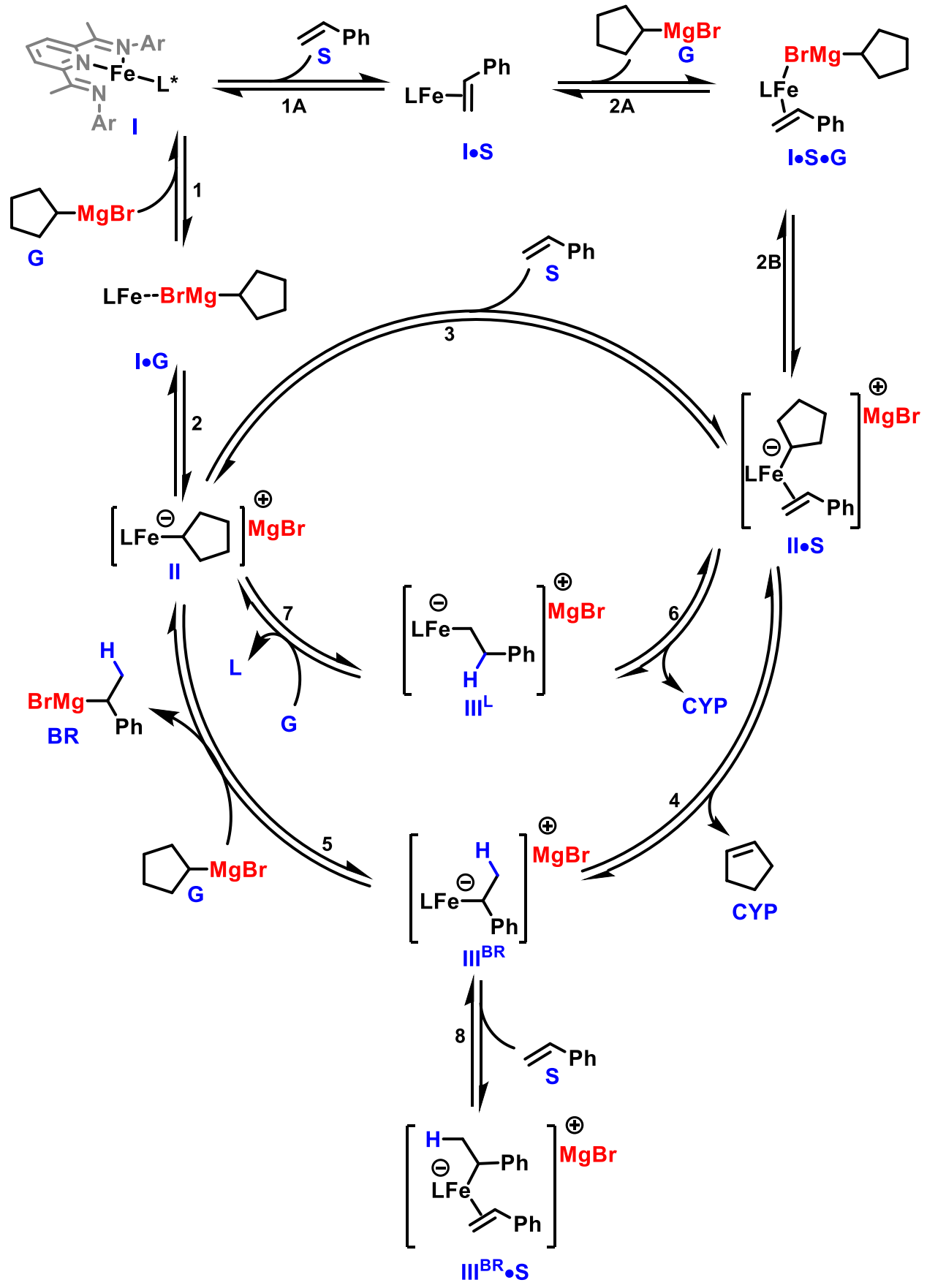

Scheme 2.7: Proposed mechanism introducing a catalyst decomposition pathway by styrene. $(\mathrm{L}=\mathrm{PDI} \mathrm{PPr}, \mathrm{L}=$ linear isomer) 
Table 2.9: Calculated rate constants from COPASI for Scheme 2.7

\begin{tabular}{|c|c|c|c|}
\hline Rxn \# & Conditions & k.forward & k.reverse \\
\hline $1 \mathrm{~A}$ & $I+S=1 \cdot S$ & 223087.0 & 0.262 \\
\hline $2 \mathrm{~A}$ & $1 \cdot S+G=1 \cdot S \cdot G$ & 16810.4 & 0.126 \\
\hline $2 B$ & $\mid \cdot S \cdot G=\| \cdot S$ & 41459.4 & 0.00116 \\
\hline 1 & $I+G=1 \cdot G$ & 1.13 & 904.08 \\
\hline 2 & $\mid \cdot G=\|$ & 46.24 & 0.086 \\
\hline 3 & $\|+S=\| \cdot S$ & 12.062 & 0.060 \\
\hline 4 & $\| \cdot S=I I I B R+C Y P$ & 0.32 & 0.011 \\
\hline 5 & $\mathrm{IIIBR}+\mathrm{G}=\mathrm{BR}+\mathrm{II}$ & 0.41 & 2.88 \\
\hline 6 & $\| \cdot S=I I L+C Y P$ & 0.069 & 0.40 \\
\hline 7 & $I I I L+G=I I+L$ & 2.62 & 5.74 \\
\hline 8 & $\mathrm{IIIBR}+\mathrm{S}=\mathrm{IIIBR} \cdot \mathrm{S}$ & 0.0016 & $8.85 \times 10-5$ \\
\hline
\end{tabular}

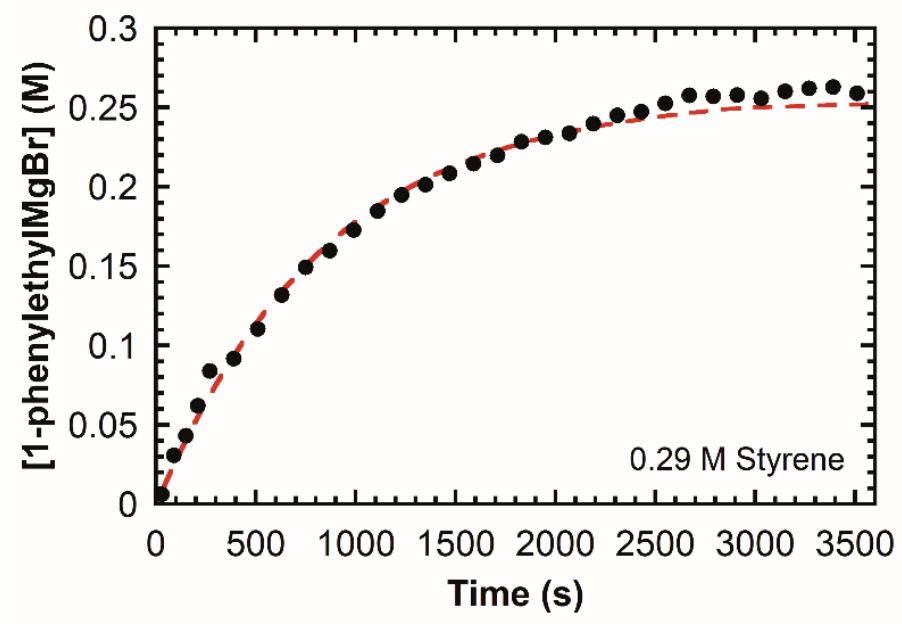

Figure 2.16: Reaction time course and COPASI calculated time courses, [(1phenylethyl) $\mathrm{MgBr}$ ] vs time. Conditions: $0.291 \mathrm{M} \mathrm{S} ; 2.91 \mathrm{mM}\left(\mathrm{PDI} \mathrm{Pr}^{\mathrm{PP}}\right) \mathrm{FeCl}_{2} ; 0.434 \mathrm{M} \mathrm{G}$; 1 hour; THF; $25^{\circ} \mathrm{C}$. 

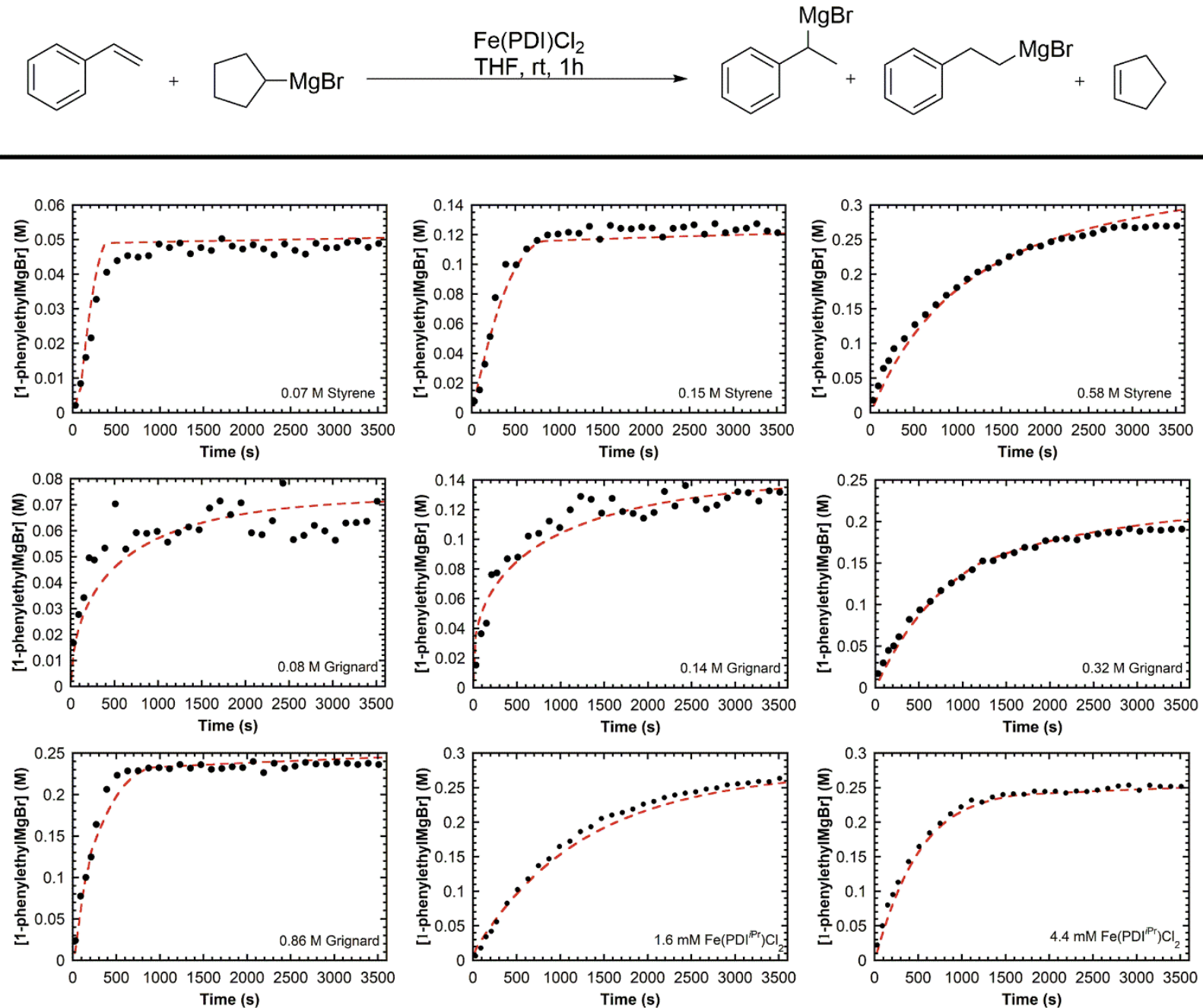

Figure 2.17: Reaction time course from and COPASI calculated time courses, [(1phenylethyl) $\mathrm{MgBr}$ ] vs time. Conditions: $0.291 \mathrm{M} \mathrm{S} ; 2.91 \mathrm{mM}\left(\mathrm{PDI} \mathrm{Pr}^{\mathrm{PP}} \mathrm{FeCl}_{2} ; 0.434 \mathrm{M} \mathrm{G}\right.$; 1 hour; THF; $25{ }^{\circ} \mathrm{C}$. Varied reaction parameter noted in timecourse plot. Cohen's kappa: excellent agreement $\kappa=\frac{(90 \%)-(50 \%)}{1-50 \%}=0.80$

Thomas noted that linear Grignard, L, isomerized to branched Grignard, BR, in the presence of an alkene and iron catalyst. ${ }^{153,170,195}$ The isomerization reaction in the presence of styrene (10 mol\% and $100 \mathrm{~mol} \%$ ) was monitored by in situ IR spectroscopy and the observed rate of isomerization was calculated to be only a single order of magnitude slower than the initial rate of hydromagnesiation (Figure 2.18), implying that the isomerization process is kinetically relevant under certain reaction regimes. A direct isomerization path from IIIL to III ${ }^{\mathrm{BR}}$ was considered, Scheme 2.8. Upon introduction of this 
new step, shown in Scheme 2.8 and Step 9, poorer agreement was observed when elementary steps 1-8 were set as reversible. Recognizing that previous mechanistic work with $\mathrm{G}^{\mathrm{Et}}$ led to the conclusion that formation of both intermediates $\mathrm{III}^{\mathrm{BR}}$ and III , and product BR were irreversible, steps 4-6 were also evaluated as irreversible within the numeric model with COPASI. The resulting kinetic model exhibited excellent agreement $(\mathrm{k}=0.84)$ over all timecourses collected (an assortment of 24 data sets), see Figures 2.19-21. The irreversibility of Step 9 is also connected to both the findings of isomerization reported in Figure 2.18 and previous deuterium studies discussed by Thomas, Greenhalgh, and Neidig, wherein BR does not reenter the catalytic cycle (Scheme 2.3B). ${ }^{153,170,195}$ The kinetic model was then used to predict, with good agreement, timecourse data for a reaction in which regioselectivity is initially poor but improves steadily over time as observed previously by Thomas (Figure 2.21). ${ }^{153,170,195}$
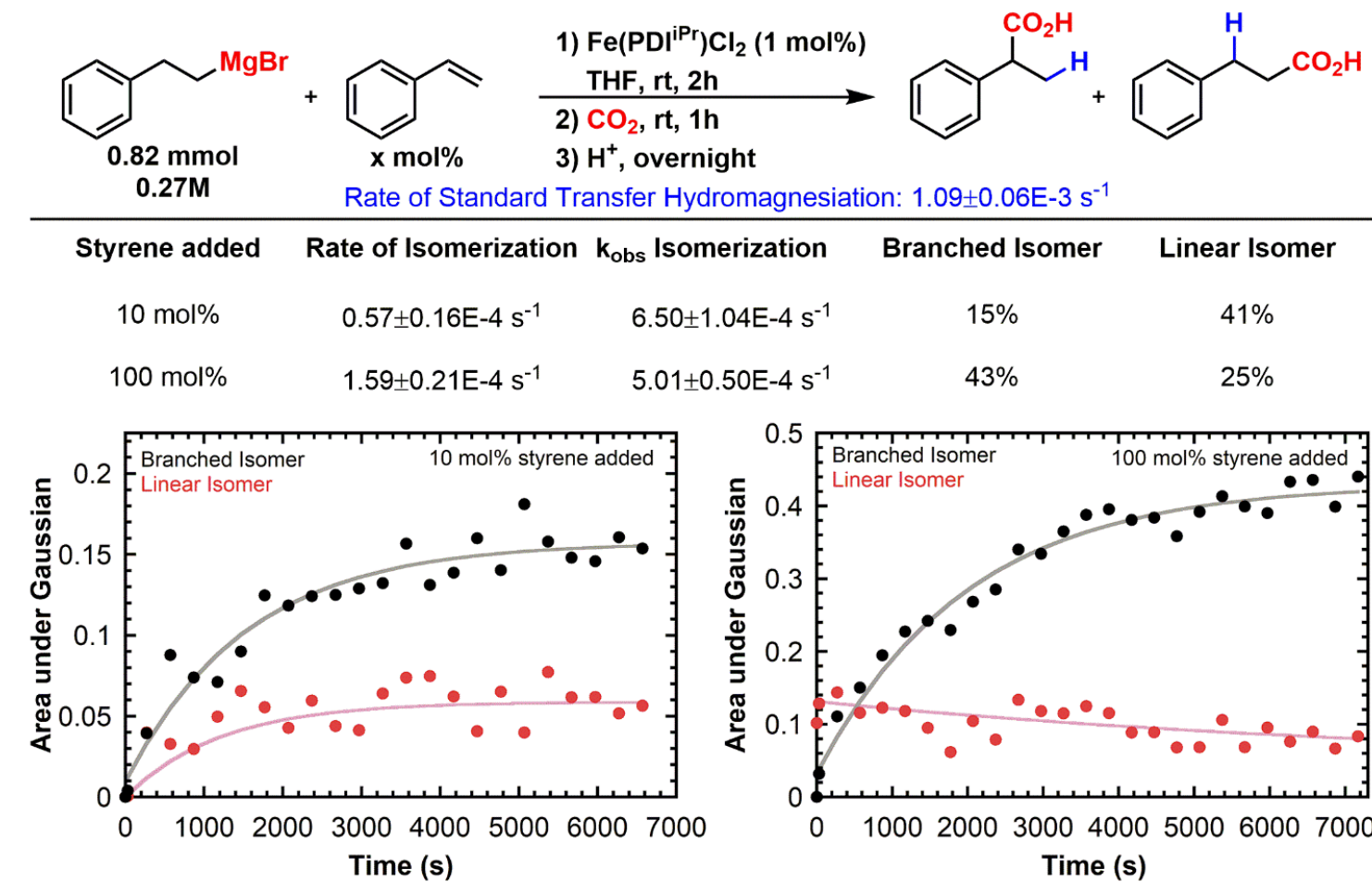

Branched Isomer

Linear Isomer

$\begin{array}{lllll}10 \mathrm{~mol} \% & 0.57 \pm 0.16 \mathrm{E}-4 \mathrm{~s} \mathrm{~s}^{-1} & 6.50 \pm 1.04 \mathrm{E}-4 \mathrm{~s}^{-1} & 15 \% & 41 \% \\ 100 \mathrm{~mol} \% & 1.59 \pm 0.21 \mathrm{E}-4 \mathrm{~s} \mathrm{~s}^{-1} & 5.01 \pm 0.50 \mathrm{E}-4 \mathrm{~s}^{-1} & 43 \% & 25 \%\end{array}$

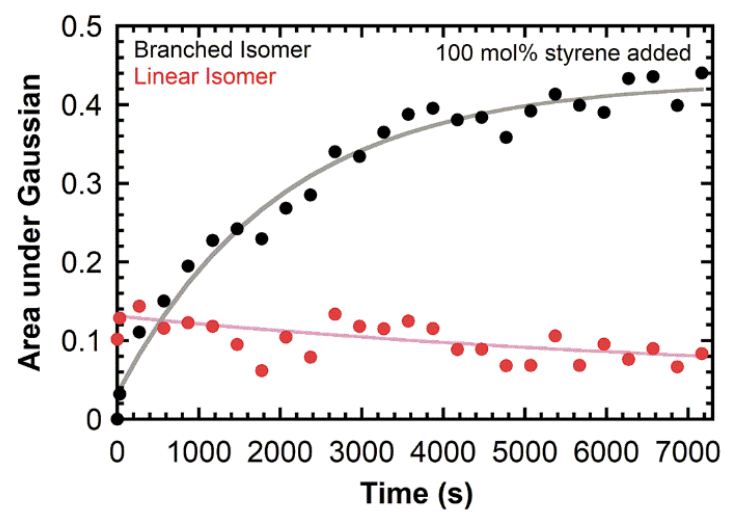

Figure 2.18: Isomerization of linear Grignard to branched followed by carboxylation, Area of Gaussian vs time. Conditions: $0.027 \mathrm{M}, 0.27 \mathrm{M} \mathrm{S} ; 2.9 \mathrm{mM}$ (PDI iPr)FeCl2; 0.27 M L; 1 hour; THF; rt. 


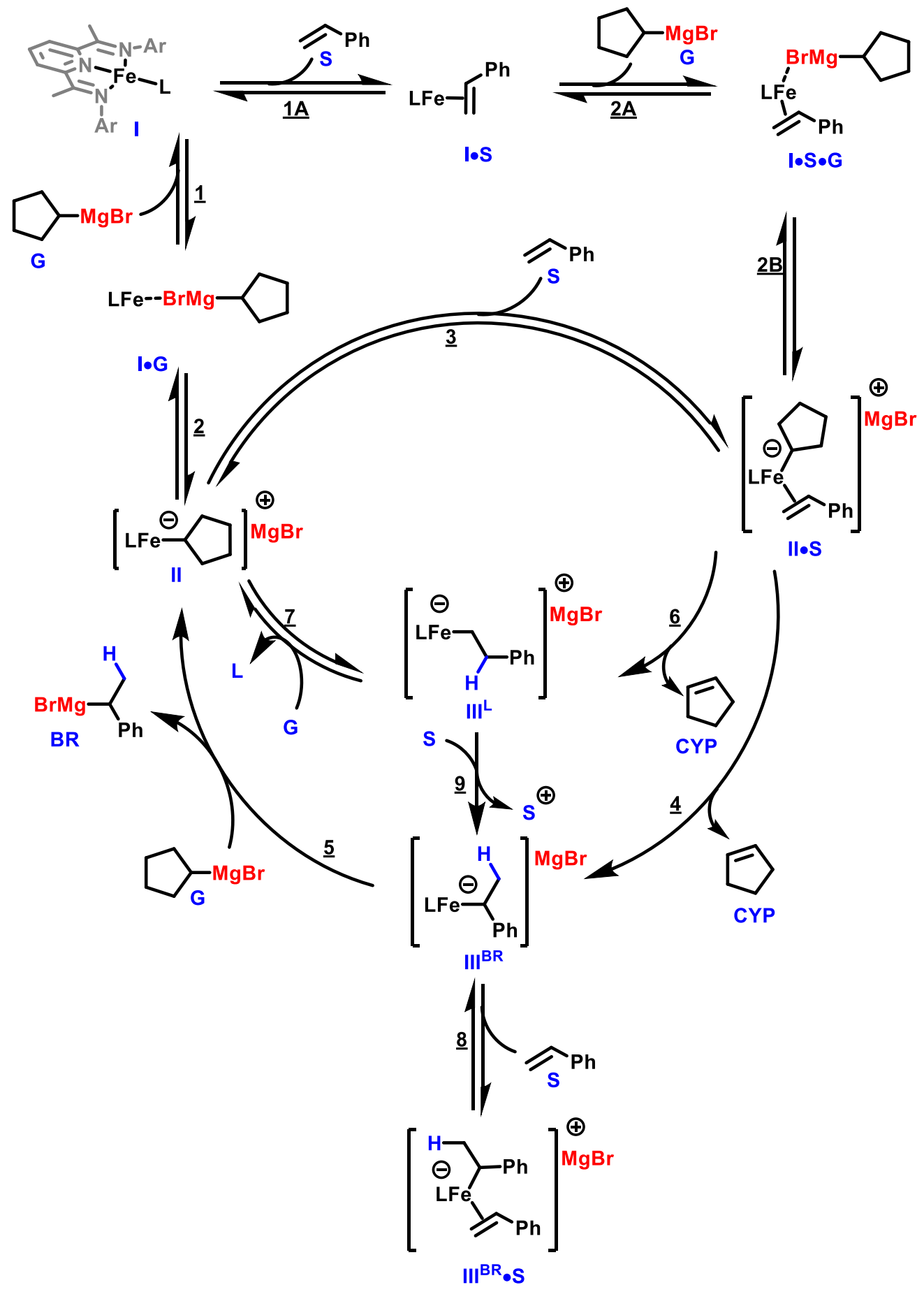

Scheme 2.8: Proposed mechanism of iron-catalyzed hydromagnesiation. ( $L=P D I^{i P r}$, $\mathrm{L}=$ linear isomer) 
Table 2.10: Calculated rate constants from COPASI for Scheme 2.8

\begin{tabular}{|c|c|c|c|}
\hline Rxn \# & Conditions & k.forward & k.reverse \\
\hline $1 \mathrm{~A}$ & $I+S=1 \cdot S$ & 39990 & 1.76 \\
\hline $2 \mathrm{~A}$ & $1 \cdot S+G=1 \cdot S \cdot G$ & 262.64 & 0.083 \\
\hline 2B & $\mid \cdot S \cdot G=\| \cdot S$ & 54551.6 & $1.28 \times 10^{-4}$ \\
\hline 1 & $1+G=1 \cdot G$ & 5.83 & 4421.84 \\
\hline 2 & $\mid \cdot G=\|$ & 68.71 & 0.53 \\
\hline 3 & $\|+S=\| \cdot S$ & 1.37 & 0.29 \\
\hline 4 & $\|\cdot S->\| \|^{B R}+C Y P$ & 1.35 & --- \\
\hline 5 & $\mathrm{III}^{\mathrm{BR}}+\mathrm{G}->\mathrm{BR}+\mathrm{II}$ & 1.41 & --- \\
\hline 6 & $\| \cdot S->I I^{L}+C Y P$ & 0.22 & -- \\
\hline 7 & $\left\|I^{L}+G=\right\|+L$ & 0.39 & 43.74 \\
\hline 8 & $\left\|I^{B R}+S->\right\| I^{B R} \cdot S$ & 0.0072 & $2.03 \times 10^{-4}$ \\
\hline 9 & $\| I I^{L}+S=I I^{B R}+S$ & 0.0084 & --- \\
\hline
\end{tabular}

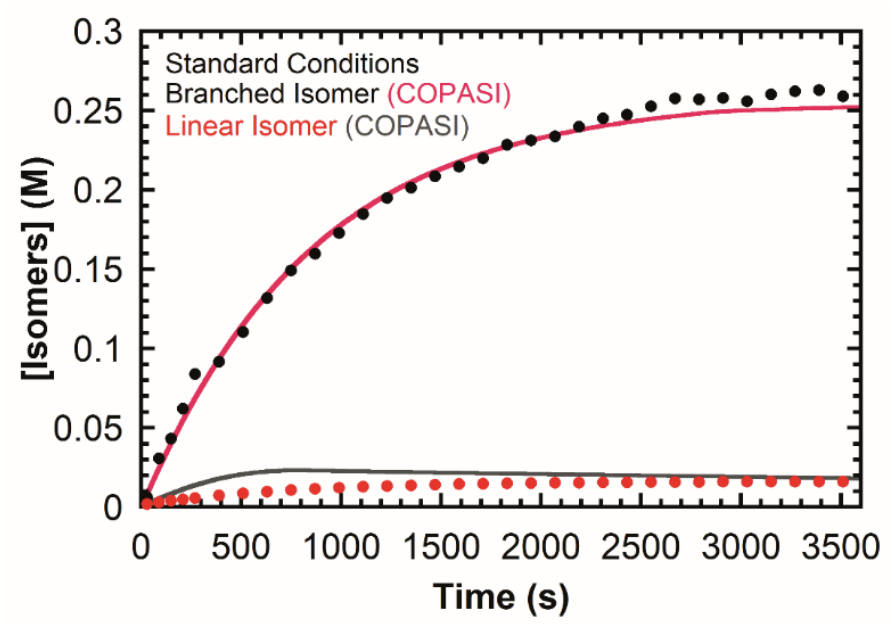

Figure 2.19: Reaction time course from and COPASI calculated time courses, [(1phenylethyl) $\mathrm{MgBr}]$ and [(2-phenylethyl) $\mathrm{MgBr}]$ vs time. Conditions: $0.291 \mathrm{M} \mathrm{S} ; 2.91$ $\mathrm{mM}\left(\mathrm{PDI}{ }^{\mathrm{PPr}}\right) \mathrm{FeCl}_{2} ; 0.434 \mathrm{M} \mathrm{G} ; 1$ hour; THF; $25^{\circ} \mathrm{C}$. 

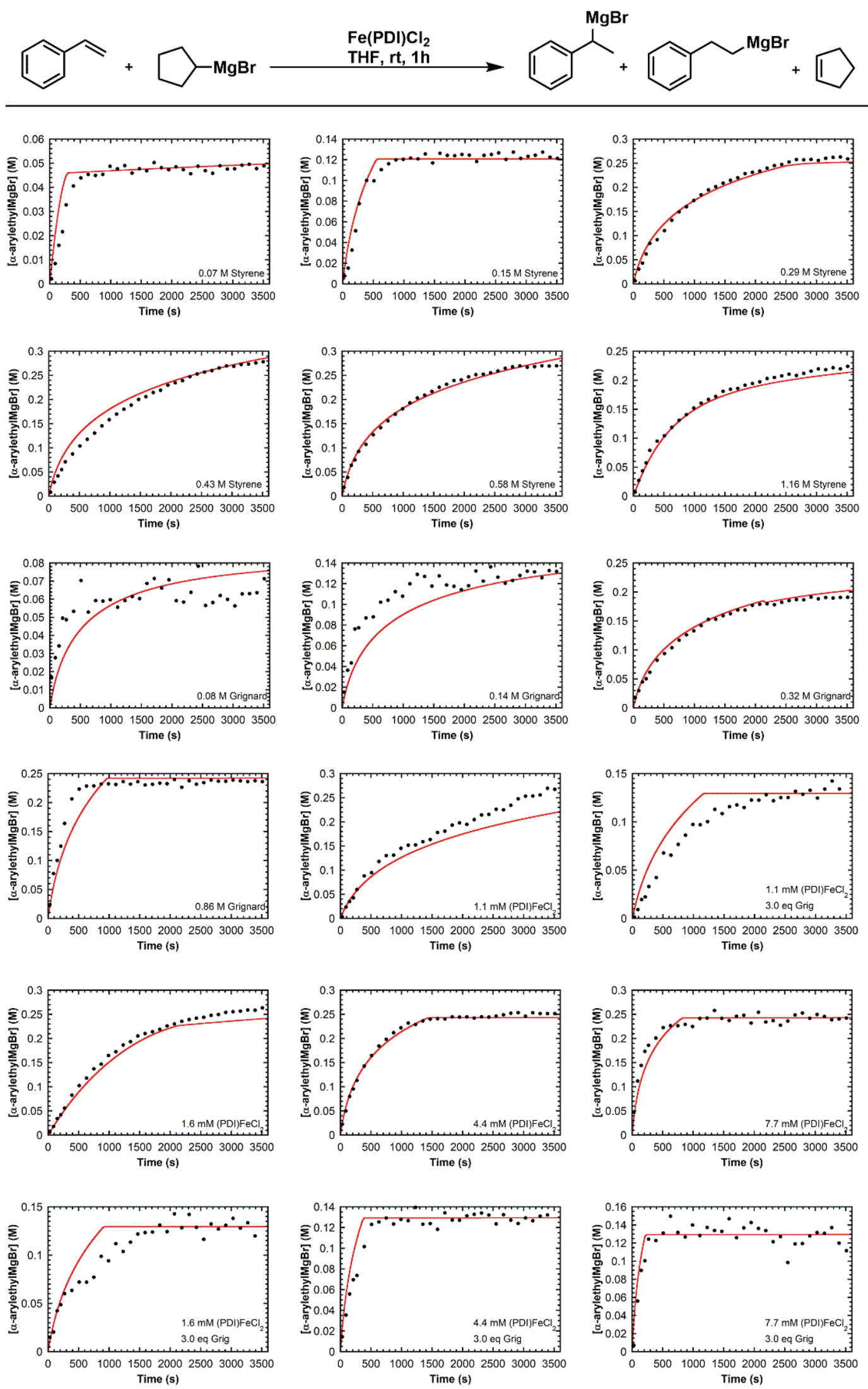

note: Figure 2.20 continued onto page 54 $-53-$ 

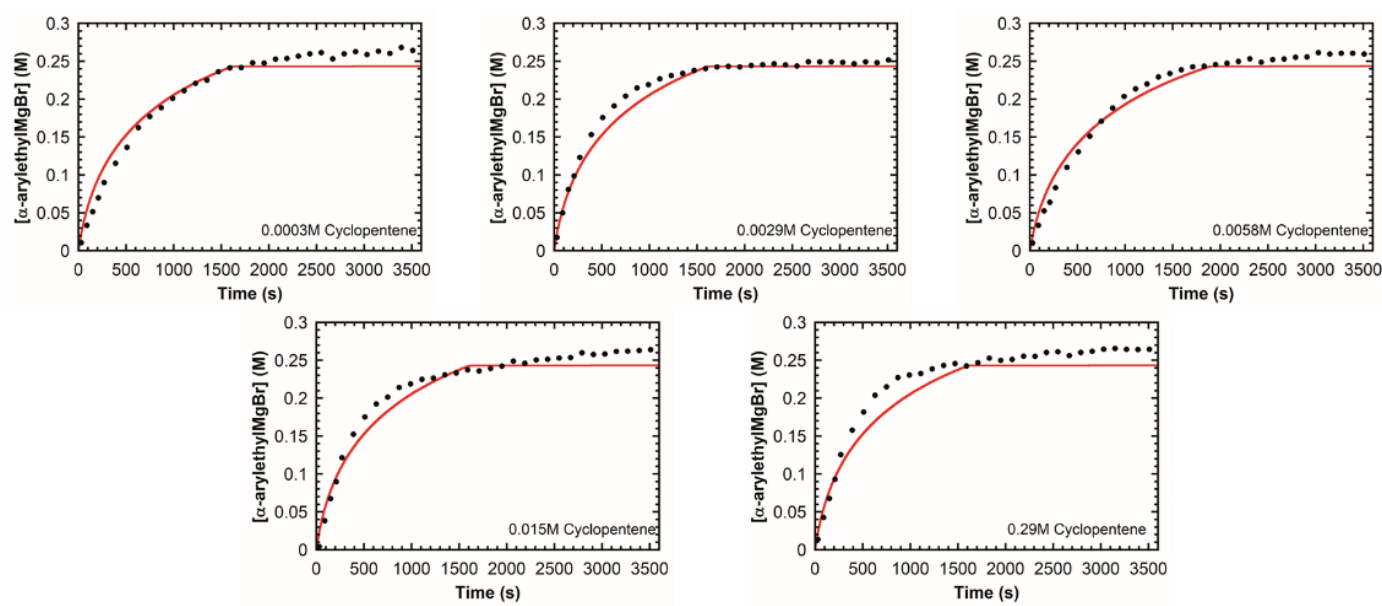

Figure 2.20 Reaction time course from and COPASI calculated time courses, [(1phenylethyl)MgBr] vs time. Conditions: $0.291 \mathrm{M} \mathrm{S} ; 2.91 \mathrm{mM}\left(\mathrm{PDI}{ }^{\mathrm{PPr}}\right) \mathrm{FeCl}_{2} ; 0.434 \mathrm{M} \mathrm{G}$; 1 hour; THF; $25{ }^{\circ} \mathrm{C}$. Varied reaction parameter noted in timecourse plot. Cohen's kappa: excellent agreement $\kappa=\frac{(92 \%)-(50 \%)}{1-50 \%}=0.84$

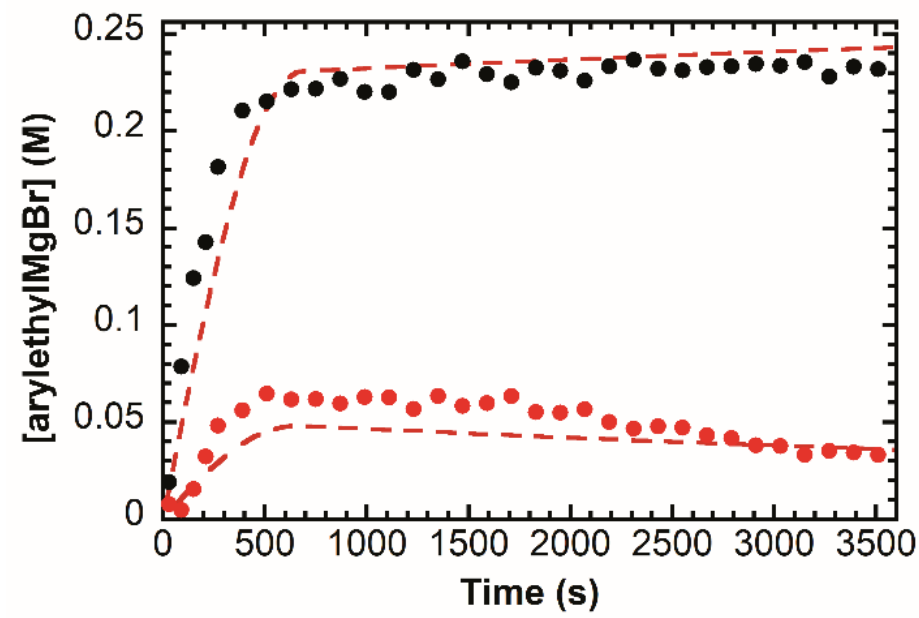

Figure 2.21: Predictions from COPASI for the isomerization of linear styrene with high concentrations of Grignard, [(1-phenylethyl) $\mathrm{MgBr}]$ vs time $(\bullet)$ and [(2phenylethyl)MgBr] vs time (-). Conditions: $0.291 \mathrm{M} \mathrm{S} ; 2.91 \mathrm{mM}\left(\mathrm{PDI} \mathrm{IPr}^{\mathrm{i}} \mathrm{FeCl}_{2} ; 0.434\right.$ M G; 1 hour; THF; $25^{\circ} \mathrm{C}$.

Rationalizations for the complex observed kinetic dependencies were determined by simulating timecourse data under various concentration regimes using the collection of elementary steps and rate constants (Table 2.10). When the concentration of catalyst was varied at a higher concentration of Grignard substrate (3 equiv), the non-linear catalyst dependence became linear, Figure 2.22A. Gratifyingly, these prediction results were 
reinforced experimentally (Figure 2.22A). Further, when the model was altered such that Step 7 (transmetallation to form $L$ ) was irreversible, the catalyst dependence also became linear (Figure 2.22B), strongly suggesting that the hyperbolic catalyst dependence, measured by initial rates, is a consequence of a complex dynamic between active catalyst and linear Grignard product as catalyst loading increases.

The kinetic model in Scheme 2.8 was then applied to Thomas' 2012 reported reaction timecourse using $\mathrm{G}^{\mathrm{Et}}$, under an $\mathrm{N}_{2}$ gas. ${ }^{153}$ The predicted timecourse fits the data well given that off-cycle low valent iron-dinitrogen species were observed and characterized by Thomas/Neidig (Scheme 2.5 and Figure 2.23). ${ }^{195}$ This demonstrates the likelihood that this kinetic model provides a mechanistic foundation by which similar transfer hydrometallation reactions with different Grignard reagents or alkene derivatives can be understood. These trends will be further discussed in Chapters 3 and 4 . Catalytically competent low-valent iron species resulting from decomposition of PDI-iron active catalyst were not considered in the kinetic modeling, specifically the generation of Thomas/Neidig's $\left(\eta^{2} \text {-styrene) }\right)_{3}$ iron(0)-benzylic anion. ${ }^{195}$ Deviation from agreement with experimental data may reflect the contribution of secondary catalytic pathways not accounted for in the proposed mechanism in Scheme 2.8.

A

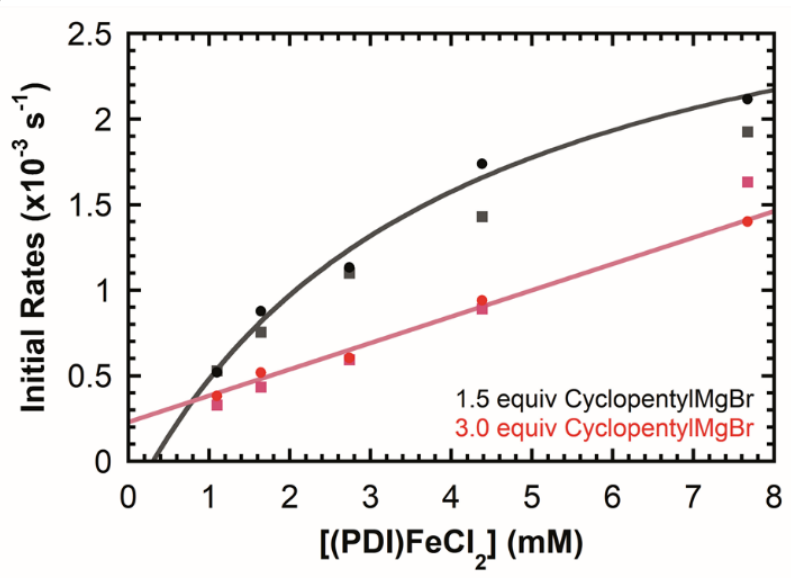

B

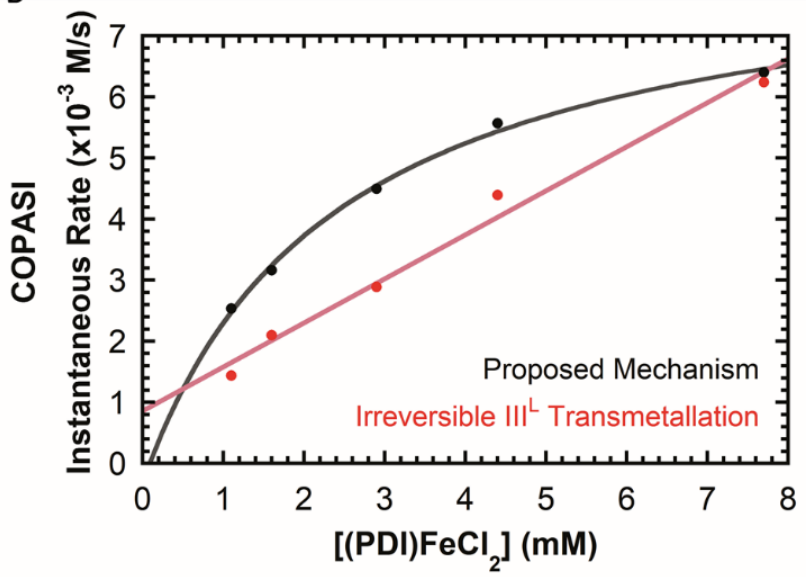

Figure 2.22: Kinetic predictions based on reactions and rates tabulated in Table 2.1. (A) Non-linear and linear initial rate catalyst dependencies (experimental data •; predicted data - ) under standard catalytic conditions. (B) Instantaneous Rates vs [(PDI)FeCl 2$]$ (Our Mechanism - ) and (Irreversible Linear Trans-metallation - -). Conditions: $0.291 \mathrm{M} \mathrm{S}$; x mM (PDIPr)FeCl2; $0.434 \mathrm{M} \mathrm{G} ; 1$ hour; THF; $25^{\circ} \mathrm{C}$. 


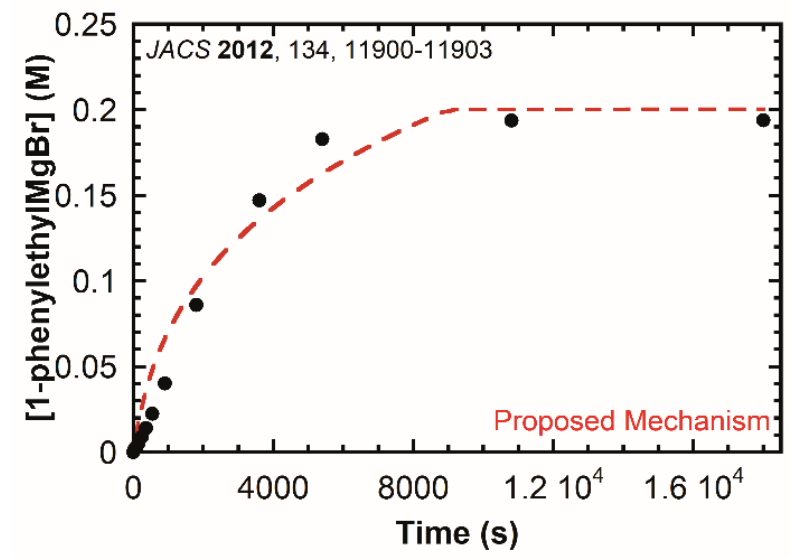

Figure 2.23: Timecourse data reported by Thomas with fit to proposed mechanism: Conditions: $0.23 \mathrm{M}$ styrene, $0.5 \mathrm{mM}\left(\mathrm{PDI}{ }^{\mathrm{PPr}}\right) \mathrm{FeCl}_{2}, 0.35 \mathrm{M}$ Grignard, 5 h, THF, rt, N2 (1 atm). ${ }^{153,195}$

Through similar timecourse simulations, specifically the removal of Steps 6-9 (formation of linear Grignard, L, and trapping iron catalyst off-cycle, IIIBR.S), the observed rate vs. concentration behavior no longer qualitatively reflected the experimentally observed inhibitory behavior of Grignard and styrene substrates at low concentration (Figure 2.24), as seen in the kobs dependencies (Figure 2.4D and 2.4F). Owing to the absence of mirrored kinetic behavior, the processes of isomerization and formation of key off-cycle intermediates were determined to play a crucial role in the complex observed substrate dependence kinetics.
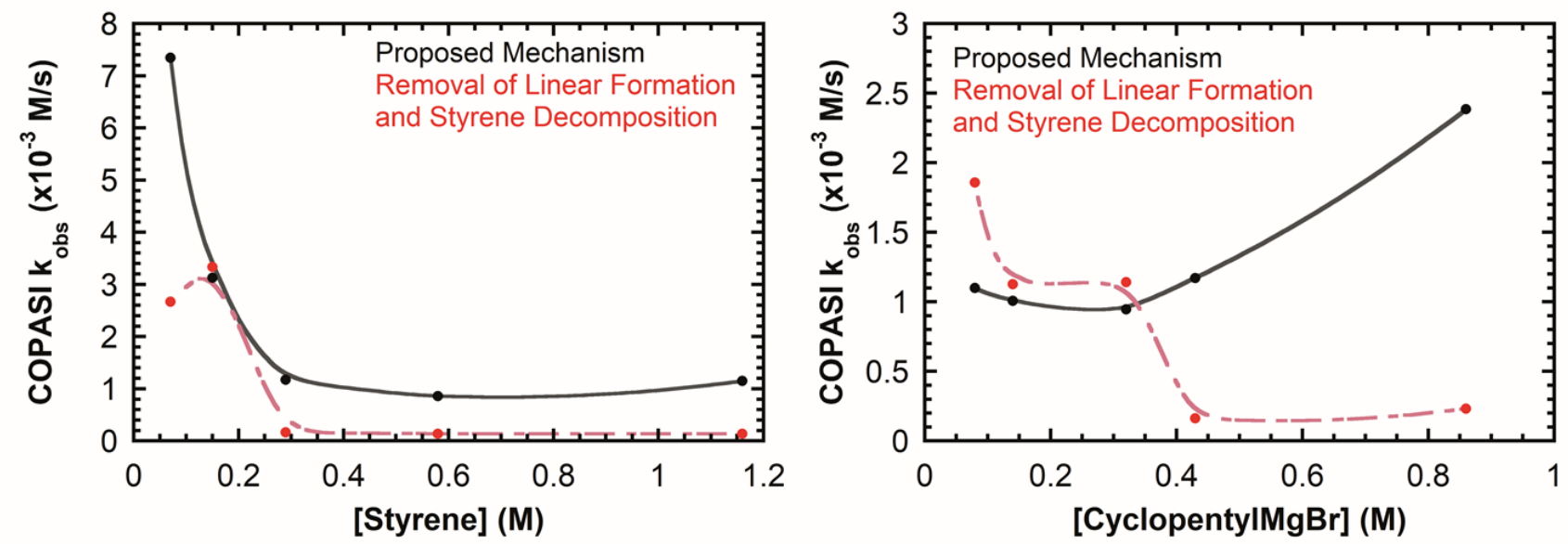

Figure 2.24: Kinetic predictions based on reactions and rates tabulated in Table 2.10. (A) Predicted styrene dependencies upon changing mechanism (proposed mechanism data •; changed mechanism data •) under standard catalytic conditions. (B) Predicted cyclopentyl $\mathrm{MgBr}$ dependencies upon changing mechanism (proposed mechanism data $\bullet$; changed mechanism data $\bullet$ ) under standard catalytic conditions. 
Finally, Scheme 2.8 depicts a concise mechanism consisting of the 12 elementary steps of hydromagnesiation. Based upon recent work by Chirik and Thomas/Neidig, formal (PDI)iron(0) species results rapidly upon treatment of iron(II) pre-catalyst with $>20$ equiv. of Grignard reagent (GCYP). ${ }^{95,195,202-204}$ Thus, a formal iron(0) species I, ligated by cyclopentene (CYP) or solvent (THF), enters the catalytic cycle through competing pathways of ligand substitution. Pathway 1 occurs with an equivalent of Grignard reagent, under limiting styrene conditions, to form first an adduct $1 \cdot G$ which enters the catalytic cycle as iron-alkyl II. The second pathway, ligand substitution with styrene (S), yields $1 \cdot S$ that subsequently undergoes transmetallation and enters the catalytic cycle as styrene-bound iron-alkyl $\| \cdot S$. Intermediate $\| \cdot S$ undergoes competing 2,1- and 1,2-hydride transfer pathways to yield iron-benzyl, IIIBR, and iron-homobenzyl, IIIL. The rate constants for the forward reactions indicate approximately 6.1 -fold faster formation of III ${ }^{\mathrm{BR}}$, Table 2.10. The irreversibility of steps 4,6 , and 9 is consistent with experimental observations made with cyclopentene and other disubstituted alkenes by Thomas. ${ }^{153,170,195}$ Transmetallation of $\mathrm{III}^{\mathrm{BR}}$ with $\mathrm{G}^{\mathrm{CYP}}$ is favored over IIIL. The reversibility of transmetallation of IIIL (step 7) leads to the irreversible isomerization of $\mathrm{II}^{\mathrm{L}}$ to $\mathrm{II}^{\mathrm{BR}}$ in the presence of excess styrene. The irreversibility of Step 5, transmetallation of $\mathrm{III}^{\mathrm{BR}}$, is consistent with previous deuterium studies discussed by Thomas, wherein branched Grignard product does not reenter the catalytic cycle (Scheme 2.3B). 153,170,195 Finally, IIIBR may react with another equivalent of styrene to form off-cycle alkene adduct III ${ }^{\mathrm{BR}} \cdot \mathrm{S}$. Such a species would be necessary to access the catalytically competent off-cycle $\left(\eta^{2}\right.$-styrene ${ }_{3} \mathrm{Fe}^{0}($ benzyl) anion intermediate identified and independently prepared by Thomas and Neidig. ${ }^{195}$ Their study showed this species accounted for a small percentage of catalytically active iron at early time points then increased steadily over the course of the reaction, up to $38 \%$ of iron speciation for $\mathrm{m}$ methoxystyrene. ${ }^{195}$ This observed catalyst decomposition behavior is consistent with approximately $10^{3}$ slower formation of $\mathrm{IIIR}^{\mathrm{BR}} \mathrm{S}$ relative to formation of branched Grignard product via transmetallation (Step 5, Table 2.10). 


\subsection{Conclusions}

In summary, one of the first in depth mechanistic studies of hydromagnesiation, an underutilized class of alkene hydrofunctionalization reactions, has been described by this study. Using in situ infrared spectroscopic studies and global numeric modeling, a detailed understanding of the kinetic complexities of hydromagnesiation of styrene by an iron-PDIPr catalyst has been achieved. The following chapters will aim at identifying the electronic/steric features of the sacrificial organometallic reductant and alkene substrate that lead to efficient and selective catalysis.

\subsection{Comments}

The discussion of this work has now been accepted by Organometallics. ${ }^{1}$ 


\section{Chapter 3. Iron-catalyzed hydromagnesiation: Effects of styrene}

3.1. Introduction

In 2012, Thomas and Greenhalgh demonstrated that a variety of vinyl arene substrates efficiently underwent hydromagnesiation. These substrates, most of which displayed electron donating (EDG) character, with ethylMgBr (GEt) yielded high regioselectivity for the branched Grignard/carboxylic acid regioisomer (BR). ${ }^{153,170,195}$ Scheme 3.1 illustrates a small selection of the overall reaction substrate scope published in 2012. ${ }^{153}$ Electron deficient substrates, such as p-fluoro styrene, resulted in low yields and high branched regioselectivity. In 2016, Greenhalgh began to develop a discussion of reactivity based upon the electronic nature of the vinyl arene. ${ }^{170}$ Using a Hammett correlation, Greenhalgh determined that electron rich substrates were favored which was substantiated by a largely negative $\rho$ value $(\rho=-2.2) .{ }^{170}$ Such a large $\rho$ value generally describes a buildup of positive charge in the transition state, possibly at the benzylic position of styrene. This characterized analysis suggests that hydride transfer would not be the turn-over limiting step (TLS). As discussed in Chapter 2 and by Thomas and coworkers, ${ }^{153,170,195}$ the TLS was concluded to be transmetallation to generate the BR. However, this does not discuss the reaction selectivity for EDG-substituted styrenes. In 2016, Greenhalgh described two intermediates that would explain the electronic selectivity. ${ }^{170}$ First, EDG-substituted styrenes favor the catalytically-active mono-styrene iron intermediate whereas electron deficient styrenes would favor a bis-styrene iron species that is catalytically inactive and may lead to decomposition (Scheme 3.2). ${ }^{170}$ Second, owing to increased back-bonding of a reduced iron center to an electron deficient styrene, the hydride transfer from the alkyl group to the $\beta$-position of the vinyl group would be difficult due to the increased probable metallocyclopropane character. ${ }^{170}$ These suggestions and further descriptions of the reactivity with styrene derivatives is the aim of this chapter to develop a better understanding of the electronic selectivity of ironcatalyzed hydromagnesiation. 


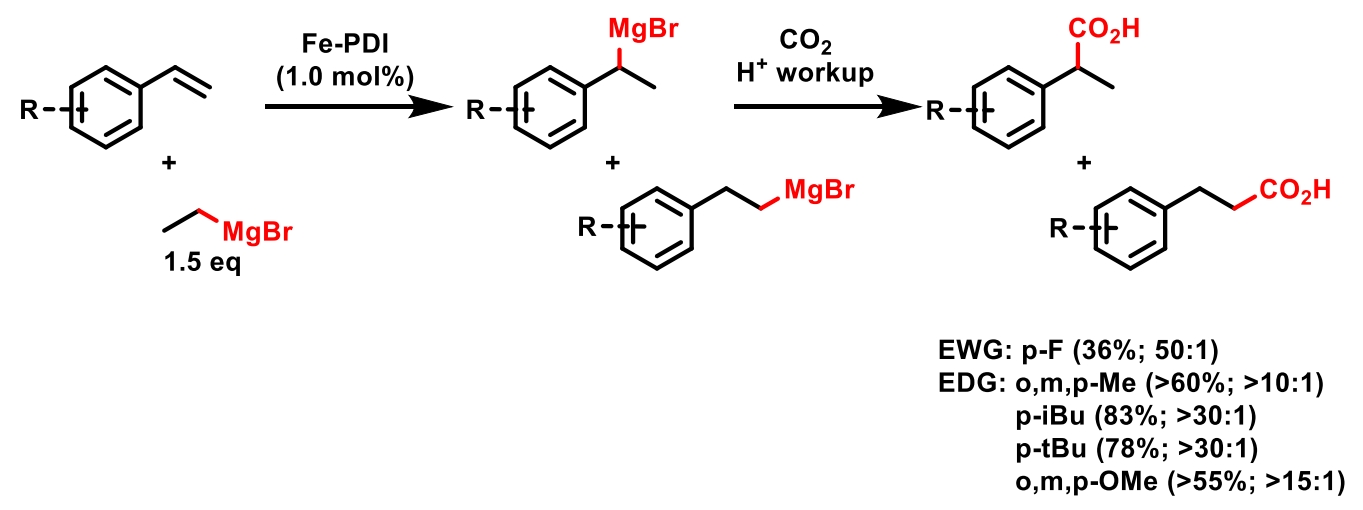

Scheme 3.1: Thomas' Hydromagnesiation. Conditions: anhydrous $\mathrm{FeCl}_{2}(1 \mathrm{~mol} \%)$, PDI ${ }^{\text {Pr }}$ ligand (1 mol\%), S (1.0 eq), GEt (1.5 eq), THF, N2 (1 atm), rt, 2 h. Quench: 1) $\mathrm{CO}_{2}$ (dry), rt, 1h; 2) $1.0 \mathrm{M} \mathrm{HCl}$, rt, overnight. ${ }^{153}$

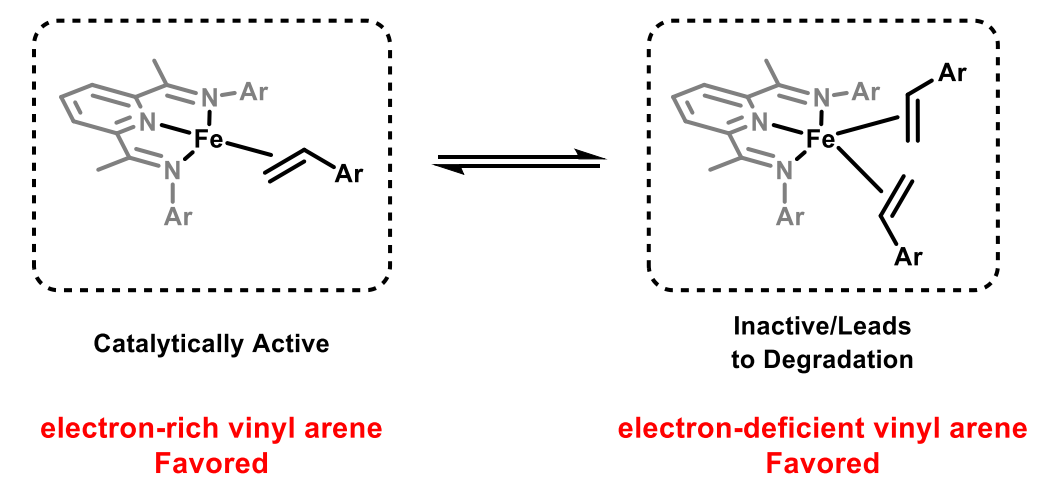

Scheme 3.2: Thomas' possible explanations for a negative $\rho$ value for iron-catalyzed hydromagnesiation of electronically different vinyl arenes. ${ }^{153,170,195}$

\subsection{Results}

Using the initial rates and observed rates analyses developed in Chapter 2, the concentration effects of a variety of para-substituted electron rich styrenes were studied. Our analysis began with the comparison of $p$-substituted Grignard reagents and the hydromagnesiated time courses. Unfortunately, upon analysis of the independently synthesized (\{p-substituted\}aryl)ethyl Grignard, the absence of a signal within the region of $1610-1570 \mathrm{~cm}^{-1}$ suggested a new stretching frequency was needed for analysis. The region of $1500 \mathrm{~cm}^{-1}$ for each $p$-substituted styrene was used for the remainder of the analysis in this chapter, as seen in Figure 3.1. The initial rates were normalized for comparison with styrene. Following the same IGOR deconvolution methods, the individual timecourses for each styrene derivative were calculated and used for initial rate and observed rate kinetic analyses. 


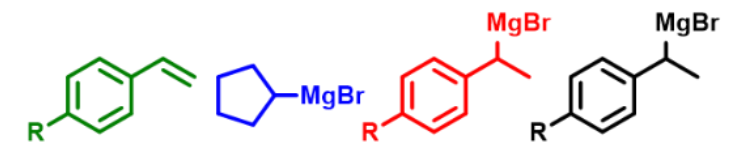

A.

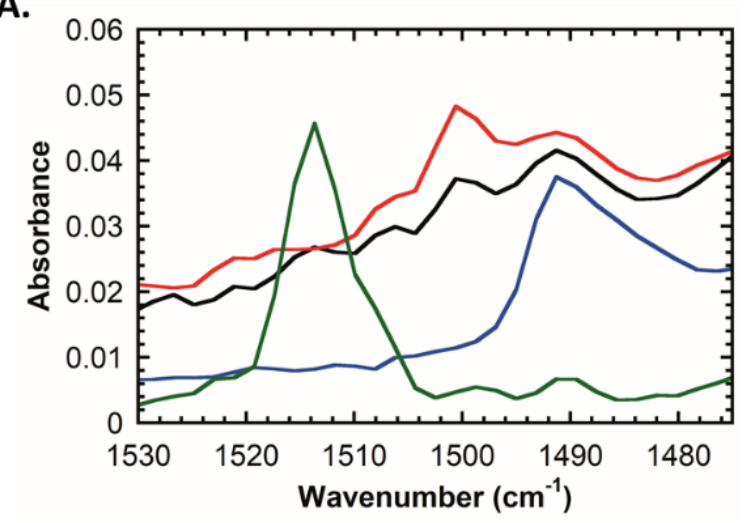

B.

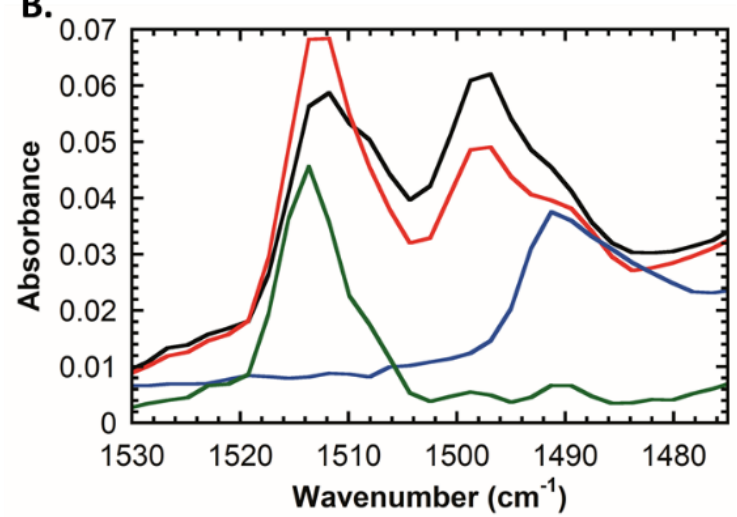

Figure 3.1: Frequency analysis of the absorbances of the starting material and the product from Scheme 3.1. Absorbance vs wavenumber. A) p-methylstyrene; B) pmethoxystyrene

A.
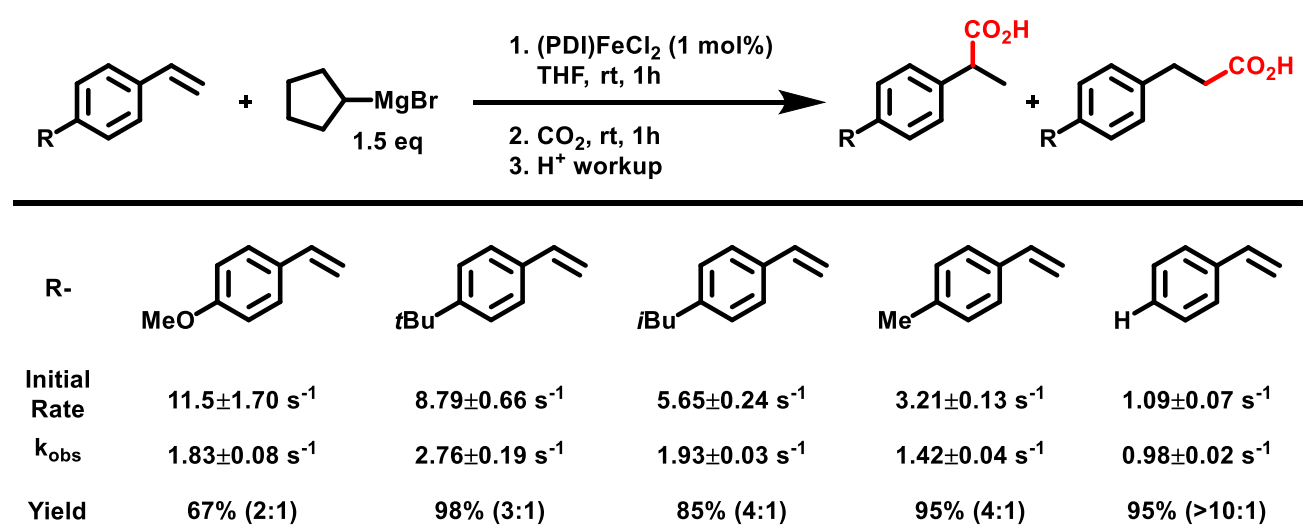

B.

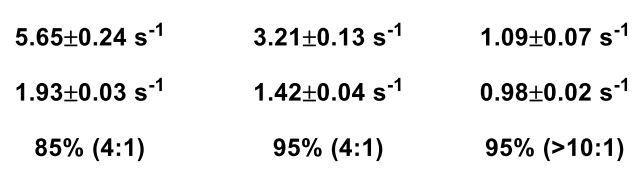

C.
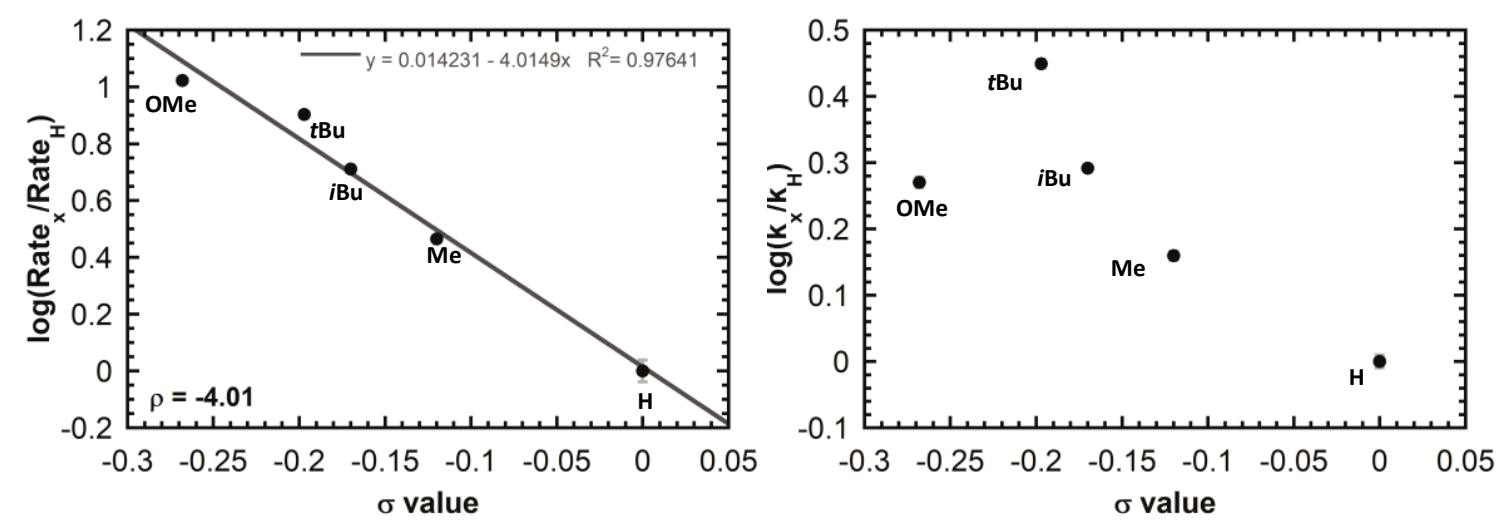

Figure 3.2: Hammett Parameters calculated from the initial rate and observed rates analyses: A) Reaction conditions and yields; B) Initial rates Hammett Correlation; C) observed rate correlation. 
Figure 3.2B corroborates Thomas's findings of a large $\rho$ value for the initial rate Hammett correlation. Surprisingly, when using a Hammett correlation to compare the observed rate kinetics, a complex arrow shaped correlation is noted, Figure $3.2 \mathrm{C}$. This would suggest that the mechanism of the reaction would change as the electron donating character increases. ${ }^{205,206}$ Without any justification for this kinetic complexity, concentration dependencies of multiple electron rich vinyl arenes were probed. Thomas and Neidig, following the groundwork laid down by Greenhalgh, began characterizing the concentration dependencies of 3 vinyl arenes: p-tert-butylstyrene, m-methoxystyrene, and o-methoxystyrene. ${ }^{170,195}$ Their findings at low concentrations of catalyst describe a saturation dependence on each substrate, styrene (S) and ethylMgBr $\left(G^{E t}\right) .{ }^{170,195}$ This may allude to the reaction complexity defined in Chapter 2. Concentration dependencies were identified for four electron rich substrates: $p$-methylstyrene $\left(\mathrm{S}^{\mathrm{Me}}\right), p$-isobutylstyrene $\left(S^{\mathrm{iBu}}\right)$, p-tert-butylstyrene $\left(\mathrm{S}^{\mathrm{tBu}}\right)$ and $p$-methoxystyrene $\left(\mathrm{S}^{\mathrm{OMe}}\right)$. These vinyl arene derivatives resulted in saturation initial rate dependencies. However, in comparison to the initial rate dependence of styrene $(S)$, the trends also do not bisect the origin (Figure 3.3A). As with the discussion of $S$ dependence and its complexities in Chapter 2, the resultant non-zero intercept saturation dependence of the vinyl arenes may still allude to a competing activation pathway. The observed rate dependencies of each derivative also mimic the inhibition at low concentrations of S (Figure 3.3B). This inhibition could still be attributed to the off-cycle reaction of vinyl arene with iron-benzyl. Further COPASI timecourse modeling and parameter estimation would aim to deconvolute the electronic specificity of iron-catalyzed hydromagnesiation utilizing the mechanism determined in Chapter 2 (Reproduced as Scheme 3.3 for convenience). 


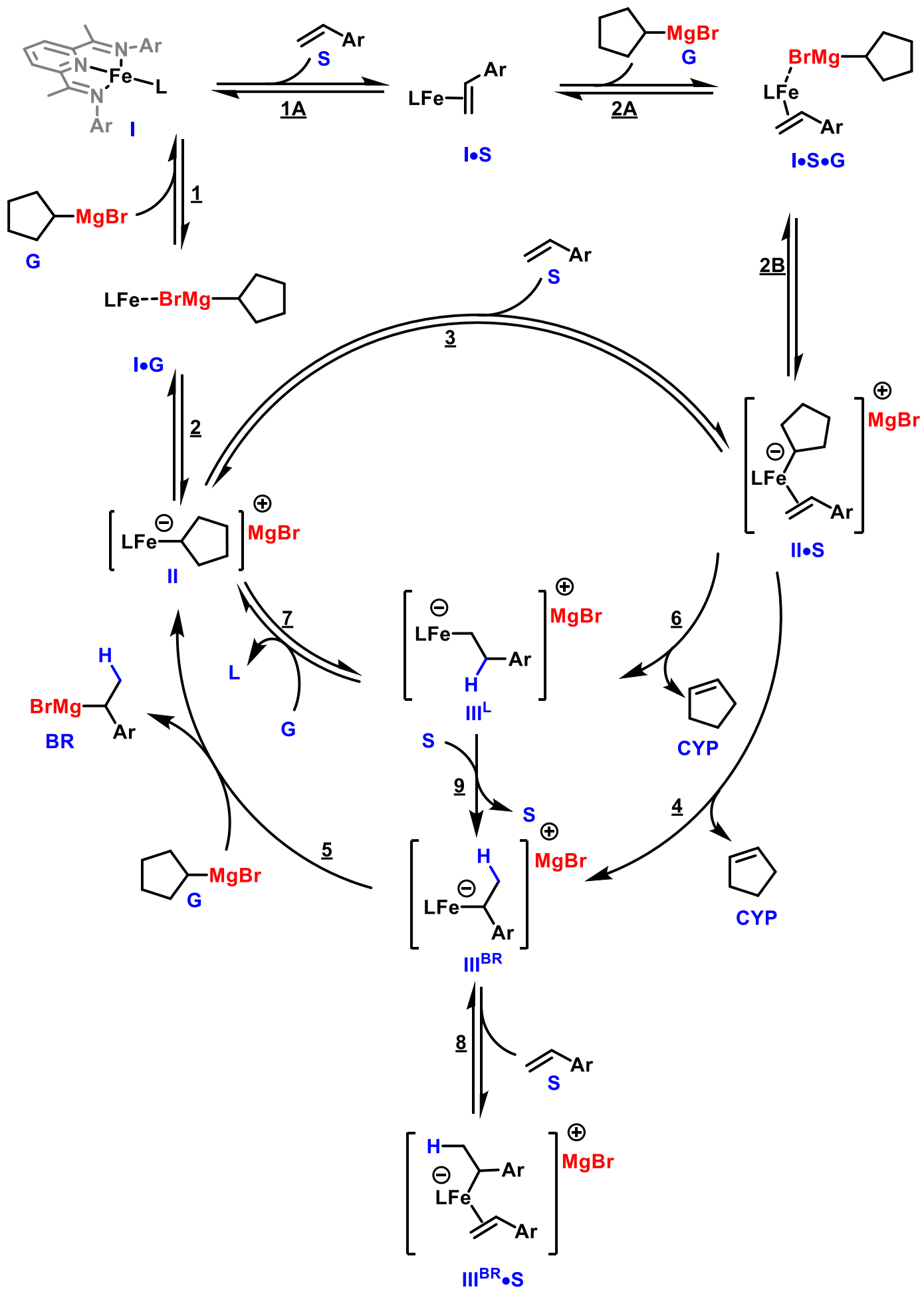

Scheme 3.3: Proposed mechanism of iron-catalyzed hydromagnesiation. ( $L=P D{ }^{i P r}$, $\mathrm{L}=$ linear isomer) 
Table 3.1: Calculated rate constant ratio $\left(\mathrm{k}_{f} / \mathrm{k}_{\mathrm{r}}\right.$ or $\mathrm{k}_{\mathrm{f}}$ for irreversible reactions) from COPASI for Scheme 3.3

\begin{tabular}{|c|c|c|c|c|c|}
\hline $1 \mathrm{~A}$ & $8.64 \times 10^{4}$ & $1.11 \times 10^{5}$ & $4.23 \times 10^{4}$ & $1.96 \times 10^{4}$ & $2.27 \times 10^{4}$ \\
\hline $2 \mathrm{~A}$ & 689.1 & $8.50 \times 10^{4}$ & $9.46 \times 10^{3}$ & $1.67 \times 10^{3}$ & $3.16 \times 10^{3}$ \\
\hline $2 B$ & $1.87 \times 10^{8}$ & $3.10 \times 10^{10}$ & $1.20 \times 10^{9}$ & $1.34 \times 10^{7}$ & $4.26 \times 10^{8}$ \\
\hline 1 & 0.0117 & 0.081 & 0.060 & 0.020 & 0.0013 \\
\hline 2 & $2.74 \times 10^{3}$ & $5.47 \times 10^{5}$ & $5.49 \times 10^{3}$ & $8.83 \times 10^{3}$ & 129.6 \\
\hline 3 & 2.51 & 1.30 & 1.36 & 1.04 & 4.72 \\
\hline 4 & 1.62 & 2.849 & 1.89 & 1.22 & 1.35 \\
\hline 5 & 8.25 & 13.31 & 6.64 & 2.83 & 1.41 \\
\hline 6 & 1.66 & 2.29 & 0.654 & 0.394 & 0.22 \\
\hline 7 & 0.0868 & 16.9 & 4.13 & 0.673 & 0.0089 \\
\hline 8 & 18.4 & $2.13 \times 10^{3}$ & 957.0 & 30.8 & 35.5 \\
\hline 9 & 0.042 & 0.378 & 0.014 & 0.019 & 0.0084 \\
\hline
\end{tabular}

A.

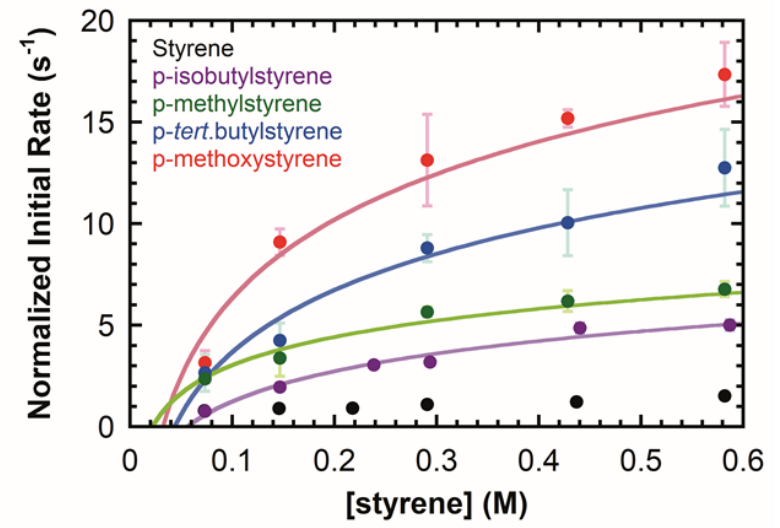

B.

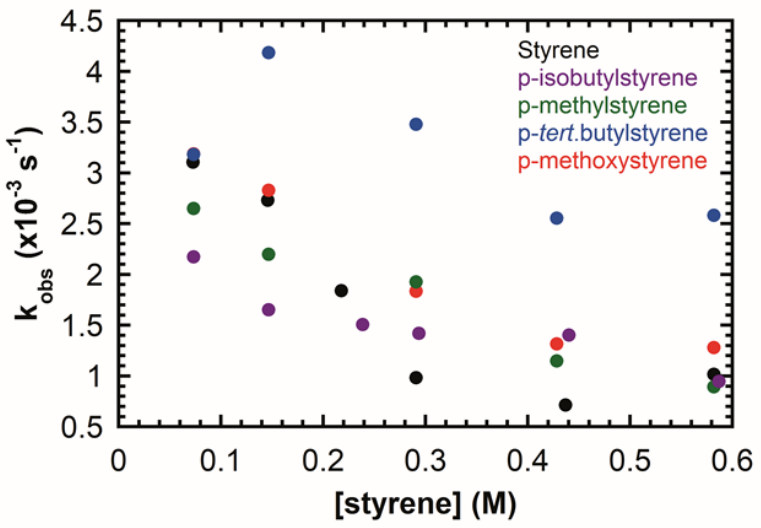

Figure 3.3: Concentration dependencies of various styrene derivatives: A) Initial rates; B) observed rate.

Upon estimating individual COPASI parameters for each styrene derivative using the mechanism in Scheme 3.3, calculated timecourses for six concentration variants for each derivative resulted in substantial to excellent fit (Figure 3.4 and $k=0.67-0.9)$. COPASI calculated rate parameters for each derivative can be used to assess the overall calculated rate constant ratio $\left(\mathrm{k}_{\mathrm{f}} / \mathrm{k}_{\mathrm{r}}\right)$. This ratio can compare key intermediate steps to describe the complex experimental kinetic analyses. For the reactions that are described 
as irreversible (4-6 \& 9), the forward reaction calculated rate constant was used in the comparison. Based upon the initial rates analyses of each styrene derivative (Figure 3.3A) it could be suggested that dual initiation pathways are still viable and that the path containing styrene activation was still significantly faster than initiation by Grignard. Consequently, once the effect of catalytic turnover was addressed (steps 3-9) there were noticeable differences among the rate constants and TLS based upon the observed rate constant Hammett correlation (Figure 3.2B). The comparison of rate constant ratios is shown in Table 3.1.
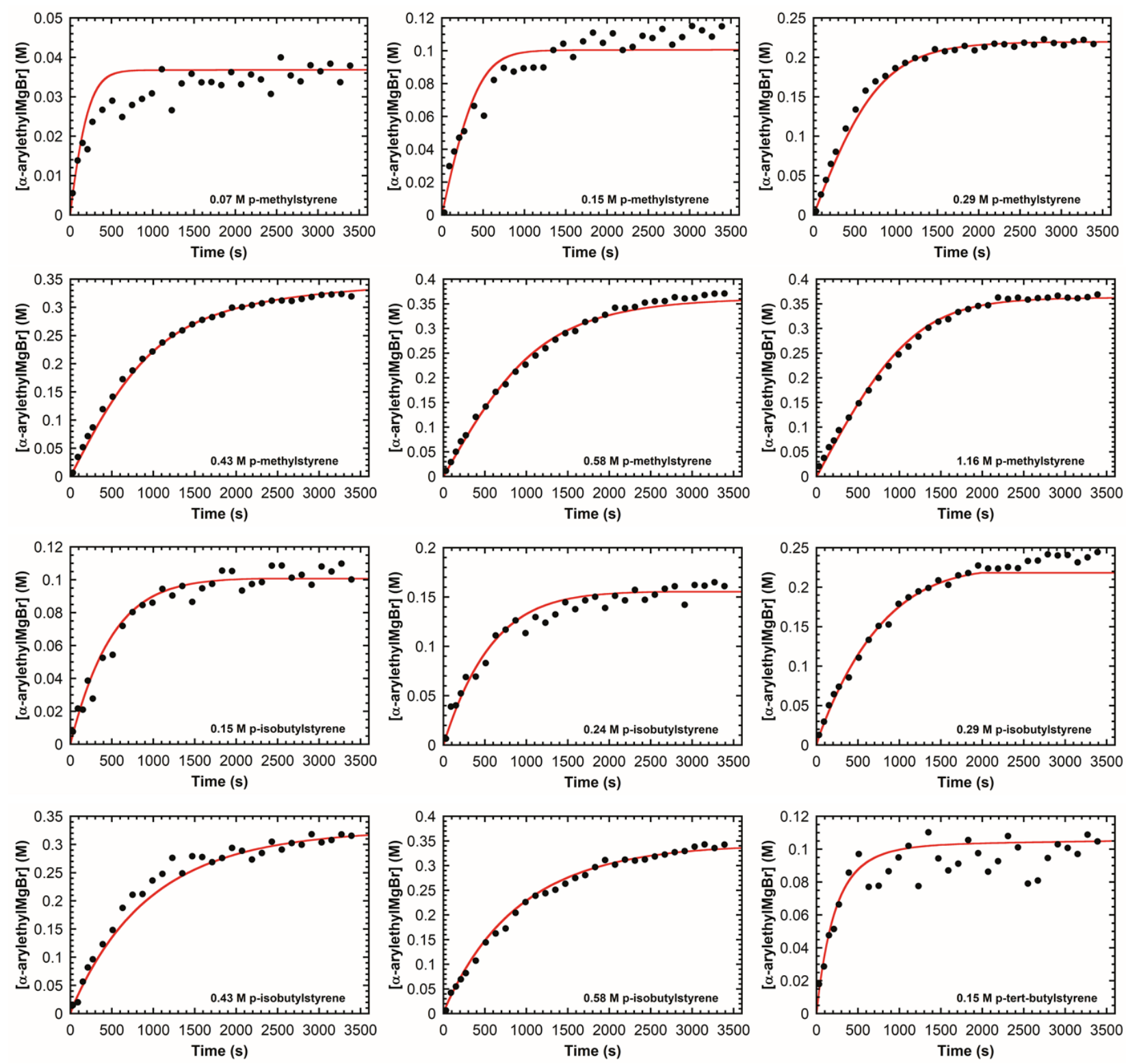

${ }^{*}$ Figure 3.4 continued on page 66

$-65-$ 

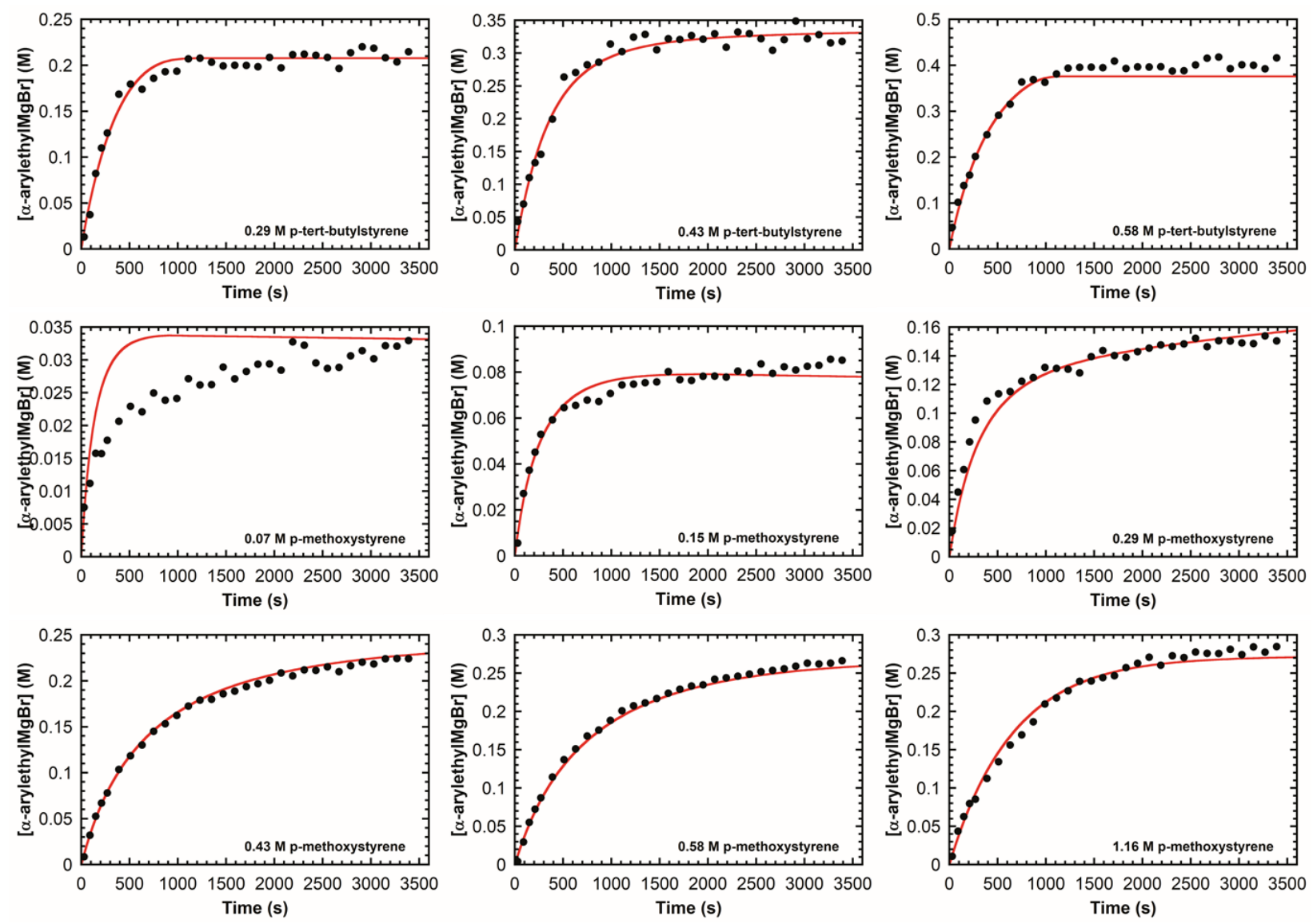

Figure 3.4: COPASI calculated Reaction time courses, [(1-(4-substituted) phenylethyl)MgBr] vs time. Conditions: x M 1; 2.9 mM (PDIPr) $\mathrm{FeCl}_{2} ; 0.434 \mathrm{M} \mathrm{2;} 1$ hour; THF; rt. *See Figure for derivative concentrations. Cohen's kappa: $\mathrm{S}^{\mathrm{Me}}$ : substantial agreement $\kappa_{M e}=\frac{(83 \%)-(50 \%)}{1-50 \%}=0.67$; SiBu: excellent agreement $\kappa_{i B u}=\frac{(95 \%)-(50 \%)}{1-50 \%}=$ $0.90 ; \mathrm{S}^{\text {tBu}}$ : excellent agreement $\kappa_{t B u}=\frac{(90 \%)-(50 \%)}{1-50 \%}=0.80 ;$ S ${ }^{\text {OMe: }}$ substantial agreement $\kappa_{O M e}=\frac{(86 \%)-(50 \%)}{1-50 \%}=0.72$

As the EDG character increased, the rate constants of steps 4-9 increased. This interesting result is concluded to be a new competition between the styrene insertion pathways. This competition yielded in lower regioisomer ratios seen experimentally. Though this does provide an explanation for the regioselectivity of each analyzed styrene derivative, it does not fully explain the reduced yields resulting from SOMe. In 2012, Thomas and Greenhalgh commented on a reduced yield with 3,4-dimethoxystyrene. They stated that this reduction in yield is "possibly due to Grignard-mediated demethylation." 153 
This Grignard-mediated side reaction may also attribute to the decreased yield for SOMe. The close association of an electrophilic metal center to the $p$-alkoxy group, generating a possible oxonium in solution, may introduce a higher $\sigma$-value $(\sigma>-0.2)$ when compared to a neutral alkoxy group. A similar discussion of a change in $\sigma$-value based upon the introduction of a positive charge has been noted upon changing a $p$-dimethylamine $(\sigma=$ $-0.86)$ to a $p$-trimethylammonium $(\sigma=0.82) .{ }^{207}$

With the assistance of Prof. Brian Popp, the natural charges were calculated using Gaussian 16 and NBO 3.1 for the benzylic carbon in the vinyl arene as well as the benzylic anion. ${ }^{208,209}$ Upon association of an electrophilic metal center to the $p$-alkoxy group, the electron rich character of the vinyl arene decreases, changing the overall relationship of the linear free energy, thus, resulting in a $\sigma$-parameter more closely related to an EWG. Figure 3.5 shows the relationship of the calculated natural charges and the Hammett correlation value $(\sigma)$.
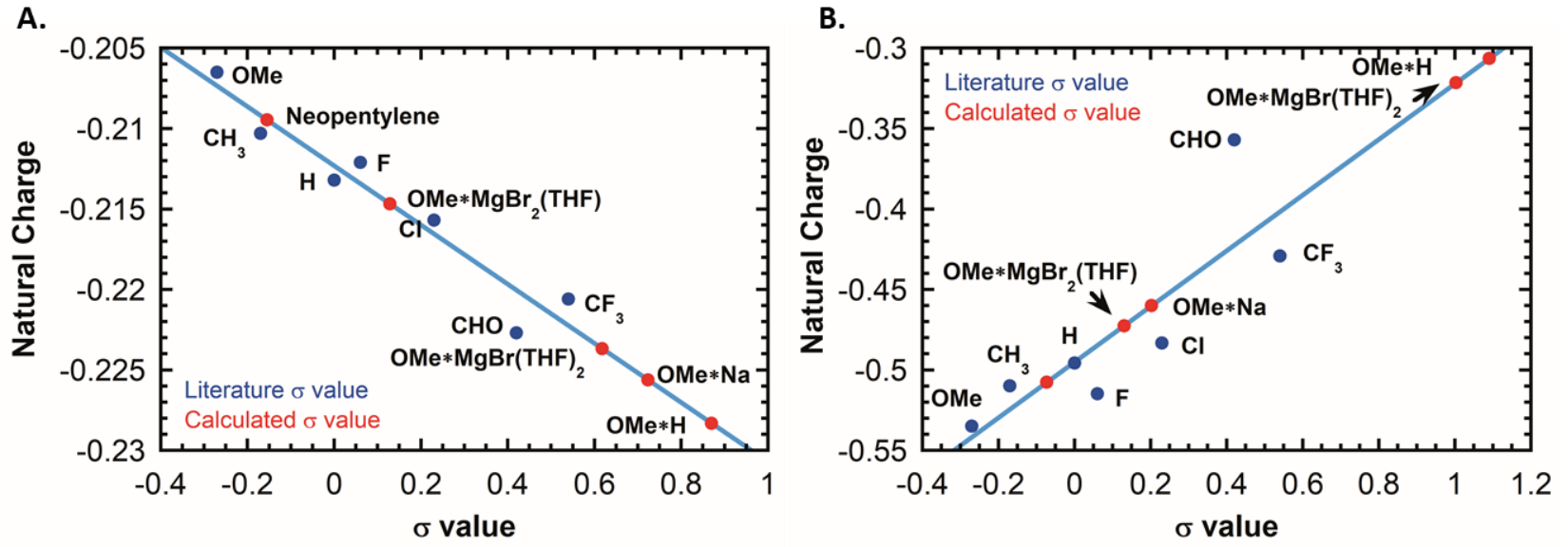

Figure 3.5: Calculated natural charge of the benzylic carbon relative to the $\sigma$-value. A) bencylic-C of vinyl arene; B) benzylic-C anion. (DFT method using Gaussian 16 M06-2X/6-311+G(2d,p)//M06-2X/6-31G(d). SMD solvent model used for tetrahydrofuran solvation corrections. Natural charges calculated using NBO 3.1. Calculations performed by Professor Brian Popp.) $)^{208,209}$ 


\subsection{Conclusions}

In summary, we have extended the iron-catalyzed hydromagnesiation of styrene to include a variety of electron rich vinyl arenes. The mechanism of hydromagnesiation fits remarkably well to multiple timecourses. However, the reduced reactivity of $p$ methoxystyrene could be attributed to either 1) the formation of a formal "oxonium" by association with an electrophilic metal center, $\mathrm{Mg}$, or 2) the TLS changes from the transmetallation of the benzylic-iron species to the competing insertion pathways as evidenced by the rate constants of step 4 and 6 being 7 -fold slower than step 5 . 


\section{Chapter 4. Iron catalyzed-hydromagnesiation: Effects of Grignards}

\subsection{Introduction}

Throughout the past seven years, Thomas and coworkers have identified Grignard reagents that can act as sacrificial hydride and reductant sources. ${ }^{153,170,195}$ Their initial rate studies resulted in the identification of ethylMgBr $\left(G^{\mathrm{Et}}\right)$ as the most efficient Grignard source (Scheme 4.1). ${ }^{153,170,195}$ In 2019, Thomas/Neidig characterized two intermediates within the catalytic cycle with $\mathrm{GEt}^{\mathrm{E}}$ and styrene, S. ${ }^{195}$ The molecular structures characterized by x-ray crystallography (Figure 4.1) and the Mössbauer spectral data of these intermediates aided in the identification of the formal charge on iron. Characterized as a formal iron(0)-ate complex, the (PDIPr)iron-(ethyl) ethylene species in Figure 4.1 A was determined to be a viable catalyst for the hydromagnesiation process. ${ }^{195}$ Upon dissociation of ethylene, styrene coordinates to the (ethyl)iron(0)-ate species and subsequently transfers a $\beta$-hydride. According to Thomas/Neidig the reversibility of hydride transfer is dependent upon the ability of the generated alkene to undergo hydrometallation. The viability of the homobenzylic Grignard, L, as a hydride source was concluded to be one order of magnitude slower than $\mathrm{G}^{\mathrm{Et}}$. This was substantiated by our studies in Chapter 2. ${ }^{153,170,195}$ Stoichiometric reaction of $\left(\mathrm{PDI}{ }^{\mathrm{PPr}}\right) \mathrm{FeCl}_{2}$ with excess $\mathrm{L}$ resulted in the generation of a homobenzylic(dinitrogen) iron(0)-ate species, suggesting that the isolation of a discrete (PDIPr)iron-(alkyl) styrene species is unfavorable, Figure 4.1B. ${ }^{195}$ The absence of the formation of an iron-alkyl styrene complex suggests that steric interactions impede the coordination of styrene and hydride transfer does not occur through the complex in Figure 4.1B. ${ }^{195}$ Other Grignard studies such as the use of isobutyl, isopropyl-, and cyclopentylMgBr (GiBu, $\mathrm{G}^{\mathrm{iPr}}$ and GCyp, respectively) were also studied by Thomas/Greenhalgh/Neidig. The effect of Grignard on the reaction was stated to be "highly dependent upon the structure" of the Grignard. ${ }^{195}$ These statements and a full analysis of a variety of primary and secondary Grignard reagents including $\mathrm{G}^{\mathrm{iBr}}$, $\mathrm{G}^{\mathrm{iPr}}$ and GCyp will be investigated within this chapter with the aim of determining trends in rate and efficiency of each Grignard. 


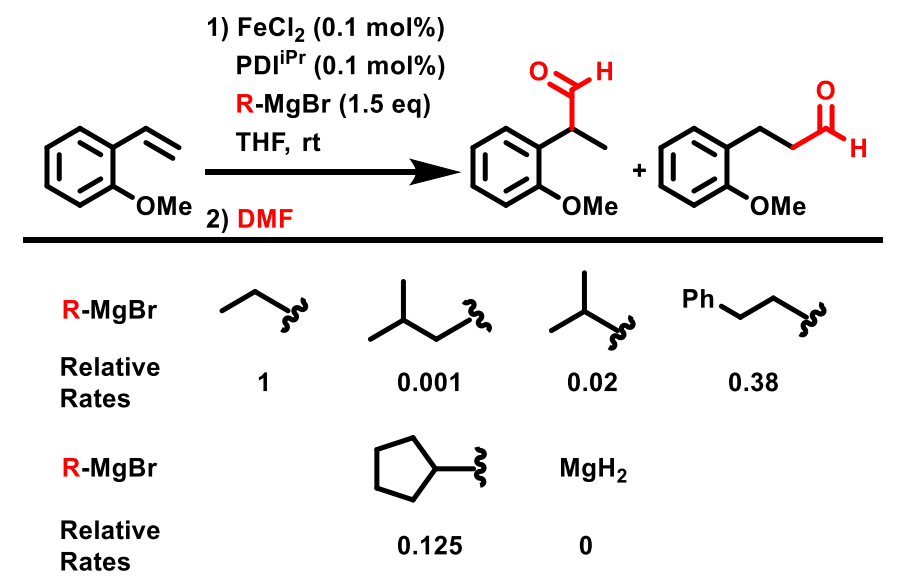

Scheme 4.1: Thomas' Hydromagnesiation. Conditions: anhydrous $\mathrm{FeCl}_{2}(0.1 \mathrm{~mol} \%)$, PDI IPr ligand (0.1 mol\%), S (1.0 eq), GEt (1.5 eq), THF, N2 (1 atm), rt, 2 h. Quench: 1) DMF (dry), rt, 1h; 2) $1.0 \mathrm{M} \mathrm{HCl}$, rt, overnight ${ }^{195}$

A.

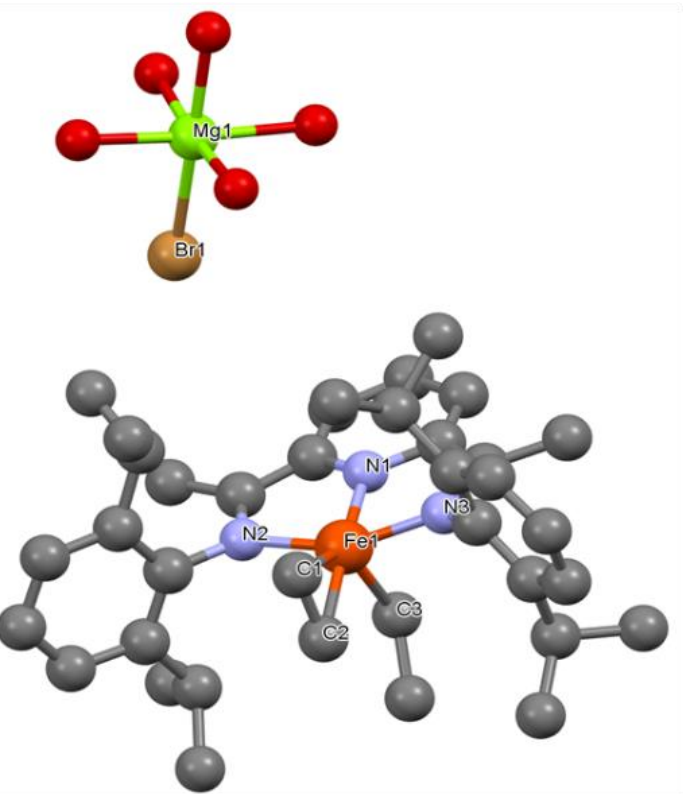

B.

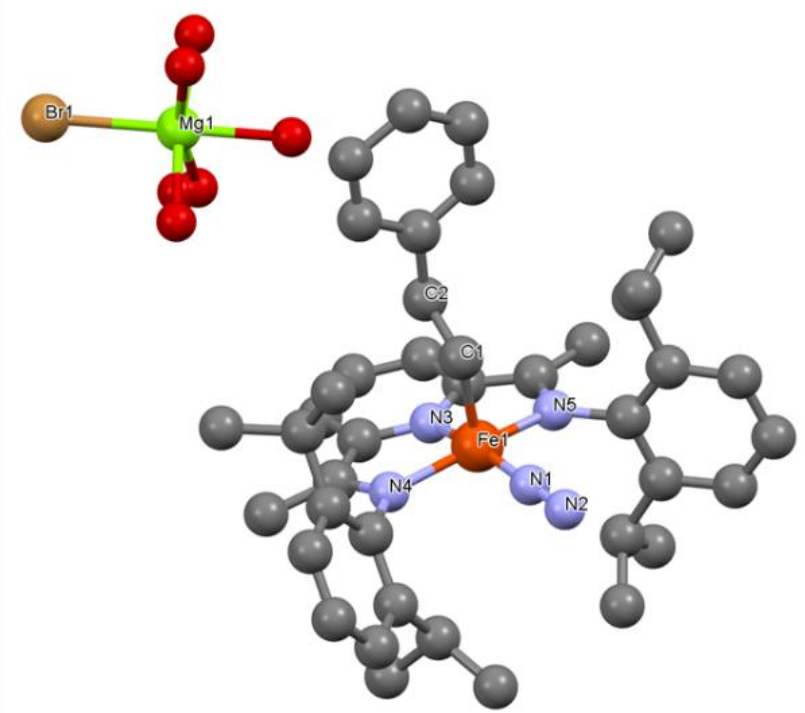

Figure 4.1: Molecular Structure of Thomas' Hydromagnesiation characterized intermediates: A) ethyl ethylene iron-PDI complex; B) homobenzylic iron-PDI dinitrogen complex. *Reproduced from CSD Entry: NOHHEA and NOHGUP. ${ }^{195}$ 
4.2. Results

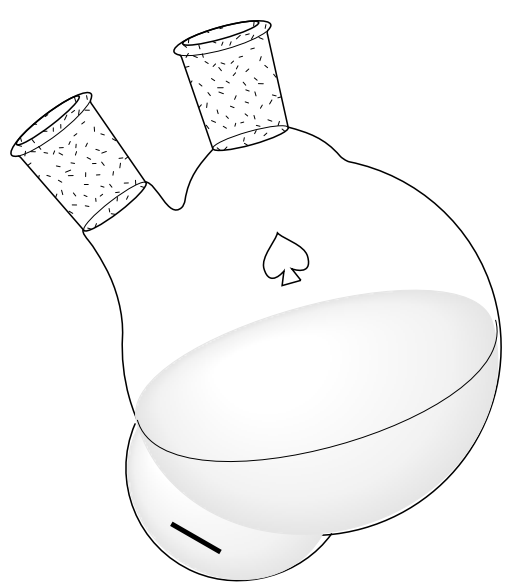

Figure 4.2: Flask used for hydromagnesiation when a gas is generated

Following the initial rates and observed rates analysis described in Chapter 2, the rates of hydromagnesiation with respect to Grignard were determined. However, certain Grignard reagents, such as ethyl Grignard, generate a gaseous alkene upon hydride transfer. These gaseous alkenes result in one main limitation: dissociation and evolution of the gas. To combat this known drawback, a stir rate dependence would introduce identification of a speed where gaseous alkenes are evolved proficiently. Specialty glassware was made to include a small bubble within the wall of the 2-neck flask used for catalysis (Figure 4.2). The small bubble aided in the determination of a stir rate dependence by allowing for increased stirring RPM without damaging the ReactIR Diamond composite (DiCOMP) probe, thus giving the best conditions for gas evolution. Using ethyl Grignard ( $G^{\mathrm{Et}}$ ), a Grignard known to produce high yields of the branched hydromagnesiated product, $\mathrm{BR}$, a stir rate dependence was used to determine the best stir speed for ethylene evolution (Figure 4.3A). Changing the stir rate resulted in no change in initial rate, but a linear increase in observed yield as well as an increase in overall yield. Although 1020 RPM yielded the best overall observed rate for the hydromagnesiation of styrene, the inconsistent behavior of the magnetic stir bar at such speeds was determined as troublesome and an inherent danger to the ReactIR DiCOMP probe. Therefore 820 RPM was used for all Grignard reagents that would evolve a gas. A comparison of the concentration dependencies of $\mathrm{G}^{\mathrm{Et}}$ at the standard stir rate (420 RPM) and the increased stir rate (820 RPM) resulted in interesting phenomena. Initial 
rates based upon the catalyst concentration became linear at higher stir rates, shown in Figure 4.3B, while the styrene concentration dependencies changed from inhibitory to first order non-zero intercept. These changes may result from inefficient dissociation of ethylene from the resting state of hydromagnesiation, suggested by Thomas/Neidig (Figure 4.1A). ${ }^{195}$

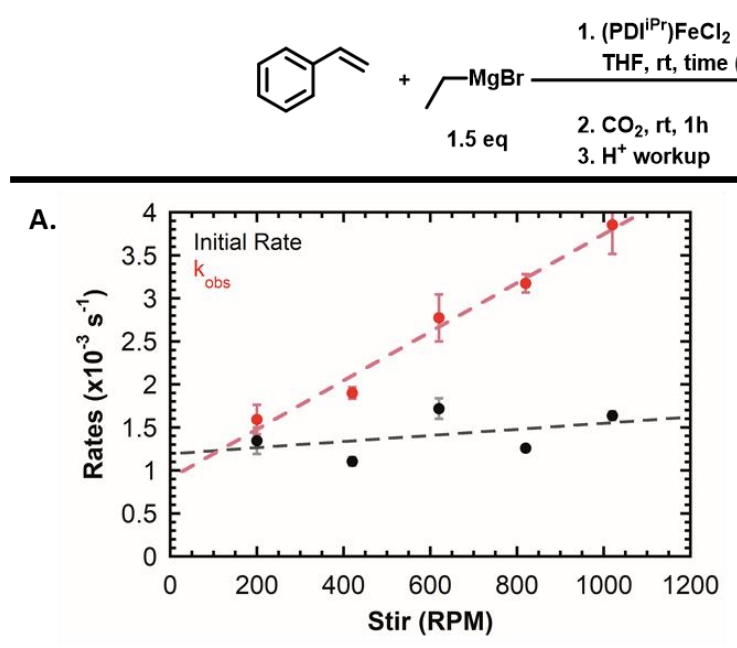

C.

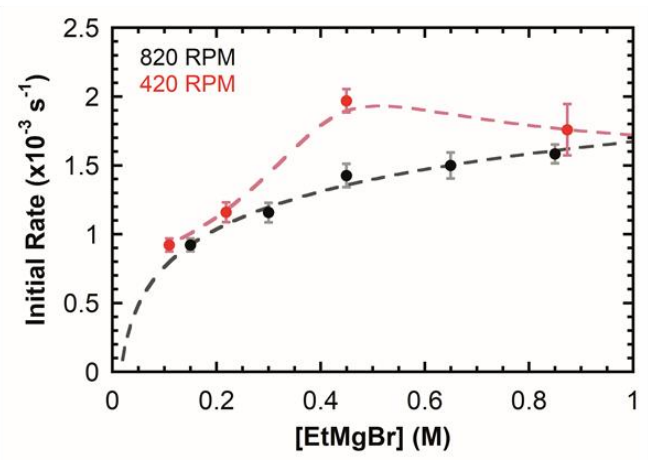

B.

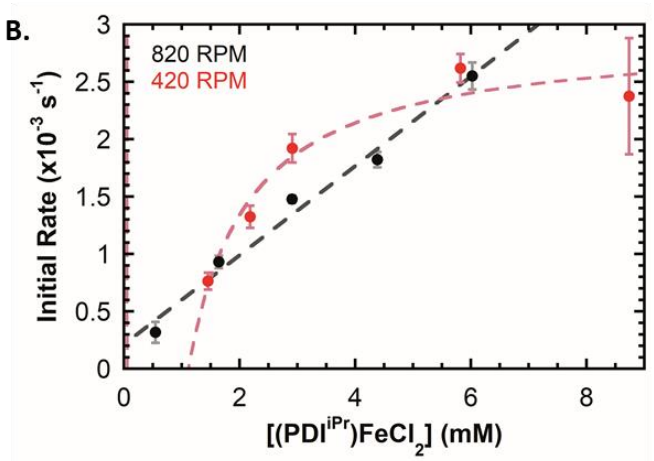

D.

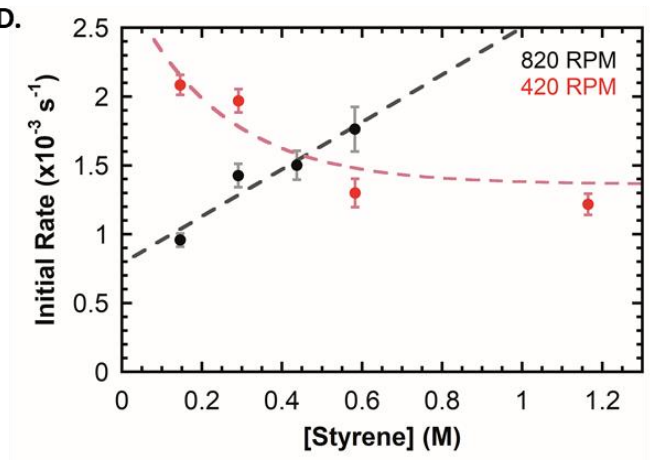

Figure 4.3: Hydromagnesiation using ethyl Grignard. Standard Conditions: (PDIPr) $\mathrm{FeCl}_{2}(1 \mathrm{~mol} \%), \mathrm{S}(1.0 \mathrm{eq}), \mathrm{GEt}^{\mathrm{Et}}$ (1.5 eq), THF, Ar (1 atm), rt, 1 h. Quench: 1) $\mathrm{CO}_{2}$ (dry), rt, 1h; 2) $1.0 \mathrm{M} \mathrm{HCl}$, rt, overnight ${ }^{195}$ 


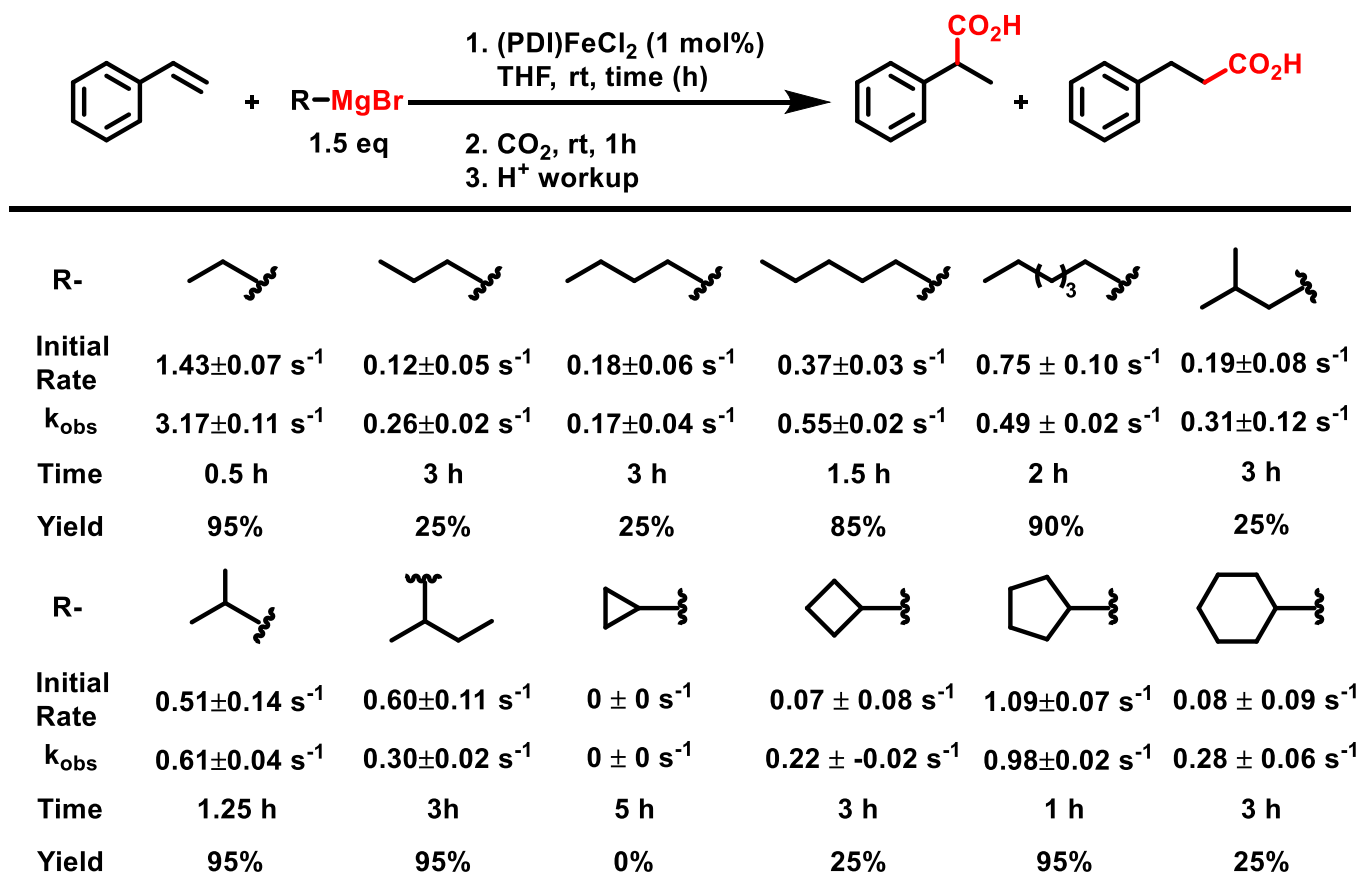

Scheme 4.2: Hydromagnesiation using various Grignards. Conditions: (PD|Pr $) \mathrm{FeCl}_{2}$ (1 mol\%), S (1.0 eq), GX (1.5 eq), THF, Ar (1 atm), rt, 2 h. Quench: 1) $\mathrm{CO}_{2}$ (dry), rt, 1h; 2) $1.0 \mathrm{M} \mathrm{HCl}$, rt, overnight

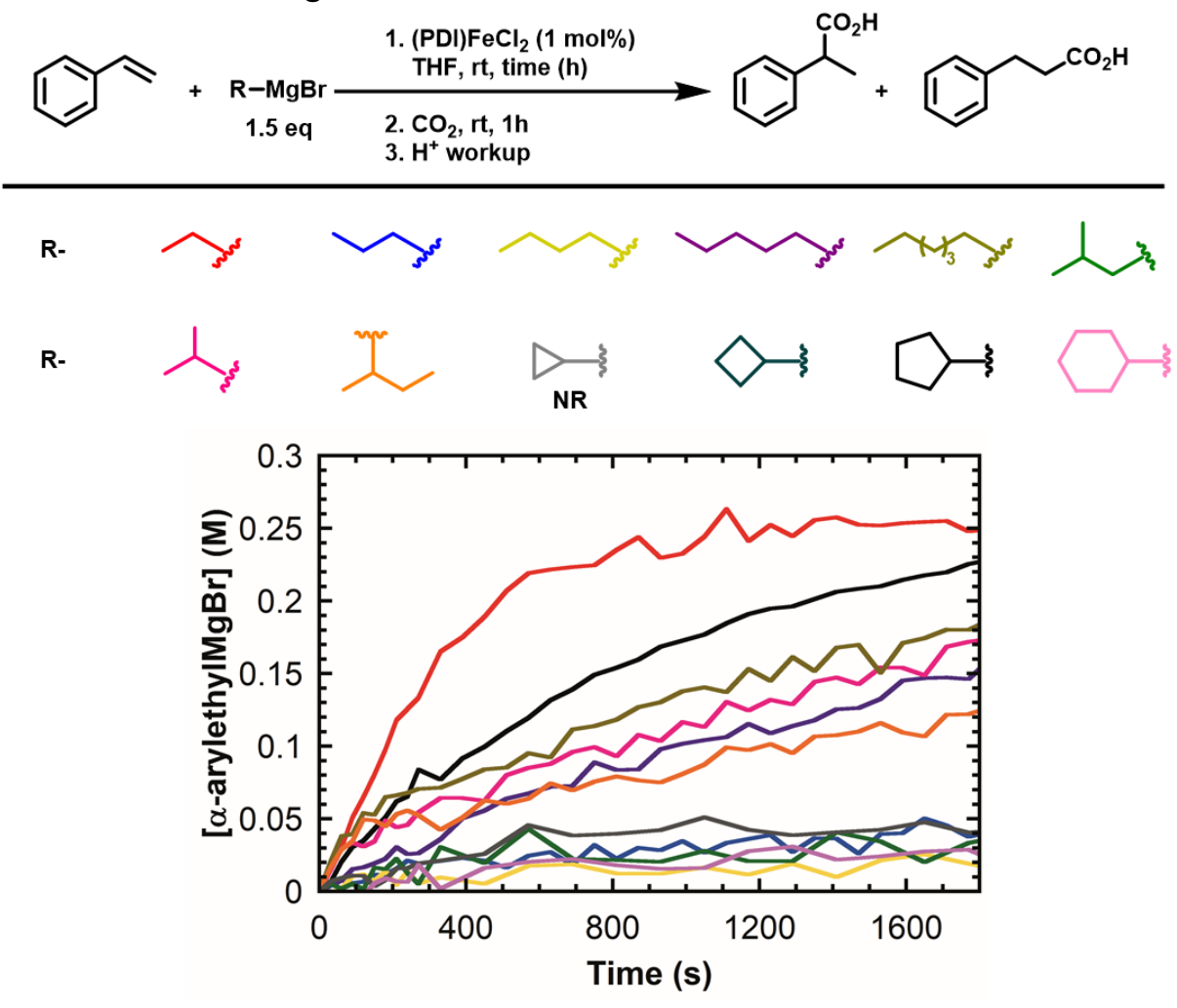

Figure 4.4: Hydromagnesiation with other Grignard substrates. Conditions: (PDIPr) $\mathrm{FeCl}_{2}(1 \mathrm{~mol} \%), \mathrm{S}(1.0 \mathrm{eq}), \mathrm{GX}^{\mathrm{X}}(1.5 \mathrm{eq}), \mathrm{THF}, \operatorname{Ar}(1 \mathrm{~atm}), \mathrm{rt}, 2 \mathrm{~h}$. Quench: 1) $\mathrm{CO}_{2}$ (dry), rt, 1h; 2) $1.0 \mathrm{M} \mathrm{HCl}$, rt, overnight 

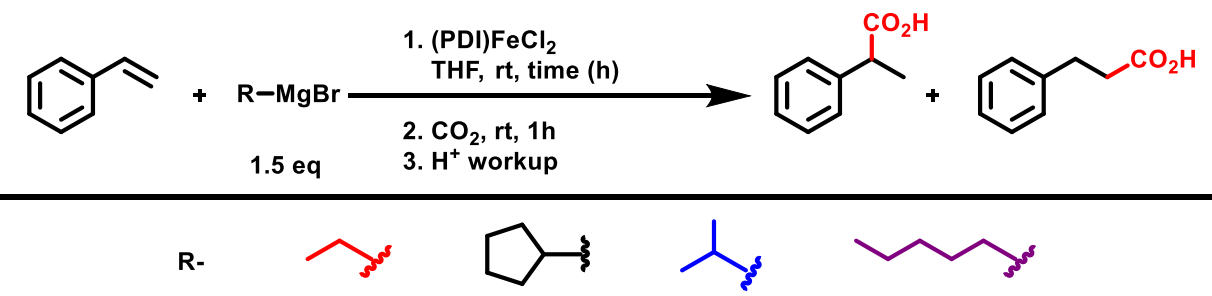

A.

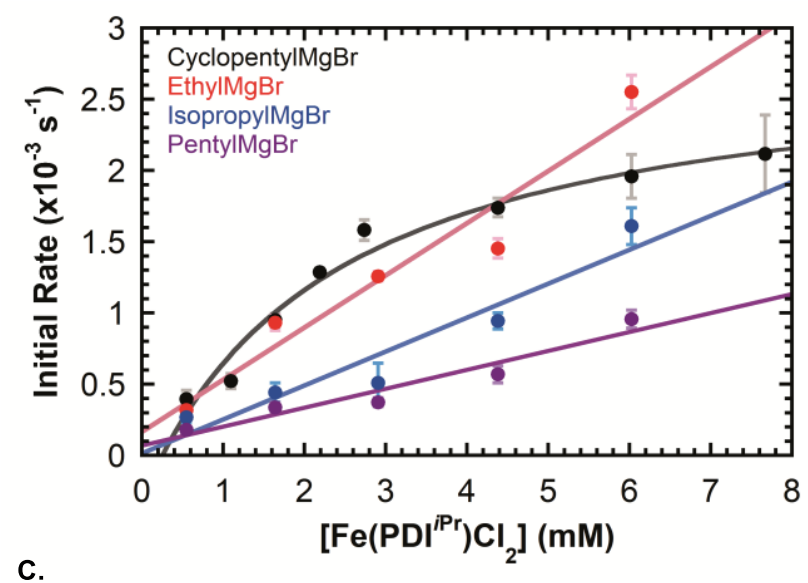

C.

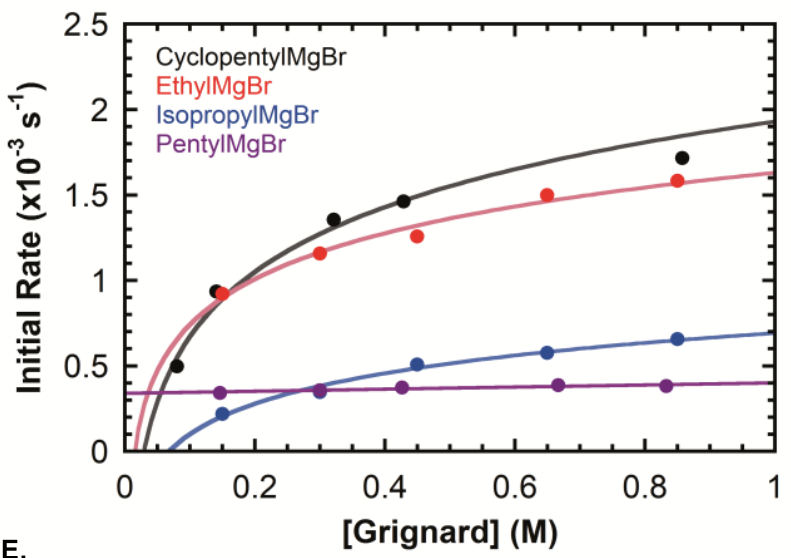

E.

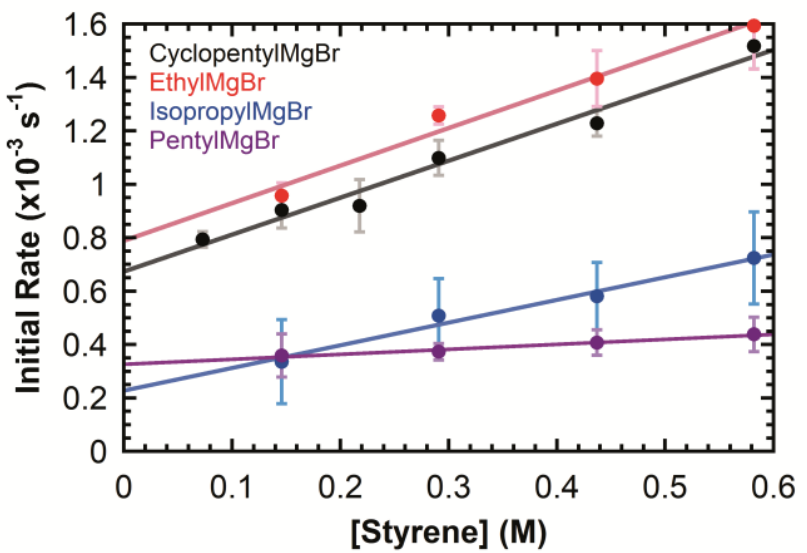

B.

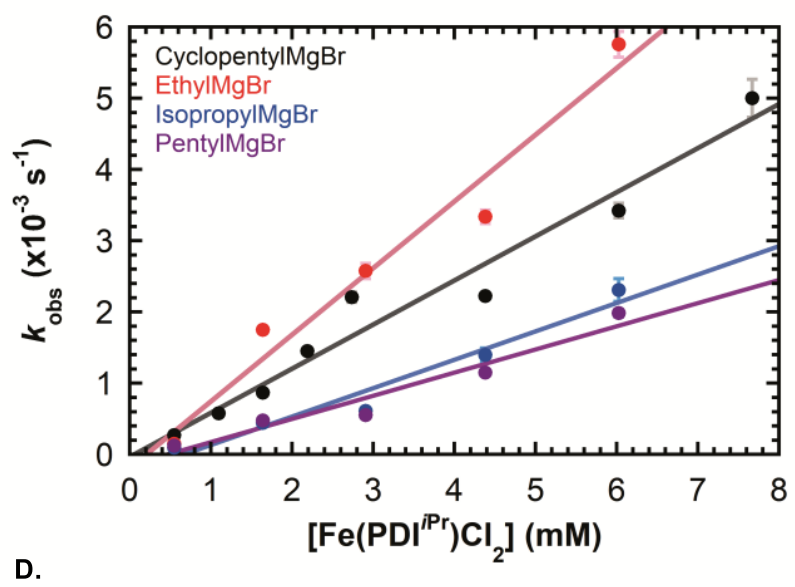

D.
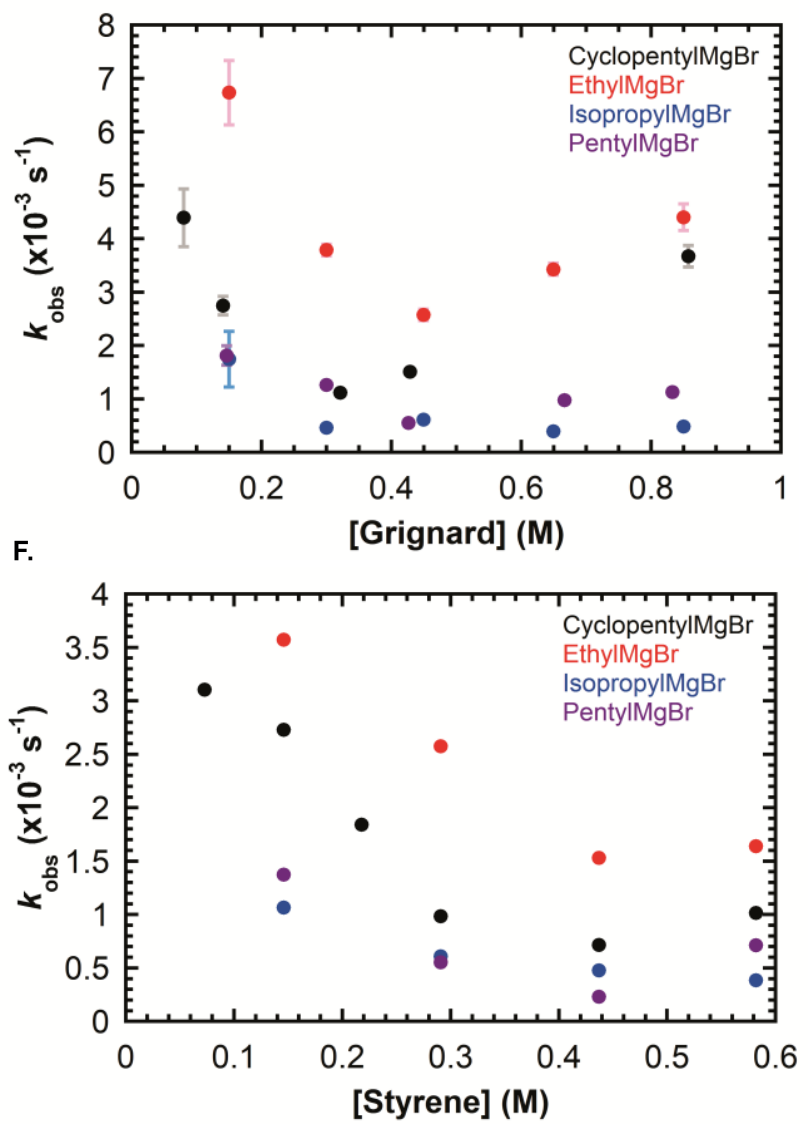

Figure 4.5: Concentration dependencies. Standard Conditions: ( $\mathrm{PD} \mid \mathrm{Pr}) \mathrm{FeCl}_{2}$ (1 mol\%), S (1.0 eq), GX (1.5 eq), THF, Ar (1 atm), rt, 2 h. Quench: 1) $\mathrm{CO}_{2}$ (dry), rt, 1h; 2) $1.0 \mathrm{M} \mathrm{HCl}$, rt, overnight 
Following the determination of the concentration dependencies of $G^{E t}$, a variety of other Grignards were analyzed including those initially studied by Thomas/Neidig. Validating the trends Thomas described, GEt resulted in the highest initial rate and observed rate, Scheme 4.2. 153,170,195 Surprisingly, propyl-, butyl-, cyclobutyl- and cyclohexyl- Grignards resulted in low yields and conversion (Scheme 4.2 and Figure 4.4). This may be the resultant of a few factors: 1) the generation of gaseous propylene/butene may impede coordination of styrene, 2) the unfavorable energetics of cyclobutene may cause for issues with hydride transfer and 3) the steric bulk of cyclohexyl group could encumber styrene coordination. Although isopropylMgBr results in high yields and conversion, the both initial and observed rates were much lower than those of cyclopentylMgBr. Thomas alluded to this behavior, recently. ${ }^{195}$ To determine other concentration dependencies for some of the Grignard reagents that resulted in high conversion and yields, initial rate and observed rate analyses were conducted for ethyl-, isopropyl- and pentylMgBr ( $\mathrm{G}^{\mathrm{Et}}, \mathrm{G}^{\mathrm{iPr}}$ and $\mathrm{G}^{\mathrm{Pent}}$, respectively). Figure 4.5 describes the kinetic analyses.

Upon introducing new Grignards to our catalytic system, new kinetic behavior of the (PDI)-iron catalyst was noticed. Based upon initial rates analysis, for other Grignard reagents the catalyst seems to become a first-order catalyst. This dependence was noted to be similar to the studies by Thomas/Neidig using $G^{E t}$ and $G^{i P r}$. Continuing other concentration dependencies, Grignard and styrene dependencies resulted in similar trends as seen in Chapter 2. COPASI studies comparing G ${ }^{\text {Cyp }}$, GEt, and $G^{\text {iPr }}$ were introduced to discuss changes in the reaction mechanism with respect to other Grignard reagents. Upon using $\mathrm{G}^{\mathrm{iPr}}$ and $\mathrm{G}^{\mathrm{Pent}}$, some of the timecourse began displaying a curve that did not have true exponential character. However, the timecourses when numerically modeled against our standard mechanism, Scheme 2.8, resulted in moderate agreement. Upon the addition of an elementary step to include the formation of the (PDI)Fe(ethyl) ethylene species noted by Thomas/Neidig (Scheme 4.3), the timecourses of the GEt concentration dependencies displayed excellent agreement (Figure 4.6). ${ }^{195}$ The timecourses for the GiPr dependencies resulted in significant agreement (Figure 4.7); however, at low concentrations of Styrene and Grignard if the elementary step resulting 
in the formation of the iron(alkyl) alkene complex is irreversible Figure 4.8. The calculated timecourses introduce the viability of the competition in binding of propylene and styrene to the iron-alkyl species, II. This may also explain the issues with $n$-propylMgBr, the main difference between these substrates is the number of $\beta$-hydrogens. Since isopropylMgBr contains $6 \beta$-hydrogens there is a better chance for hydride transfer when compared to the linear propyl moiety.

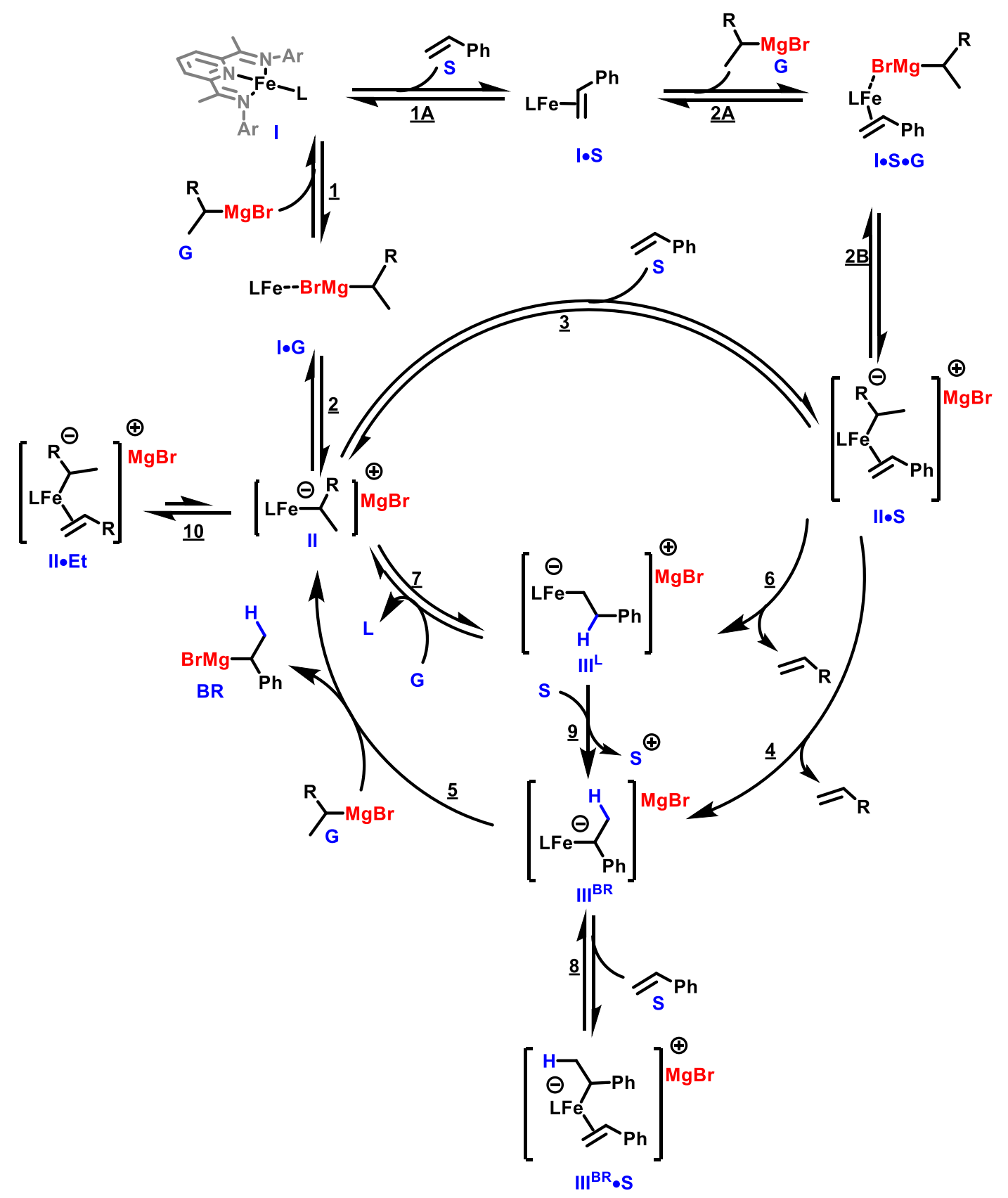

Scheme 4.3: Proposed mechanism of iron-catalyzed hydromagnesiation with ethyl and isopropylMgBr. $\left(L=P D I^{i P r}, L=\right.$ linear isomer, $\left.R=H / M e\right)$ 


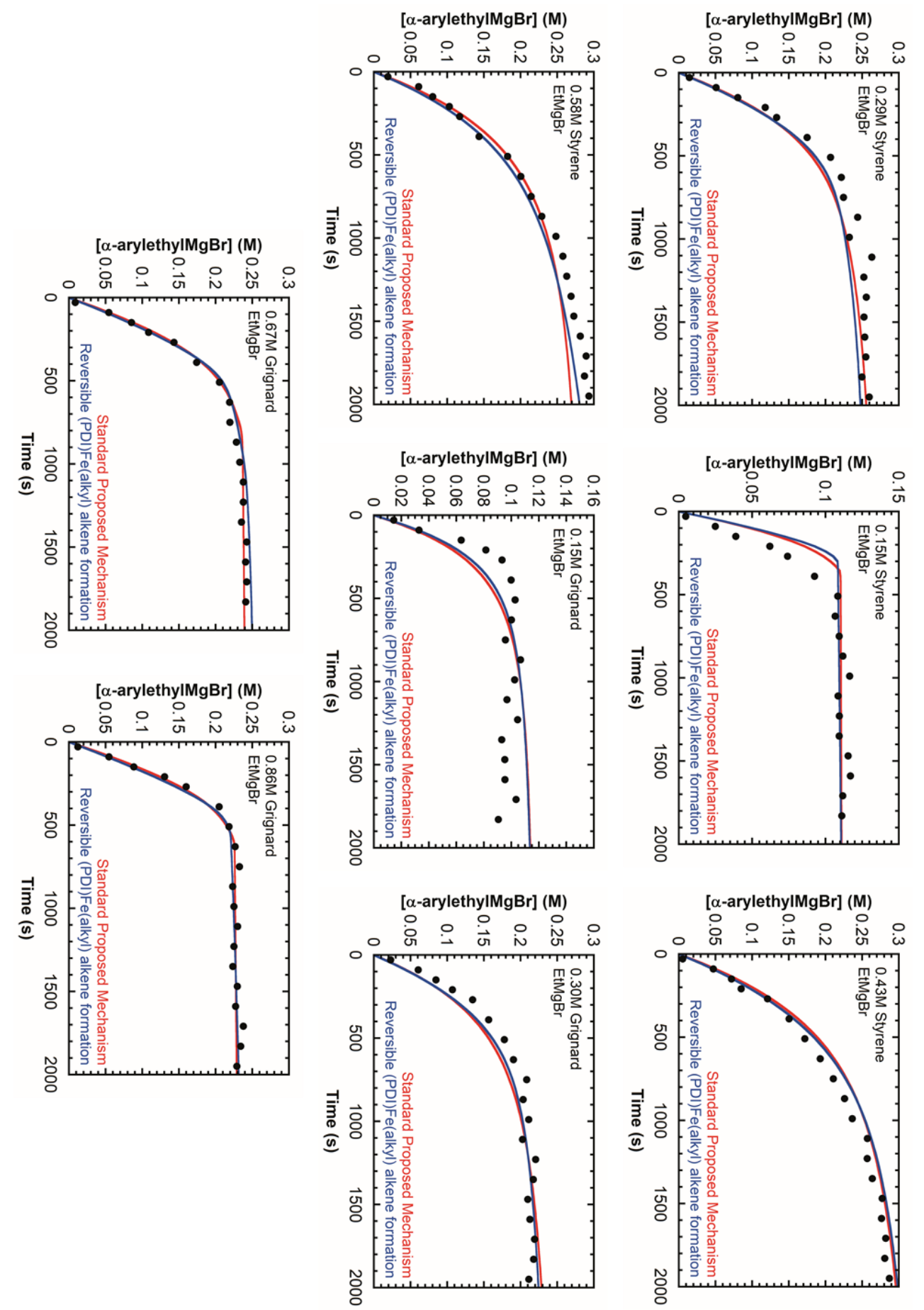

Figure 4.6: COPASI calculations for EthylMgBr. Standard Conditions: (PDIPr) $\mathrm{FeCl}_{2}$ (2.9 mM), S (0.29 M), GEt (0.43 M), THF, Ar (1 atm), rt, 820 RPM, 1 h. Quench: 1) $\mathrm{CO}_{2}$ (dry), rt, 1h; 2) $1.0 \mathrm{M} \mathrm{HCl}$, rt, overnight. Cohen's kappa: $\mathrm{G}^{\mathrm{Et}}$ std mech: moderate agreement $\kappa_{\text {std }}=\frac{(75 \%)-(50 \%)}{1-50 \%}=0.5 ; \mathrm{Fe}($ alkyl) alkene form (Scheme 4.3, rev step 10): substantial agreement $\kappa_{\text {rev } 10}=\frac{(90 \%)-(50 \%)}{1-50 \%}=0.80$. 


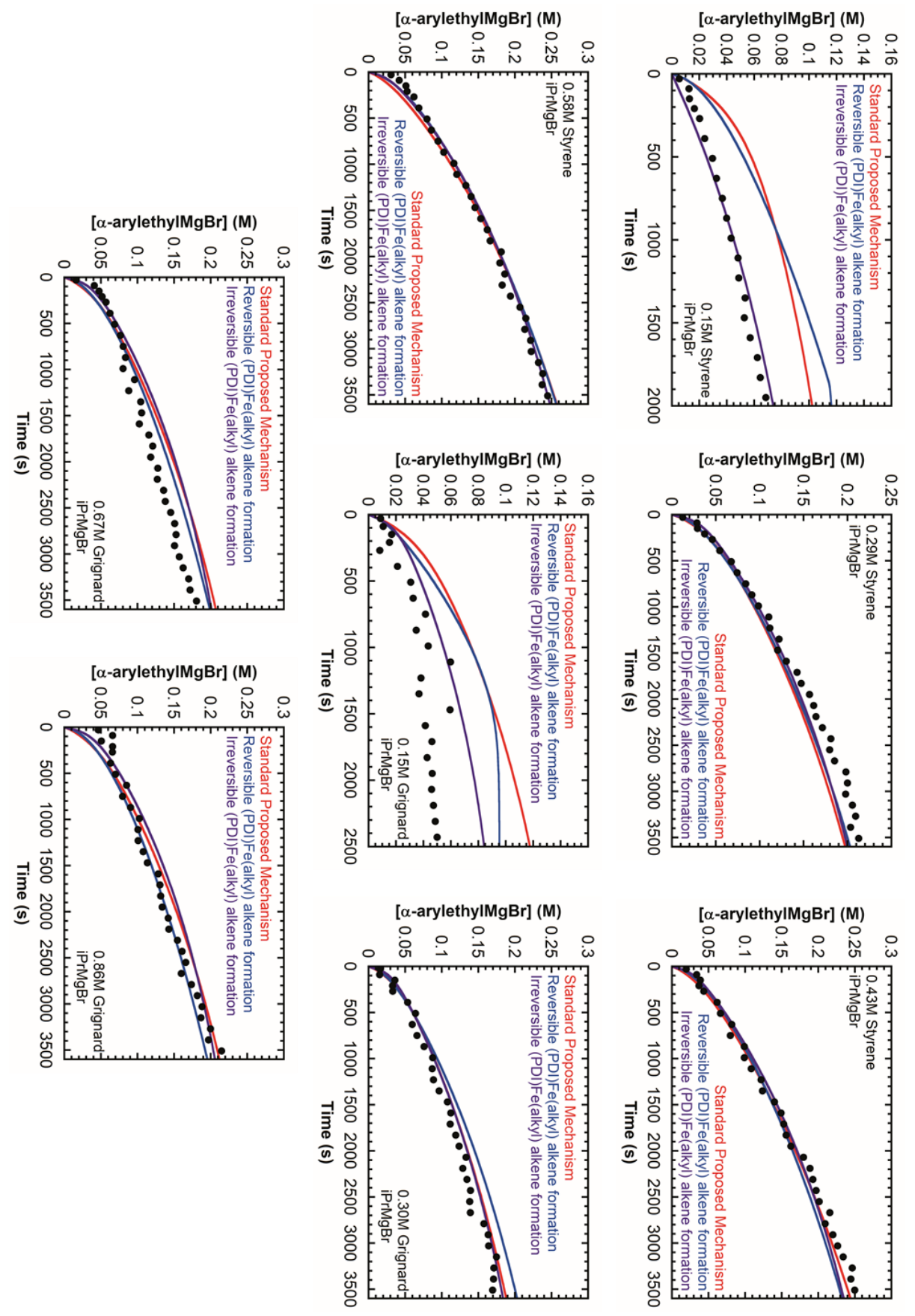

Figure 4.7: COPASI calculations for EthylMgBr. Standard Conditions: (PDIPr) $\mathrm{FeCl}_{2}$ (2.9 mM), S (0.29 M), GiPr (0.43 M), THF, Ar (1 atm), rt, 820 RPM, 1 h. Quench: 1) $\mathrm{CO}_{2}$ (dry), rt, $1 \mathrm{~h}$; 2) $1.0 \mathrm{M} \mathrm{HCl}$, rt, overnight Cohen's kappa: GiPr: minimal agreement $\kappa_{s t d}=\frac{(35 \%)-(50 \%)}{1-50 \%}=-0.25 ; \mathrm{Fe}($ alkyl) alkene form (Scheme 4.3, rev step 10): fair agreement $\kappa_{\text {rev } 10}=\frac{(62 \%)-(50 \%)}{1-50 \%}=0.25 ; \mathrm{Fe}($ alkyl) alkene form (Scheme 4.3, irr step 10): excellent agreement $\kappa_{\text {irrev } 10}=\frac{(90 \%)-(50 \%)}{1-50 \%}=0.80$. 
A.

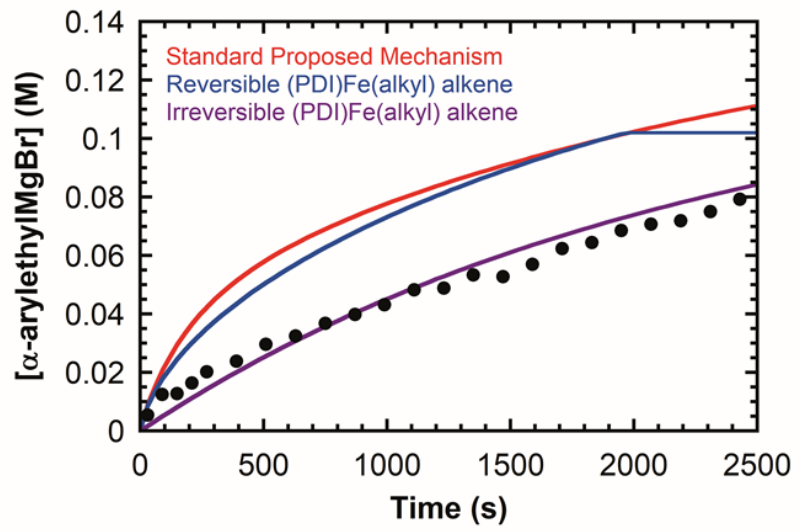

B.

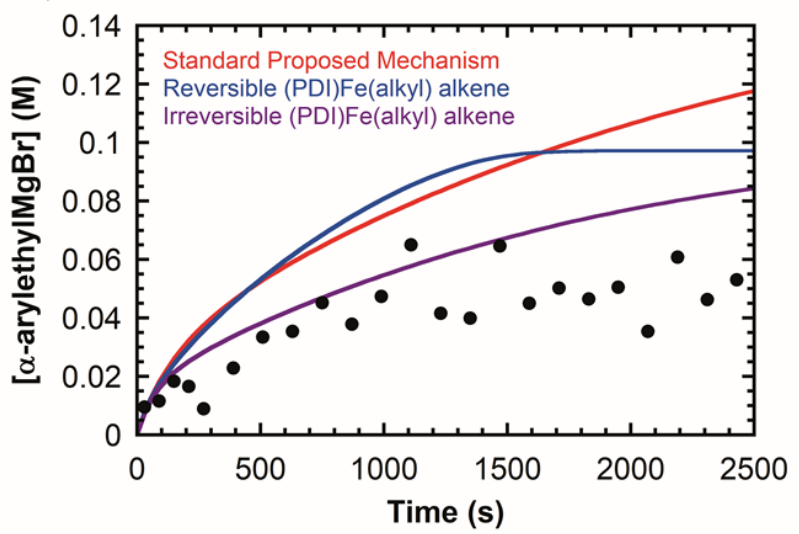

Figure 4.8: COPASI calculations low [styrene] and [Grignard]. Conditions: A) (PDIPr) FeCl2 (2.9 mM), S (0.15 M), GiPr (0.43 M), THF, Ar (1 atm), rt, 2 h. Quench: 1) $\mathrm{CO}_{2}$ (dry), rt, $1 \mathrm{~h}$; 2) $1.0 \mathrm{M} \mathrm{HCl}$, rt, overnight; B) (PDliPr)FeCl2 $(2.9 \mathrm{mM}), \mathrm{S}(0.29 \mathrm{M})$, $\operatorname{Girr}^{\mathrm{iPr}}(0.15 \mathrm{M}), \mathrm{THF}, \operatorname{Ar}(1 \mathrm{~atm}), \mathrm{rt}, 2 \mathrm{~h}$. Quench: 1) $\mathrm{CO}_{2}$ (dry), rt, $1 \mathrm{~h}$; 2) $1.0 \mathrm{M} \mathrm{HCl}$, rt, overnight

\subsection{Conclusions}

The study of Grignard derivatives for hydromagnesiation has been developed. As seen in previous studies by Thomas/Greenhalgh/Neidig, the effect of Grignard is structure dependent. The ability of hydride transfer for each Grignard designates whether hydromagnesiation can occur. With trends similar to Thomas, it was surprising that isopropylMgBr did not hydrometallate as efficiently as ethylMgBr. Following other trends based upon the work of $\mathrm{Xi}$ and others using titanium with isopropylMgBr, the hydrometallation rates and generation of formal titanium-hydride species may suggest that the hydride transfer for iron would react similarly. ${ }^{157,184}$ However, within our study we found that this is not the case. The absence of an exponential fit for multiple timecourses and the necessity of the introduction of an elementary step including the iron(alkyl) alkene intermediate (step 10, Scheme 4.3) aided in the identification of certain limitations with regard to Grignard reagents. Such limitations were not limited to 1) ability of the Grignard to undergo hydride transfer, 2) competition of alkene insertion and 3) sterics/electronics of the Grignard alkyl groups. 


\section{Chapter 5. Iron-catalyzed hydromagnesiation: Effects of Catalyst}

\subsection{Introduction}

Substantial studies regarding iron speciation with $\left(\mathrm{PDI}^{\mathrm{PPr}}\right) \mathrm{FeCl}_{2}$ by Thomas and Neidig aided in the determination of a formal iron(0)-ate species over the course of the reaction. ${ }^{195}$ Despite this, their studies neglected to discuss the effects of the ligand. ${ }^{195}$ To develop an analysis which results in a fully developed explanation of all variants of iron-catalyzed hydromagnesiation, the effect of the ligand must be also be expanded upon. Sadly, the literature of non-redox innocence and ligand effect for iron-catalyzed hydrometallation reactions is sparse; therefore, to begin discussions of the ligand effects of (PDI)ironcatalyzed polymerization trends may provide a helpful insight. Past mechanistic analyses of (PDI)iron-catalyzed polymerization described the following trend: 1) rate of polymerization increases for di-ortho substituted PDI ligands as steric bulk is reduced and 2) the rate of oligomerization increases for mono-ortho substituted PDI ligands as the steric bulk of the ortho-group is reduced, Scheme 5.1. ${ }^{210,211}$ If one bases the effect of PDI ligand for polymerization then two trends could be noted: 1) the decrease in steric bulk could increase the overall rate of hydromagnesiation and 2) the change in reactivity from polymerization to oligomerization might cause a change in mechanism based upon ligand substitution.

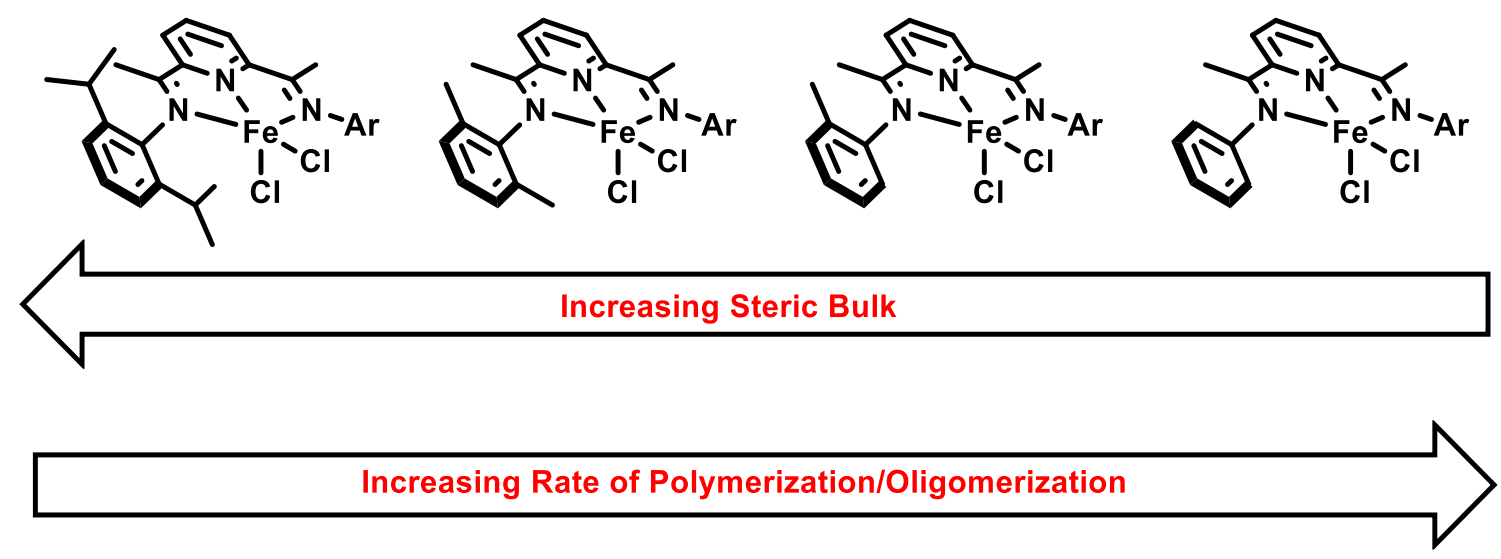

Scheme 5.1: Effect of (PDI)iron-catalysts for polymerization. ${ }^{210,211}$

Full in situ infrared mechanistic studies of (PDI)iron-catalyzed hydromagnesiation then add the final piece needed for the develop the process of iron-catalyzed transfer hydrometallation reactions. Within this chapter, the effect of ligand as well as any -80- 
limitations that arise from a change in steric bulk are discussed. The following ligands will be complexed with anhydrous iron(II) chloride as the precatalysts: 2,6-bis[1-(2,6diisopropylphenylimino)ethyl] pyridine (PDI $\left.{ }^{\mathrm{PP}}\right)$, 2,6-bis[1-(2,6-dimethylphenylimino)ethyl] pyridine (PDIMe), 2,6-bis[1-(2-methylphenyimino)ethyl] pyridine (PDI $\left.{ }^{2-M e}\right)$ and 2,6-bis[1(phenyimino)ethyl] pyridine $\left(\mathrm{PDI}^{\mathrm{H}}\right)$. These ligand motifs were among the most common used for olefin polymerization catalysis of iron and cobalt systems and provide a point of comparison for this iron-catalyzed hydromagnesiation process.

\subsection{Results}
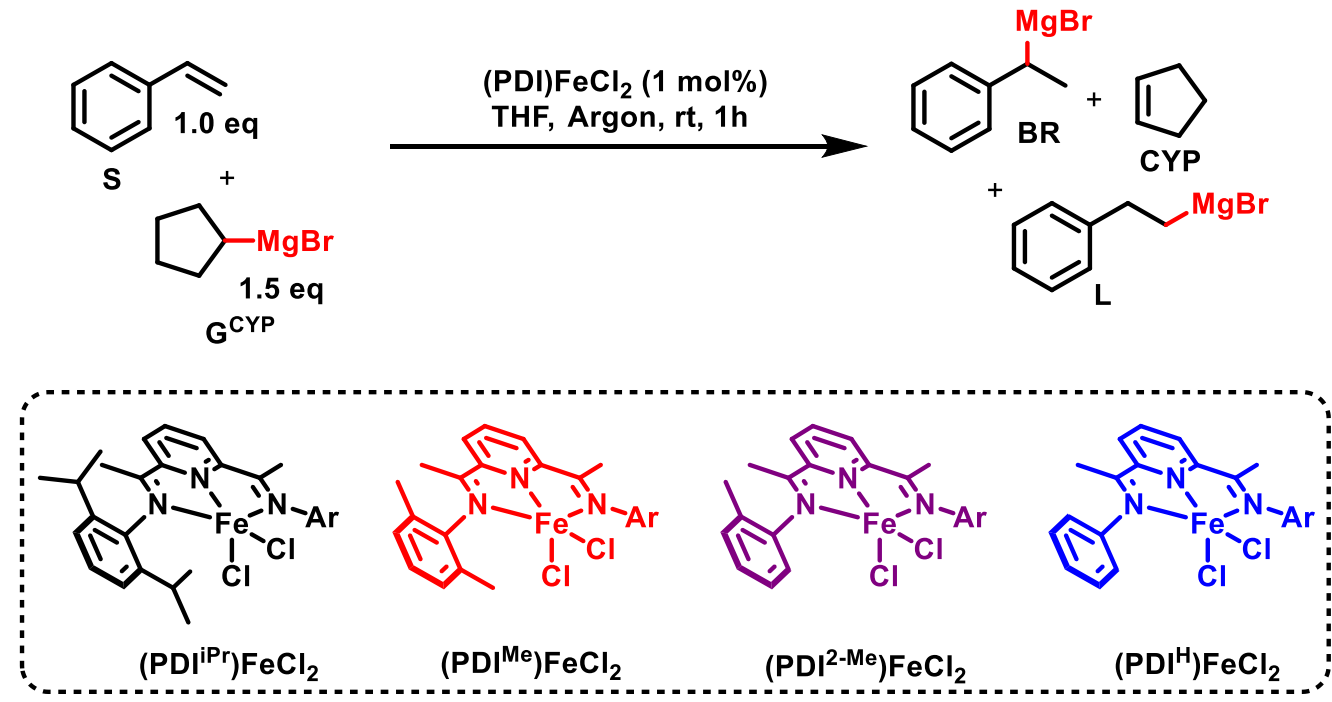

Scheme 5.2: Iron-catalyzed hydromagnesiation with cyclopentylMgBr, GCYP. Conditions: anhydrous (PDI ${ }^{\mathrm{X}} \mathrm{FeCl}_{2}(1 \mathrm{~mol} \%), \mathrm{S}(1.0 \mathrm{eq}, 0.87 \mathrm{mmol}, 0.3 \mathrm{M})$, GCYP (1.5 eq, $1.3 \mathrm{mmol}, 0.43 \mathrm{M}$ ), THF, argon (1 atm), rt, $1 \mathrm{~h}$. Quench: 1) $\mathrm{CO}_{2}$ (dry), rt, $1 \mathrm{~h}$; 2) $1.0 \mathrm{M} \mathrm{HCl}$, rt, overnight. 

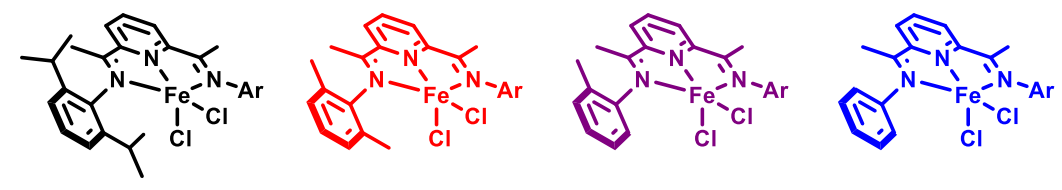

A.

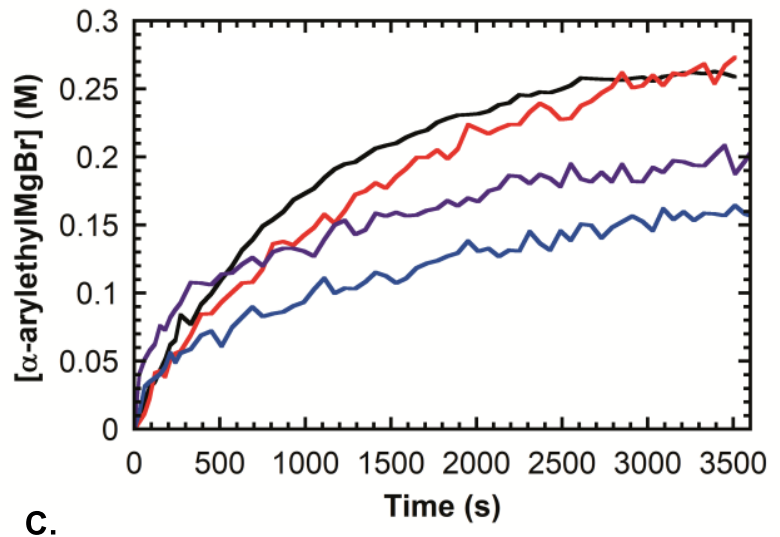

B.
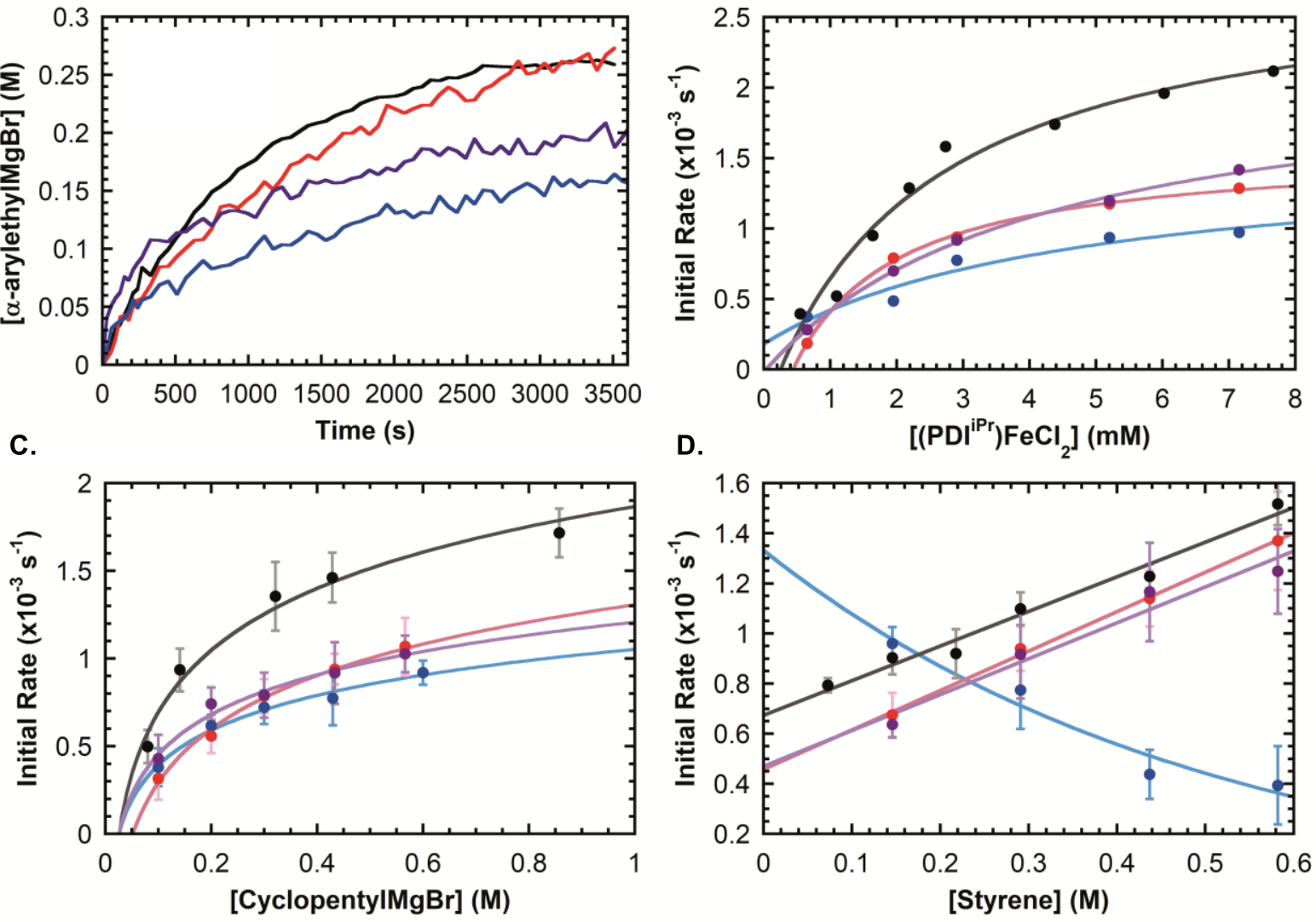

Figure 5.1: A) Timecourse of standard reaction concentrations for each iron catalyst; B-D) Concentration dependencies. Standard Conditions: (PDIPr)FeCl 2 (1 mol\%), S (1.0 eq), G ${ }^{C Y P}$ (1.5 eq), THF, Ar (1 atm), rt, 2 h. Quench: 1) $\mathrm{CO}_{2}$ (dry), rt, 1h; 2) $1.0 \mathrm{M}$ $\mathrm{HCl}, \mathrm{rt}$, overnight

The iron dichloride precatalysts described in Scheme 5.1 were synthesized according to literature. ${ }^{202,210-212}$ The iron-complexes bearing ortho-substituents (PDI ${ }^{\mathrm{PPr}}, \mathrm{PDI}^{\mathrm{Me}}, \mathrm{PDI}^{2-}$ $\mathrm{Me})$ were royal blue in color while the phenyl-PDI $\left(\mathrm{PDI}^{\mathrm{H}}\right)$ was a deep purple. The in situ infrared analysis described in Chapter 2 was used to determine the initial rates for each catalyst in the system, Scheme 5.2. Under standard conditions, the reaction timecourses for each precatalyst did not overlap suggesting that each catalyst system may display different kinetic dependencies (Figure 5.1A). To begin the initial rates analysis of the precatalysts, the catalyst concentration dependence of each still remarkably displays a 
hyperbolic fit, Figure 5.1B. The initial saturation dependence of Grignard still is representative of the initial rates analysis (Figure 5.1C). However, new trends resulted from the styrene concentration dependencies. Figure 5.1D illustrates a change in reactivity, upon loss of steric bulk the first-order non-zero intercept kinetic behavior became inhibitory for the $\left(\mathrm{PDI}^{\mathrm{H}}\right) \mathrm{FeCl}_{2}$ precatalyst. Unfortunately, the observed rates for the catalyst systems resulted in a timecourse that was not exponential in nature, therefore assuming an exponential to calculate the $k_{o b s}$ is not viable under these conditions. Regrettably, we could not glean any information from the observed rate analyses. However, the trends noted in all previous chapters for observed rates were used as a starting point for further analysis of this chapter. Then, the same numeric modeling within COPASI was employed starting from the standard mechanism, Scheme 5.3.

Upon applying the evolutionary programming parameter estimation for each precatalyst and LSODA timecourse determination, the proposed mechanism of ironcatalyzed hydromagnesiation (Scheme 5.3) resulted in only moderate agreement (Figures 5.2 and 5.3). Therefore, more mechanistic modeling was needed. With Thomas and Neidig's characterization of the off-cycle ( $\eta^{2}$-styrene $)_{3} \mathrm{Fe}^{0}$ (benzyl) anion intermediate in mind, the generation of such an intermediate could be a result from III ${ }^{\mathrm{BR}} \cdot \mathrm{S}$ irreversible ligand dissociation and reactivity with multiple equivalents of styrene. ${ }^{195}$ This intermediate was determined by Thomas/Neidig to be catalytically competent. The introduction of elementary steps to include the generation of $\left(\eta^{2}\right.$-styrene) ${ }_{3} \mathrm{Fe}^{0}$ (benzyl) anion (IV) and its reactivity was introduced into the mechanism (Scheme 5.4). 


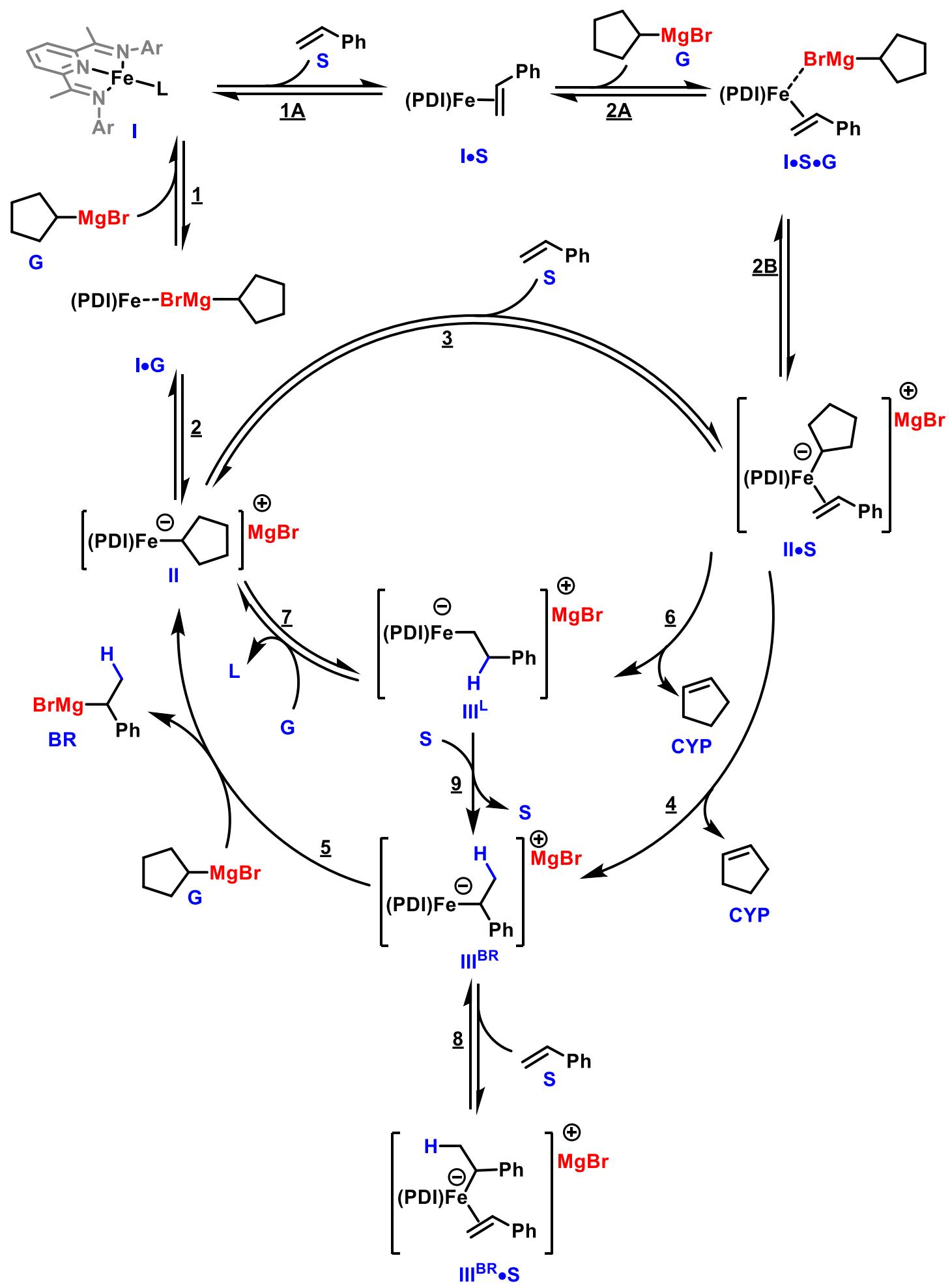

Scheme 5.3: Proposed mechanism of iron-catalyzed hydromagnesiation with a variety of precatalysts. $(L=$ linear isomer) 


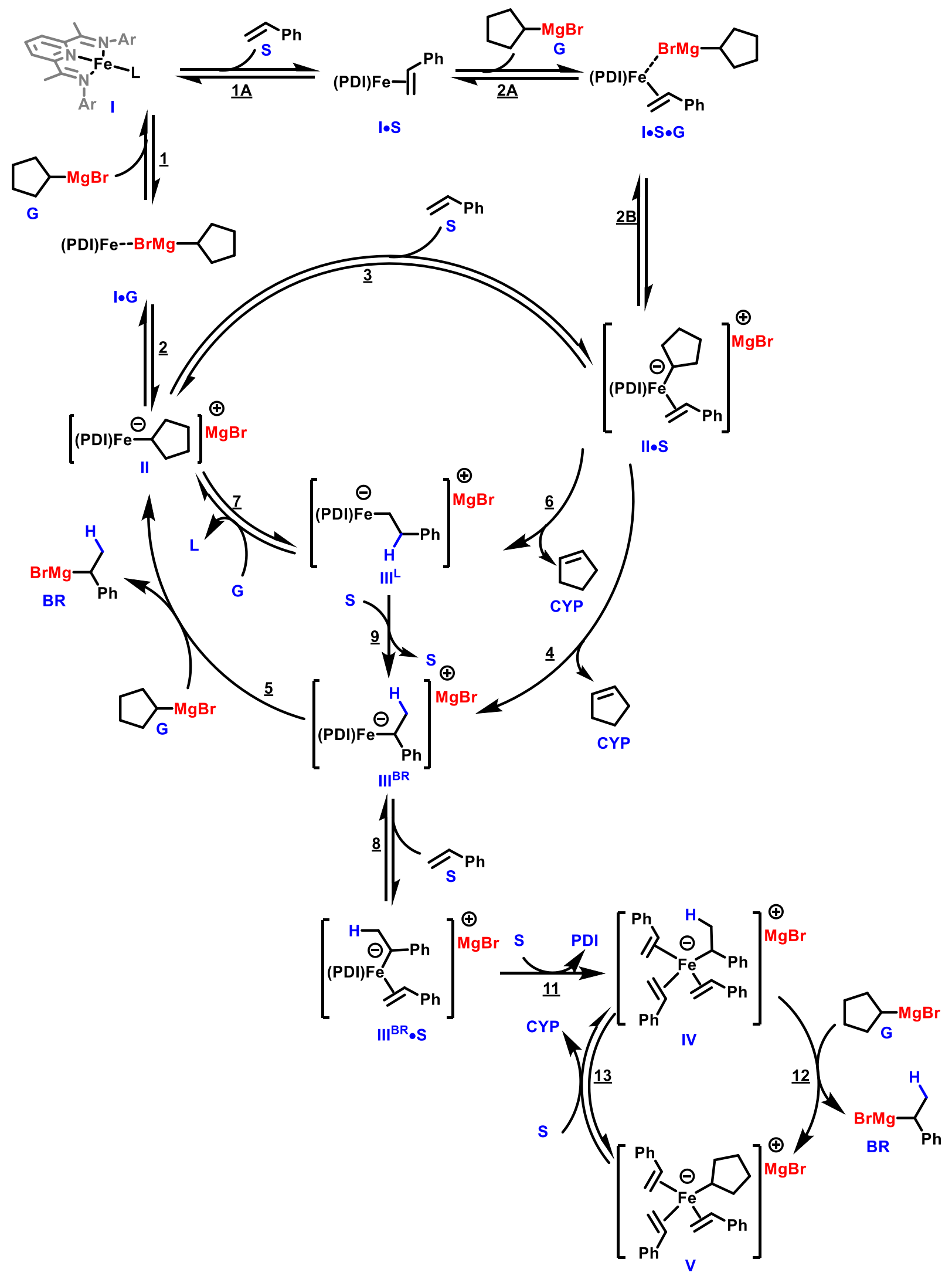

Scheme 5.4: Proposed mechanism with a variety of precatalysts $(L=$ linear isomer) 


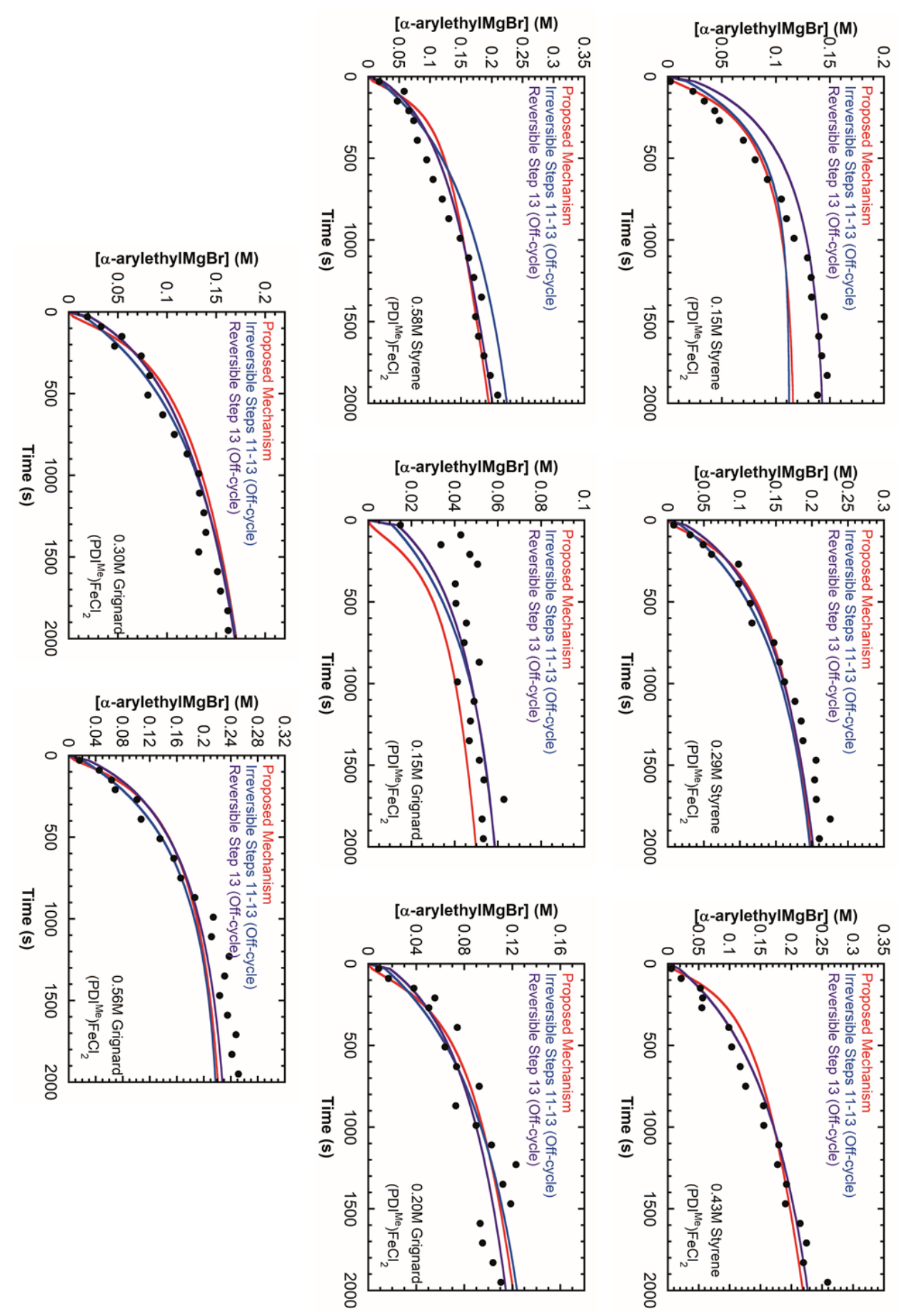

Figure 5.2: COPASI calculations for $\left(\mathrm{PDI}{ }^{\mathrm{Me}}\right) \mathrm{FeCl}_{2}$. Standard Conditions: $\left(\mathrm{PDI}{ }^{\mathrm{Me}}\right) \mathrm{FeCl}_{2}$ (2.9 mM), S (0.29 M), GCYP (0.43 M), THF, Ar (1 atm), rt, 1 h. Quench: 1) $\mathrm{CO}_{2}$ (dry), rt, $1 \mathrm{~h}$; 2) $1.0 \mathrm{M} \mathrm{HCl}$, rt, overnight Cohen's kappa: PDIMe: minimal agreement $\kappa_{\text {std }}=$ $\frac{(50 \%)-(50 \%)}{1-50 \%}=0.0$; Off-cycle (Scheme 5.4, irr step 13): fair agreement $\kappa_{\text {rev } 10}=$ $\frac{(62 \%)-(50 \%)}{1-50 \%}=0.25$; Off-cycle (Scheme 5.4 , rev step 13): substantial agreement $\kappa_{\text {irrev } 10}=\frac{(85 \%)-(50 \%)}{1-50 \%}=0.70$. 


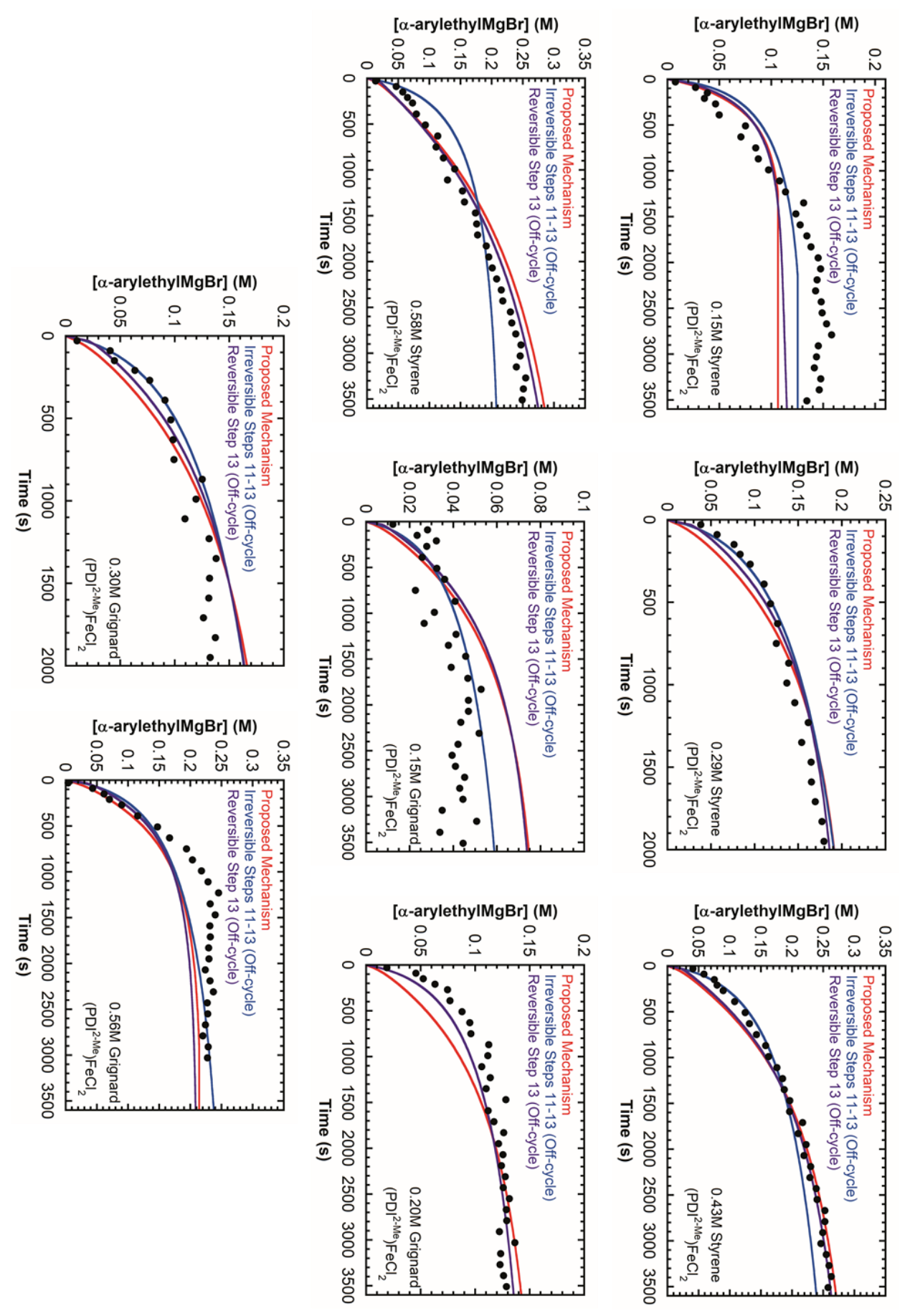

Figure 5.3: COPASI calculations for $\left(\mathrm{PDI}^{2-\mathrm{Me}}\right) \mathrm{FeCl}_{2}$. Standard Conditions: $\left(\mathrm{PDI}^{2-}\right.$ $\left.{ }^{\mathrm{Me}}\right) \mathrm{FeCl}_{2}(2.9 \mathrm{mM}), \mathrm{S}(0.29 \mathrm{M}), \mathrm{G}^{\mathrm{CYP}}(0.43 \mathrm{M}), \mathrm{THF}, \operatorname{Ar}(1 \mathrm{~atm})$, rt, $1 \mathrm{~h}$. Quench: 1) $\mathrm{CO}_{2}$ (dry), rt, 1h; 2) $1.0 \mathrm{M} \mathrm{HCl}$, rt, overnight Cohen's kappa: $\mathrm{PDI}^{2-\mathrm{Me}}$ : minimal agreement $\kappa_{\text {std }}=\frac{(50 \%)-(50 \%)}{1-50 \%}=0.0 ;$ Off-cycle (Scheme 5.4, irr step 13): fair agreement $\kappa_{\text {rev } 10}=$ $\frac{(62 \%)-(50 \%)}{1-50 \%}=0.25$; Off-cycle (Scheme 5.4, rev step 13): moderate agreement $\kappa_{\text {irrev } 10}=\frac{(75 \%)-(50 \%)}{1-50 \%}=0.50$. 
Upon adding two more elementary steps, the timecourse agreement increases assuming the steps are irreversible (Scheme 5.4, Steps 12-13 and Figures 5.2-3). Although the agreement increased, the timecourse data when the [Grignard] is 3.0+ equiv. compared to [styrene] the product concentration increases steadily in a nonexponential fashion at latter time points. This phenomenon was not reflected within the numeric modelling in conditions that do not include steps 11-13 (Generation of IV, transmetallation and styrene coordination) or when all three steps are irreversible. Upon changing the irreversibility of styrene coordination (Step 13), the reversible reaction gives better overall agreement and displays the increase in product over time (Figures 5.2-3 Purple Trend).

The overall trend in the effect of iron-catalyst for hydromagnesiation is opposite of that of polymerization catalysis. This inversion in trends can be attributed to the probable generation of $\left(\eta^{2}\right.$-styrene ${ }_{3} \mathrm{Fe}^{0}$ (benzyl) anion, IV, which is catalytically competent as evidenced by Thomas. ${ }^{195}$ The introduction of this off-cycle species IV and a secondary cycle that generates product from IV, through ( $\eta^{2}$-styrene) ${ }_{3} \mathrm{Fe}^{0}$ (cyclopentyl) anion, $\mathrm{V}$, may be generated faster upon decreasing the ligand sterics. This may also be evidenced by the absence of an exponential timecourse fitting for less sterically bulky ligands.

\subsection{Conclusions}

The identification of the reaction mechanism for iron-catalyzed hydromagnesiation has allowed for further development within this class of reductive hydrofunctionalization. The expansion of iron catalysts used for hydromagnesiation introduced a new batch of limitations that were not expected when comparing the trends in (PDI)iron-catalyzed polymerization. Namely, the reduction of steric bulk of the PDI ligand increased the rate of polymerization/oligomerization; however, reducing the steric bulk of the ligand in the case of iron-catalyzed hydromagnesiation introduces a non-exponential timecourse fit and generates the catalytically competent $\left(\eta^{2} \text {-styrene }\right)_{3} \mathrm{Fe}^{0}$ (benzyl) anion species characterized by Thomas which then can transmetallate to generate product through an off-cycle catalytic cycle. 


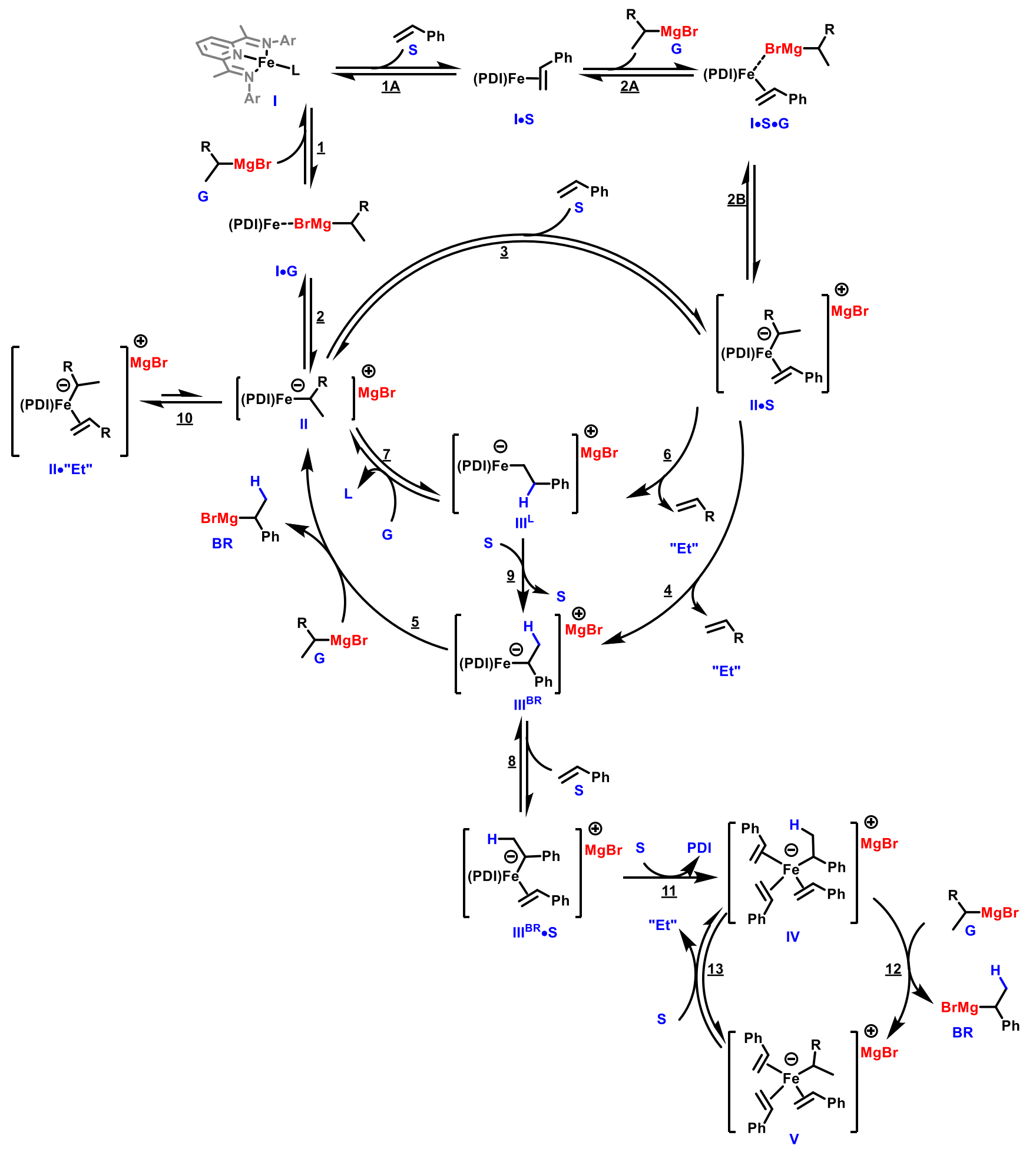

Scheme F.1: Proposed mechanism ( $L=$ linear isomer, $R=H / M e / C y c l o p e n t y l)$ 
To conclude Scheme F.1 depicts a concise mechanism consisting of the combined 16 elementary steps of hydromagnesiation. A formal (PDI)iron(0) species is generated by reacting the iron(II) precatalyst with $>20$ equiv. of a Grignard reagent $\left(G^{X}\right) .{ }^{95,195,195,202-204}$ This formal iron( 0 ) species I, ligated by alkene ("Et") or solvent (THF), enters the catalytic cycle through competing pathways of ligand substitution and catalyst activation. Pathway 1 occurs under limiting styrene conditions wherein I reacts with an equivalent of Grignard reagent to form first an adduct I•G which enters the catalytic cycle as iron-alkyl II. The second pathway, ligand substitution with styrene (S) yields $1 \cdot S$ that subsequently undergoes transmetallation and enters the catalytic cycle as styrene-bound iron-alkyl Il•S. Intermediate $\| \cdot S$ undergoes competing 2,1- and 1,2-hydride transfer pathways to yield iron-benzyl, IIIBR, and iron-homobenzyl, IIIL. Consistent with the observations made with cyclopentene and other disubstituted alkenes, steps 4, 6 and 9 were determined numerically to be irreversible. ${ }^{153,170,195}$ Transmetallation of IIIBR with $\mathrm{G}^{\mathrm{X}}$ is favored over IIIL. However, in the case of $p$-methoxystyrene $\left(S^{\mathrm{OMe}}\right)$, the calculated rates for the generation of $\mathrm{II}^{\mathrm{BR}}$ and $\mathrm{II} \mathrm{L}^{\mathrm{L}}$ are nearly $1: 1$. The reversibility of transmetallation of $\mathrm{II}^{\mathrm{L}}$ (step 7) leads to the irreversible isomerization of $\mathrm{II}^{\mathrm{L}}$ to $\mathrm{II}^{\mathrm{BR}}$ in the presence of excess styrene. The irreversibility of Step 5, transmetallation of $\mathrm{II}^{\mathrm{BR}}$, is also consistent with previous deuterium studies discussed by Thomas, wherein branched Grignard product does not reenter the catalytic cycle. ${ }^{153,170,195}$ In cases where $G^{X}$ would generate a gaseous alkene, the probability of generating an iron(alkyl) alkene intermediate such as $\| \cdot " \mathrm{Et}$ " is more prevalent kinetically, this type of intermediate is also evidenced by Thomas by $\mathrm{x}$-ray crystallographic characterization. ${ }^{195}$ Finally, $\mathrm{III}{ }^{\mathrm{BR}}$ may react with another equivalent of styrene to form off-cycle alkene adduct IIIRR.S. Such a species would be necessary to access the catalytically competent off-cycle $\left(\eta^{2}\right.$-styrene ${ }_{3} \mathrm{Fe}^{0}($ benzyl) anion intermediate (IV) identified and independently prepared by Thomas and Neidig. ${ }^{195}$ This characterized and catalytically competent iron species was noted to only account for a small percentage of iron speciation, however at later time points the concentration of this type of species increased over the course of the reaction. With ligands that are less sterically bulky this type of intermediate could be more prevalent and generates the branched product (BR) in an off-cycle catalytic cycle. This full mechanistic study now allows for experimental predictability based upon changing substrate or catalyst. 
$-91-$ 


\section{Experimental Appendix I Contents:}

1. General information

2. Comparison of Reactant and Product Infrared Spectra.

3. Experimental Procedures

1. Preparation of 2,6-Bis[1-(2,6-dialkylphenylimino)ethyl]pyridine (PDI)

2. Preparation of $(\mathrm{PDI}) \mathrm{FeCl}_{2}$ catalyst

3. Typical reaction time course via ReactIR

4. IGOR Calculations and signal deconvolution

5. Experimental Dependencies

1. Cyclopentene dependence

2. $\left(\mathrm{PDI}{ }^{\mathrm{Pr}}\right) \mathrm{FeCl}_{2}$ dependence

3. Cyclopentyl Grignard dependence

4. Styrene dependence

5. Styrene derivatives

6. Grignard reagent dependence

7. EthylMgBr (Stir)

8. IsopropylMgBr

9. PentylMgBr

10. Precatalysts

6. COPASI Simulations 
1. General Considerations.

All manipulations were performed using an inert atmosphere glovebox or standard Schlenk technique. Anhydrous THF and 1,4-dioxane was dried on a Glass Contours solvent system prior to use and stored under nitrogen in a glovebox. Styrene and styrene derivatives were used as received from Fisher Scientific. Grignards were either purchased and used as is or synthesized following the procedure in section 3.3. IR spectra were recorded on a Mettler Toledo iC IR 15 instrument using iC IR 7.0 analysis program. The THF solvent spectrum was subtracted from acquired spectra within the analysis program.

2. Comparison of Reactant and Product Infrared Spectra Solution infrared spectra were collected for styrene, cyclopentyl magnesium bromide, 1-phenylethylmagnesium bromide, 2-phenylethylmagnesium bromide and a hydromagnesiation reaction for comparison. Two regions showed production of product, $1590 \mathrm{~cm}^{-1}$ and within the fingerprint region at $760 \mathrm{~cm}^{-1}$. The initial rate of reaction could not be calculated using the fingerprint region owing to a significant shoulder from the starting material and tailing from the generation of product. Therefore, the use of the stretches in the $1600 \mathrm{~cm}^{-1}$ region was used for initial rate calculation. Upon normalizing the acquired spectra for comparison, the generation of the major regioisomer, 1-phenylethylmagnesium bromide, shows a stretch at 1590 $\mathrm{cm}^{-1}$. The linear isomer can also be analyzed within this region with a stretch at 1604 $\mathrm{cm}^{-1}$, Figure E.1 (green trace). Both the branched and linear isomers were quenched with $\mathrm{CO}_{2}$, worked up under acidic conditions and identified to yield the respective aryl propionic acids. Styrene and cyclopentyl magnesium bromide were also compared to the hydromagnesiation reaction spectra, Figure E.1 (black and orange traces, respectively). Styrene has a small stretch at $1577 \mathrm{~cm}^{-1}$. Further signal deconvolution is evident based upon overlap between the linear isomer, branched isomer, and styrene, Figure E.3. 

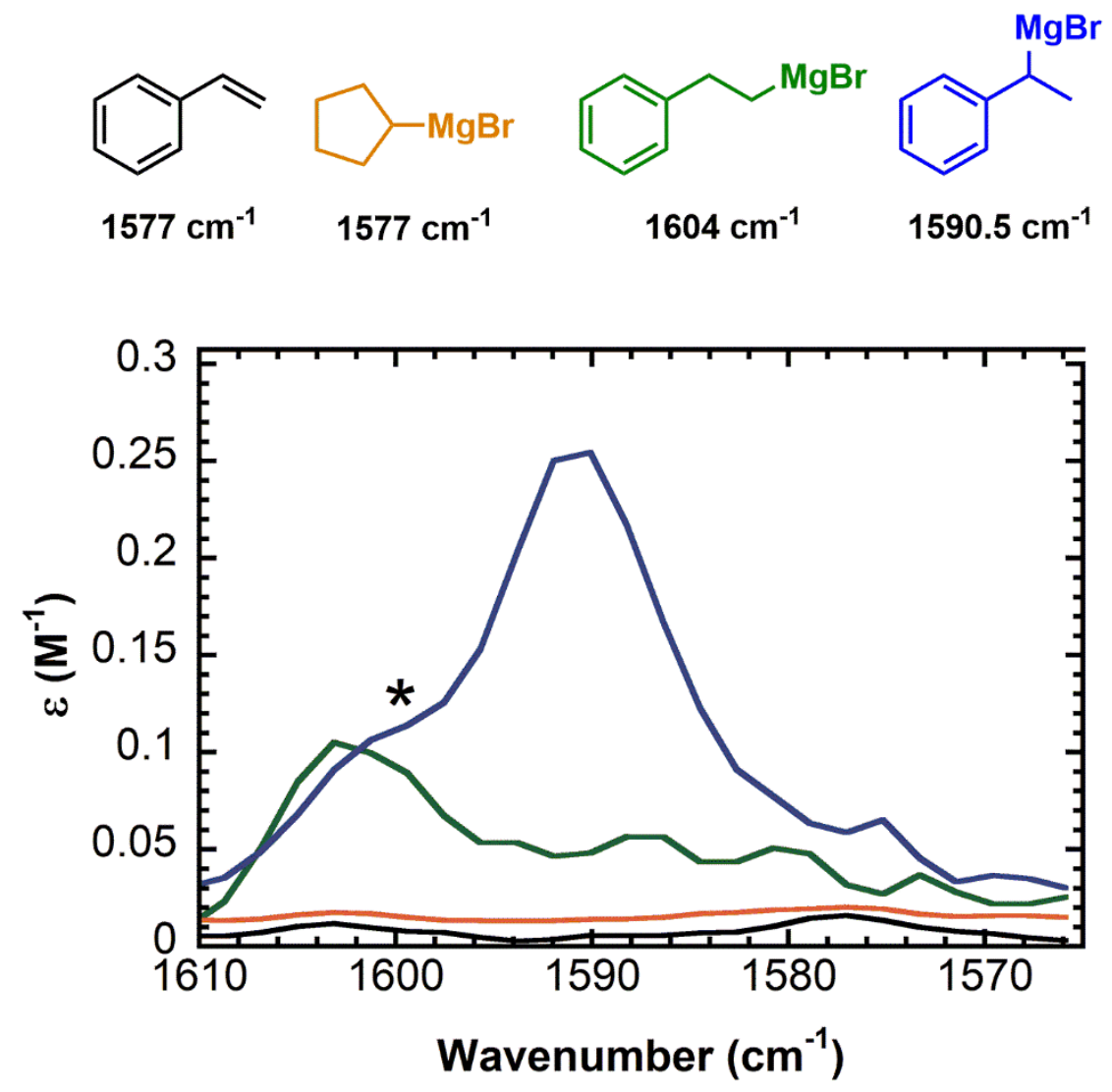

Figure E.1: Frequency analysis of the absorbances of the starting material and the product from Figure 2. Normalized extinction coefficient vs wavenumber. ( ${ }^{*}$ small shoulder acquired from 1-bromo-1-phenylethane overlap)
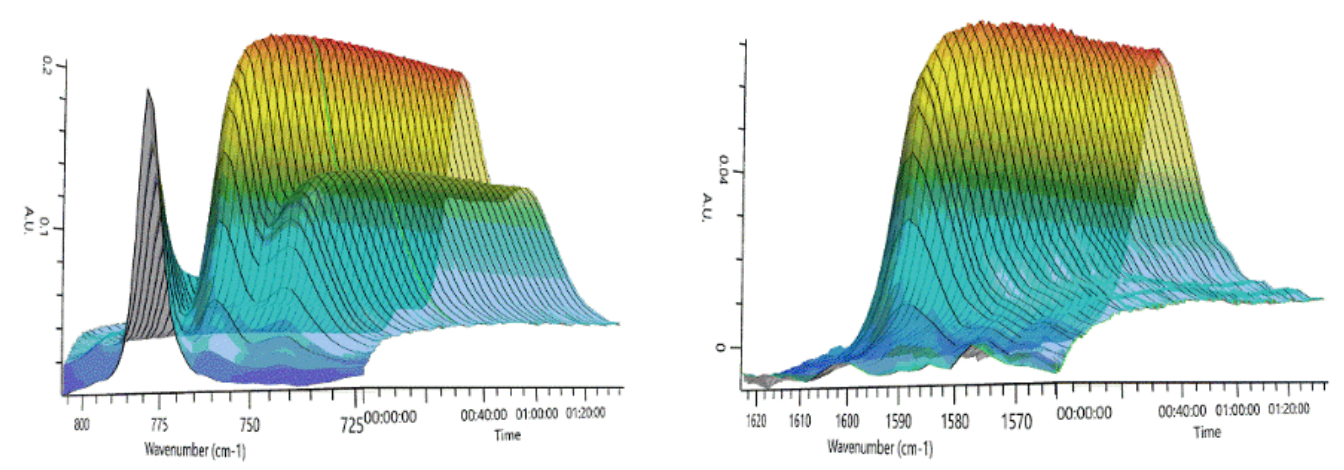

Figure E.2: Frequency analysis of the absorbances of the starting material and the product from Figure 2. Absorbance vs wavenumber. Mettler Toledo iclR 7.0 software. 

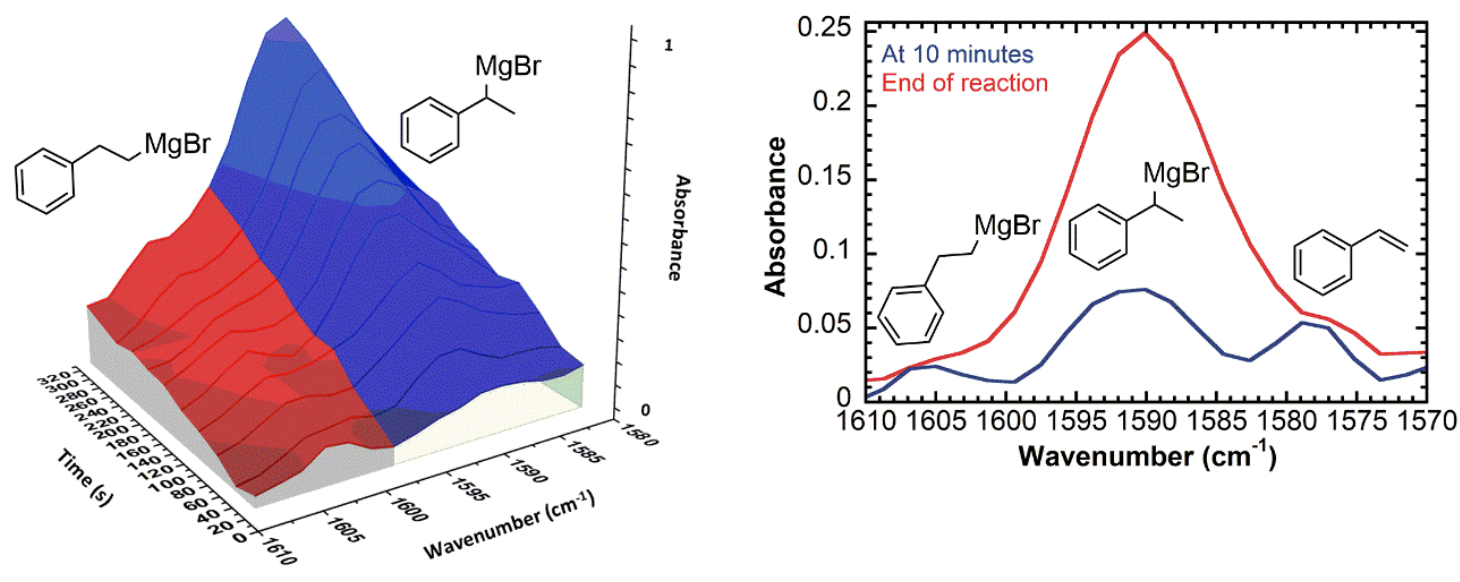

Figure E.3: 3D and 2D interpretation of the overlap of starting materials and products.

\section{Experimental Procedures}

1. Preparation of PDI

Bis(imino)pyridine ligand was synthesized according to literature ${ }^{61,213}$.

2. Preparation of (PDI) $\mathrm{FeCl}_{2}$ catalyst

Bis(imino)pyridine ligand was added to anhydrous ferrous chloride dissolved in THF in a 1:1 fashion. The mixture stirred overnight at room temperature; diethyl ether was added to the resulting in a blue solution. The slurry was filtered to resulting in a blue powder. The powder was dissolved in methylene chloride and filtered to remove unreacted iron (II) chloride. The solution was concentrated in vacuo resulting in a metallic blue or purple powder. Resulting paramagnetic NMR is as stated in literature ${ }^{61,213}$.

3. Preparation of Grignards

All Grignard reagents were synthesized using literature preparations.

According to the following procedure:

In an oven dried 2-neck flask, magnesium (1.2 eq) and iodine (1 crystal) was added and the flask was purged with argon and equipped with a condenser. Diethyl ether (dry) was added and the mixture was brought to reflux. The corresponding alkyl bromide was added dropwise over 1 hour, then the solution stirred for 4 hours. Upon cooling to room temperature, the supernatant was transferred to a second flask and titrated to determine concentration (goal $2 \mathrm{M}$ ). These Grignard reagents were stored at $2-8^{\circ} \mathrm{C}$ in an amber vial under argon. With titration prior to each use. ${ }^{153,188,193,214-216}$ 
4. Typical Reaction time course via ReactIR

Hydromagnesiation reactions were carried out under argon and according to Thomas..$^{153,170,195}$ An oven dried $\left(120^{\circ} \mathrm{C}\right)$ 2-neck reaction flask $(25 \mathrm{~mL})$ was charged with (PDI)FeCl 2 dissolved in $3.0 \mathrm{~mL}$ tetrahydrofuran (THF), then sealed with septa and removed from the glovebox. The flask was purged for 2 minutes with argon then with continued sparging and within 5 $s$ after removing the septa of the center arm the DiComp ReactIR ${ }^{\mathrm{TM}}$ probe under inert atmosphere. Styrene (1 eq standard) was added and the time course was initiated. At 30 seconds, cyclopentyl magnesium bromide (1.5 eq standard) was added by gas tight syringe. IR spectral monitoring continued for 1-8 hours dependent upon catalyst loading. The reaction was then quenched with $\mathrm{CO}_{2}$ for 1 hour, then with $1.0 \mathrm{M}$ solution of hydrochloric acid $(6 \mathrm{~mL})$ overnight. The reactions were extracted 3 times with diethyl ether, washed with brine, dried over sodium sulfate, then concentrated in vacuo. NMR yields were calculated using $16 \mathrm{~mol} \% 1,3,5-$ trimethoxybenzene as an internal standard in $\mathrm{CD}_{3} \mathrm{Cl}$. Example yield calculation and NMR of product are shown in Figure E.4. 


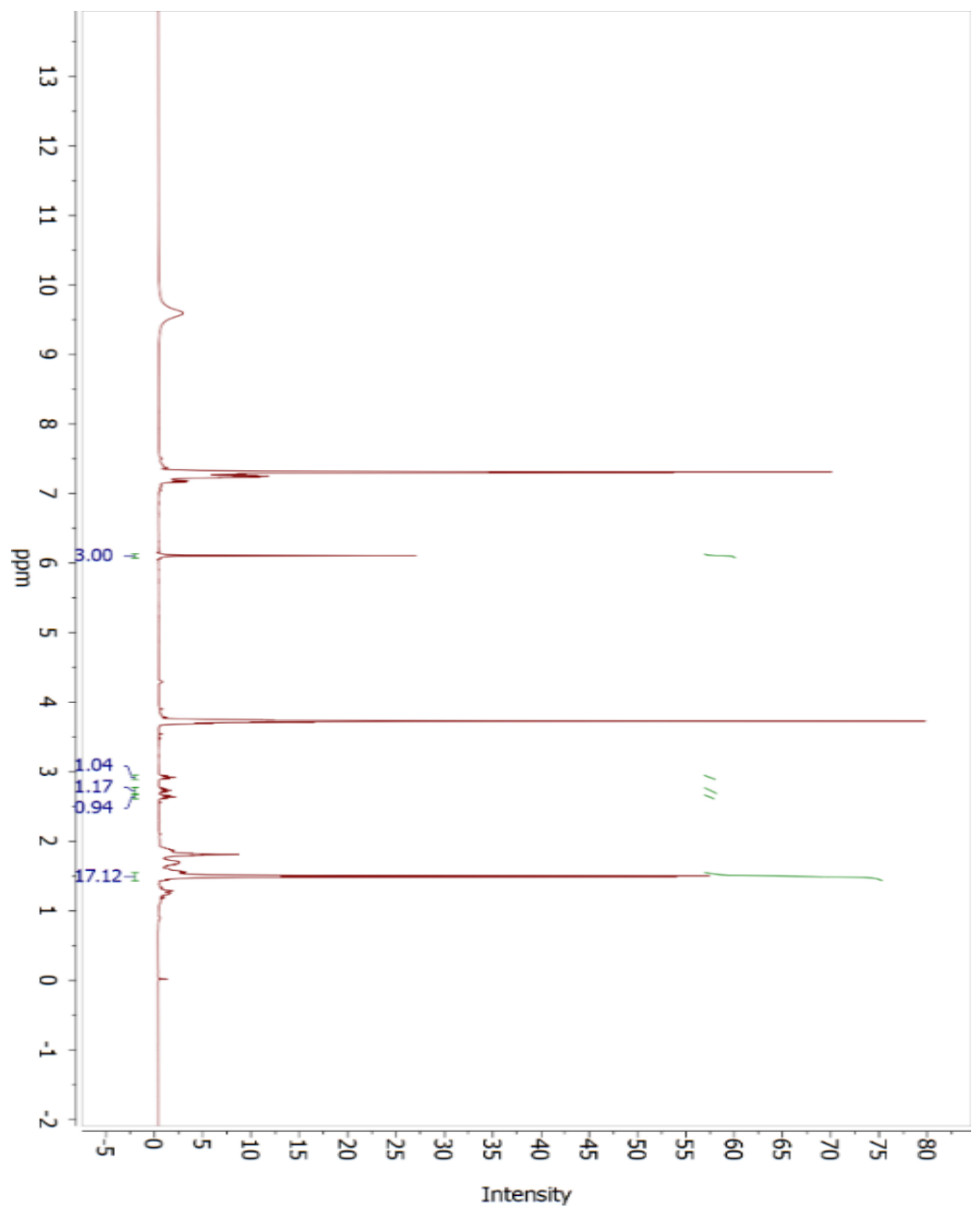

Figure E.4: Typical ${ }^{1} \mathrm{H}$ NMR for hydromagnesiation. Conditions: [styrene] $=0.29 \mathrm{M}$, [cyclopentylMgBr] $=0.43 \mathrm{M},[\mathrm{FePDI}]=0.0029 \mathrm{M}, \mathrm{THF}, 25^{\circ} \mathrm{C}, 2$ hours, quench $\mathrm{CO}_{2} 1$ hour, acidify $1 \mathrm{M} \mathrm{HCl}$, extract diethyl ether $3 \mathrm{x}$, wash brine, dry over sodium sulfate.. 2-phenylpropionic acid: $1.53(\mathrm{~d}, 3 \mathrm{H}) ;(17.12 / 3)^{\star} 16=91 \%$; 3-phenylpropionic acid: 2.96 (t, 2H); (1.04/2)*16 = 8\%; cyclopentanecarboxylic acid: 2.8 (pent, $1 \mathrm{H}$ ); $(1.17 / 1)^{*} 16=19 \%$ 


\section{IGOR Calculations}

The spectra signal for the linear and branched isomers were deconvoluted using a summation of Gaussian curves and global fitting in IGOR, Figure E.5. The summation of Gaussians is described by equation E.1, where the area of each Gaussian shown in Figure E. 3 is calculated. Once the overall area of each $\bar{v}$ was calculated, the intersect of the Gaussians were calculated and used in the

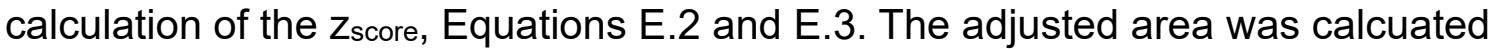
according to equations E.4 and E.5. The initial rates and kobs were calculated from the change in area of each regioisomer using Microsoft Excel non-linear least fit and solver. A typical deconvoluted time course is shown in Figure E.5.

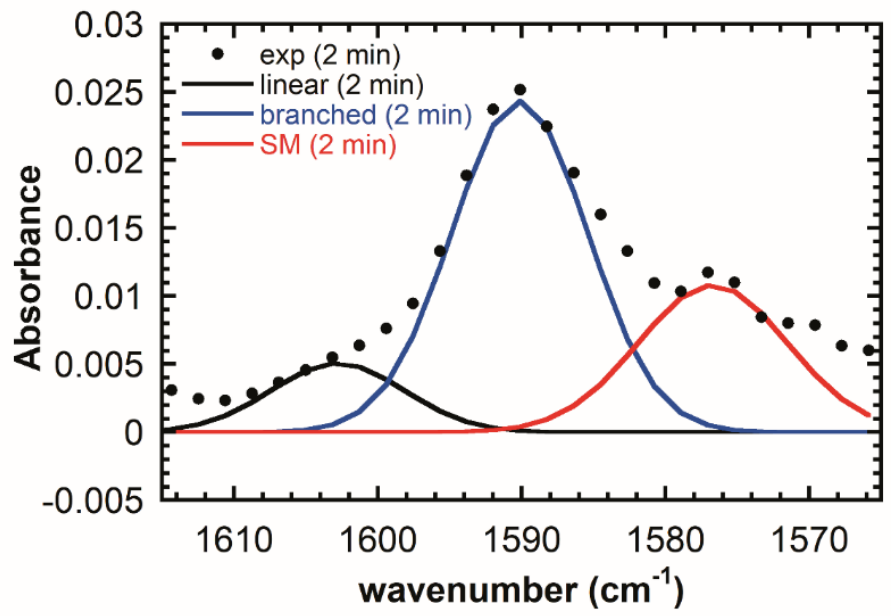

Figure E.5: Frequency analysis of the absorbances of the starting material and the product from Figure 2. Absorbance vs wavenumber.

$$
\begin{aligned}
& \text { Absorbance }=\sum_{t}^{\bar{v}}\left(A b s_{1} * e^{\frac{\left(\frac{x-\bar{v}_{\max }}{\sigma_{1}}\right)^{2}}{2}}+A b s_{2} * e^{\frac{\left(\frac{x-\bar{v}_{\max 2}}{\sigma_{2}}\right)^{2}}{2}}+A b s_{3} * e^{\frac{\left(\frac{x-\bar{v}_{\max }}{\sigma_{3}}\right)^{2}}{2}}\right) \\
& \text { Intersect }_{1}=\frac{\frac{\bar{v}_{\operatorname{max1}}}{\sigma_{1}}+\frac{\bar{y}_{\max 2}}{\sigma_{2}}}{\frac{1}{\sigma_{1}}+\frac{1}{\sigma_{2}}} \\
& \text { Intersect }_{2}=\frac{\frac{\bar{v}_{\max }}{\sigma_{2}}+\frac{\bar{\nu} \max 3}{\sigma_{3}}}{\frac{1}{\sigma_{2}}+\frac{1}{\sigma_{3}}} \\
& \text { zscore }_{1}=\frac{\text { intersect }_{1}-\bar{v}_{\max 1}}{\sigma_{1}} \\
& \text { zscore }_{2}=\frac{\text { intersect }_{2}-\bar{v}_{\max 2}}{\sigma_{2}}
\end{aligned}
$$




$$
\begin{aligned}
& \text { Area }_{1}=\text { Tot. } \text { Area }_{1}-\left(\text { Prob }_{2} * \text { Tot. } \text { Area }_{2}\right)
\end{aligned}
$$

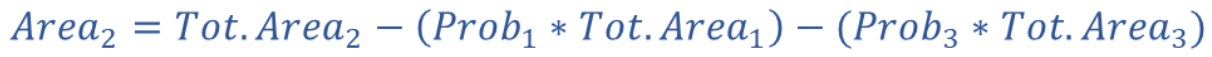

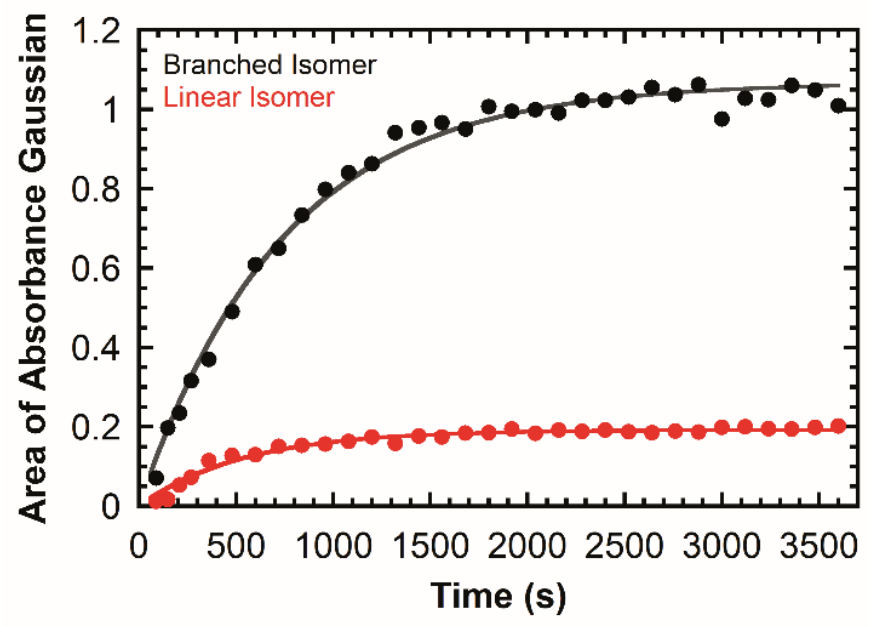

Figure E.6: Typical kinetic time course for hydromagnesiation. Conditions: [styrene $]=0.29 \mathrm{M}$, [cyclopentylMgBr] $=0.43 \mathrm{M},[\mathrm{FePDI}]=0.0029 \mathrm{M}, \mathrm{THF}, 25^{\circ} \mathrm{C}$, 2 hours. 
5. Experimental Dependencies

1. Cyclopentene Dependence

\begin{tabular}{|c|c|c|c|}
\hline $\begin{array}{l}2 \\
1.3 \mathrm{mmol}\end{array}$ & $\frac{\mathrm{l}_{2}(1 \mathrm{~mol} \%)_{)}}{1 \mathrm{~h}, \mathrm{rt}}$ & $\begin{array}{c}4 \\
4 \\
x \mathrm{mmol}\end{array}$ & $\begin{array}{l}\mathrm{Ar}{ }_{\mathrm{Ar}}^{-\mathrm{N}}=2,6-\mathrm{G} \mathrm{Pr}_{2} \mathrm{C}_{6} \mathrm{H}_{3} \\
\mathrm{~N}_{3}\end{array}$ \\
\hline [cyclopentene] (M) & $\begin{array}{l}\text { Initial Rate } \\
\left(\times 10^{-3} \mathrm{~s}^{-1}\right)\end{array}$ & $\begin{array}{c}\text { Kobs } \\
\left(\times 10^{-3} \mathrm{M}^{-1} \mathrm{~s}^{-1}\right)\end{array}$ & $\begin{array}{c}\text { Percent } \\
\text { Yield (\%, B:L) }\end{array}$ \\
\hline $0.0003(0.1 \mathrm{~mol} \%)$ & $1.316 \pm 0.079$ & $1.345 \pm 0.024$ & $97(>10: 1)$ \\
\hline 0.0029 (1mol\%) & $1.801 \pm 0.067$ & $2.177 \pm 0.041$ & $94(10: 1)$ \\
\hline 0.0058 (2mol\%) & $1.654 \pm 0.067$ & $1.337 \pm 0.028$ & $98(>10: 1)$ \\
\hline $0.0146(5 \mathrm{~mol} \%)$ & $1.695 \pm 0.083$ & $1.922 \pm 0.069$ & $77(10: 1)$ \\
\hline 0.291 (100mol\%) & $1.714 \pm 0.075$ & $1.811 \pm 0.070$ & $79(>10: 1)$ \\
\hline $1.456(500 \mathrm{~mol} \%)$ & $1.804 \pm 0.101$ & $2.166 \pm 0.051$ & $58(7: 1)$ \\
\hline 2.912 (1000mol\%) & $1.883 \pm 0.211$ & $2.224 \pm 0.104$ & $55(>10: 1)$ \\
\hline 4.369 (1500mol\%) & $1.816 \pm 0.185$ & $3.158 \pm 0.231$ & $50(>10: 1)$ \\
\hline $5.825(2000 \mathrm{~mol} \%)$ & $1.025 \pm 0.179$ & $1.062 \pm 0.054$ & $49(>10: 1)$ \\
\hline
\end{tabular}

Table E.1: Initial rate kinetics and calculated kobs for branched isomer. Conditions: 0.291 M 1; 2.91 mM (PDI IPr)FeCl2; 0.434 M 2; x M 4; 1 hour; THF; rt. 

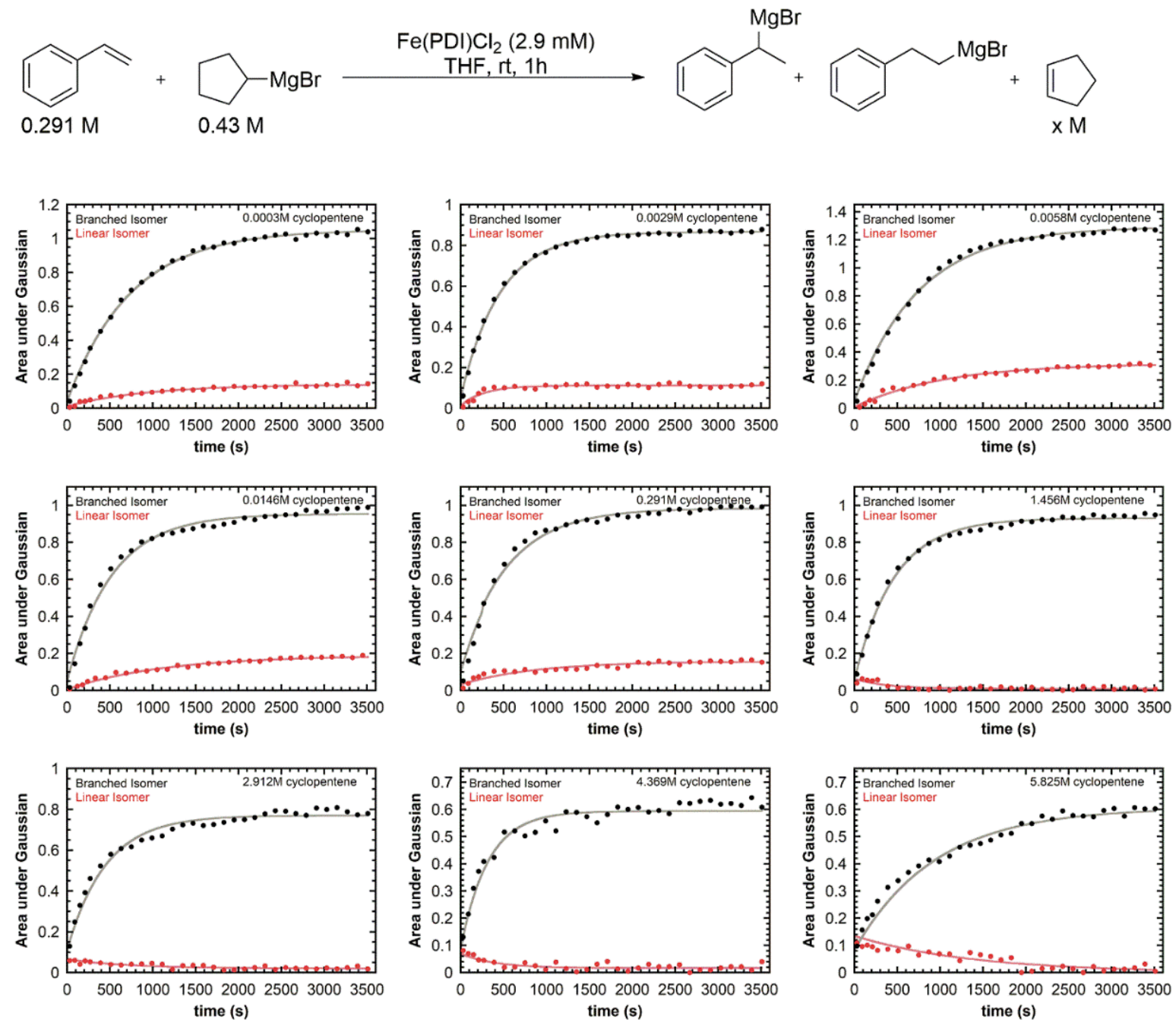

Figure E.8: Reaction time courses from Table S.1, [(1-phenylethyl)MgBr] vs time. Conditions: $0.291 \mathrm{M} \mathrm{1;2.91} \mathrm{mM} \mathrm{(PDIPr)} \mathrm{FeCl}_{2} ; 0.434 \mathrm{M}$ 2; x mol\% 4; 1 hour; THF; rt 
2. Catalyst Dependence

\begin{tabular}{cccc}
\hline $\begin{array}{c}{\left[\left(\mathrm{PDI}^{\mathrm{Pr}}\right) \mathrm{FeCl}_{2}\right]} \\
(\mathrm{mM})\end{array}$ & $\begin{array}{c}\text { Initial Rate } \\
\left(\times 10^{-3} \mathrm{~s}^{-1}\right)\end{array}$ & $\begin{array}{c}\text { Kobs } \\
\left(\times 10^{-3} \mathrm{M}^{-1} \mathrm{~s}^{-1}\right)\end{array}$ & $\begin{array}{c}\text { Percent } \\
\text { Yield }(\%, \mathrm{~B}: \mathrm{L})\end{array}$ \\
\hline $0.55(0.2 \mathrm{~mol} \%)$ & $0.395 \pm 0.061$ & $0.272 \pm 0.043$ & $98(>10: 1)$ \\
$1.09(0.4 \mathrm{~mol} \%)$ & $0.521 \pm 0.052$ & $0.578 \pm 0.043$ & $98(10: 1)$ \\
$1.64(0.5 \mathrm{~mol} \%)$ & $0.877 \pm 0.059$ & $0.864 \pm 0.016$ & $96(>10: 1)$ \\
$2.19(0.8 \mathrm{~mol} \%)$ & $0.965 \pm 0.036$ & $1.369 \pm 0.036$ & $94(10: 1)$ \\
$2.91(1.0 \mathrm{~mol} \%)$ & $1.099 \pm 0.065$ & $1.029 \pm 0.016$ & $96(10: 1)$ \\
$4.38(1.5 \mathrm{~mol} \%)$ & $1.739 \pm 0.064$ & $2.224 \pm 0.033$ & $97(7: 1)$ \\
$6.02(2.1 \mathrm{~mol} \%)$ & $1.959 \pm 0.153$ & $3.423 \pm 0.103$ & $81(>10: 1)$ \\
$7.67(2.6 \mathrm{~mol} \%)$ & $2.116 \pm 0.273$ & $4.999 \pm 0.267$ & $95(>10: 1)$ \\
$8.76(3.0 \mathrm{~mol} \%)$ & $2.258 \pm 0.216$ & $5.104 \pm 0.215$ & $89(>10: 1)$
\end{tabular}

Table E.2: Initial rate kinetics and calculated kobs for branched isomer Conditions: $0.291 \mathrm{M} 1 ; x \mathrm{mM}\left(\mathrm{PDI}{ }^{\mathrm{PPr}}\right) \mathrm{FeCl}_{2} ; 0.434 \mathrm{M}$ 2; 1 hour; THF; rt. Standard reaction in red 

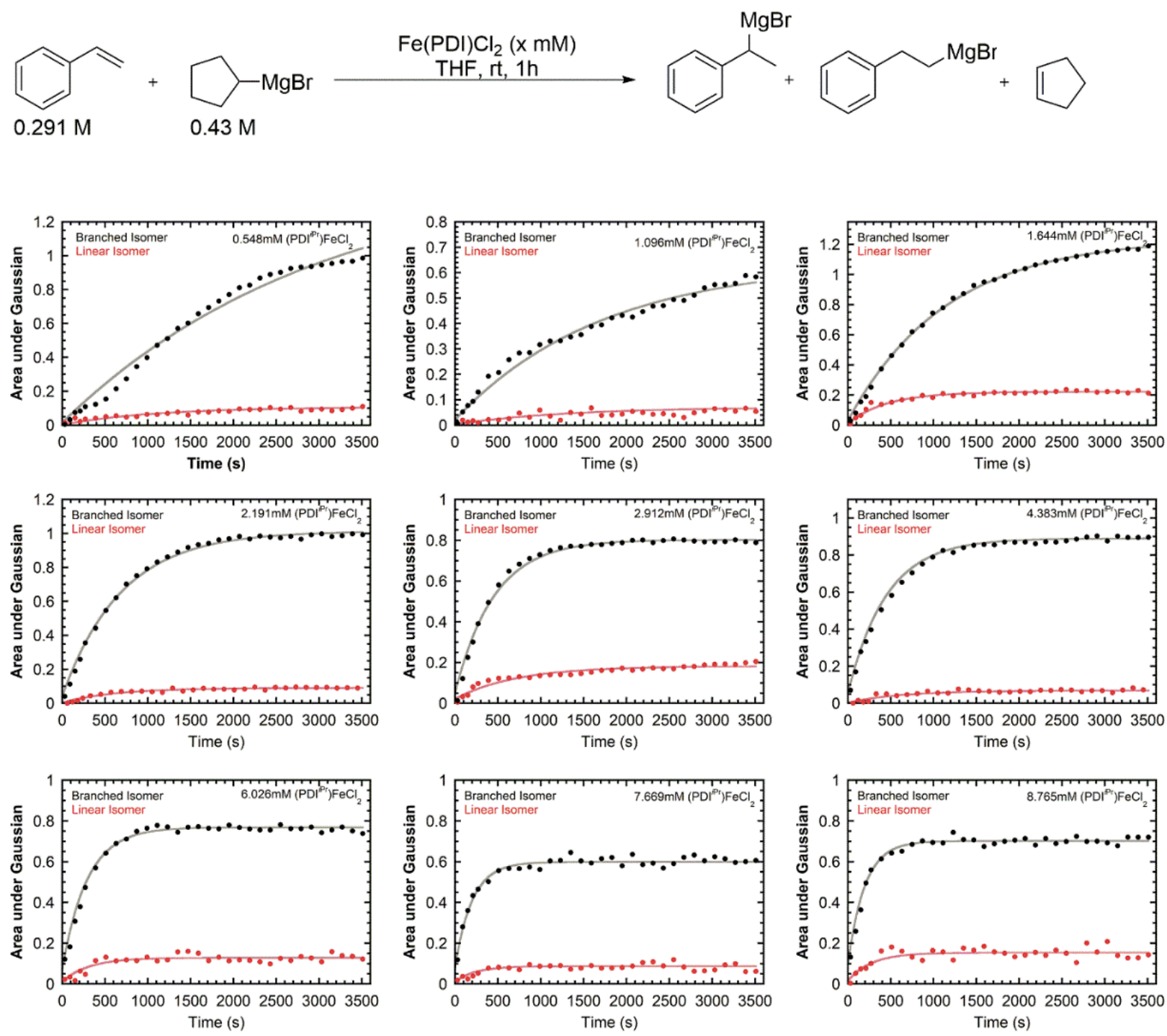

Figure E.9: Reaction time courses from Table S.2, [(1-phenylethyl)MgBr] vs time. Conditions: $0.291 \mathrm{M} \mathrm{1;} \mathrm{x} \mathrm{mM} \mathrm{(PDIPr)FeCl} 2 ; 0.434$ M 2; 1 hour; THF; rt 


\section{Grignard Dependence}

\begin{tabular}{cccc}
\hline $\begin{array}{c}\text { CyclopentylMgBr] } \\
(\mathrm{M})\end{array}$ & $\begin{array}{c}\text { Initial Rate } \\
\left(\times 10^{-3} \mathrm{~s}^{-1}\right)\end{array}$ & $\begin{array}{c}\text { kobs } \\
\left(\times 10^{-3} \mathrm{M}^{-1} \mathrm{~s}^{-1}\right)\end{array}$ & $\begin{array}{c}\text { Percent } \\
\text { Yield }(\%, \mathrm{~B}: \mathrm{L})\end{array}$ \\
\hline 0.0804 & $0.439 \pm 0.063$ & $4.392 \pm 0.529$ & $81(>10: 1)$ \\
0.141 & $0.760 \pm 0.102$ & $2.748 \pm 0.174$ & $98(10: 1)$ \\
0.322 & $1.076 \pm 0.109$ & $1.118 \pm 0.021$ & $65(>10: 1)$ \\
0.429 & $1.099 \pm 0.065$ & $0.983 \pm 0.016$ & $96(10: 1)$ \\
0.858 & $1.532 \pm 0.108$ & $3.670 \pm 0.199$ & $94(6: 1)$ \\
1.715 & $1.975 \pm 0.209$ & $4.341 \pm 0.229$ & $92(7: 1)$
\end{tabular}

Table E.3: Initial rate kinetics and calculated kobs Conditions: $0.291 \mathrm{M} \mathrm{1;2.91}$ $\mathrm{mM}\left(\mathrm{PDI}{ }^{\mathrm{PPr}}\right) \mathrm{FeCl}_{2}$; $\mathrm{x}$ M 2; 1 hour; THF; rt. Standard reaction in red
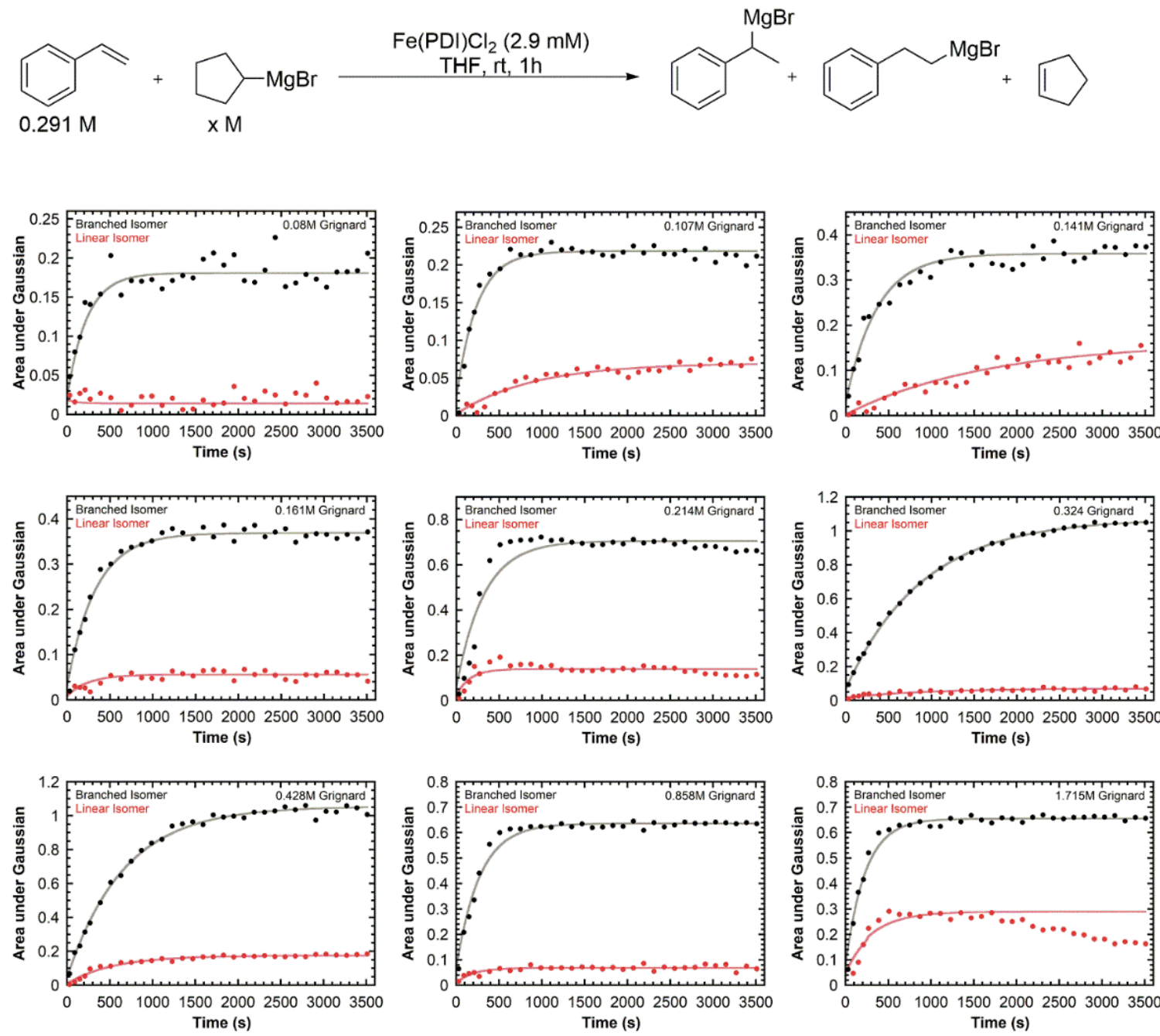

Figure E.10: Reaction time courses from Table S.3, [(1-phenylethyl)MgBr] vs time. Conditions: $\left.0.291 \mathrm{M} \mathrm{1;2.9} \mathrm{mM} \mathrm{(PDI}{ }^{\mathrm{PPr}}\right) \mathrm{FeCl}_{2} ; \mathrm{x} \mathrm{M}$ 2; 1 hour; THF; rt. 
4. Styrene Dependence

\begin{tabular}{cccc}
\hline $\begin{array}{c}\text { Styrene] } \\
(\mathrm{M})\end{array}$ & $\begin{array}{c}\text { Initial Rate } \\
\left(\times 10^{-3} \mathrm{~s}^{-1}\right)\end{array}$ & $\begin{array}{c}\text { kobs } \\
\left(\times 10^{-3} \mathrm{M}^{-1} \mathrm{~s}^{-1}\right)\end{array}$ & $\begin{array}{c}\text { Percent } \\
\text { Yield }(\%, \mathrm{~B}: \mathrm{L})\end{array}$ \\
\hline 0.073 & $0.794 \pm 0.028$ & $3.106 \pm 0.238$ & $96(2: 1)$ \\
0.146 & $0.903 \pm 0.066$ & $2.729 \pm 0.189$ & $>99(6: 1)$ \\
0.218 & $0.919 \pm 0.097$ & $1.841 \pm 0.087$ & $>99(>10: 1)$ \\
0.291 & $1.099 \pm 0.065$ & $0.983 \pm 0.016$ & $96(10: 1)$ \\
0.437 & $1.228 \pm 0.048$ & $0.715 \pm 0.014$ & $67(>10: 1)$ \\
0.582 & $1.517 \pm 0.084$ & $1.016 \pm 0.031$ & $65(7: 1)$ \\
1.16 & $1.363 \pm 0.036$ & $1.131 \pm 0.029$ & $55(>10: 1)$ \\
1.75 & $1.234 \pm 0.018$ & $0.931 \pm 0.023$ & $39(7: 1)$ \\
2.33 & $1.092 \pm 0.027$ & $1.331 \pm 0.027$ & $38(4: 1)$
\end{tabular}

Table E.4: Initial rate kinetics and calculated kobs for the branched isomer Conditions: x M 1; 2.91 mM (PDIPr)FeCl 2 ; 0.434 M 2; 1 hour; THF; rt. Standard reaction in red 

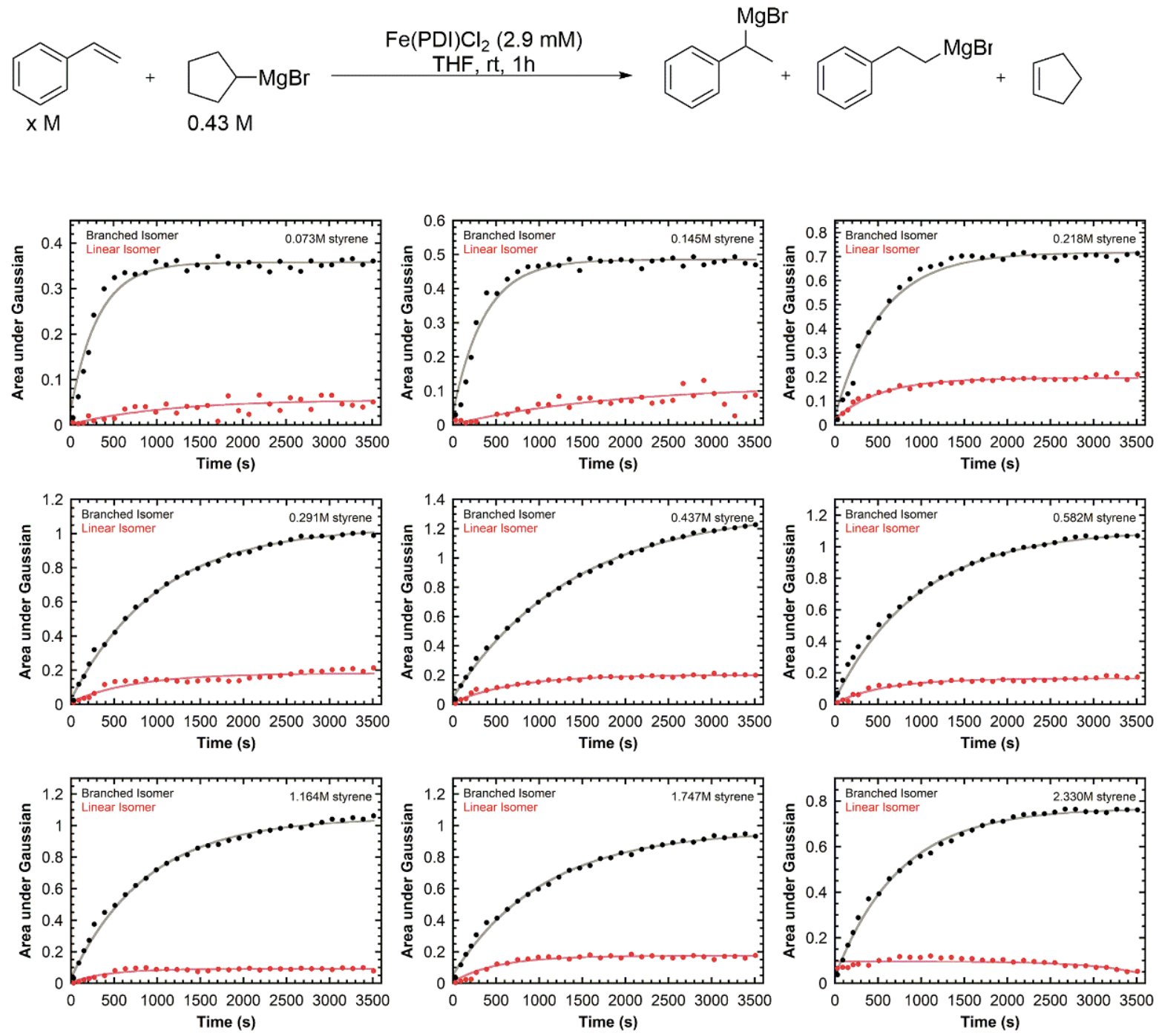

Figure E.11: Reaction time courses from Table S.4, [(1-phenylethyl)MgBr] vs time. Conditions: x M 1; 2.9 mM (PDIPr)FeCl2; 0.434 M 2; 1 hour; THF; rt. 


\begin{tabular}{|c|c|c|c|c|}
\hline & & 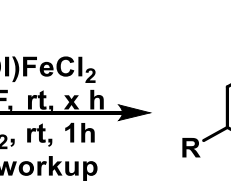 & $\mathrm{CO}_{2} \mathrm{H}$ & \\
\hline $\mathrm{R}=$ tert $\cdot$ buty $\mathrm{l}$ & obutyl, methyl, $n$ & 10xy & & \\
\hline $\begin{array}{l}\text { Styrene } \\
\text { Derivative }\end{array}$ & [styrene] (M) & $\begin{array}{l}\text { Initial Rate } \\
\left(\times 10^{-3} \mathrm{~s}^{-1}\right)\end{array}$ & $\begin{array}{c}\text { Kobs } \\
\left(\times 10^{-3} \mathrm{M}^{-1} \mathrm{~s}^{-1}\right)\end{array}$ & $\begin{array}{c}\text { Percent } \\
\text { Yield }(\%, B: L)\end{array}$ \\
\hline p-methyl & 0.073 & $2.36 \pm 0.20$ & $2.65 \pm 0.29$ & $91(3: 1)$ \\
\hline p-methyl & 0.146 & $3.37 \pm 0.89$ & $1.98 \pm 0.12$ & $89(5: 1)$ \\
\hline p-methyl & 0.291 & $5.64 \pm 0.047$ & $1.96 \pm 0.04$ & $95(4: 1)$ \\
\hline p-methyl & 0.428 & $6.18 \pm 0.24$ & $1.15 \pm 0.01$ & $91(4: 1)$ \\
\hline p-methyl & 0.582 & $6.77 \pm 0.37$ & $0.89 \pm 0.01$ & $93(10: 1)$ \\
\hline p-methyl & 1.16 & $7.50 \pm 0.58$ & $1.05 \pm 0.03$ & $99(>20: 1)$ \\
\hline p-isobutyl & 0.073 & $0.76 \pm 0.40$ & $2.17 \pm 0.56$ & $81(4: 1)$ \\
\hline p-isobutyl & 0.146 & $1.95 \pm 0.33$ & $1.65 \pm 0.09$ & $96(3: 1)$ \\
\hline p-isobutyl & 0.238 & $3.03 \pm 0.55$ & $1.50 \pm 0.09$ & $85(4: 1)$ \\
\hline p-isobutyl & 0.291 & $3.19 \pm 0.12$ & $1.42 \pm 0.04$ & $86(5: 1)$ \\
\hline p-isobutyl & 0.428 & $4.86 \pm 0.79$ & $1.40 \pm 0.05$ & $90(7: 1)$ \\
\hline p-isobutyl & 0.582 & $5.00 \pm 0.57$ & $0.94 \pm 0.02$ & $96(4: 1)$ \\
\hline p-tert-butyl & 0.073 & $2.65 \pm 0.90$ & $3.18 \pm 1.22$ & $98(3: 1)$ \\
\hline p-tert-butyl & 0.146 & $4.24 \pm 0.86$ & $4.18 \pm 0.60$ & $94(3: 1)$ \\
\hline p-tert-butyl & 0.291 & $8.79 \pm 0.67$ & $2.98 \pm 0.19$ & $98(3: 1)$ \\
\hline p-tert-butyl & 0.428 & $10.05 \pm 1.62$ & $2.55 \pm 0.09$ & $92(5: 1)$ \\
\hline p-tert-butyl & 0.582 & $12.75 \pm 1.89$ & $2.58 \pm 0.08$ & $96(22: 1)$ \\
\hline p-tert-butyl & 1.16 & $13.63 \pm 0.86$ & $2.30 \pm 0.02$ & $76(>20: 1)$ \\
\hline p-methoxy & 0.073 & $3.16 \pm 0.58$ & $3.18 \pm 0.36$ & $77(1: 1)$ \\
\hline p-methoxy & 0.146 & $9.09 \pm 0.65$ & $2.83 \pm 0.18$ & $85(1: 1)$ \\
\hline p-methoxy & 0.291 & $13.13 \pm 2.25$ & $2.58 \pm 0.08$ & $70(2: 1)$ \\
\hline p-methoxy & 0.428 & $15.18 \pm 0.42$ & $1.31 \pm 0.04$ & $83(2: 1)$ \\
\hline p-methoxy & 0.582 & $17.34 \pm 1.58$ & $1.27 \pm 0.03$ & $86(3: 1)$ \\
\hline p-methoxy & 1.16 & $18.01 \pm 2.18$ & $1.22 \pm 0.02$ & $87(3: 1)$ \\
\hline
\end{tabular}

Table E.5: Initial rate kinetics and calculated kobs for branched isomer Conditions: x M 1; 2.91 mM Fe(PDIPr) $\mathrm{Cl}_{2} ; 0.434$ M 2; 0 M 4; 1 hour; THF; rt. Standard concentration used for Hammett Correlation in Red. 

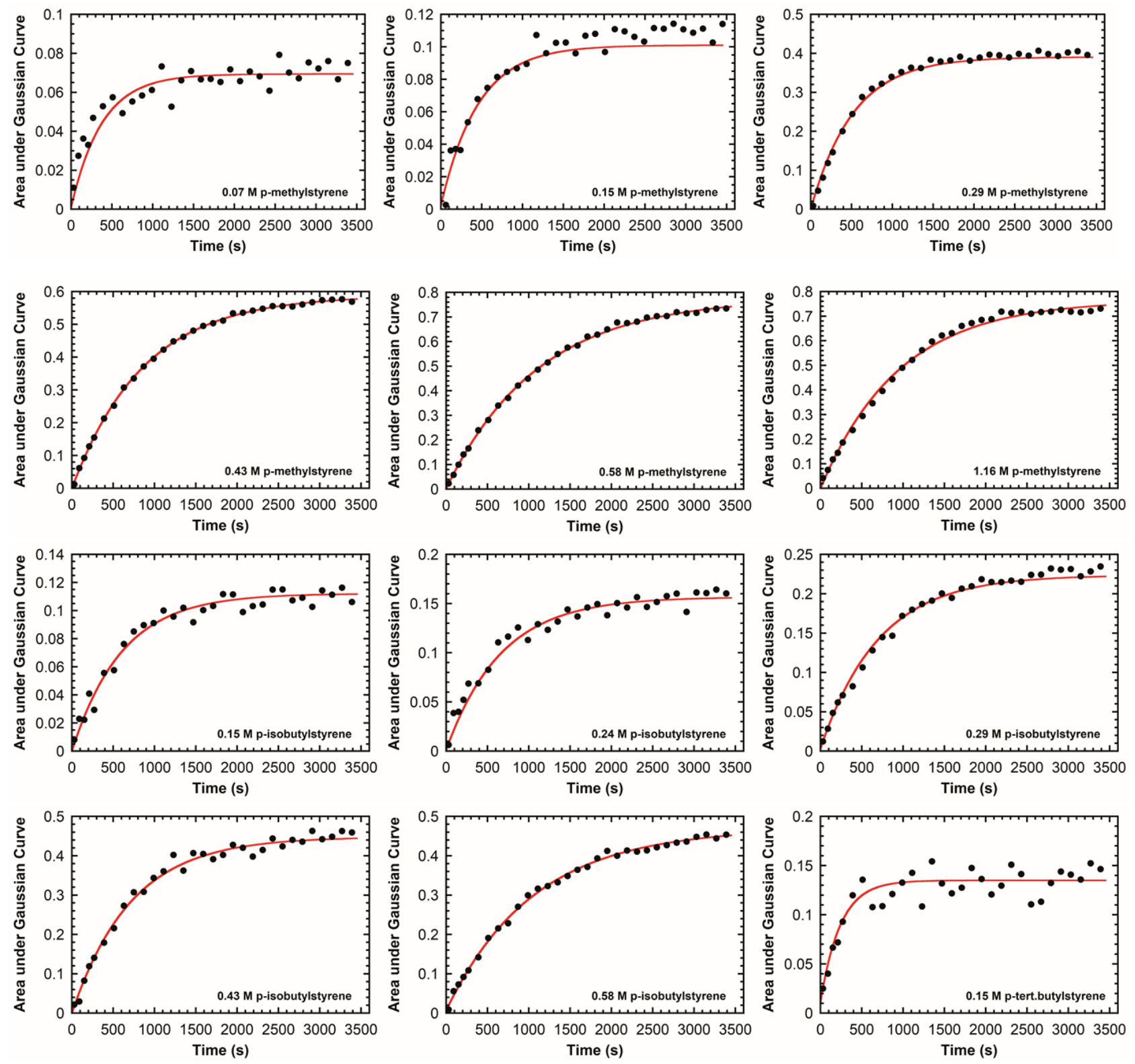

${ }^{*}$ Continued on page E18 

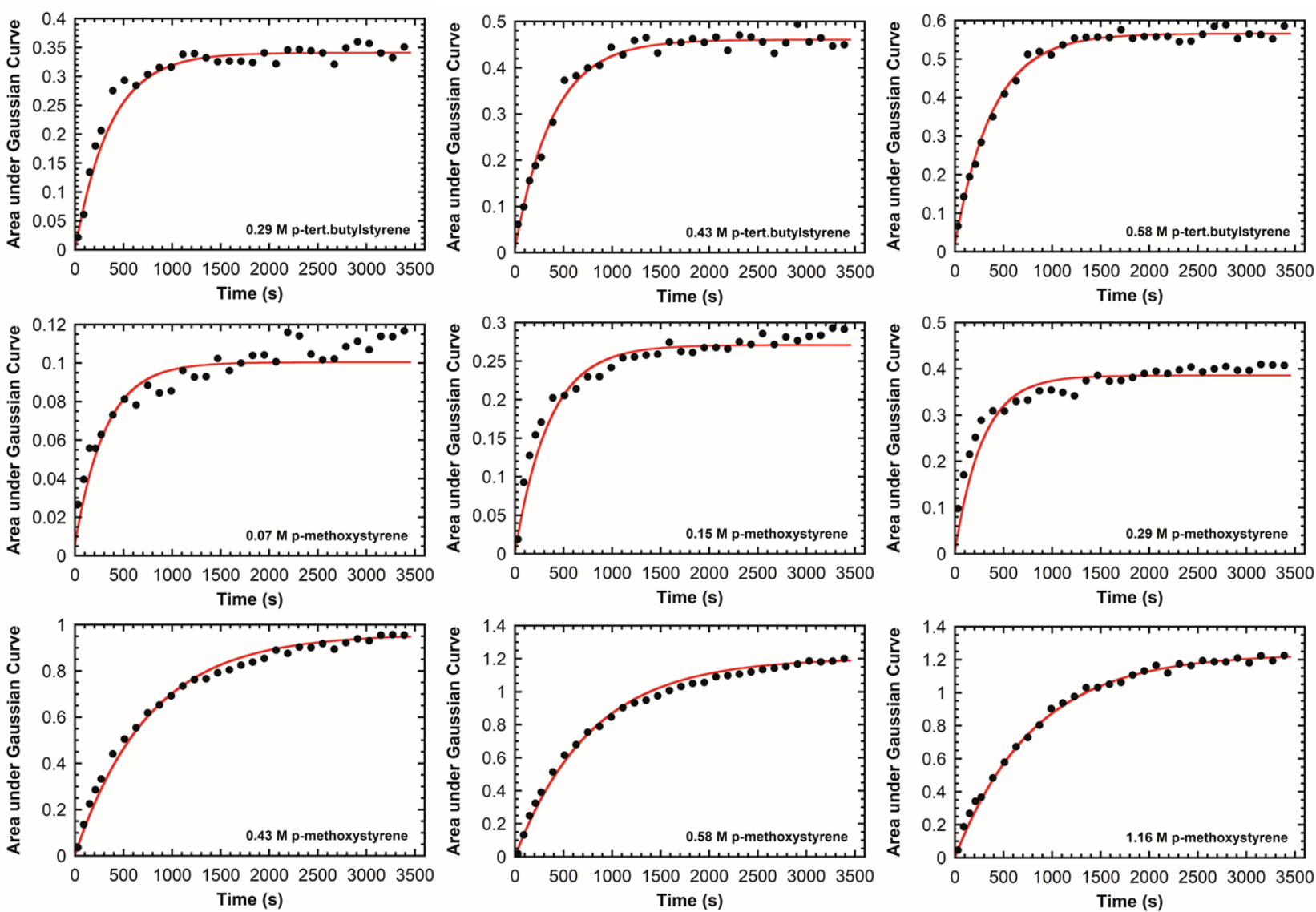

Figure E.12: Reaction time courses from Table E.14, [(1-(4-substituted) phenylethyl) $\mathrm{MgBr}$ ] vs time. Conditions: x M 1; 2.9 mM (PDIPr)FeCl2; $0.434 \mathrm{M} \mathrm{2;} 1$ hour; THF; rt. *See Figure for derivative concentrations. 
6. Grignard Reagents
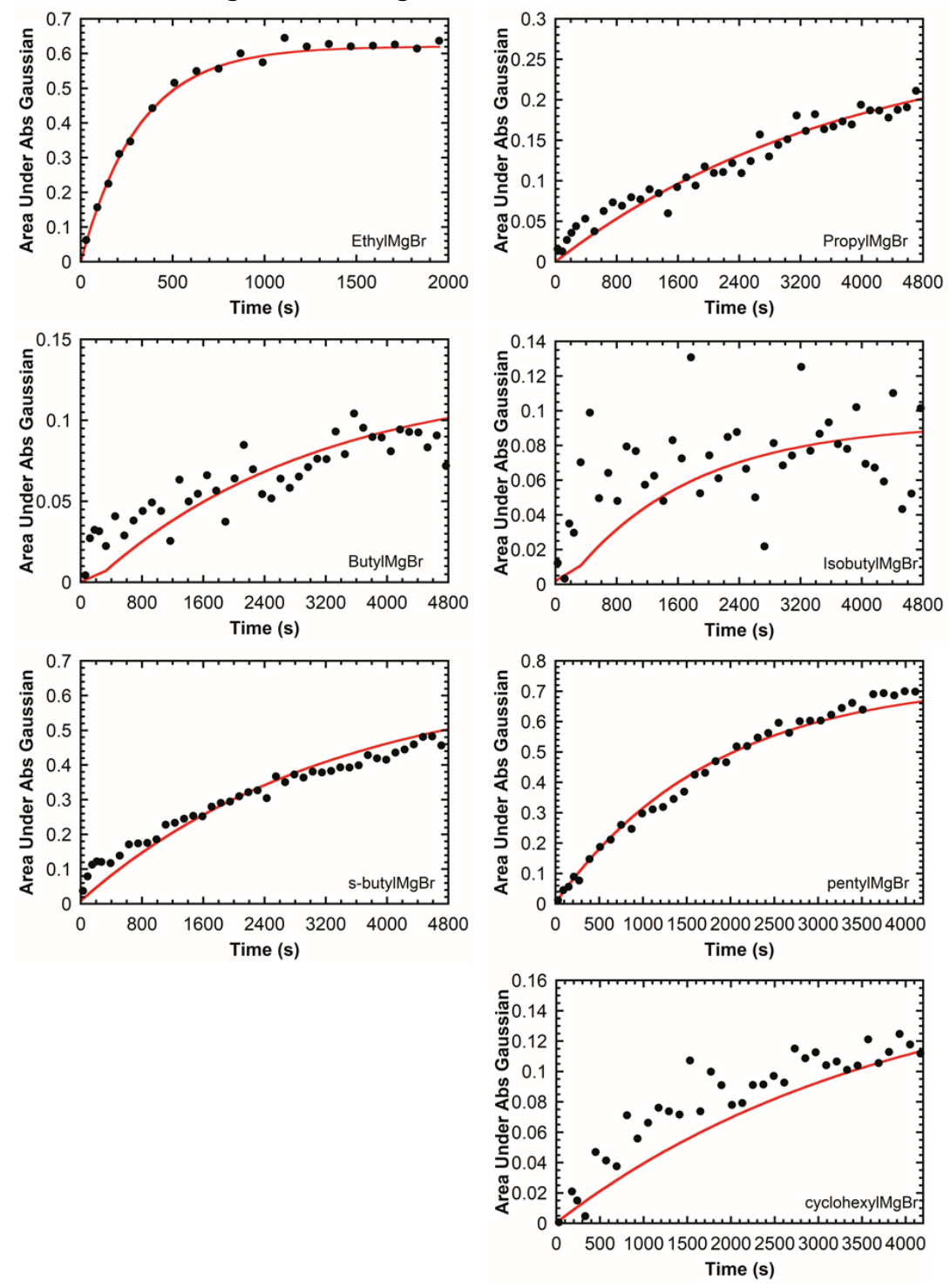
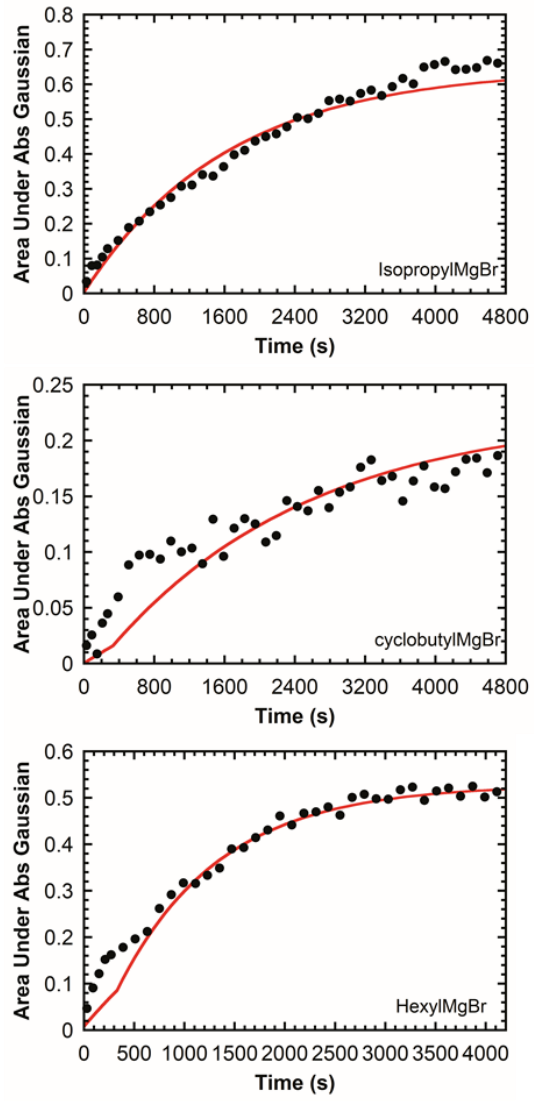

Figure E.13: [(1-phenylethyl) MgBr] vs time. Conditions: $0.29 \mathrm{M} \mathrm{S} ; 2.9 \mathrm{mM}$ (PDIPr)FeCl2; $0.434 \mathrm{M} \mathrm{G}^{\mathrm{x}} ; 1$ hour; THF; rt. *See Figure for derivative concentrations. 
7. EthylMgBr (Stir)
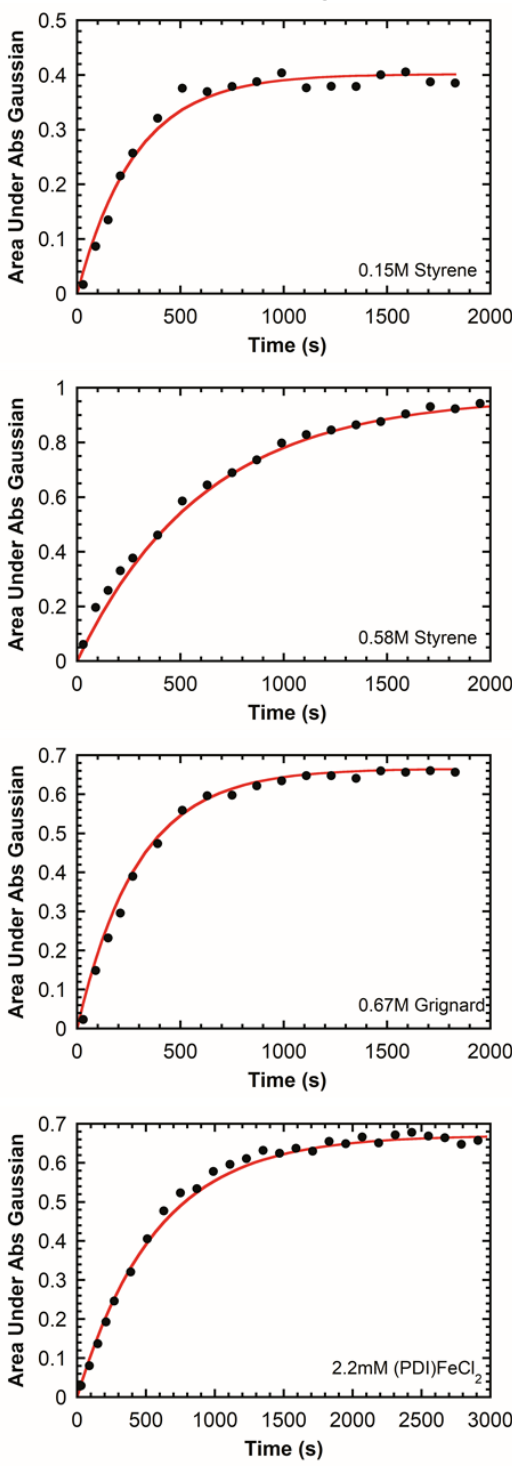
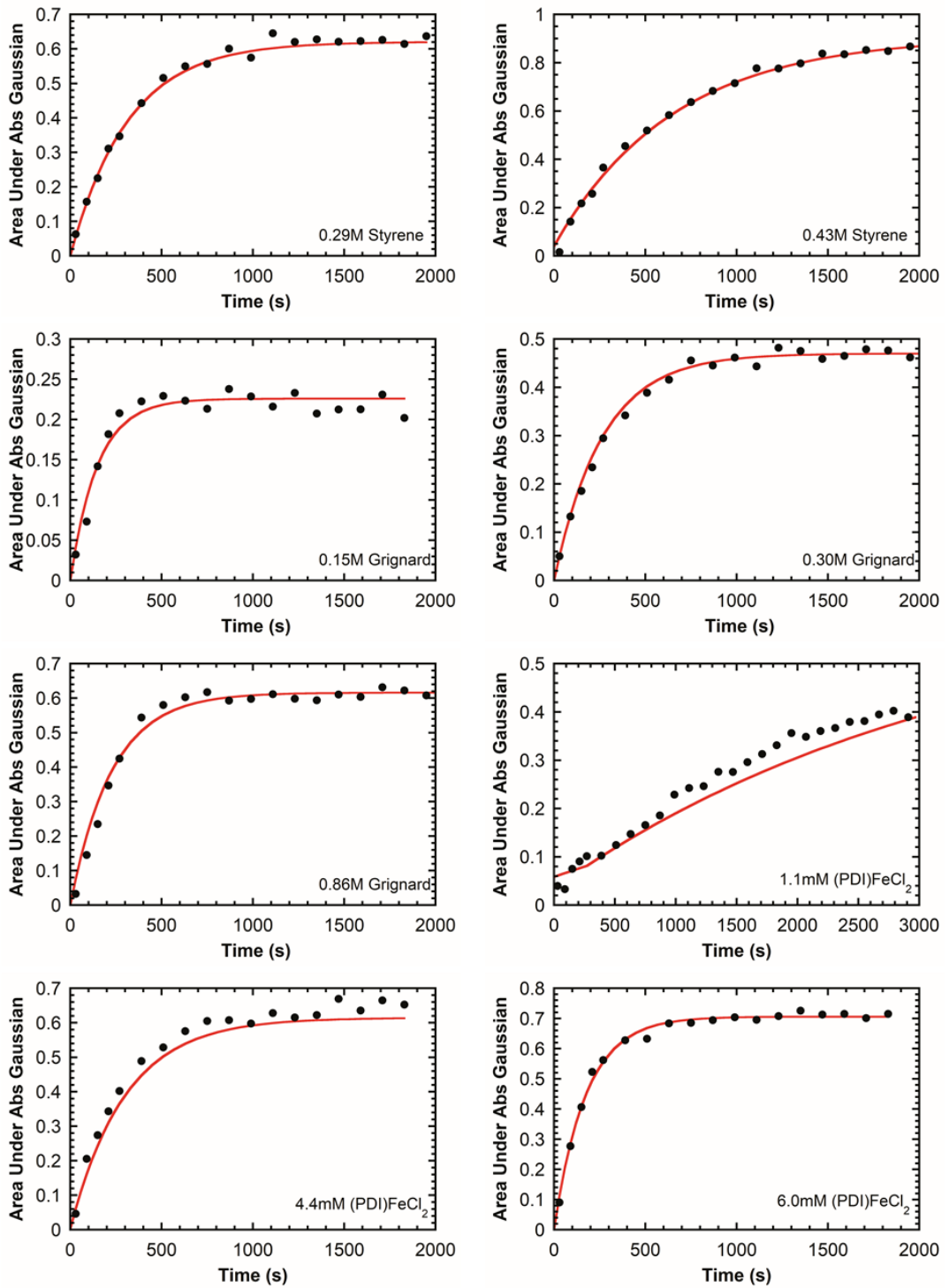

Figure E.14: [(1-phenylethyl)MgBr] vs time. Standard Conditions: 0.29 M S; 2.9 mM $(\mathrm{PDIPr}) \mathrm{FeCl}_{2} ; 0.434 \mathrm{M} \mathrm{GEt}^{\mathrm{Et}} 1$ hour; THF; rt. *See Figure for varied concentrations. 

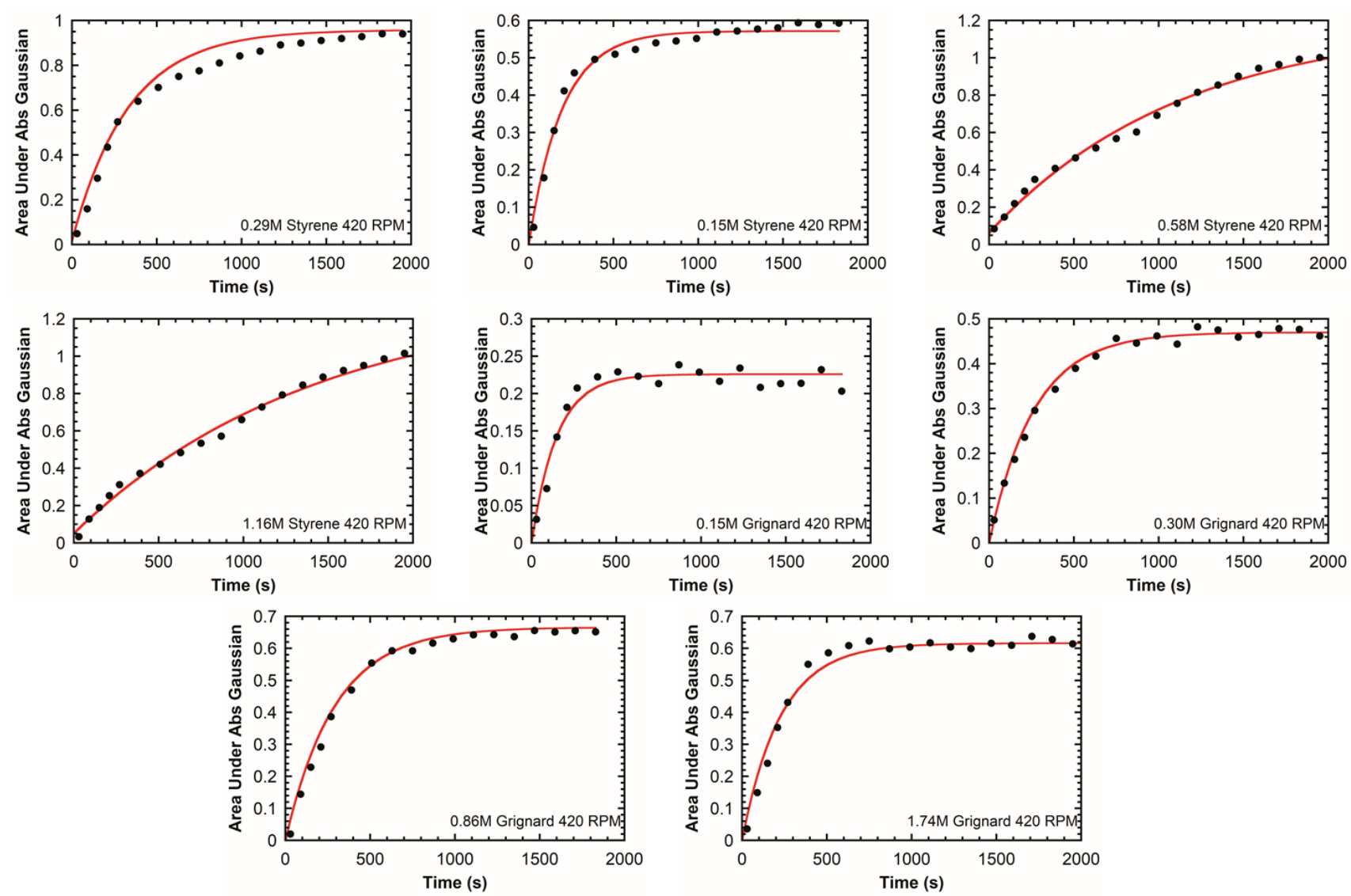

Figure E.15: [(1-phenylethyl)MgBr] vs time. Standard Conditions: $0.29 \mathrm{M} \mathrm{S} ; 2.9 \mathrm{mM}$ (PDIPr)FeCl $2 ; 0.434 \mathrm{M} \mathrm{G}^{\mathrm{Et}} ; 1$ hour; THF; rt, 420 RPM. *See Figure for derivative concentrations. 
8. IsopropylMgBr
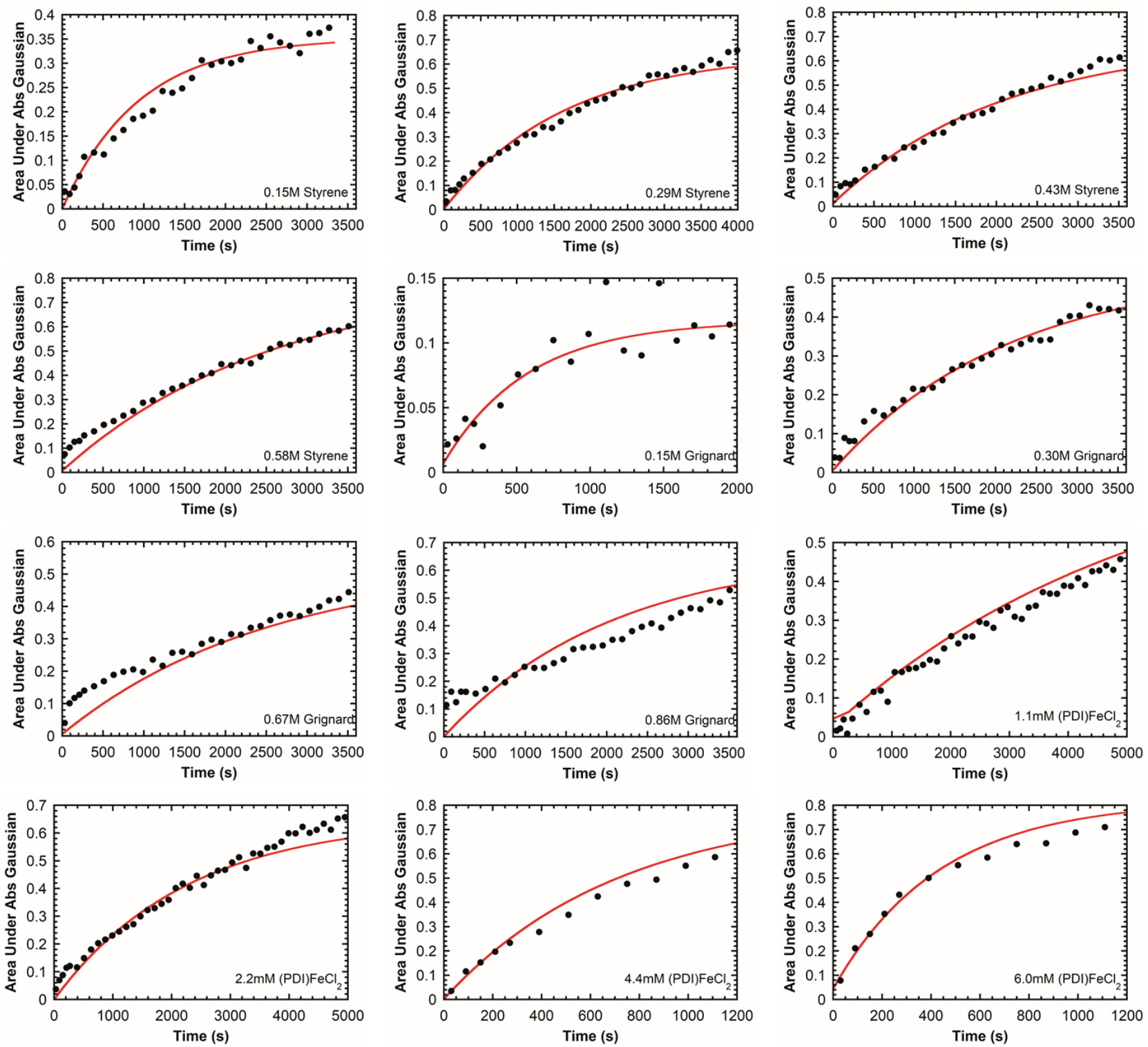

Figure E.16: [(1-phenylethyl)MgBr] vs time. Standard Conditions: $0.29 \mathrm{M} \mathrm{S} ; 2.9 \mathrm{mM}$ $\left(\mathrm{PD}{ }^{\mathrm{iPr}}\right) \mathrm{FeCl}_{2} ; 0.434 \mathrm{M}$ GiPr; 1 hour; THF; rt. *See Figure for derivative concentrations. 


\section{PentylMgBr}
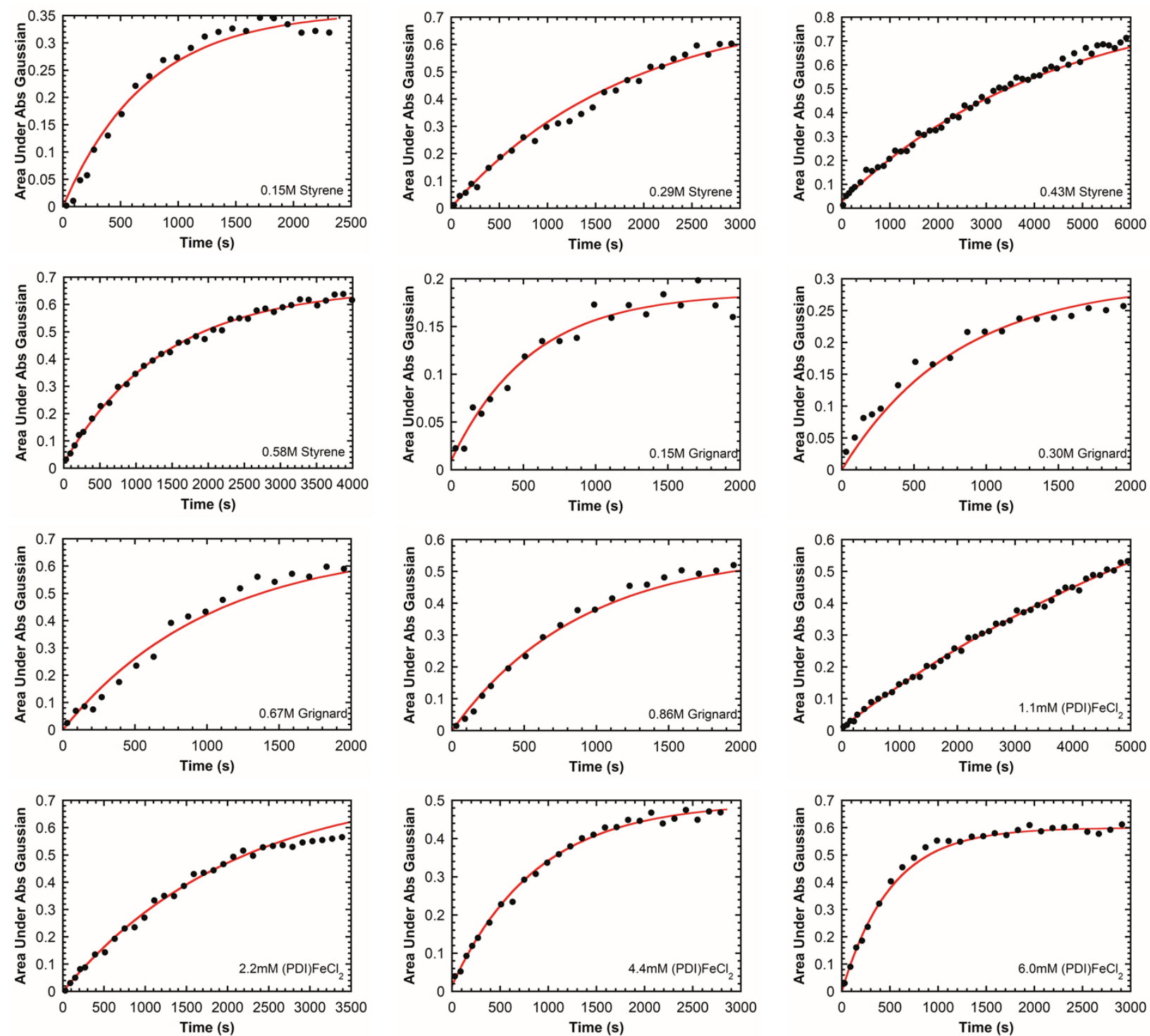

Figure E.17: [(1-phenylethyl)MgBr] vs time. Standard Conditions: $0.29 \mathrm{M} \mathrm{S} ; 2.9 \mathrm{mM}$ $(\mathrm{PDIPr}) \mathrm{FeCl}_{2} ; 0.434 \mathrm{M}$ GPent; 1 hour; THF; rt. *See Figure for derivative concentrations. 


\section{Precatalysts}

\section{a. $\left(\left.\mathrm{PDI}\right|^{\mathrm{Me}}\right) \mathrm{FeCl}_{2}$}
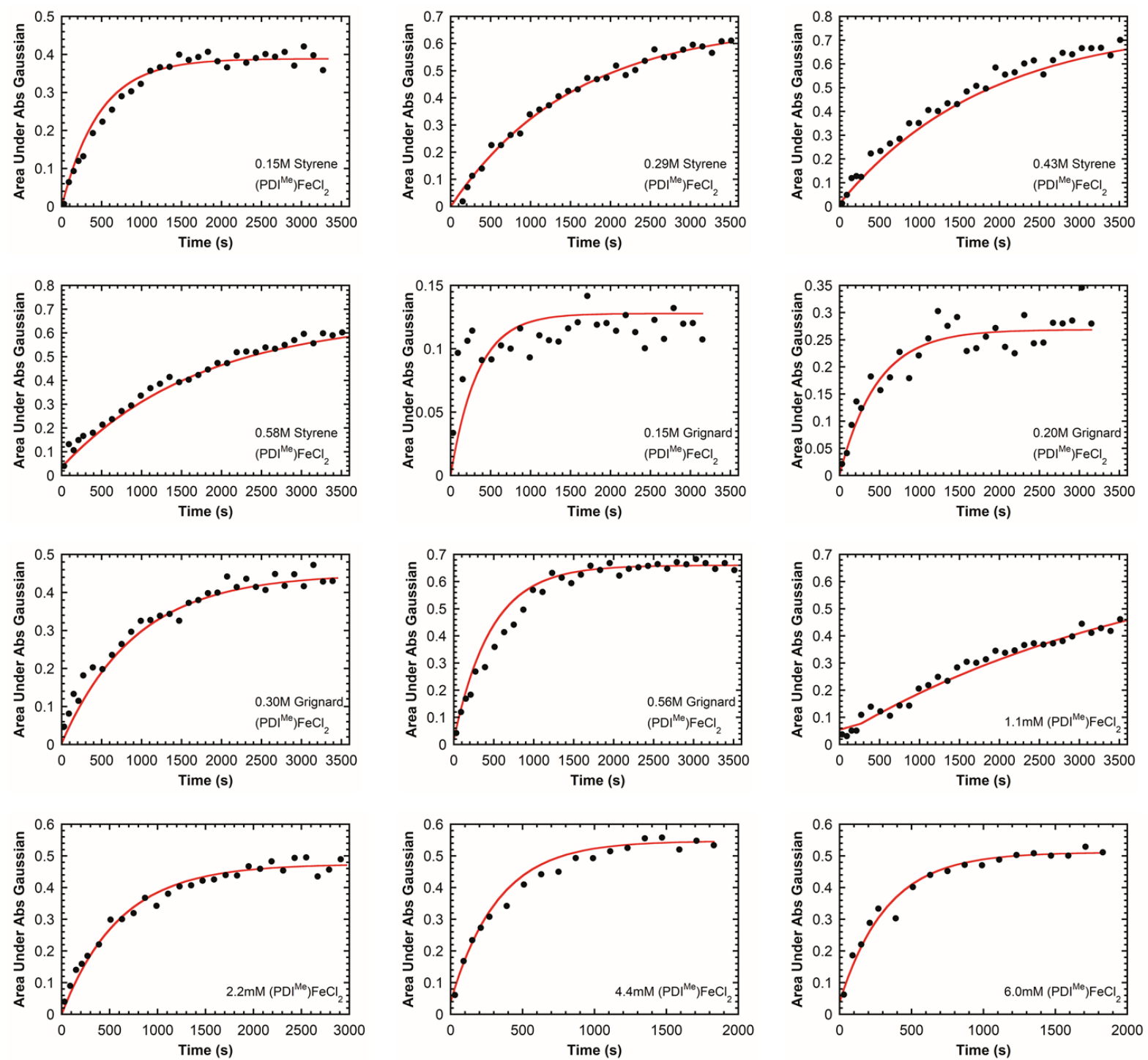

Figure E.18: [(1-phenylethyl)MgBr] vs time. Standard Conditions: 0.29 M S; $2.9 \mathrm{mM}$ $\left(\mathrm{PDI}^{\mathrm{Me}}\right) \mathrm{FeCl}_{2} ; 0.434 \mathrm{M} \mathrm{G}^{\mathrm{CYP}} ; 1$ hour; THF; rt. *See Figure for derivative concentrations. 
b. $\left(\mathrm{PDI} \mathrm{2}^{2-\mathrm{Me}}\right) \mathrm{FeCl}_{2}$
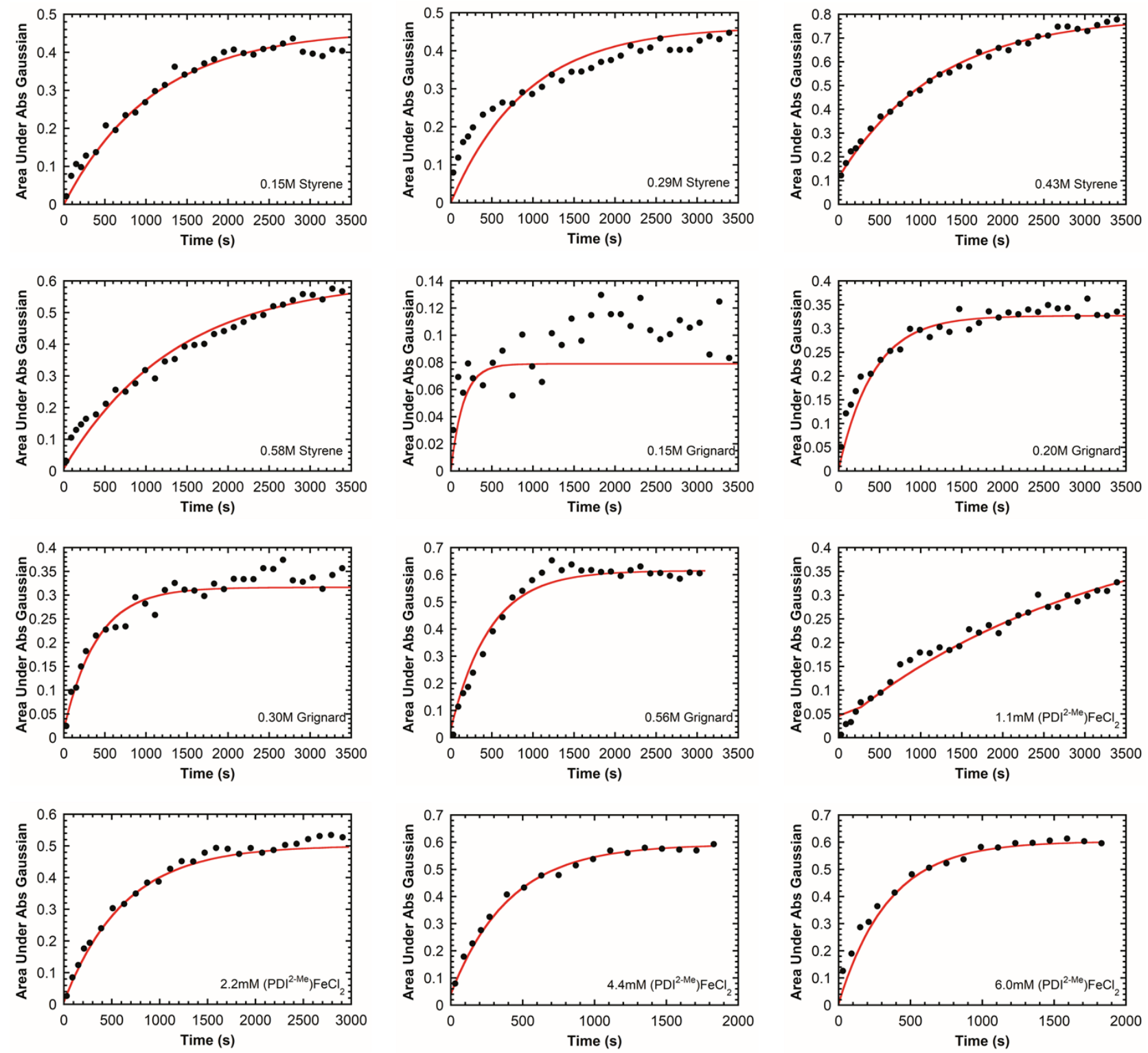

Figure E.19: [(1-phenylethyl)MgBr] vs time. Standard Conditions: 0.29 M S; $2.9 \mathrm{mM}$ $\left(\mathrm{PDI}^{2-\mathrm{Me}}\right) \mathrm{FeCl}_{2}$; $0.434 \mathrm{M}$ GCYP; 1 hour; THF; rt. *See Figure for derivative concentrations. 
c. $\left.(\mathrm{PDI})^{\mathrm{H}}\right) \mathrm{FeCl}_{2}$
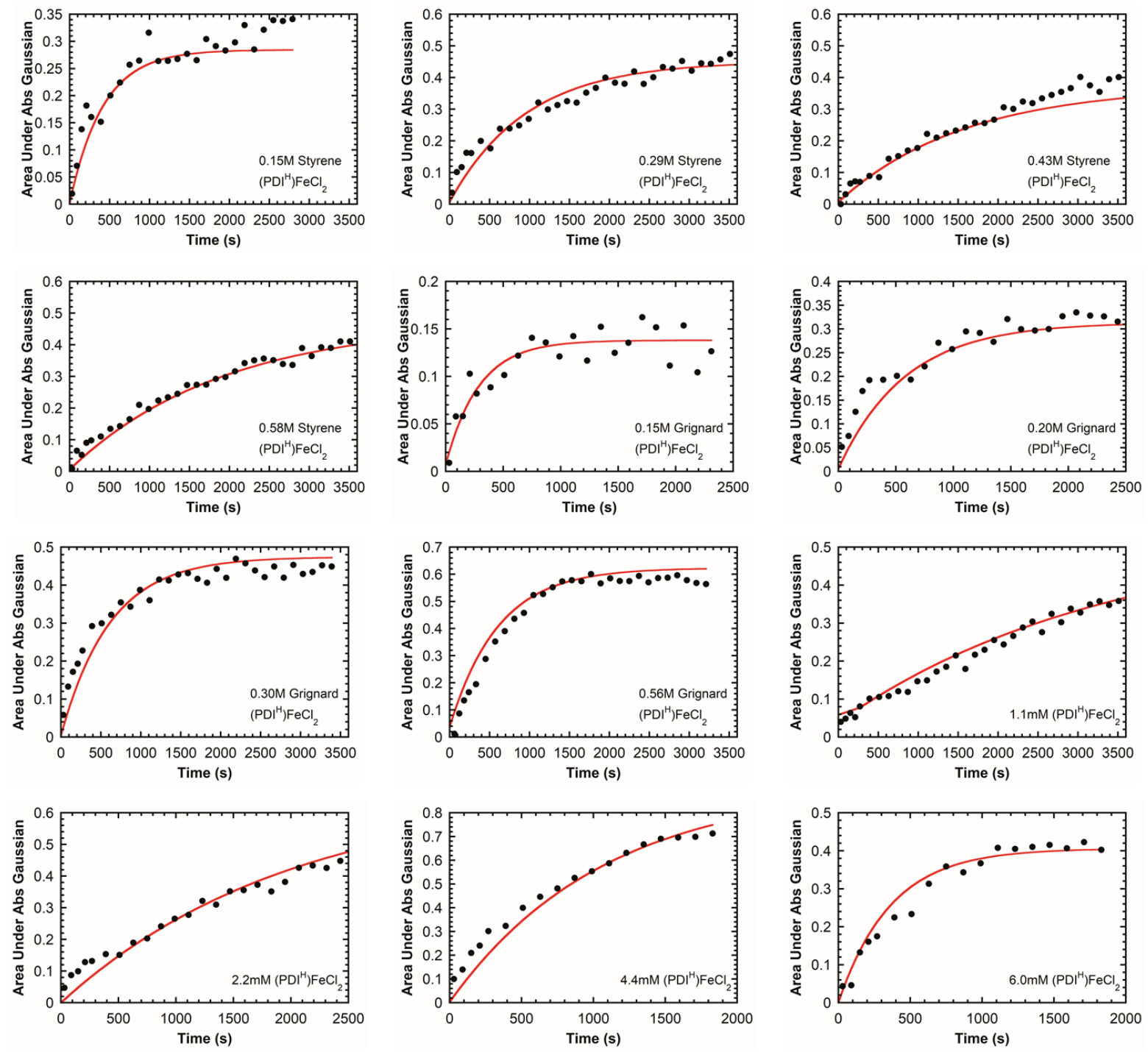

Figure E.20: [(1-phenylethyl) $\mathrm{MgBr}]$ vs time. Standard Conditions: $0.29 \mathrm{M} \mathrm{S} ; 2.9 \mathrm{mM}$ $\left(\mathrm{PDI}^{\mathrm{H}}\right) \mathrm{FeCl}_{2} ; 0.434 \mathrm{M} \mathrm{G}^{\mathrm{CYP}}$; 1 hour; THF; rt. *See Figure for derivative concentrations.

\section{COPASI Simulations}


General procedure of parameter estimation and time course estimation:

Kinetic models were constructed with the COPASI software (version 4.24). ${ }^{196,217}$ The initial concentrations used in the simulations were those reported in the experimental tables above. The rate constants were estimated in COPASI using the evolutionary programming method. The model accuracy is based upon the deterministic time course simulation using the LSODA algorithm. The agreement between experimental and numerically modelled timecourse data was assessed using Cohen's $\kappa$. Expected agreement for calculations is $50 \% .{ }^{218}$

\begin{tabular}{|c|c|}
\hline \multicolumn{2}{|r|}{$\begin{array}{c}\text { Cohen's Kappa for agreement } \\
\text { observed } \% \text { - expected } \%\end{array}$} \\
\hline$\kappa<0$ & Minimal agreement \\
\hline $0.1<\kappa<0.2$ & Slight agreement \\
\hline $0.21<\kappa<0.4$ & Fair agreement \\
\hline $0.41<\kappa<0.6$ & Moderate agreement \\
\hline $0.61<\kappa<0.8$ & Substantial agreement \\
\hline $0.81<\kappa<0.99$ & Near-perfect /excellent agreement \\
\hline$\kappa=1$ & Perfect agreement \\
\hline
\end{tabular}




\section{Experimental Appendix II Contents:}

1. ReactIR set up and tutorial

2. IGOR tutorial and procedure files

3. COPASI tutorial 


\section{ReactIR Tutorial and Training:}

The following steps are to ensure proper usage. Slowly fill the detector with 1 full dewar of liquid nitrogen then allow instrument to equilibrate for 20 minutes. Open iC IR 7.0 software and follow the instructions below.

1. Click on Instrument

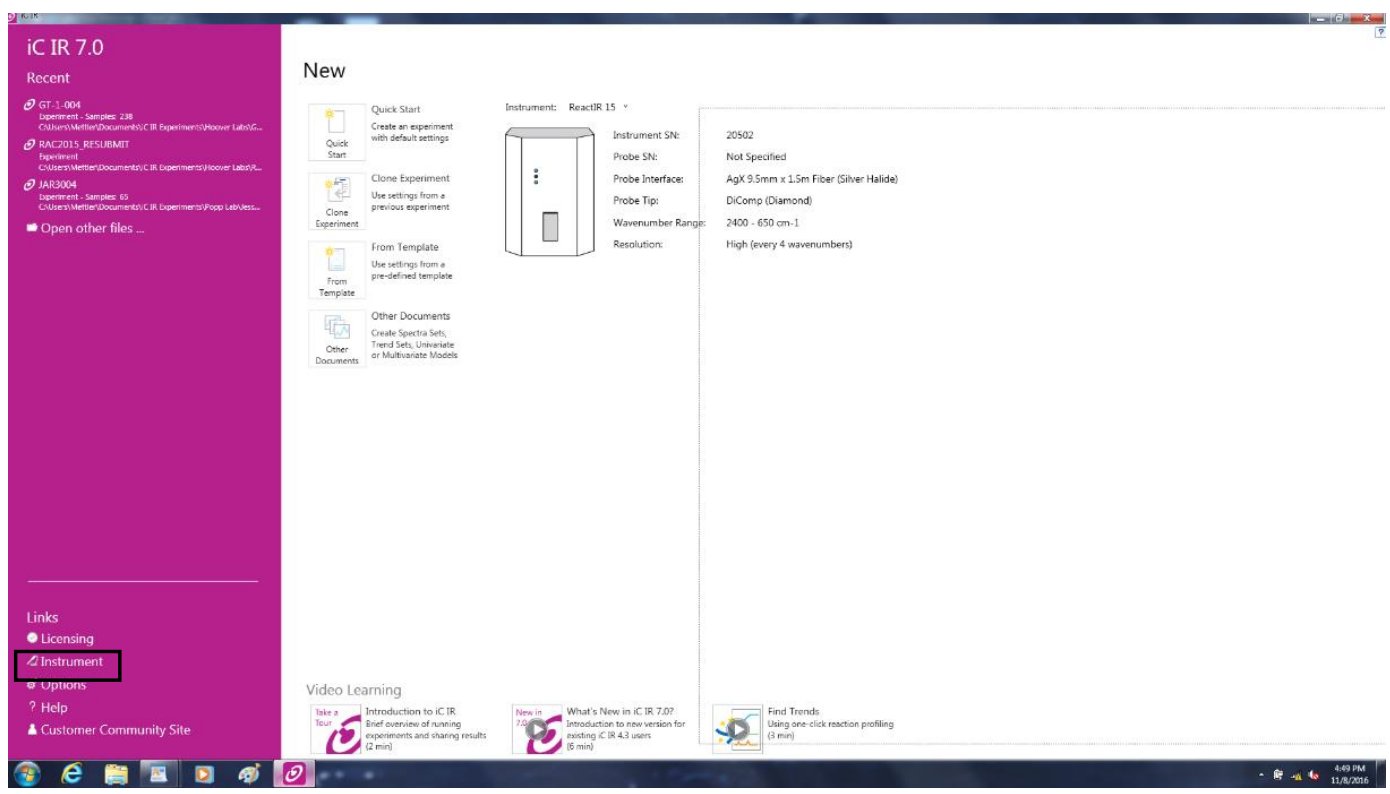

a. Click on configure

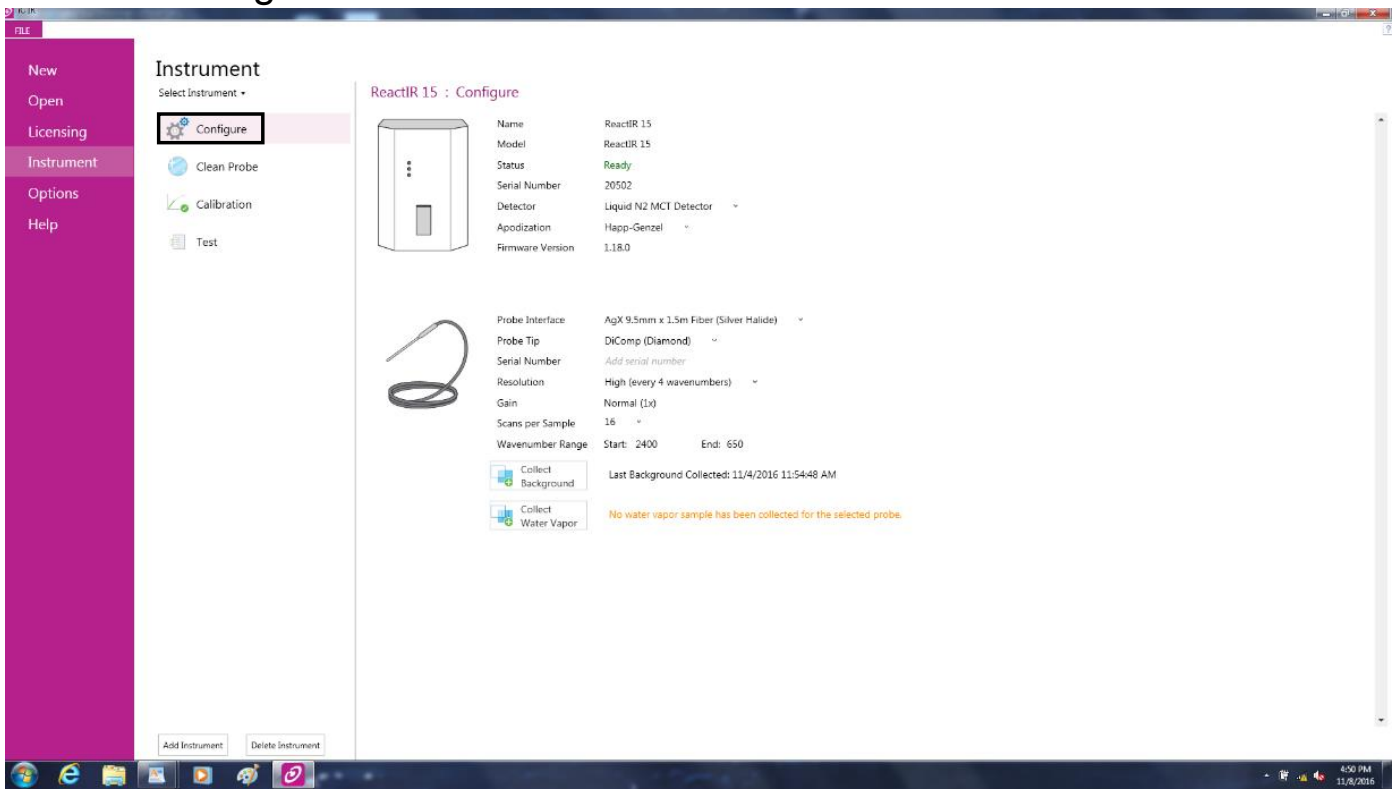

i. Under configuration menu

1) Status should say Ready

2) Change Probe Interface 
a. Choose one of the two highlighted dependent upon the probe you will be using.

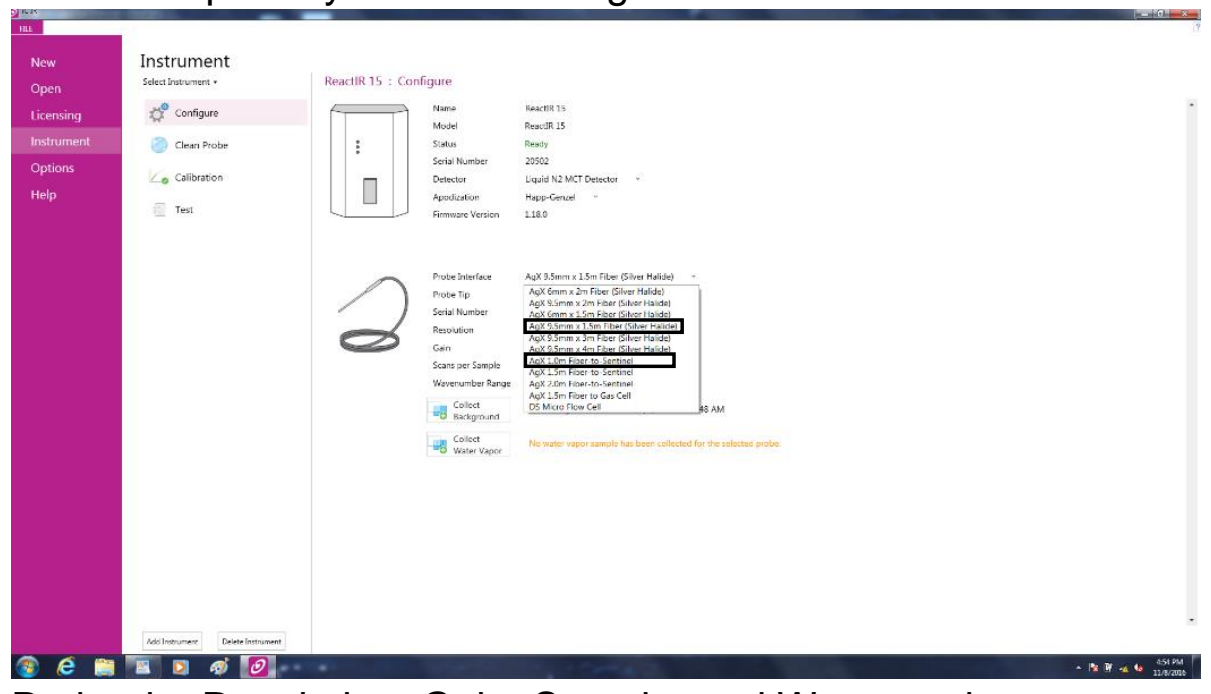

3) Probe tip, Resolution, Gain, Sample, and Wavenumber range should remain constant.

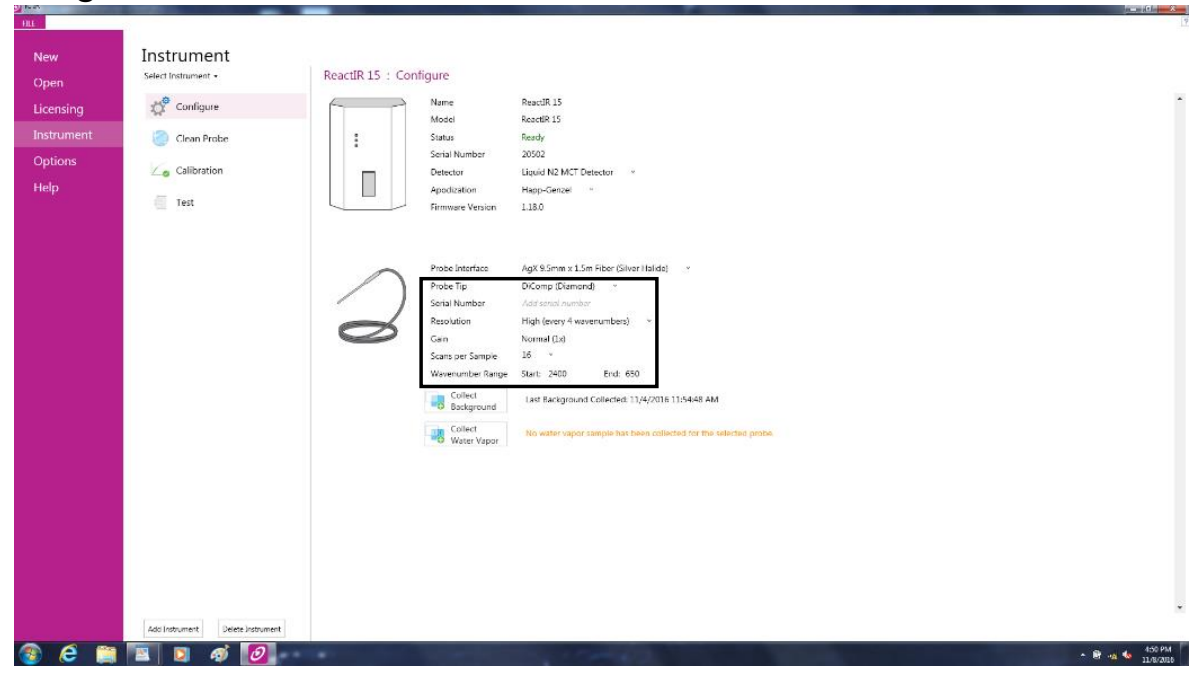

4) Collect Background 


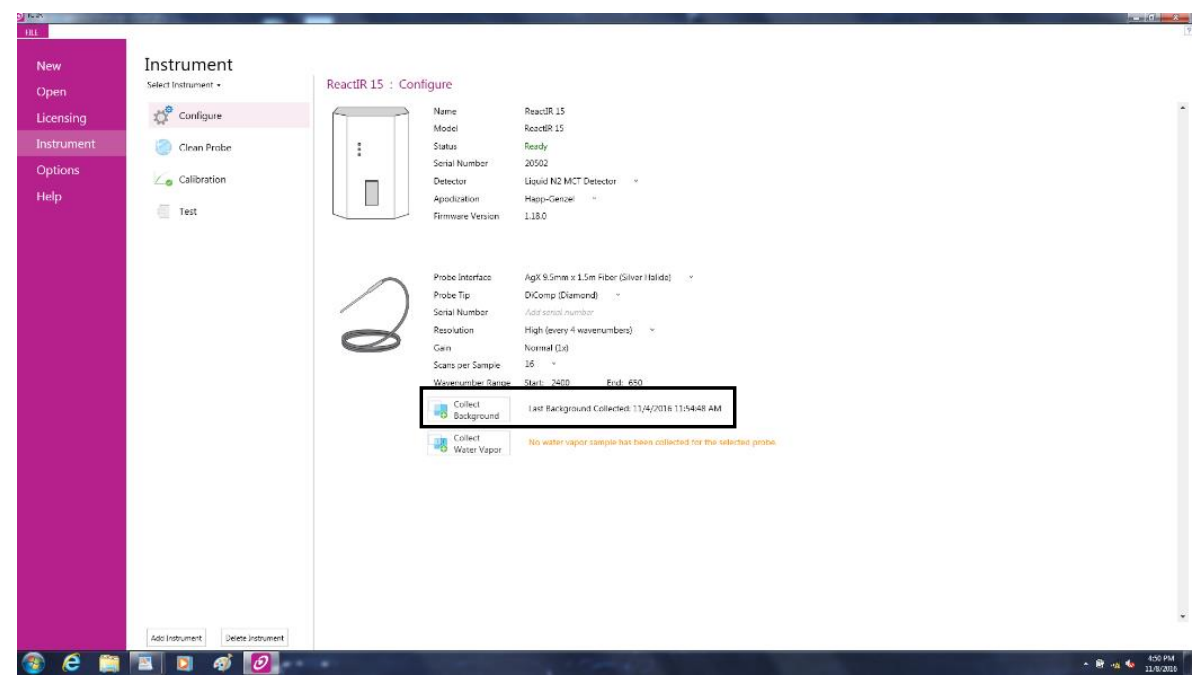

5) Click Continue when this window pops up as long as instrument alignment is in Green and MCT detector status is Green

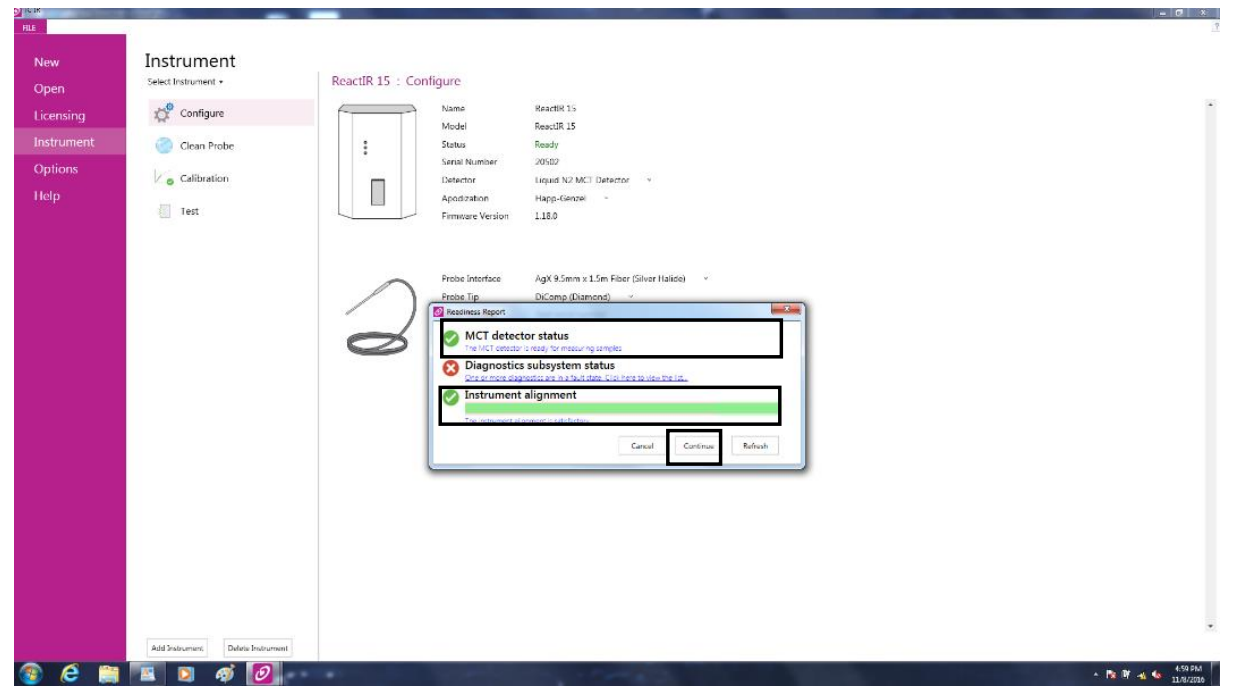

6) Collect Background, then Done

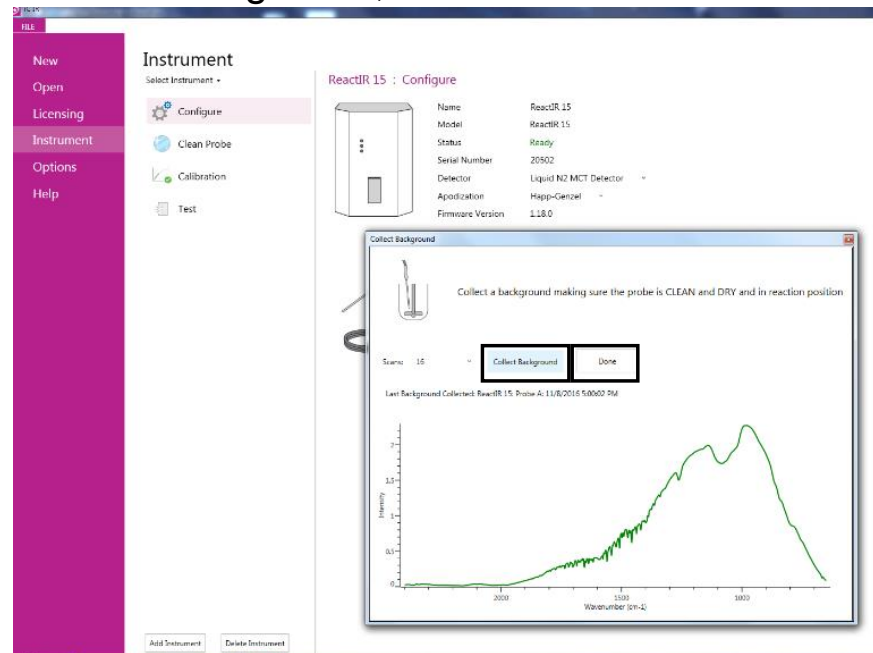


b. Click on clean Probe and Start

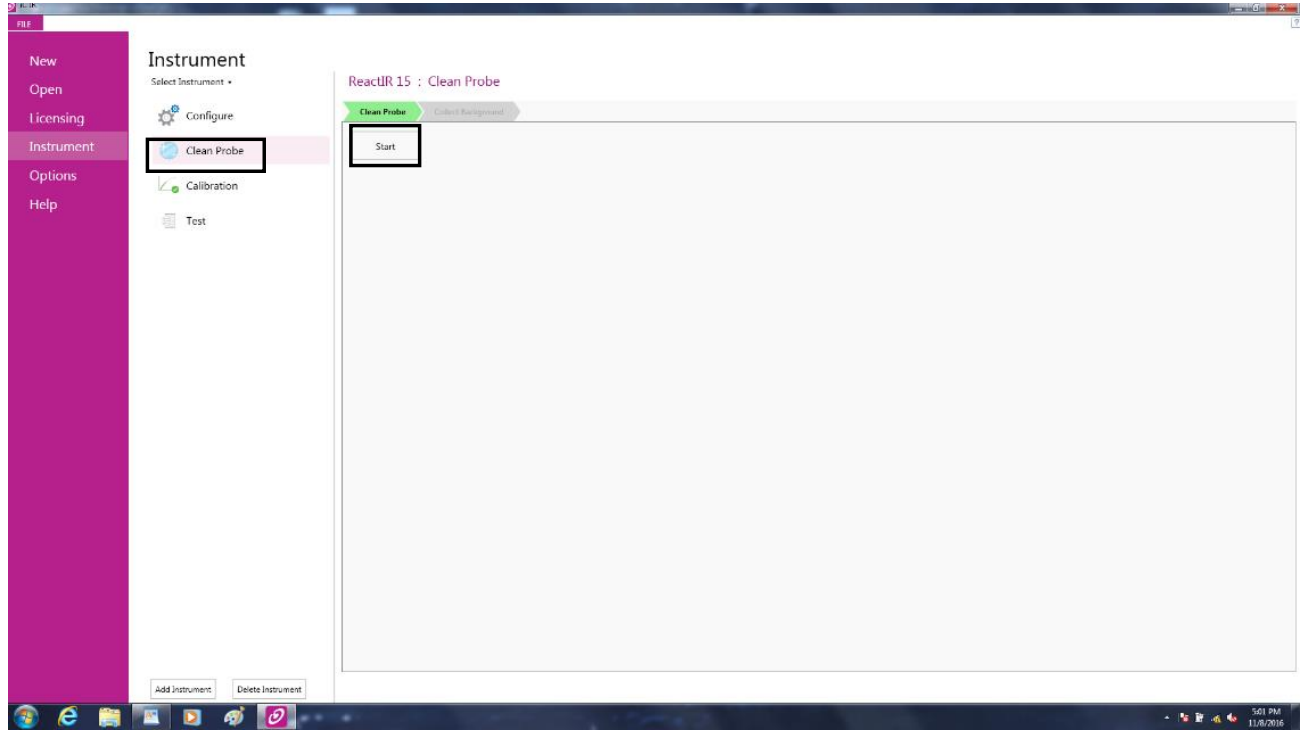

i. If in range and a flat line, then click Next

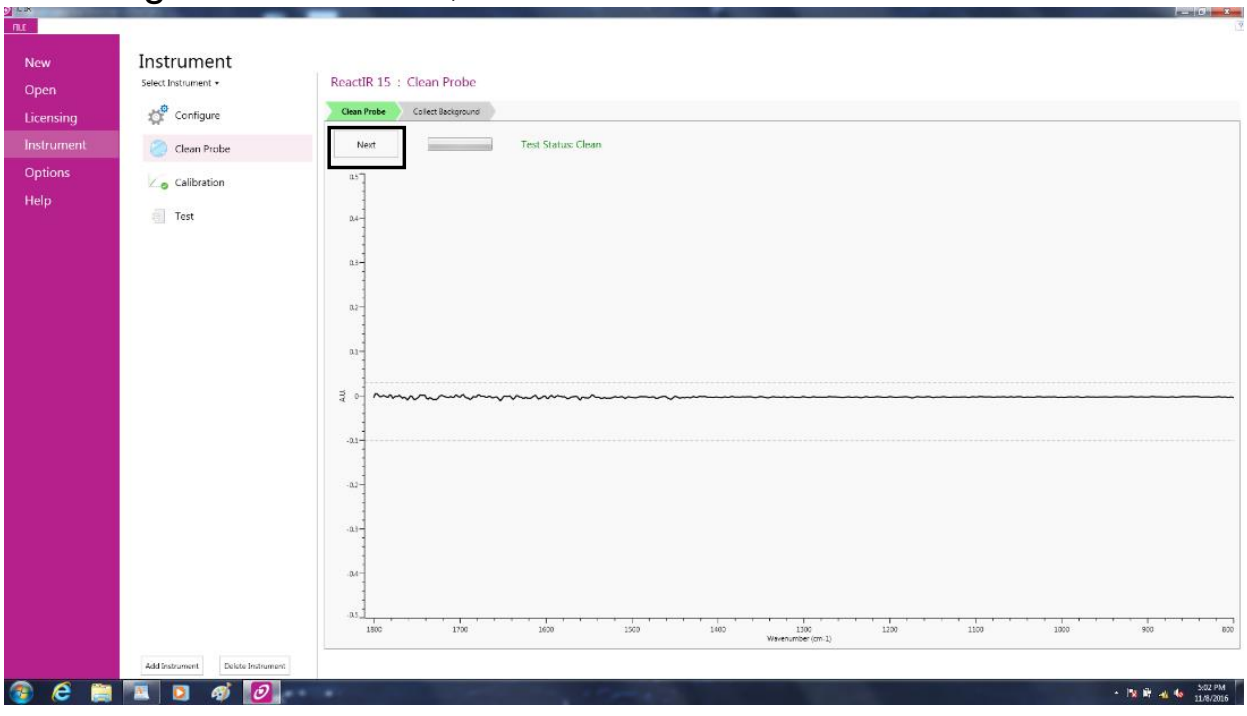

ii. Collect Background, then done 
2. Start a new experiment

a. File, then New, choose from Quick Start, Clone Experiment, From Template, Other documents.

i. Select Continue once you select your experiment type. In the manual we will start with Quick Start.

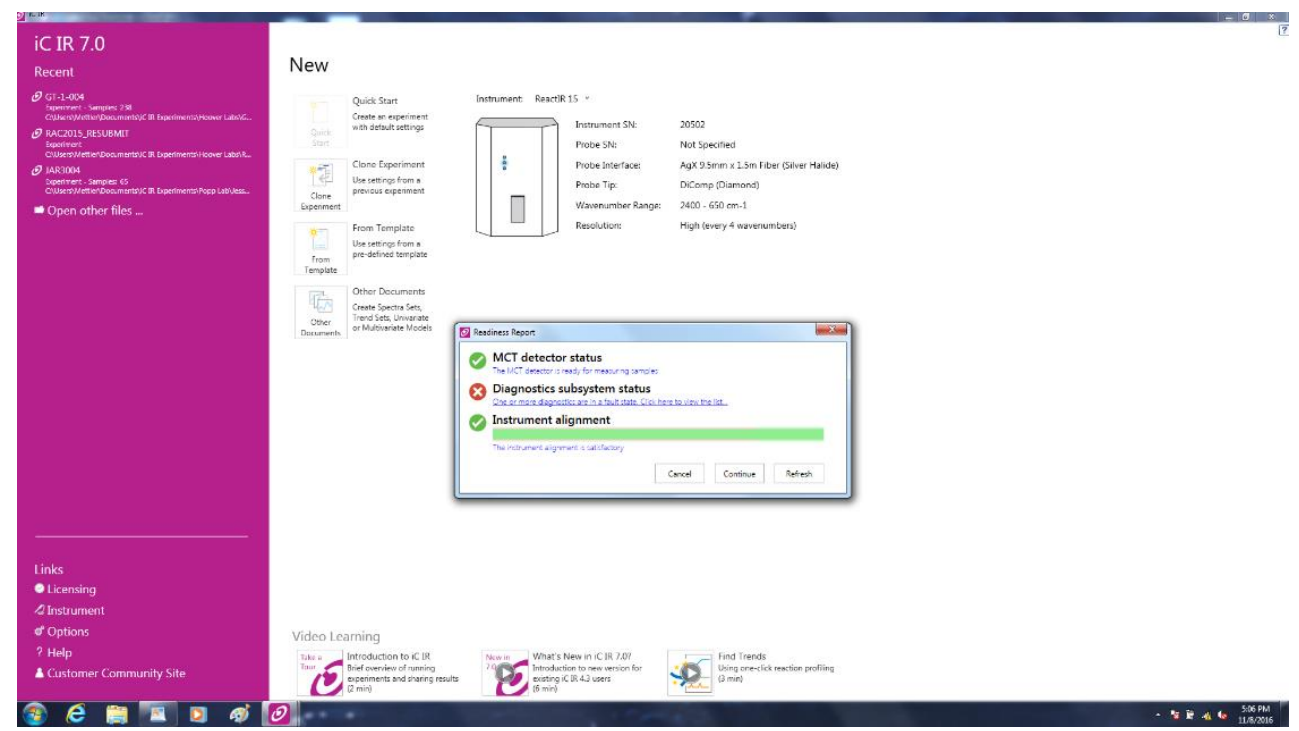

ii. Name your file and determine the file folder to save your document. Then select your duration and scan per sample. Click Create

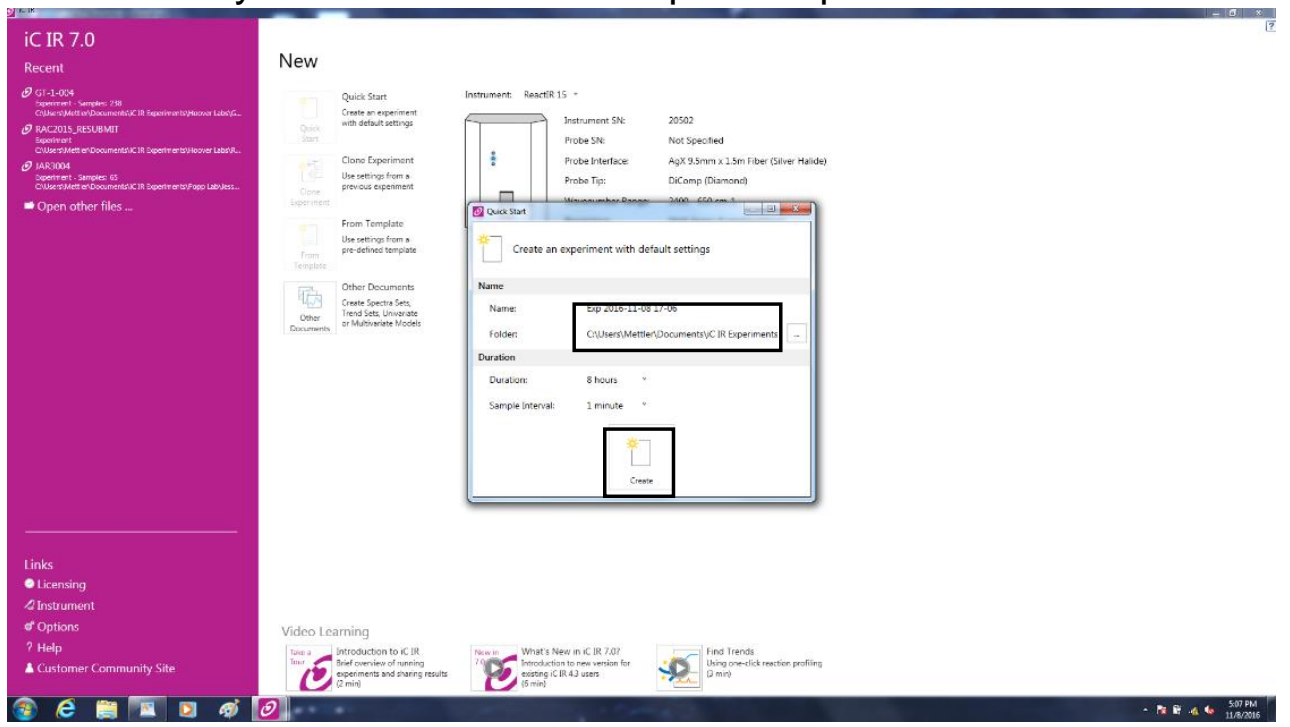


iii. To add more to your duration time, select Phase.

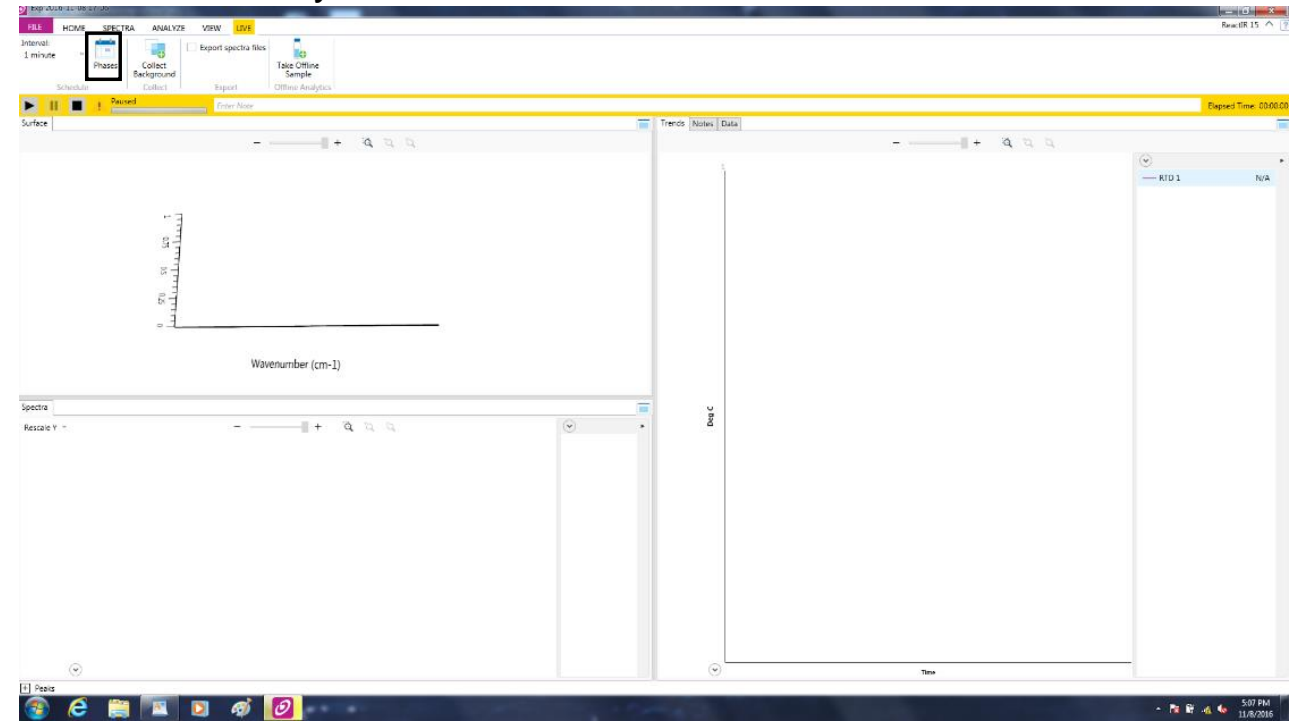

iv. Add phase time

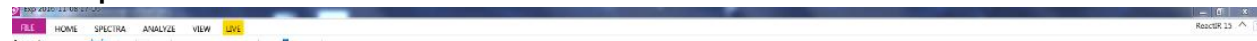

$=-\frac{1}{2}$
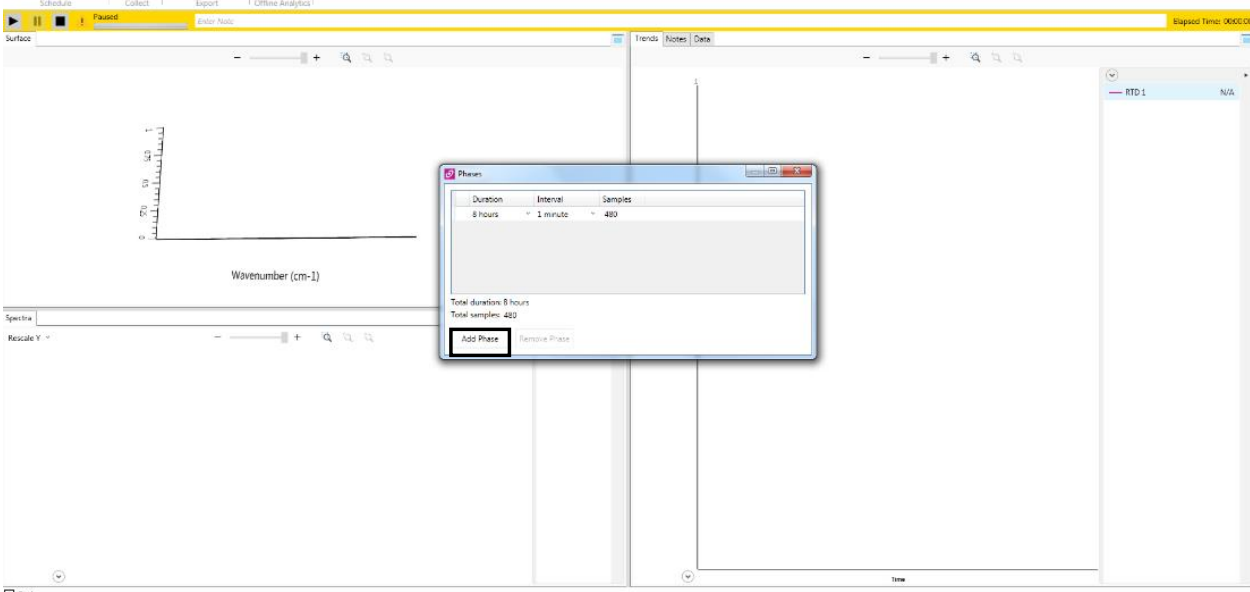

(9) e

v. To begin sample collection, press Play 
vi. Upon completion clean probe with $0.1 \mathrm{M} \mathrm{HCl}$, isopropanol, and acetone. Click Next and collect new background

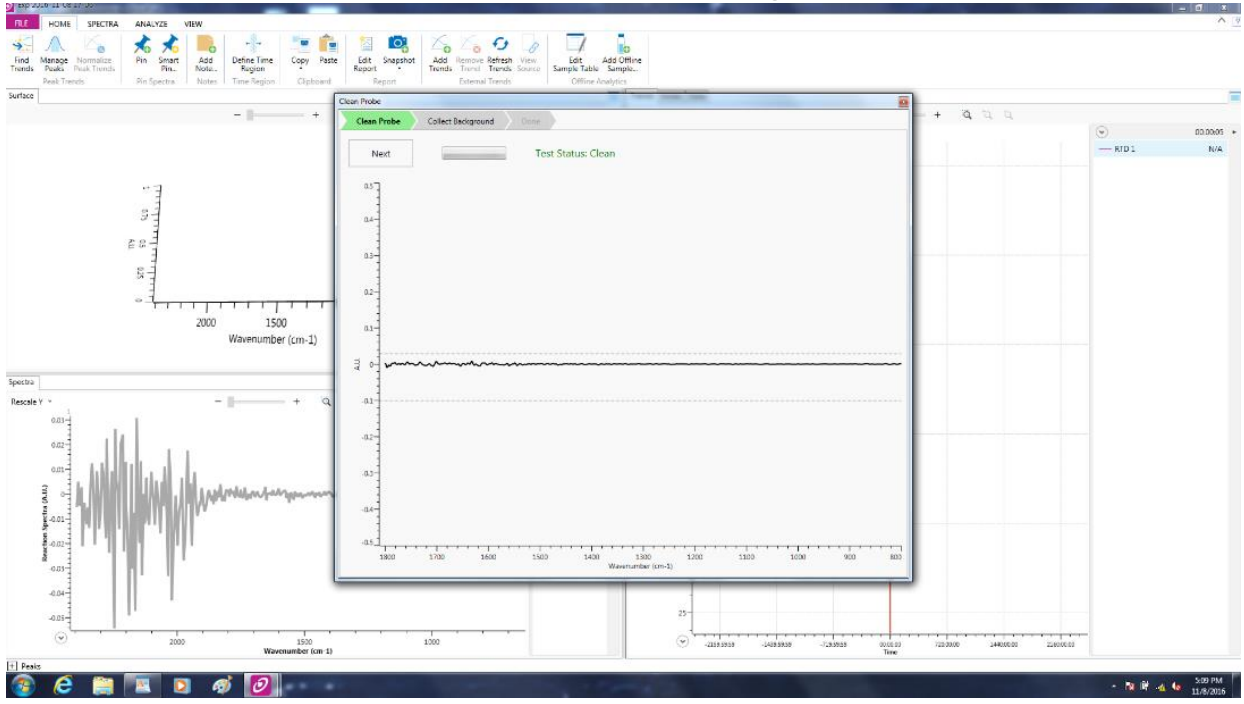

3. Collecting References

a. File, New, Other Documents. Select Spectra Set.

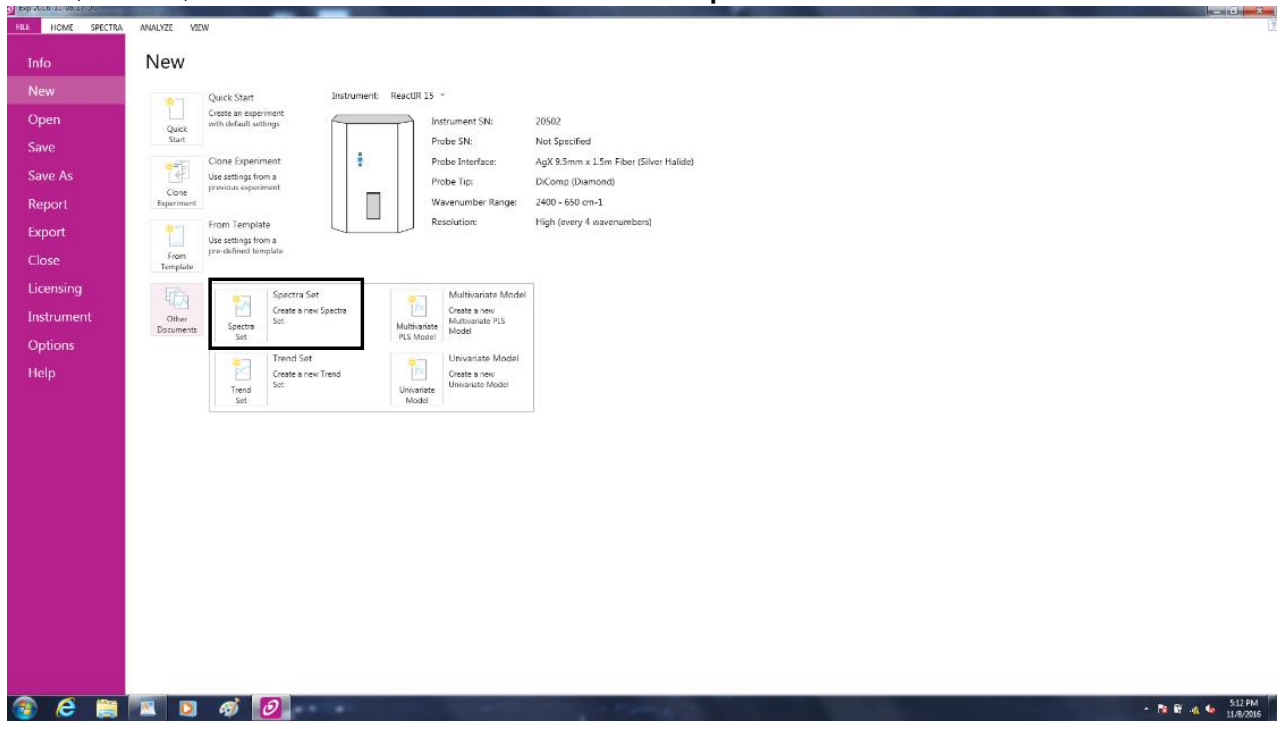


b. Click on collect reference

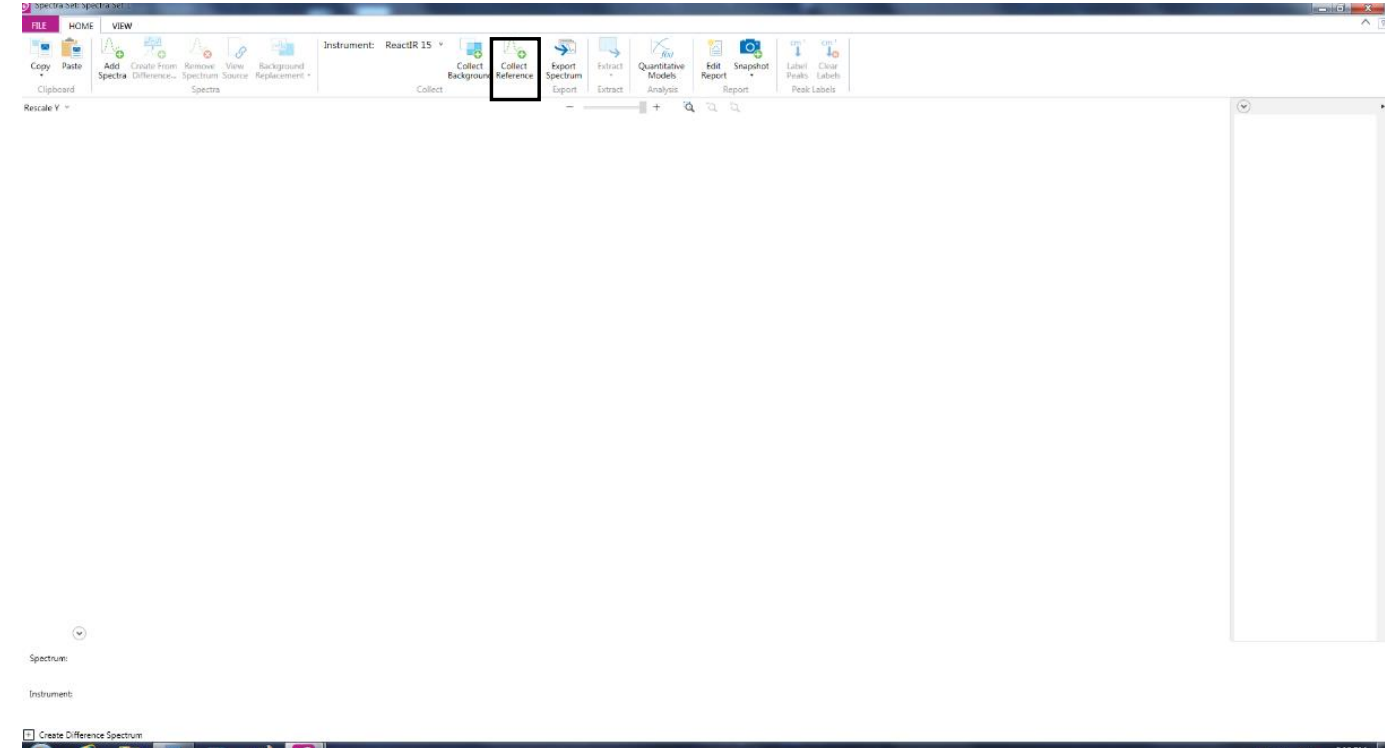

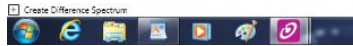

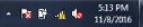

c. Collect a background or use a previous background.

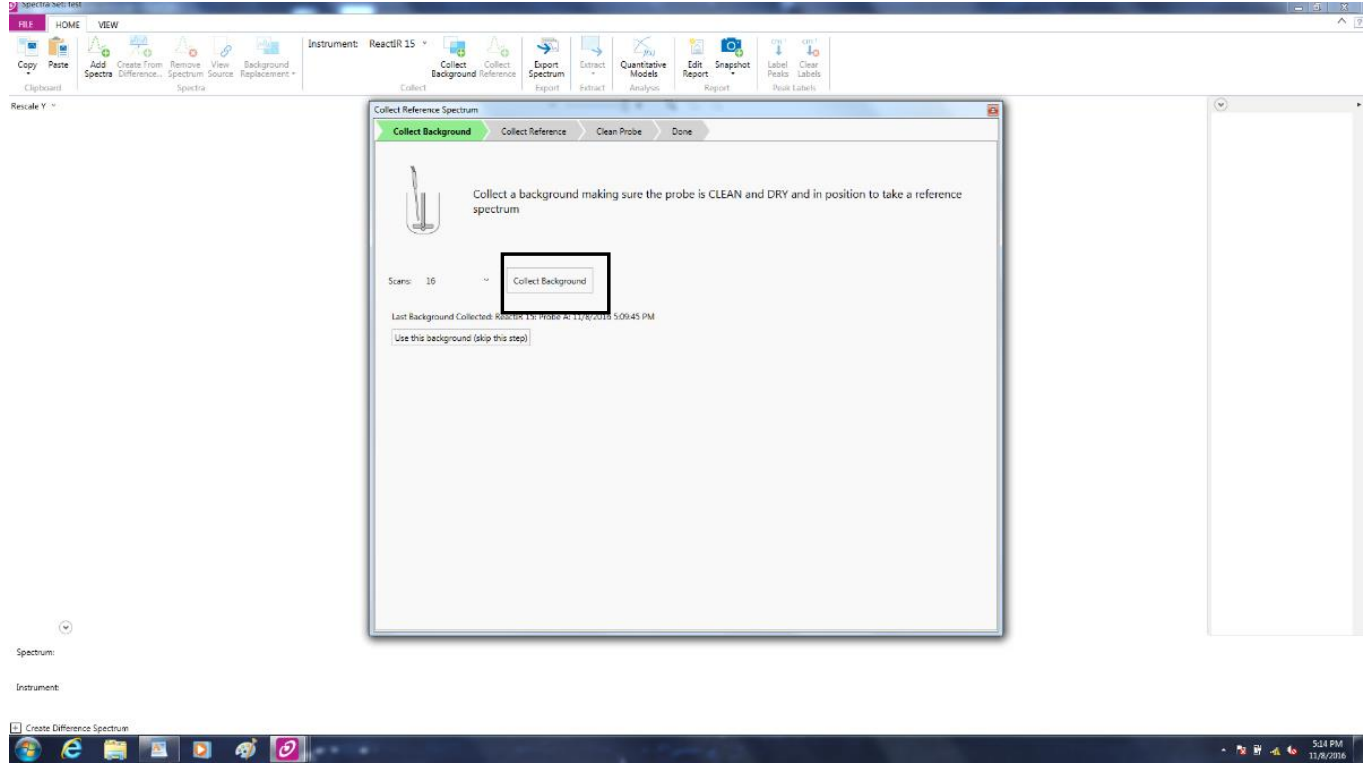

-T9- 
d. Type in the name of the reference and choose from type and functional groups. They have dropdown menus.

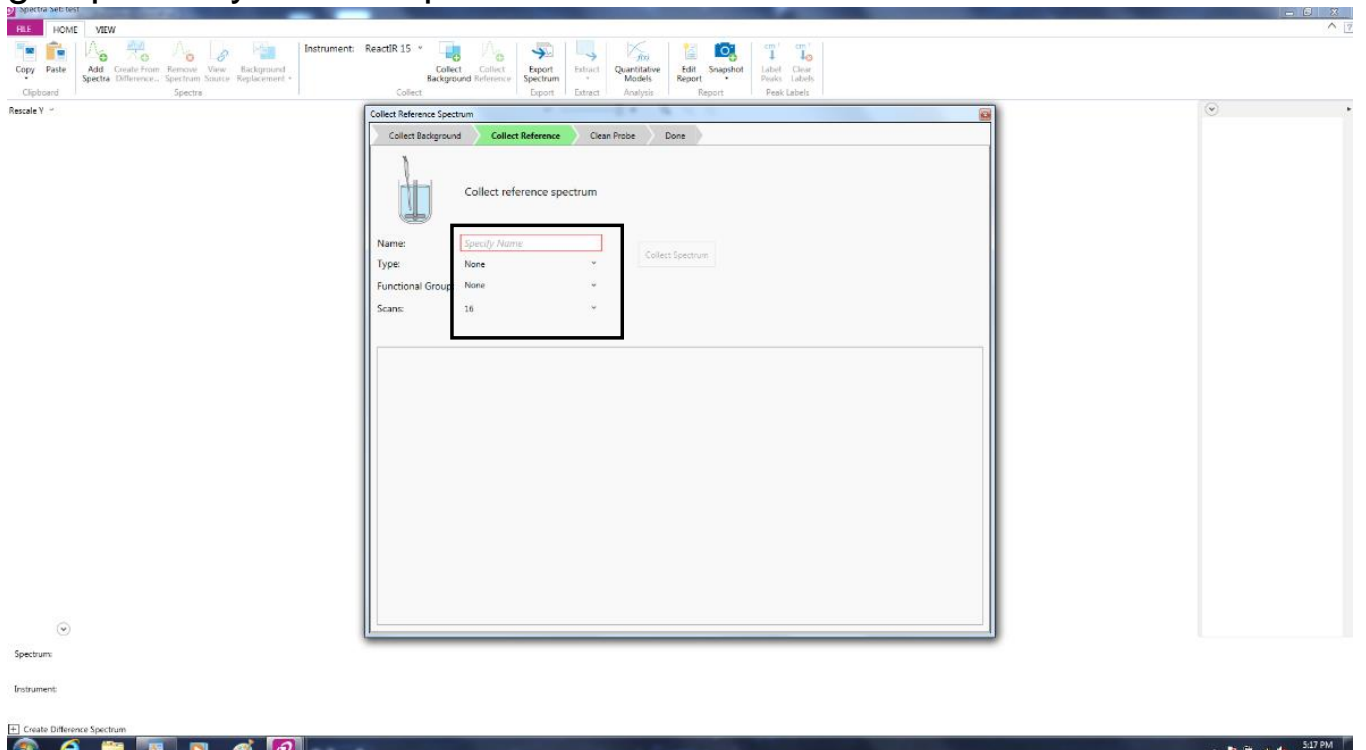

e. Collect the reference.

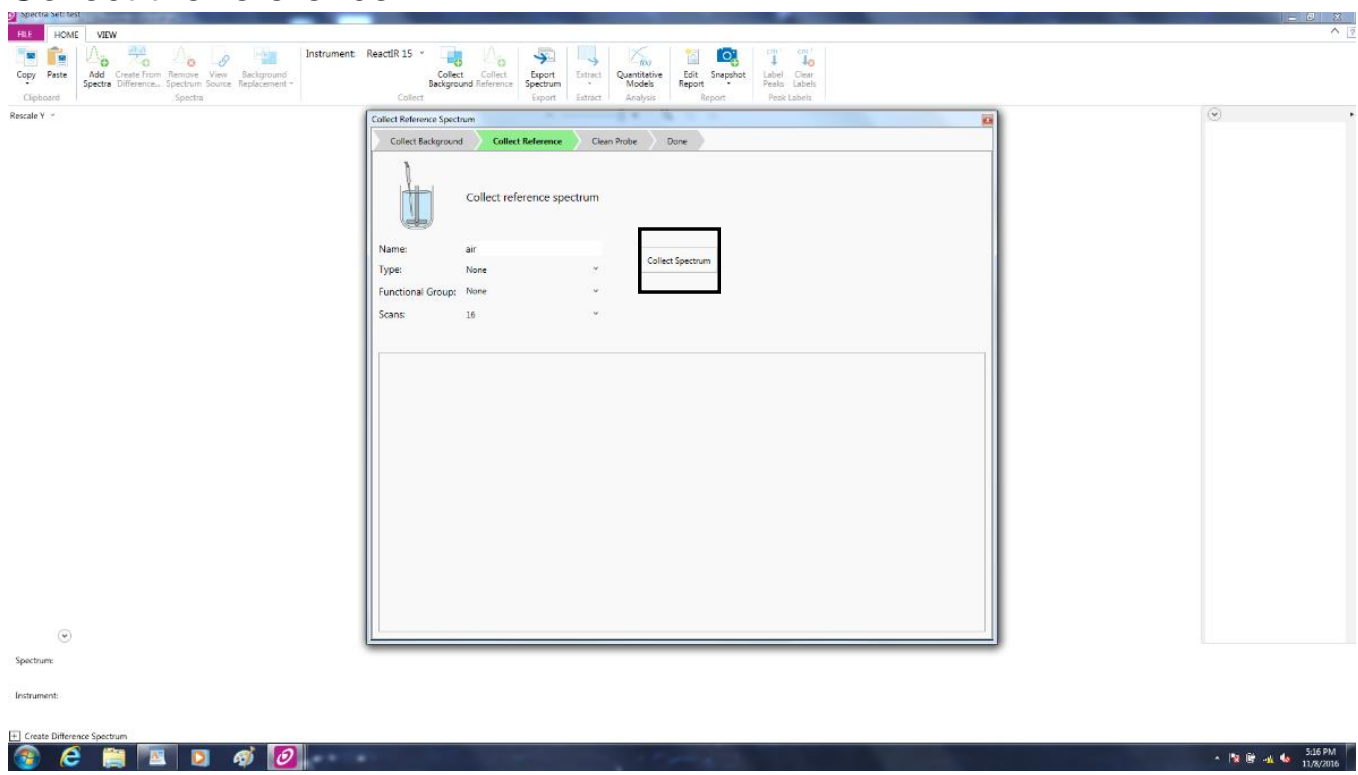


f. When finished click next.

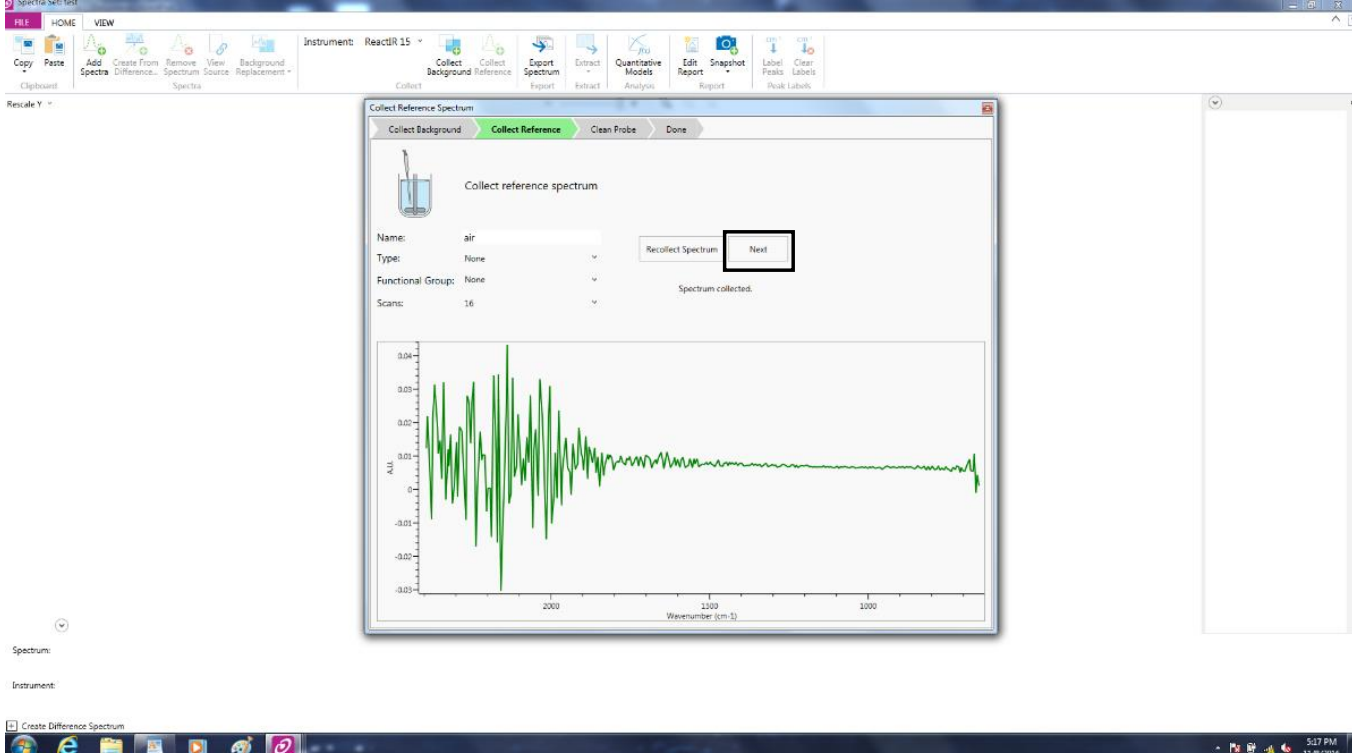

g. Clean the probe and click done.

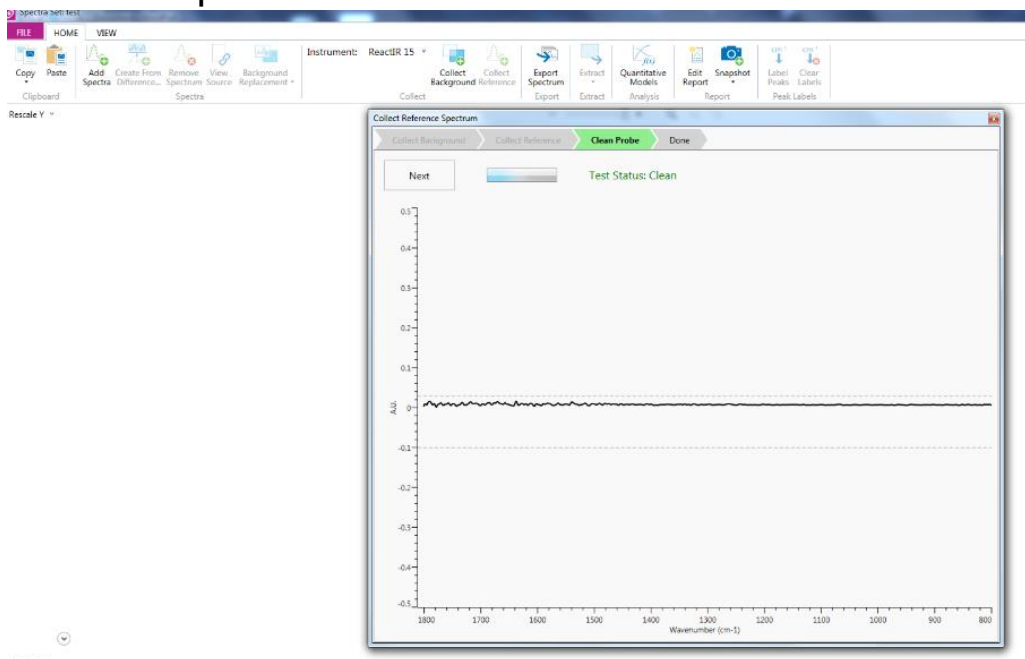

h. Repeat steps b-g as needed for References.

i. When running the experiment to add references click on add spectra in upper left and follow prompts.

- If you already have an experiment where you have identified the trends or references, you may select clone experiment from the start new experiment menu. This will prompt you to select the experiment you want to clone and name the new file. The reset of the commands from quick start are the same. 
4. To export data from iC IR programming.

a. Under the surface tab. Highlight the surface area of or tip of curve you want to see a trend for.

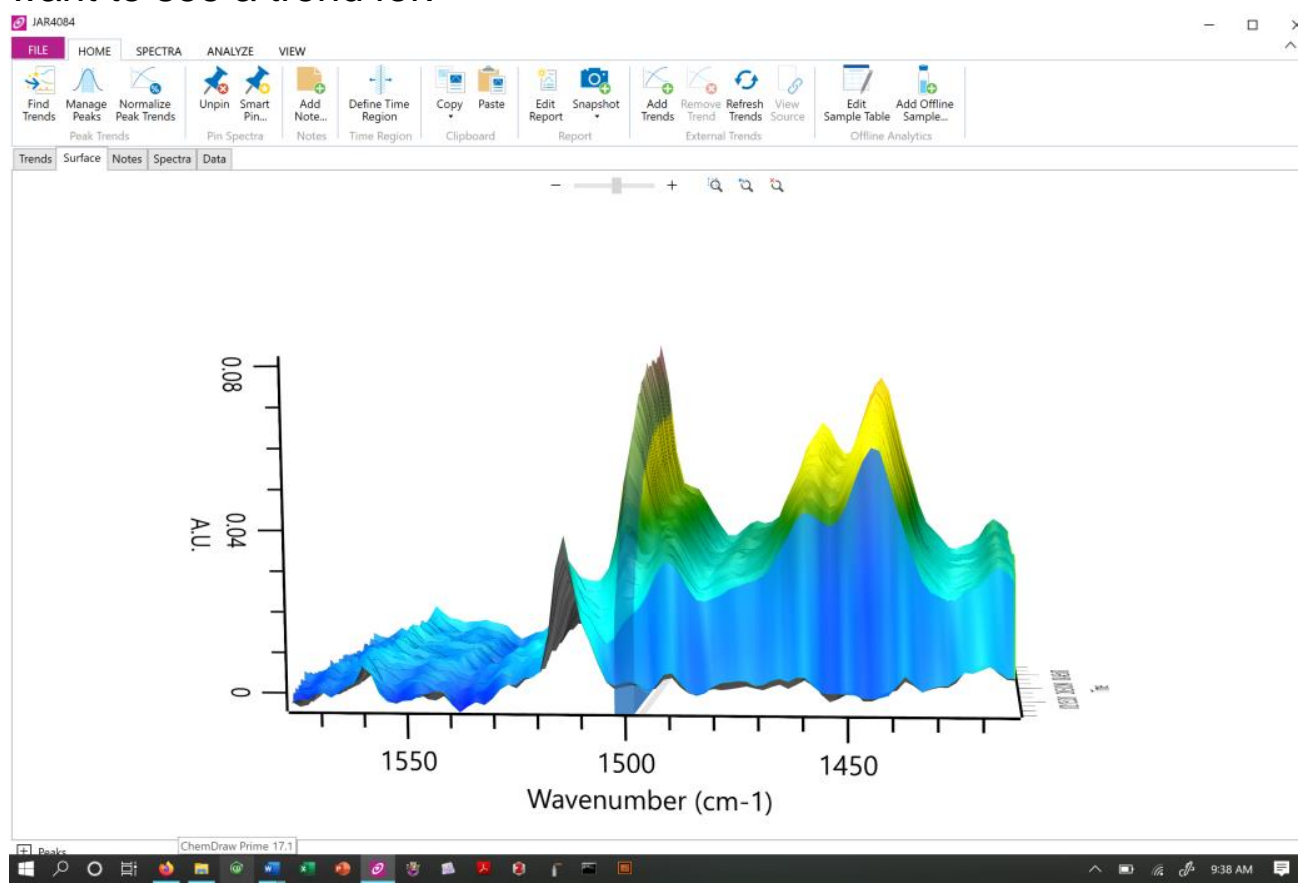

b. To subtract the solvent from the experimental data, select the spectra tab at the top. Using the drop down select one of the imported references.

G JARAO

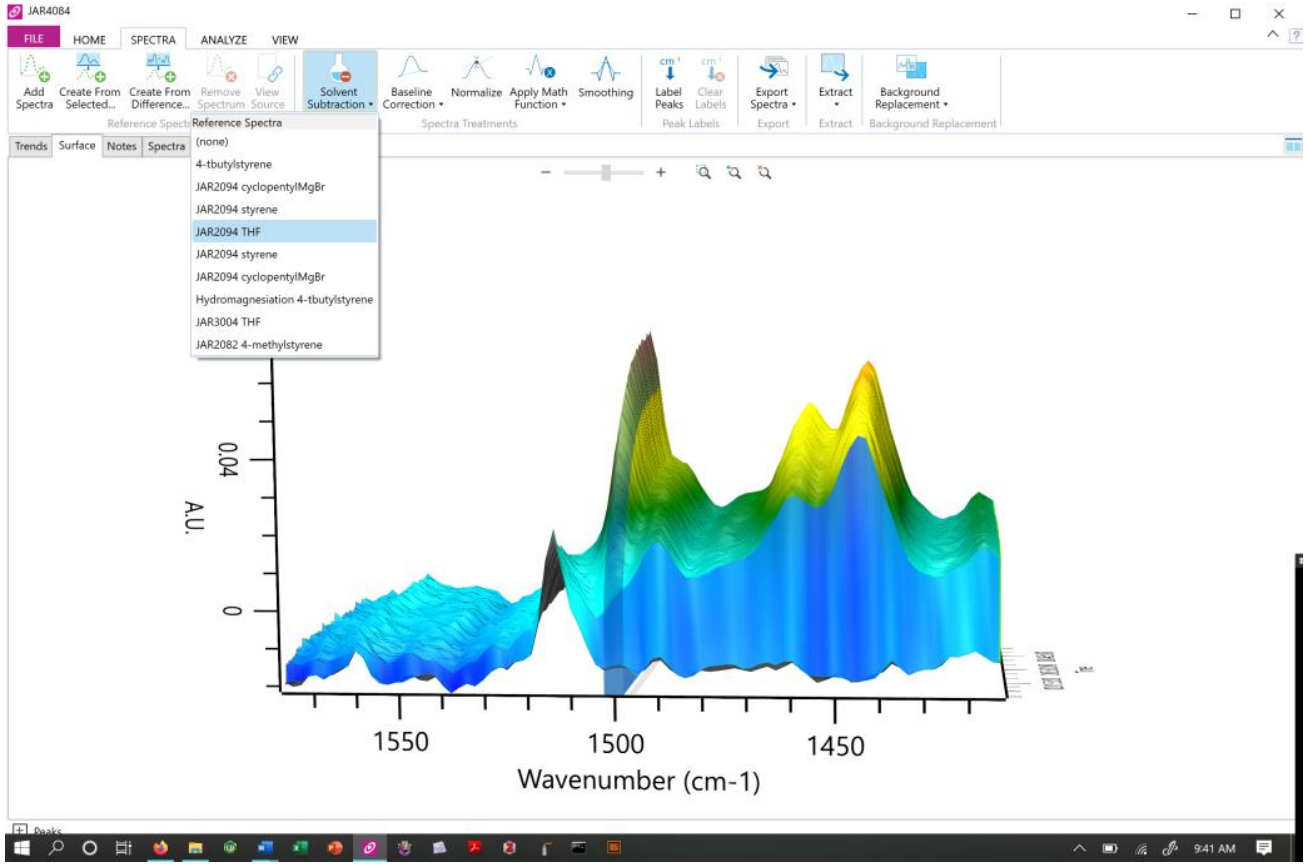

c. Under the trends tab the highlighted signals are shown in a plot of absorbance vs time. 
d. To begin exporting process under the home tab select "Smart Pin"

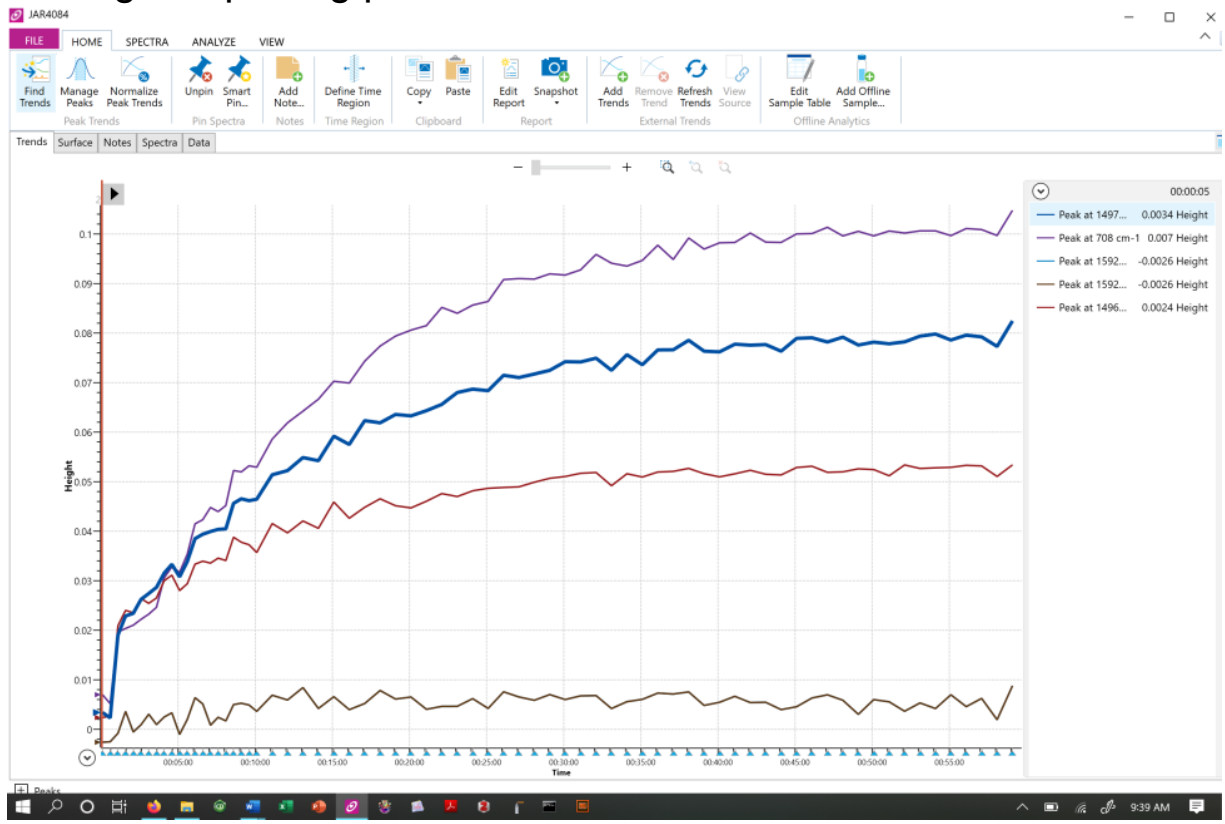

e. A new dropdown window will open.

i. Select "Pin every _ minutes"

ii. Type in a time where no more than 59 samples will be selected.

iii. Select "Pin _ samples"

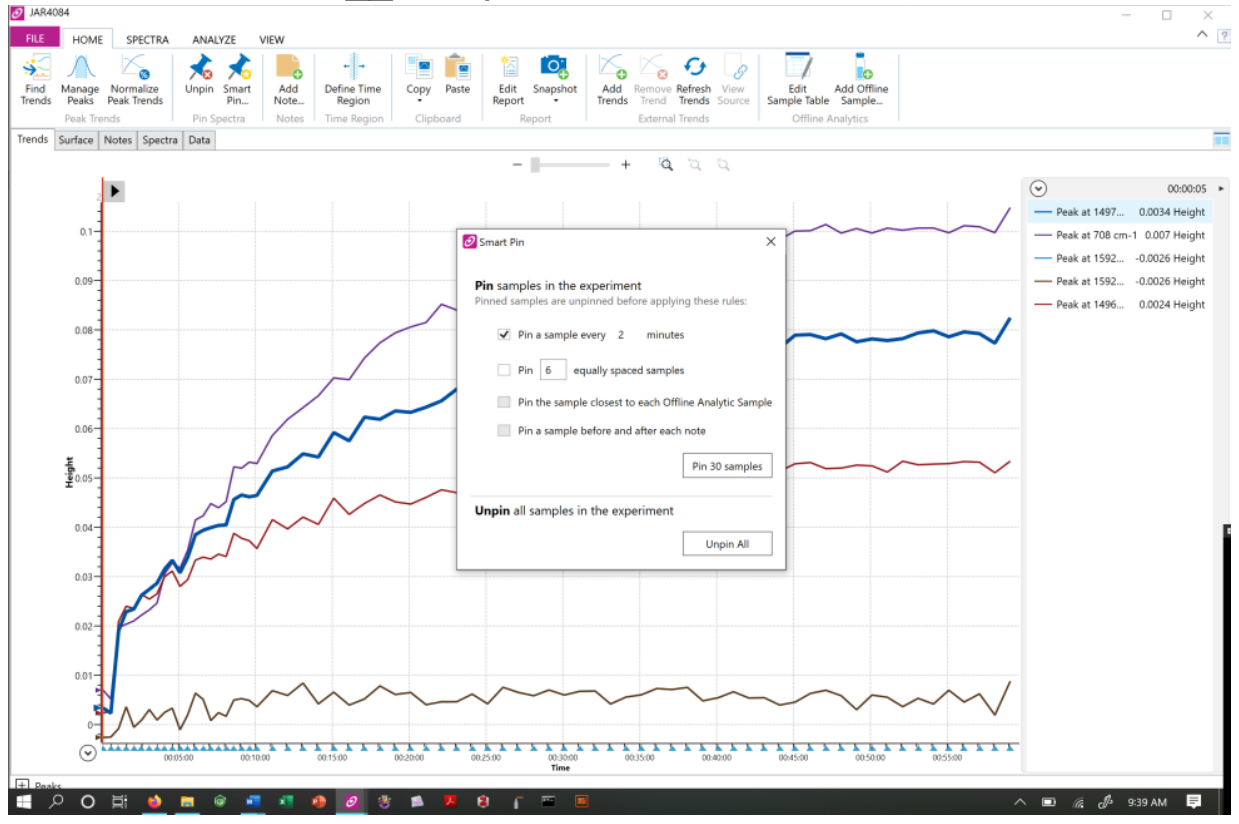


f. To pin any remaining collected spectra select the "Notes" tab and click on the gray thumb tacks. (When pinned the thumb tack will be blue). G JARAOBA

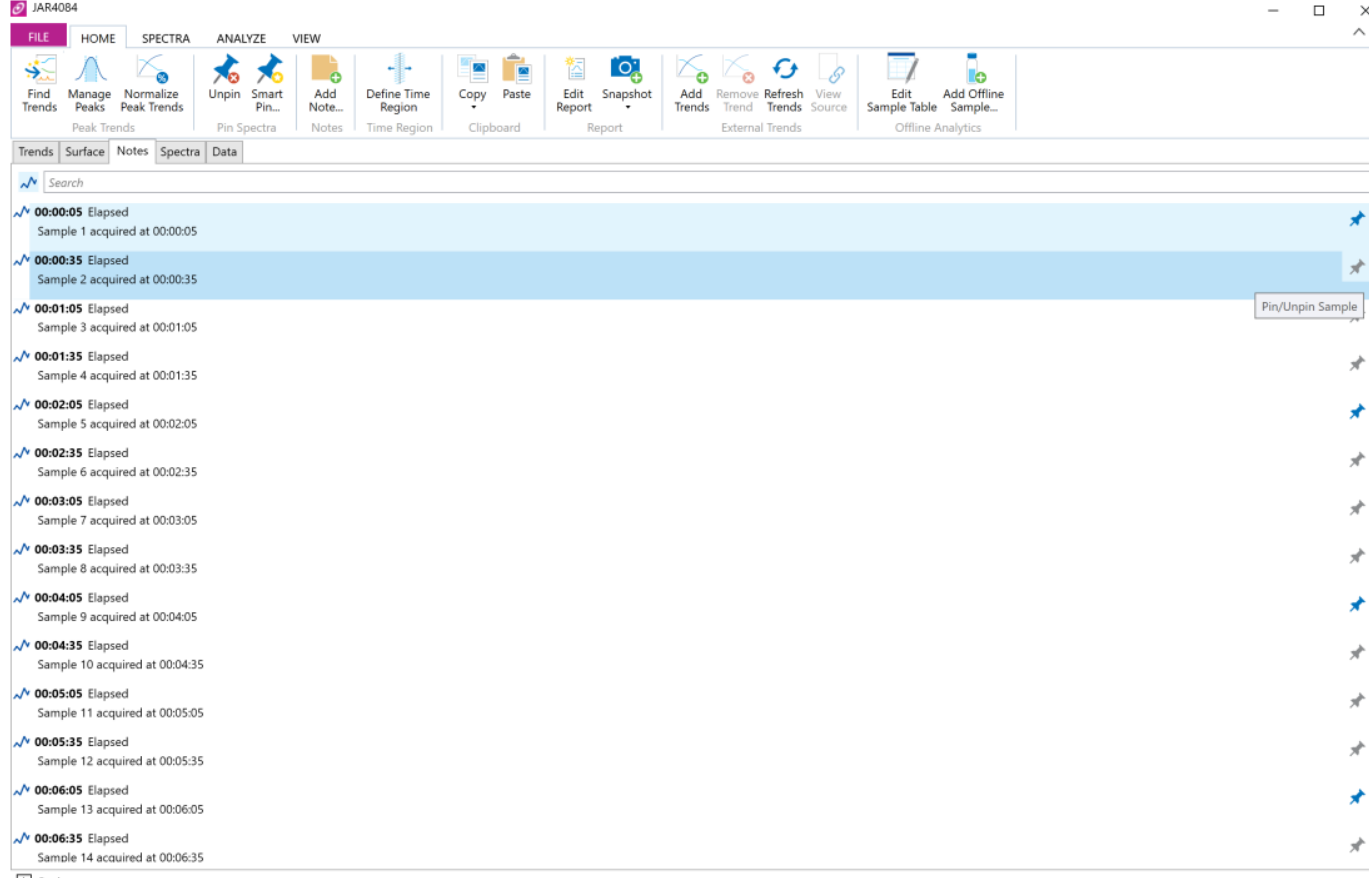

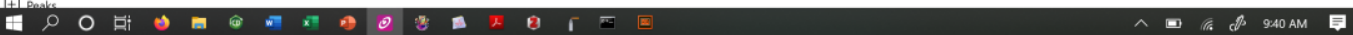

g. Once spectra are pinned under the "Spectra" tab select export spectra and all pinned spectra from the drop down.

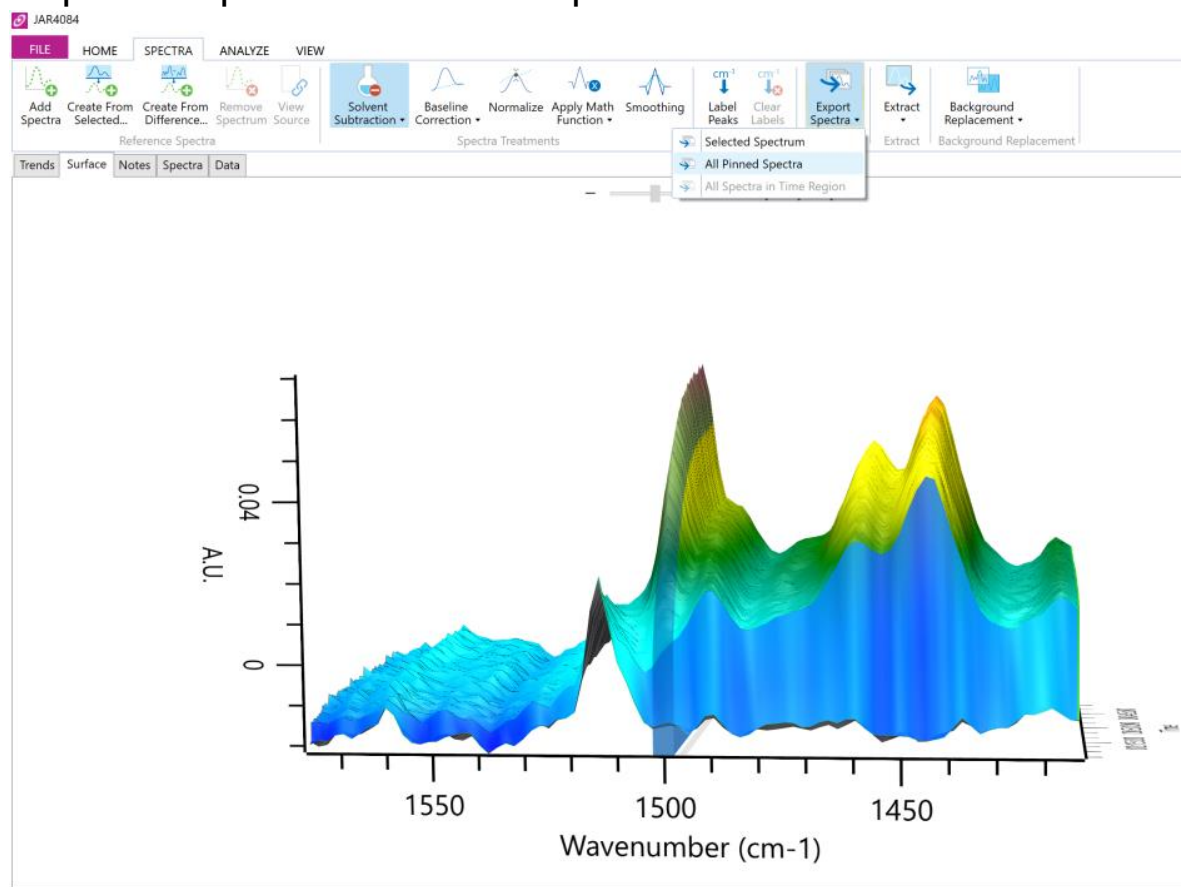


h. Under the export window select "Multi-spectrum file", CSV, Treated, then export.

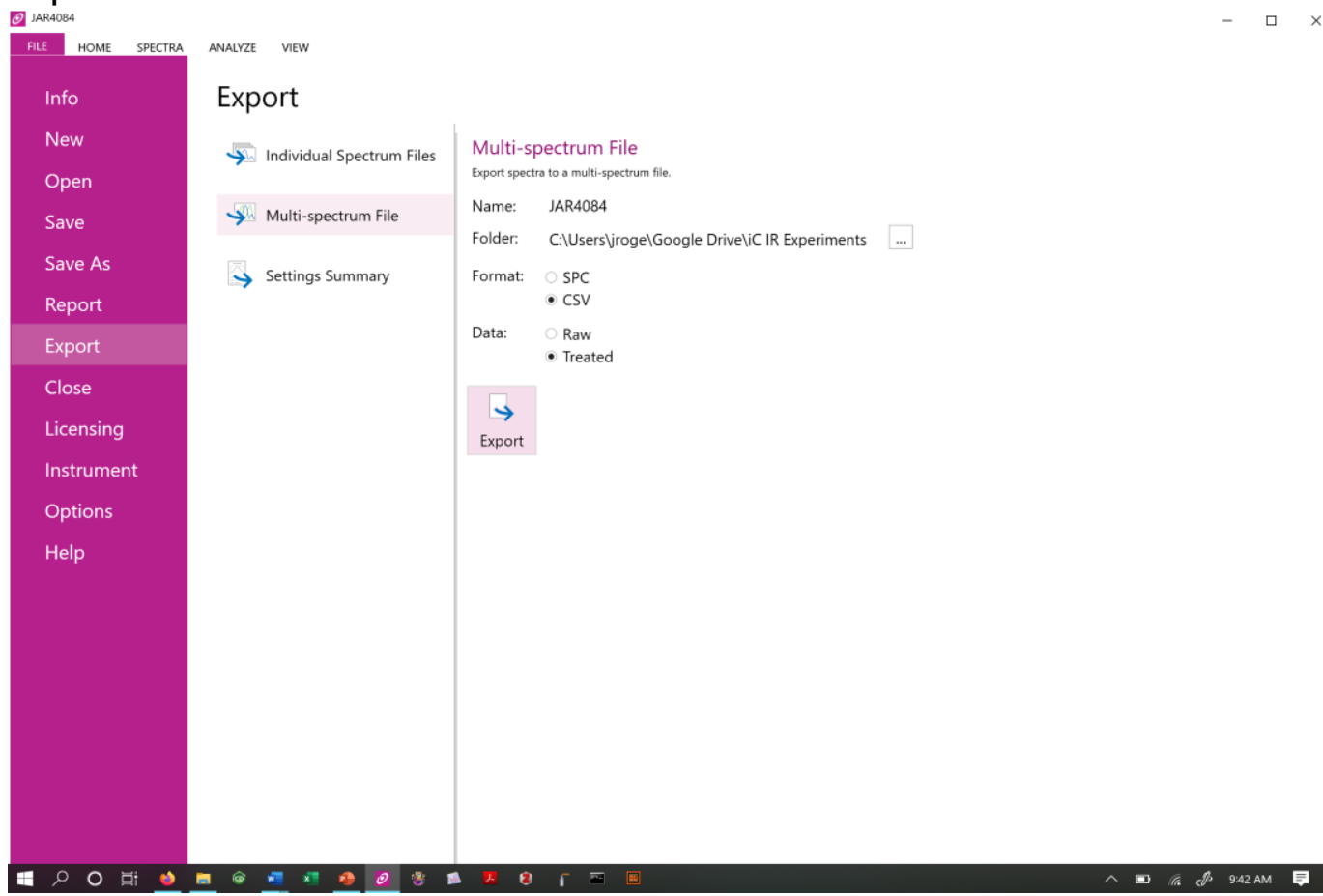

i. A new CSV file will be generated and can be opened in excel for further analysis. 


\section{IGOR Tutorial}

\section{Igor procedure file:}

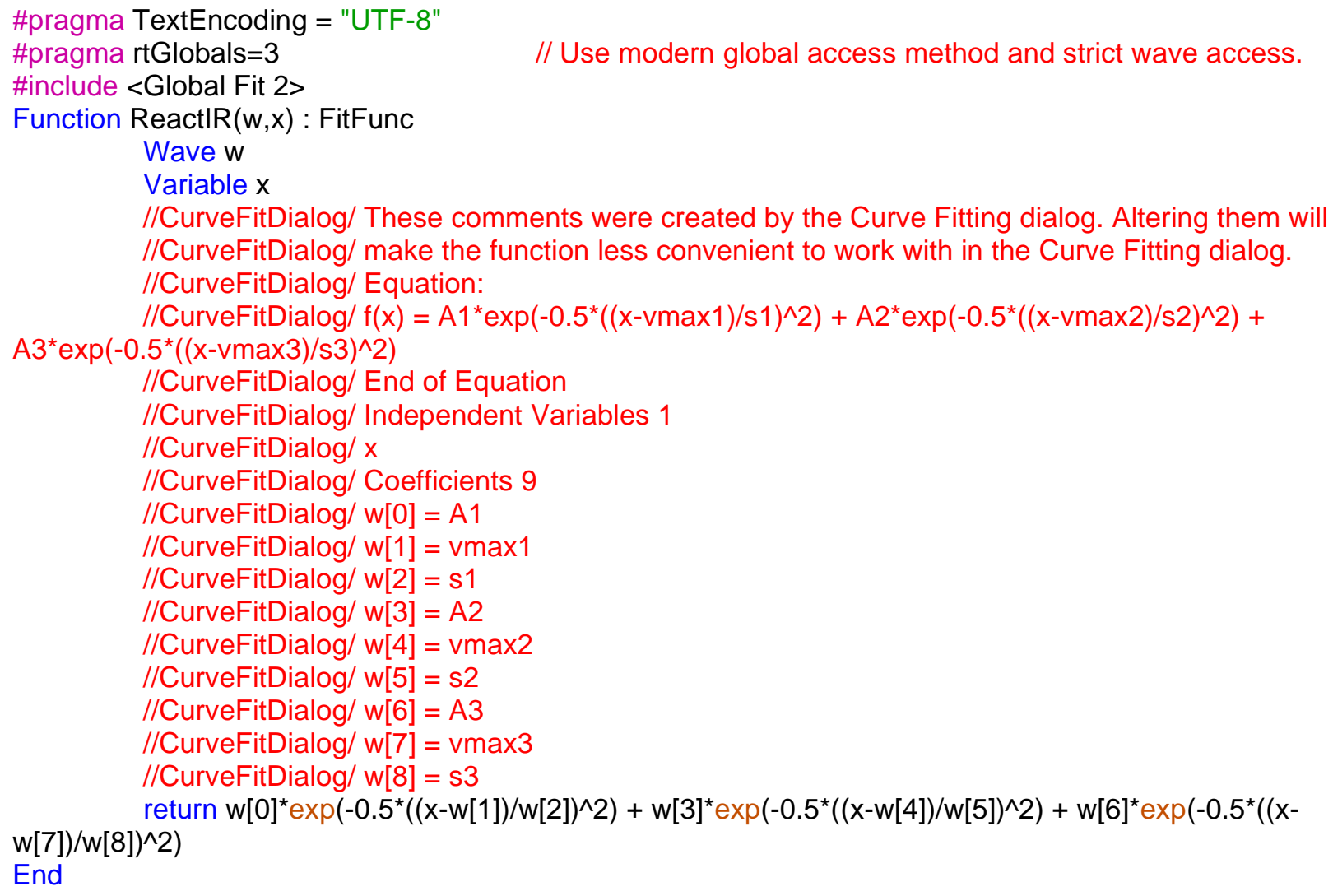


function Newmatrix()

variable i, j

wave Coef

make/o/n=(3,167) Areawave

// I made a new matrix for your two signals (two rows) with the same number of points.

$\mathrm{j}=0 \quad / / \mathrm{l}$ am starting at the matrix index value of 0 of the Coef wave

Do $\quad / /$ loop of a program

Areawave[0][j]=(Coef[0][j] ${ }^{*}$ Coef[2][j])/(1/(sqrt(2*i)))

// I am inputting the equation, I am saying that the calculated area should go into the first row and "jth" $\mathrm{j}+=1 \quad / / \mathrm{l}$ am telling the program to go to the next column in the row

while $(j<167) / /$ this is when I want the loop to stop - when the value of $j$ hits the end of the row

$\mathrm{j}=0 / / \mathrm{I}$ am resetting the index number back to the first box of the first row

Do //repeating for the second signal data set

Areawave[1][j] $=($ Coef[3][j]*Coef[5][j])/(1/(sqrt(2* pi)))

$\mathrm{j}+=1$

while $(j<167)$

$\mathrm{j}=0$

Do //repeating for the second signal data set

Areawave[2][j]=(Coef[6][i] ${ }^{\star}$ Coef[8][i])/(1/(sqrt(2*i) $\left.)\right)$

while $(j<167)$

j+=1

edit areawave

display areawave[0][*]

appendtograph areawave[1][*]

appendtograph areawave[2][*]

ModifyGraph $\mathrm{rgb}$ (Areawave) $=(1,4,52428)$

ModifyGraph rgb(Areawave\#2) $=(0,0,0)$

Label left "I\F'Arial'IZZ14 Area";DelayUpdate

Label bottom "\\F'Arial'|\Z14 Time Point";DelayUpdate

ModifyGraph fsize $=12$

end

Function makeintersect()

variable i, j

wave Coef

make/o/n=(3,167) intersect

$\mathrm{j}=0$

Do

intersect[0][j] $=((\operatorname{Coef}[1][j] /$ Coef[2][j] $)+(\operatorname{Coef}[4][j] / \operatorname{Coef}[5][j])) /((1 / \operatorname{Coef}[2][j])+(1 / \operatorname{Coef[5][j]}))$

$\mathrm{j}+=1$

$j=0$

while $(j<167)$

Do

intersect[1][j] $=((\operatorname{Coef}[4][j] / \operatorname{Coef}[5][j])+(\operatorname{Coef}[7][j] / \operatorname{Coef}[8][j])) /((1 / \operatorname{Coef}[5][j])+(1 / \operatorname{Coef}[8][j]))$

$\mathrm{j}+=1$

$\mathrm{j}=0$

while $(j<167)$

Do

intersect[2][j] $=((\operatorname{Coef}[1][j] /$ Coef[2][j] $)+(\operatorname{Coef}[7][j] / \operatorname{Coef}[8][j])) /((1 / \operatorname{Coef}[2][j])+(1 / \operatorname{Coef}[8][j]))$

$j+=1$

edit intersect

while $(j<167)$

end 


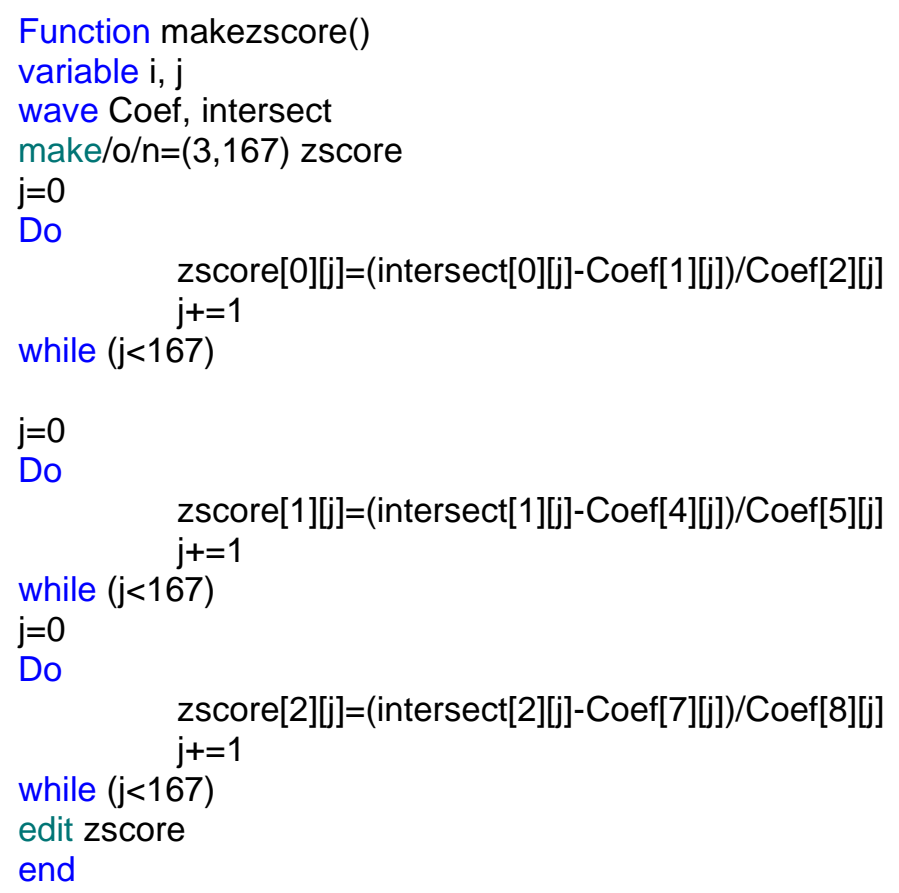

1) To begin IGOR fitting open the procedure file by following the drop down under open file tab.

2) Data should be in the following format

3) Using the command bar type "edit"

\begin{tabular}{|c|l|l|l|}
\hline Signal & \multicolumn{2}{|l|}{ Time points } & \\
\hline & & & \\
\hline & & & \\
\hline
\end{tabular}

4) Copy the data table into the new table. (Rows and Columns start with 0 )

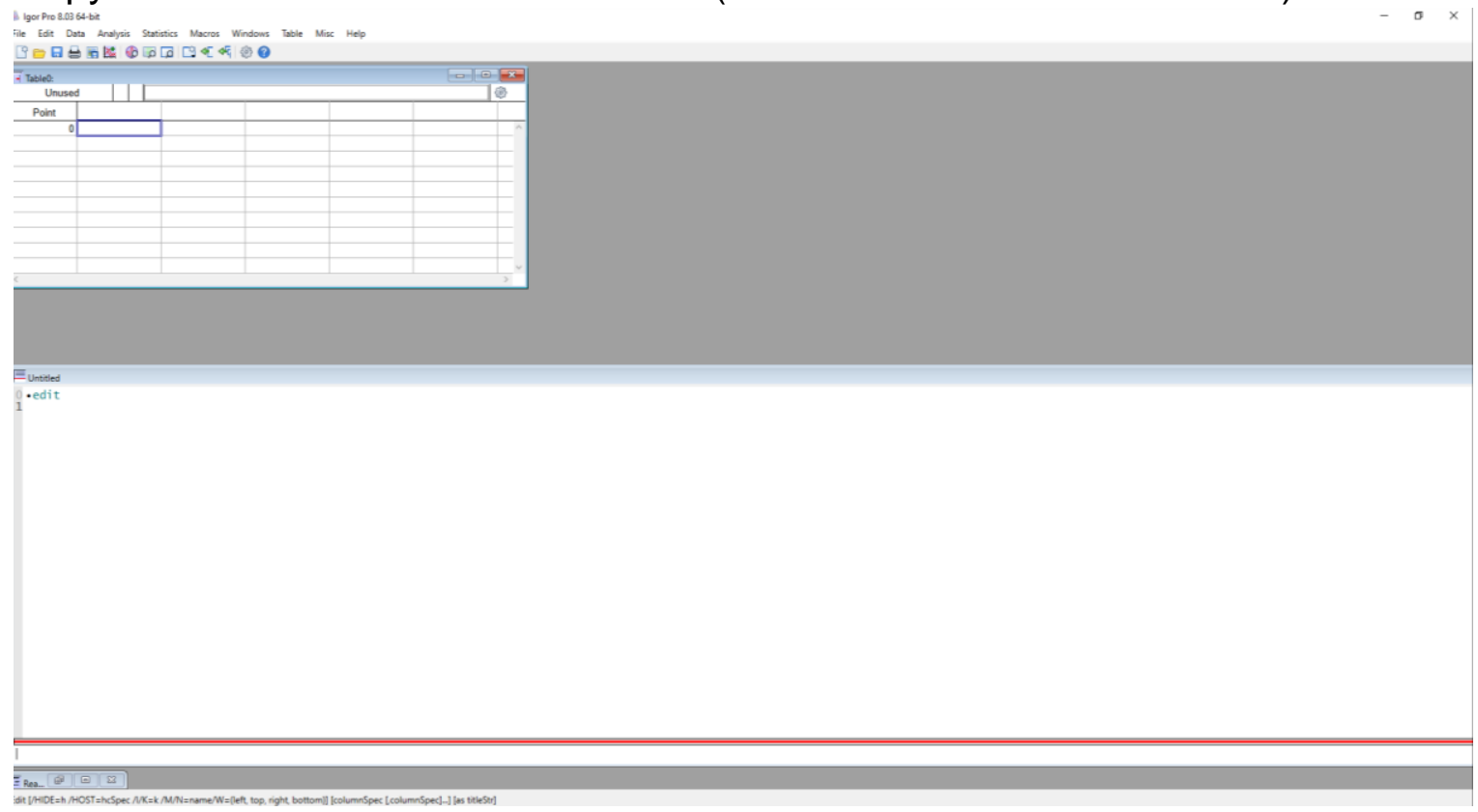


5) Under the windows tab select "new graph", then select waveX vs wave0 to graph.

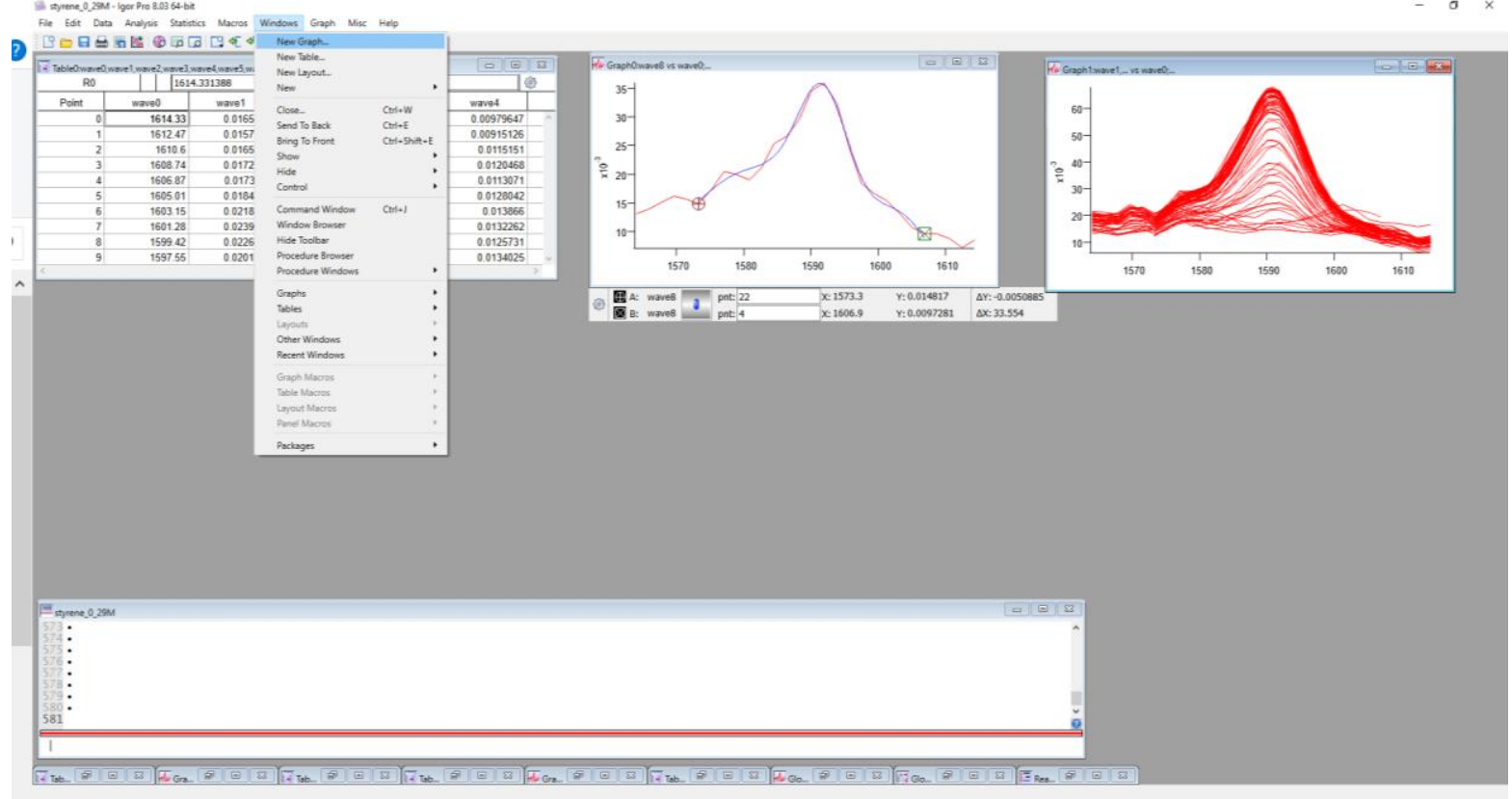

6) Under the windows tab select "new graph", then select all waves vs wave0 to graph. 7) Under the analysis tab select "curve fit", then select your function, $y$ data, $x$ data, and whether the data set needs to be truncated. "ctrl + i" allows you to select cursors. Input coefficient values and select "graph now," then "do it."

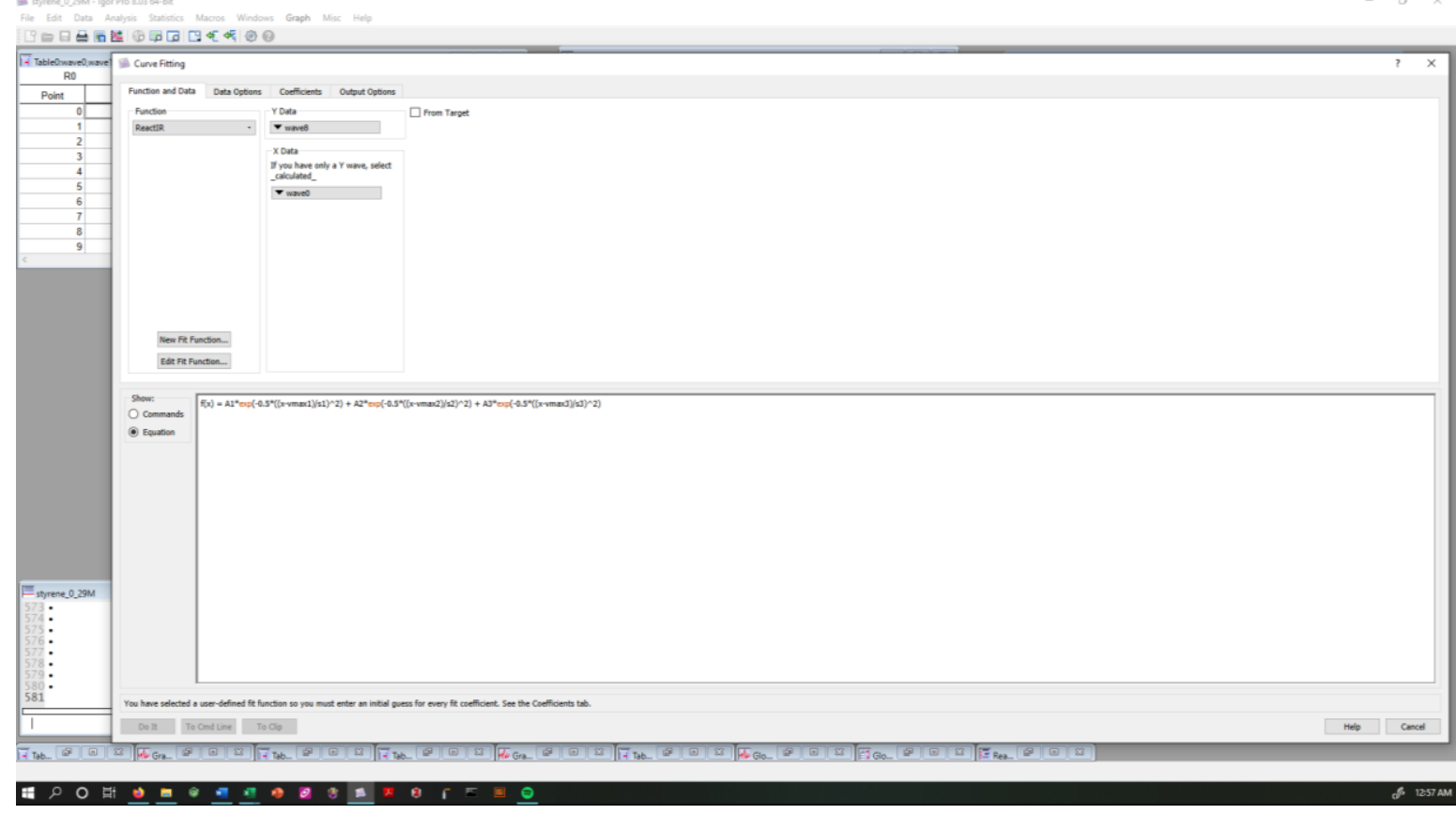




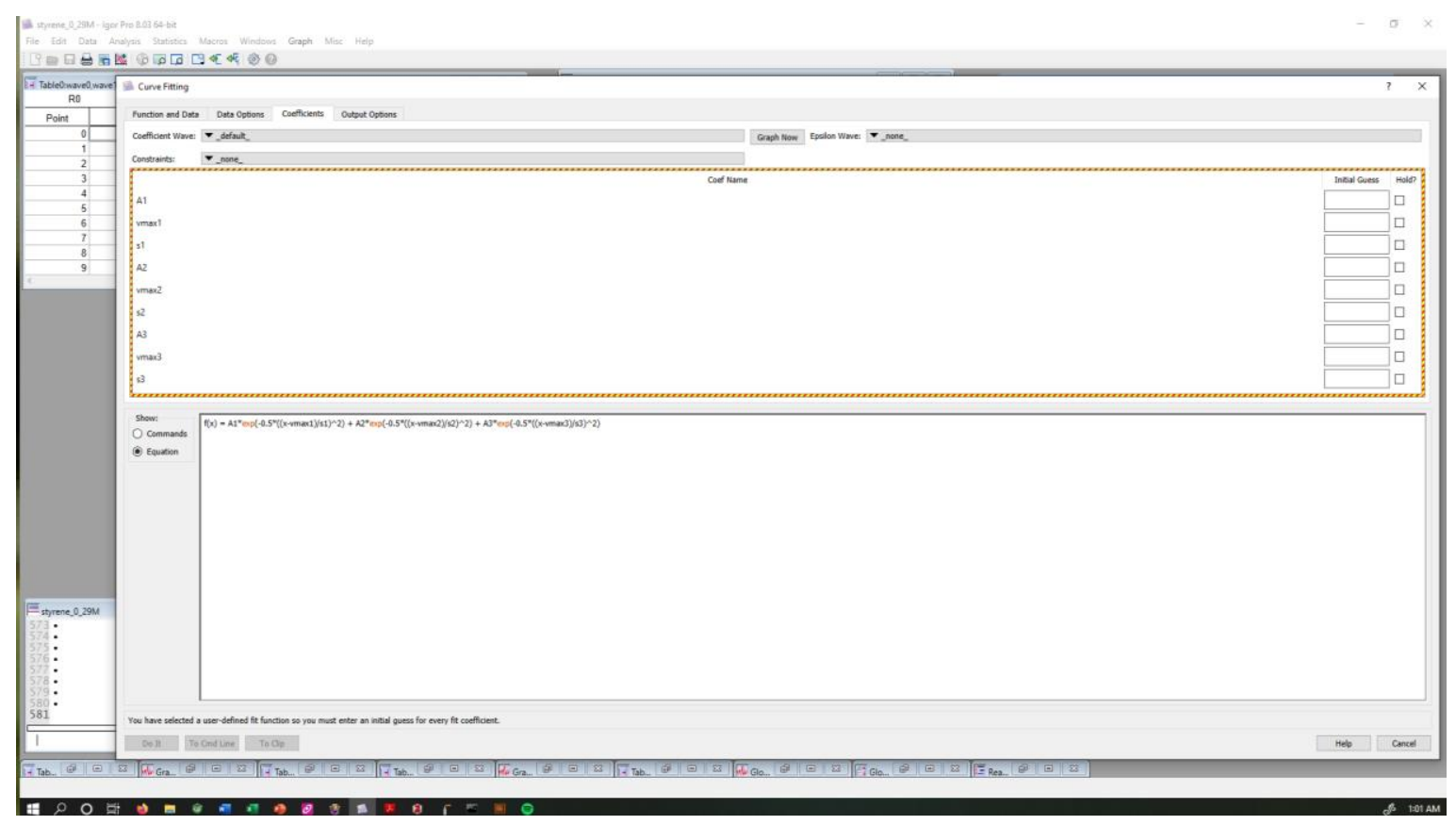

8) In the command bar type "make/n=(\#of rows) mask" then "mask ["cursorA","cursorB"]=1"

9) Under the analysis tab select the "global fit"

10)_Add data set then highlight the "function" tab and select the desired function. Then select each variable and link them.

11)Select masking and highlight the dropdown and select "mask"

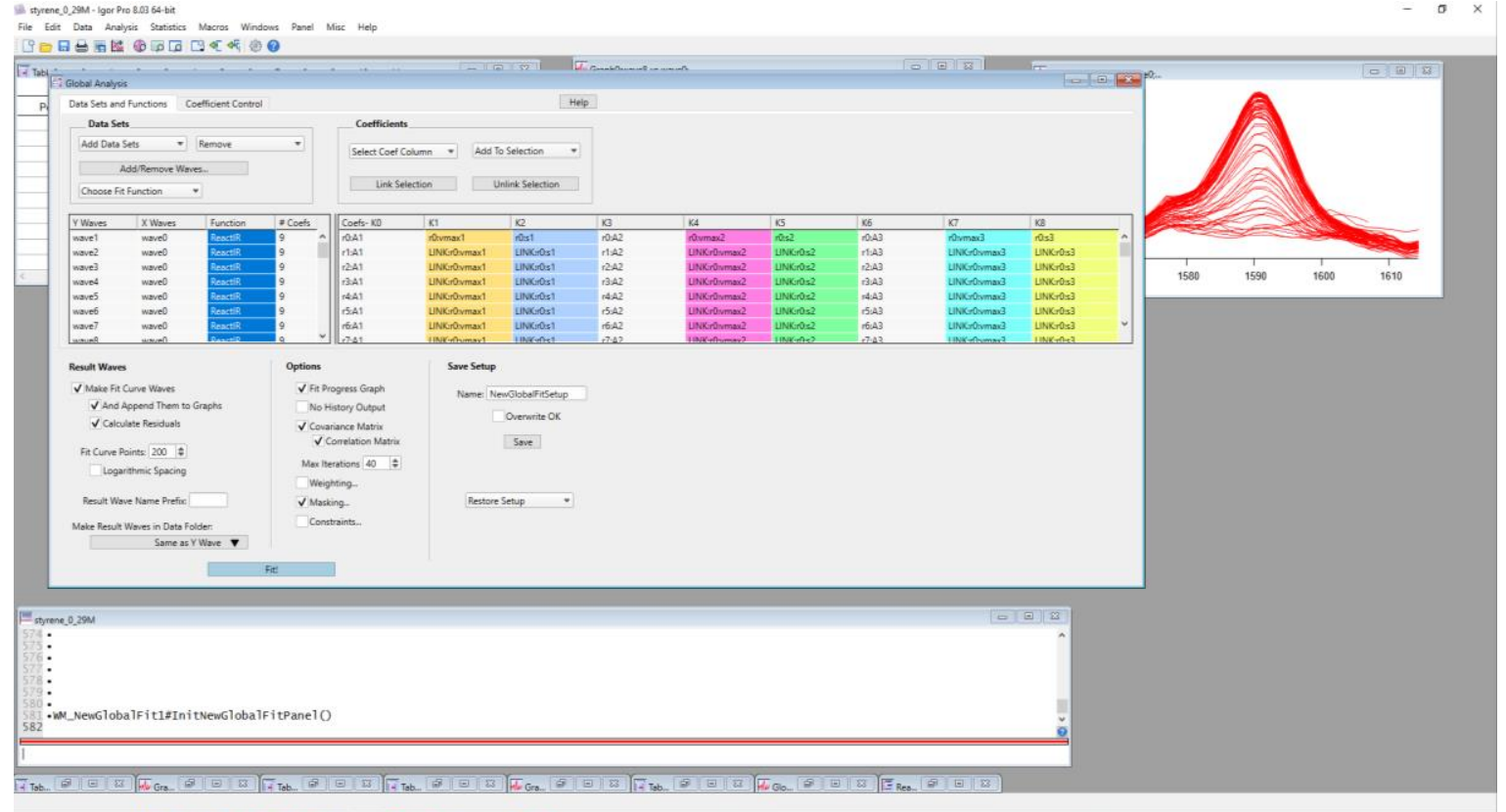


12) Under Coefficient control select the wave with respect to the curve fit wave from step 7. Check the wave number (vmax) and width (s) for each gaussian curve. Then under the "datasets function" tab unlink all of the columns with regard to absorbance, and select "fit".

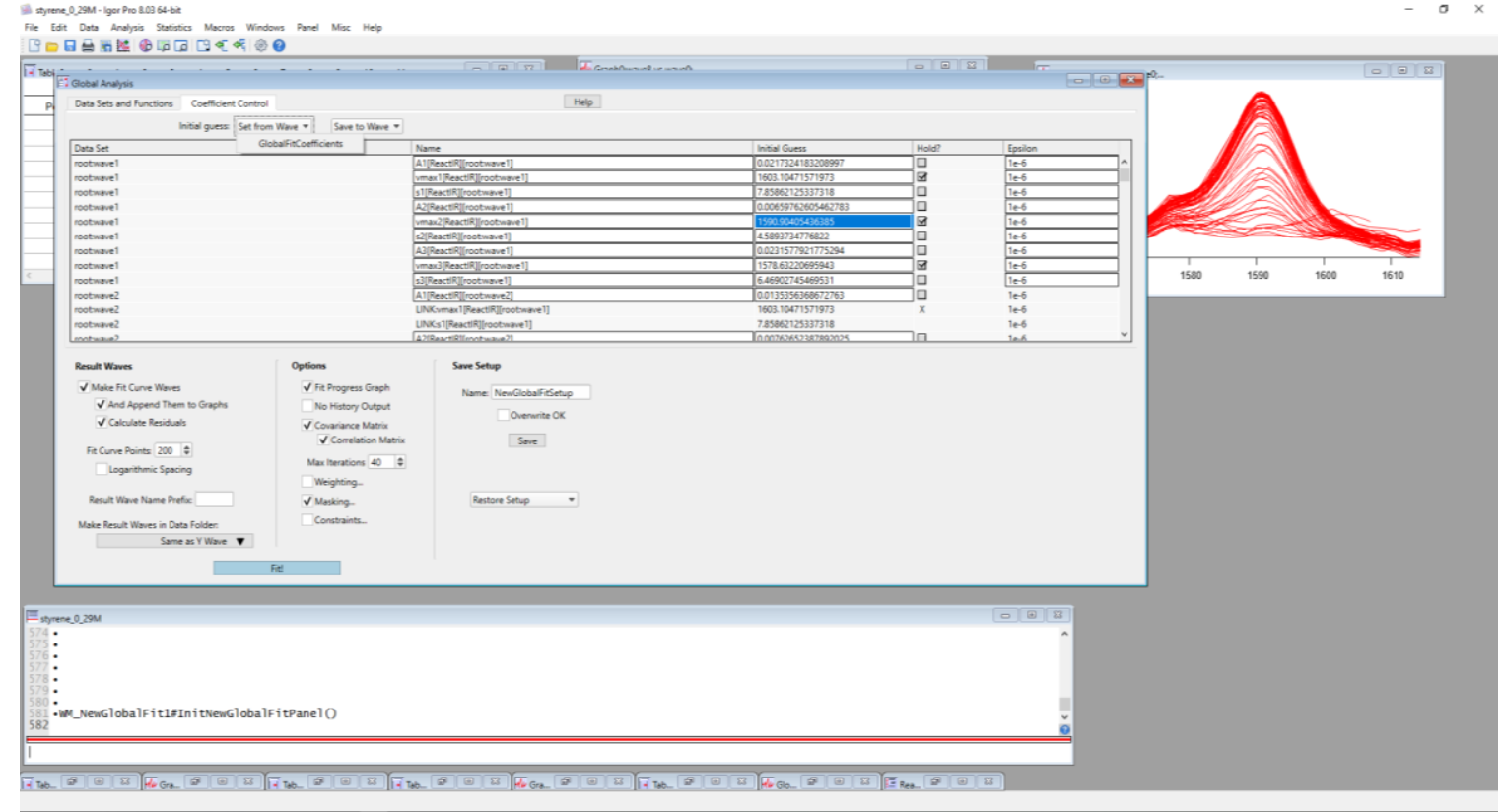

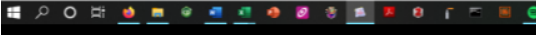

13) Type the following "makematrix(); newmatrix(); makeintersect(); makezscore()"

a. This opens new tables and graphs.

14) Save the zscore and area table as a .csv or .txt file.

15)Calculate any other information needed in Excel. 


\section{COPASI Tutorial}

1) Save timecourse data as a txt file with time in column $A$.

2) Open COPASI and open the Model Drop Down

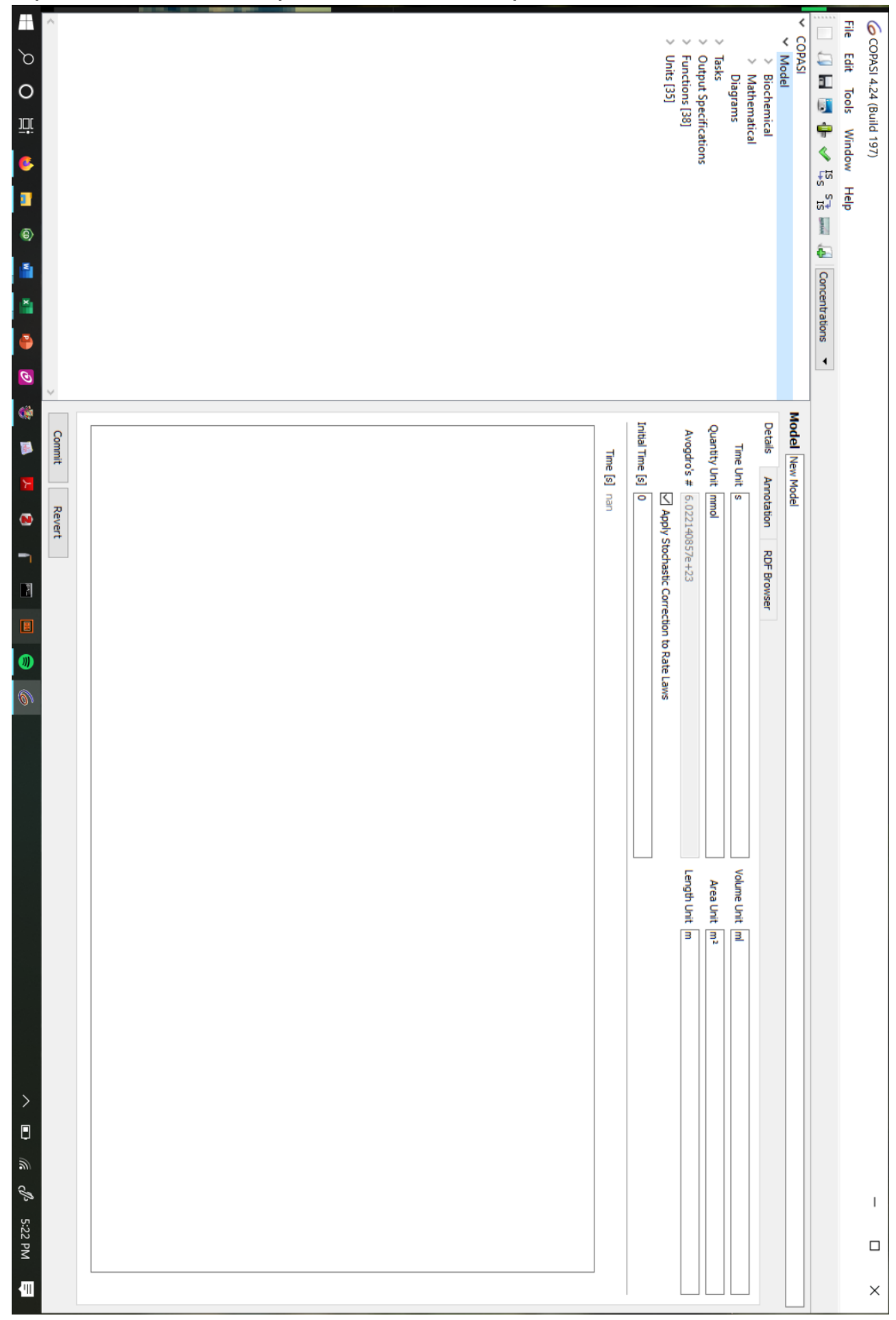


3) Under compartment name your "vessel" and identify the unit size. (reaction volume)

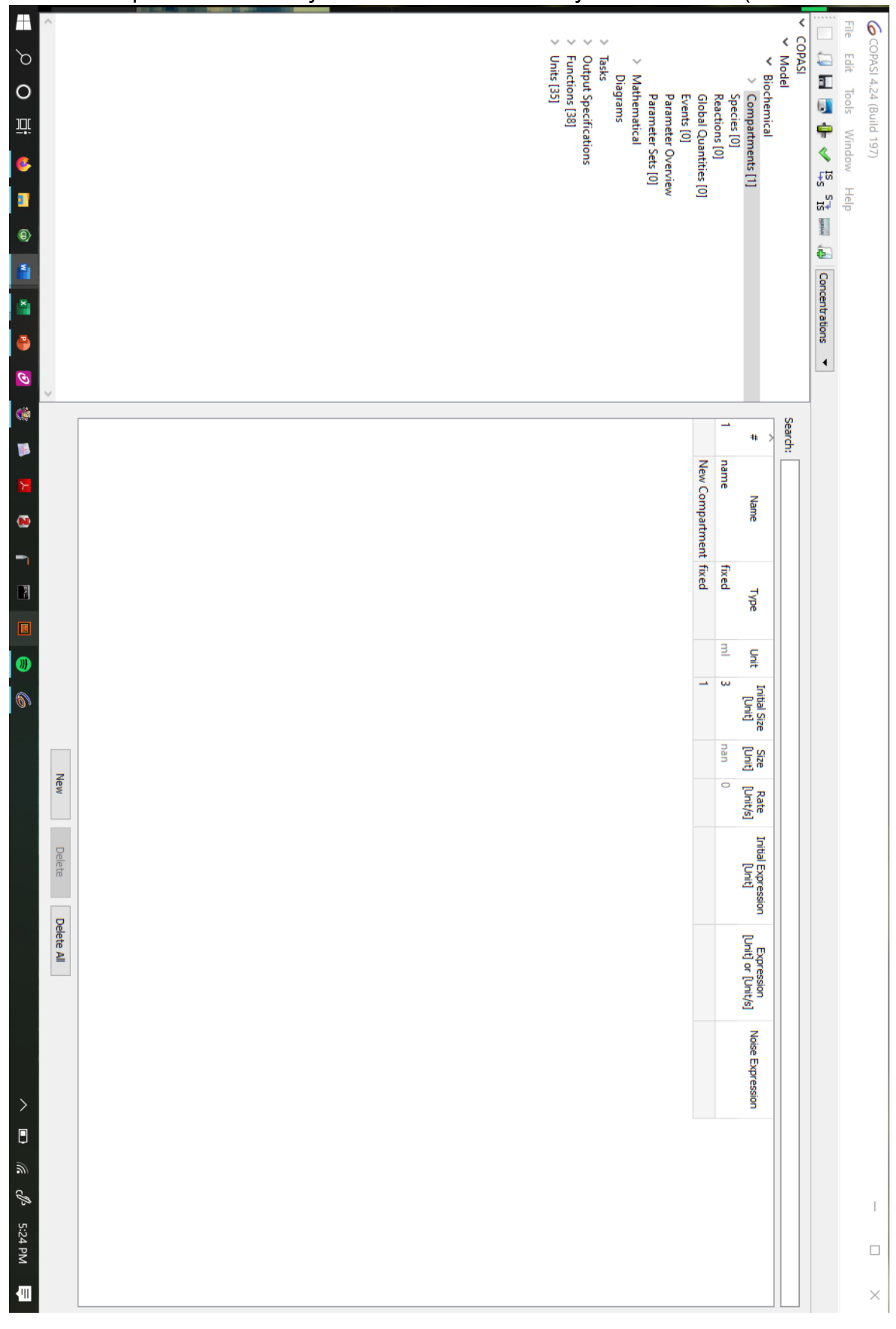


4) Under reactions

a. Type the name for reactions (numbers are helpful here)

b. Type in the reaction " $A+B=C$ " for reversible and " $A+B-C$ " for irreversible

c. You must have spaces.

d. $(a \neq A)$

e. Repeat as necessary

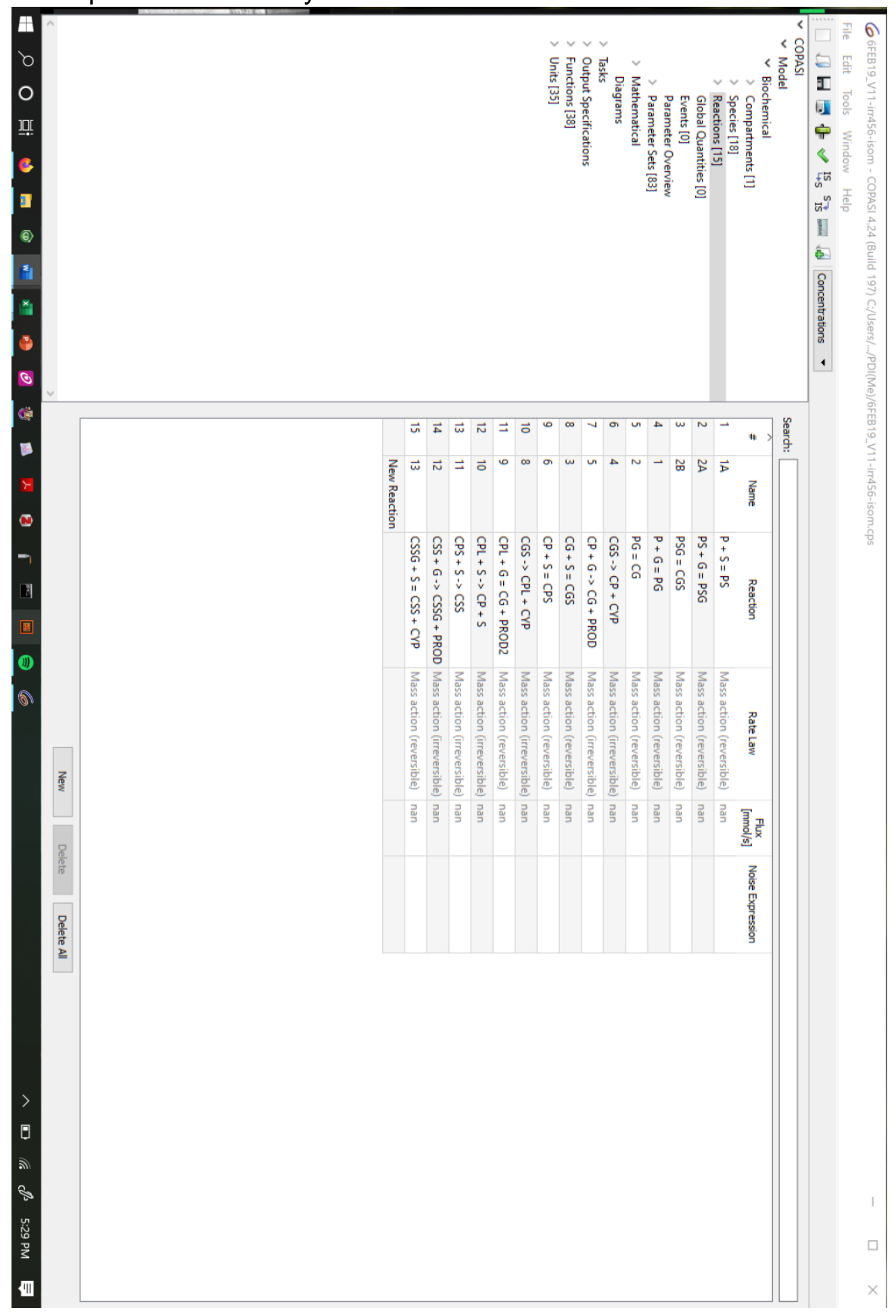


5) Under the "Species" tab. Insert the initial concentrations of your reaction mixture for each defined intermediate.

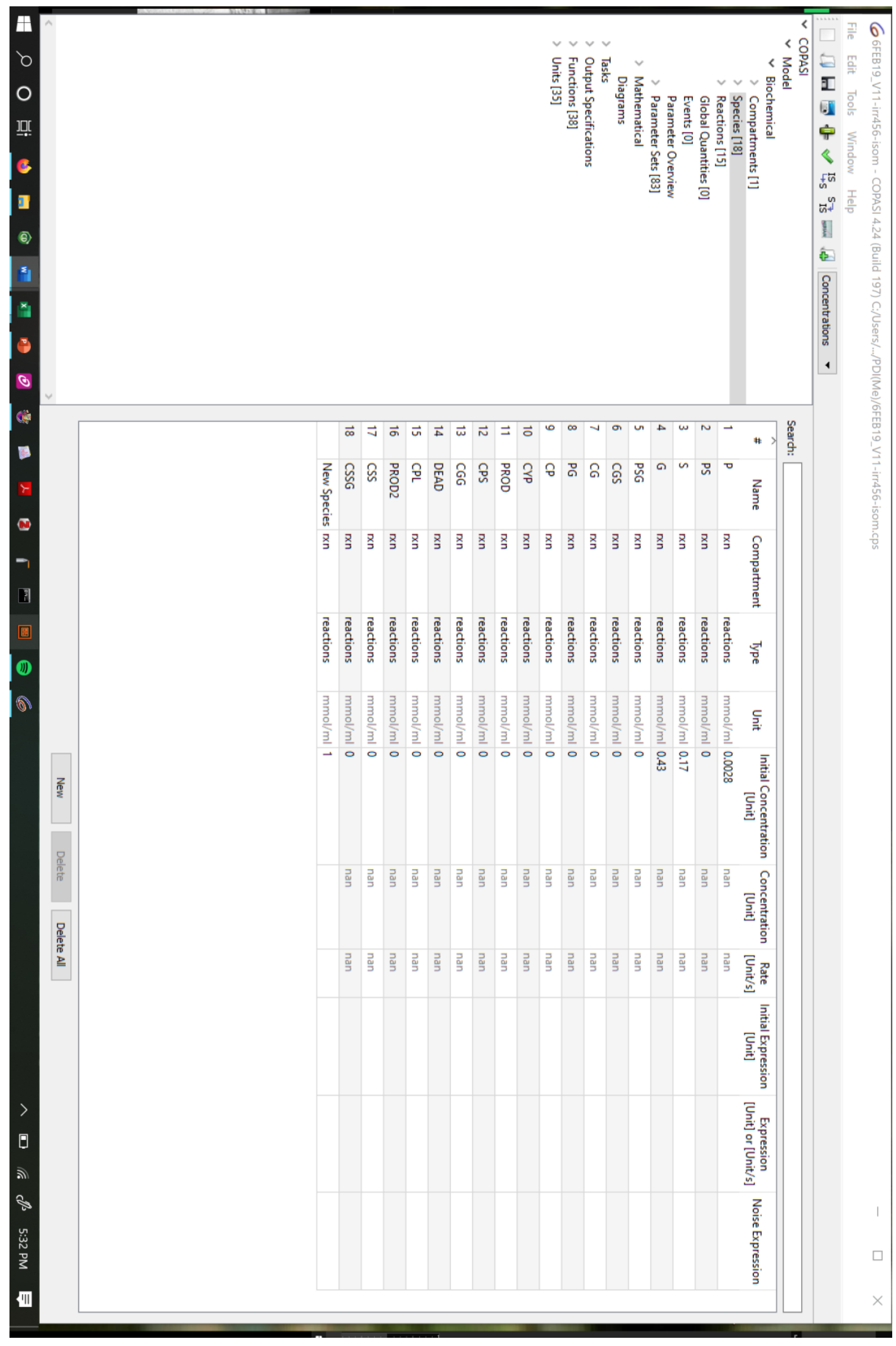

$-\mathrm{T} 25-$ 
6) Save file \{program can be glitchy and close and all data will be lost

7) Under "tasks" select "Parameter Estimation"

a. Select Experimental Data in top right corner.

b. Input data by selecting the green "+"

c. For each name the experiment select "Time Course"

d. Mean Square weight method

e. In the second row change type to dependent by double clicking the box that says "ignored"

f. Select the COPASI symbol and open the "Transient Concentration" drop down menu

g. Select the proper variable. Select "Ok" and repeat as necessary.

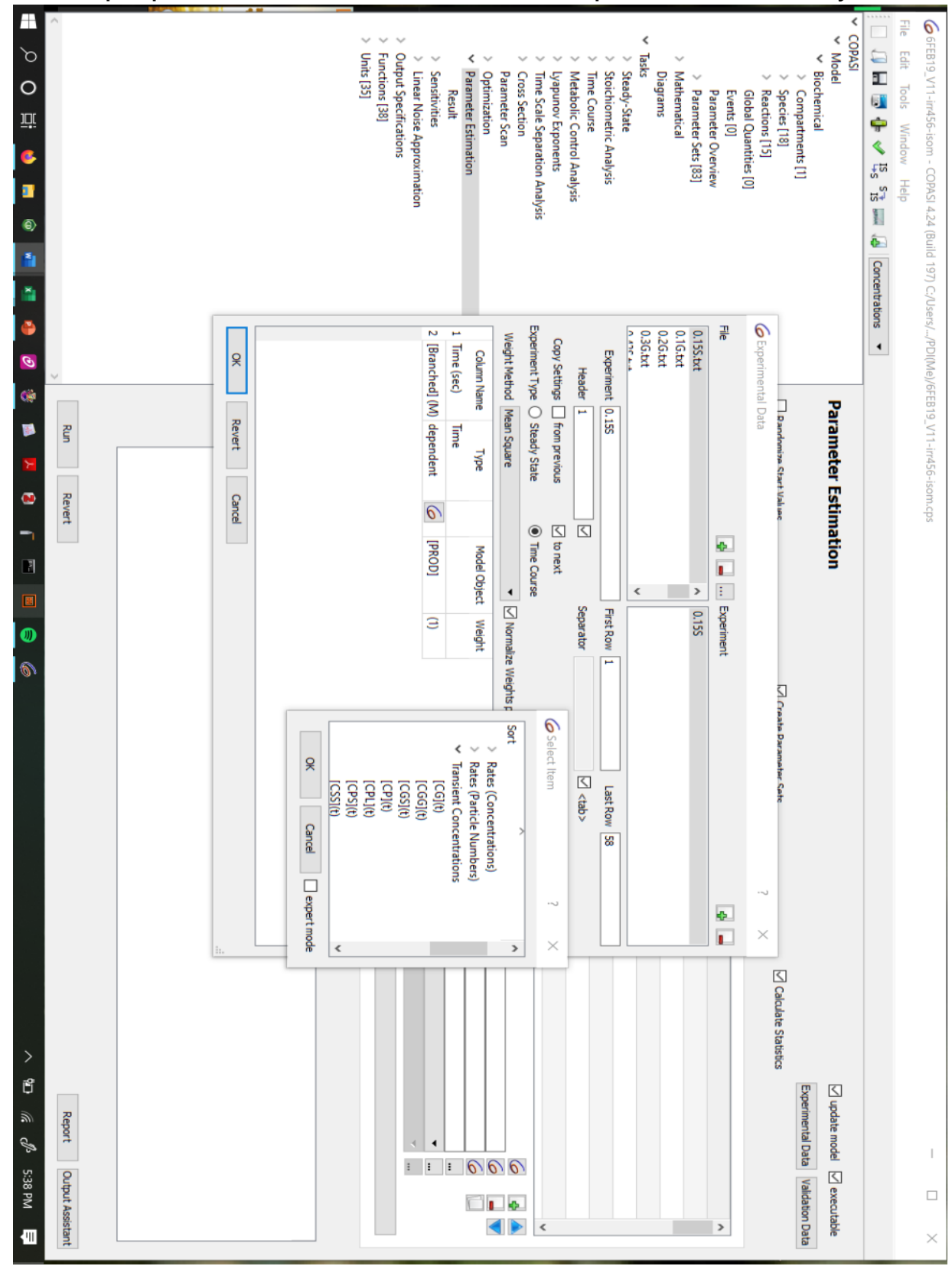


8) The Data window will close upon finalizing data entry.

9) Under object select the COPASI symbol

a. Under the new window select "Reactions"

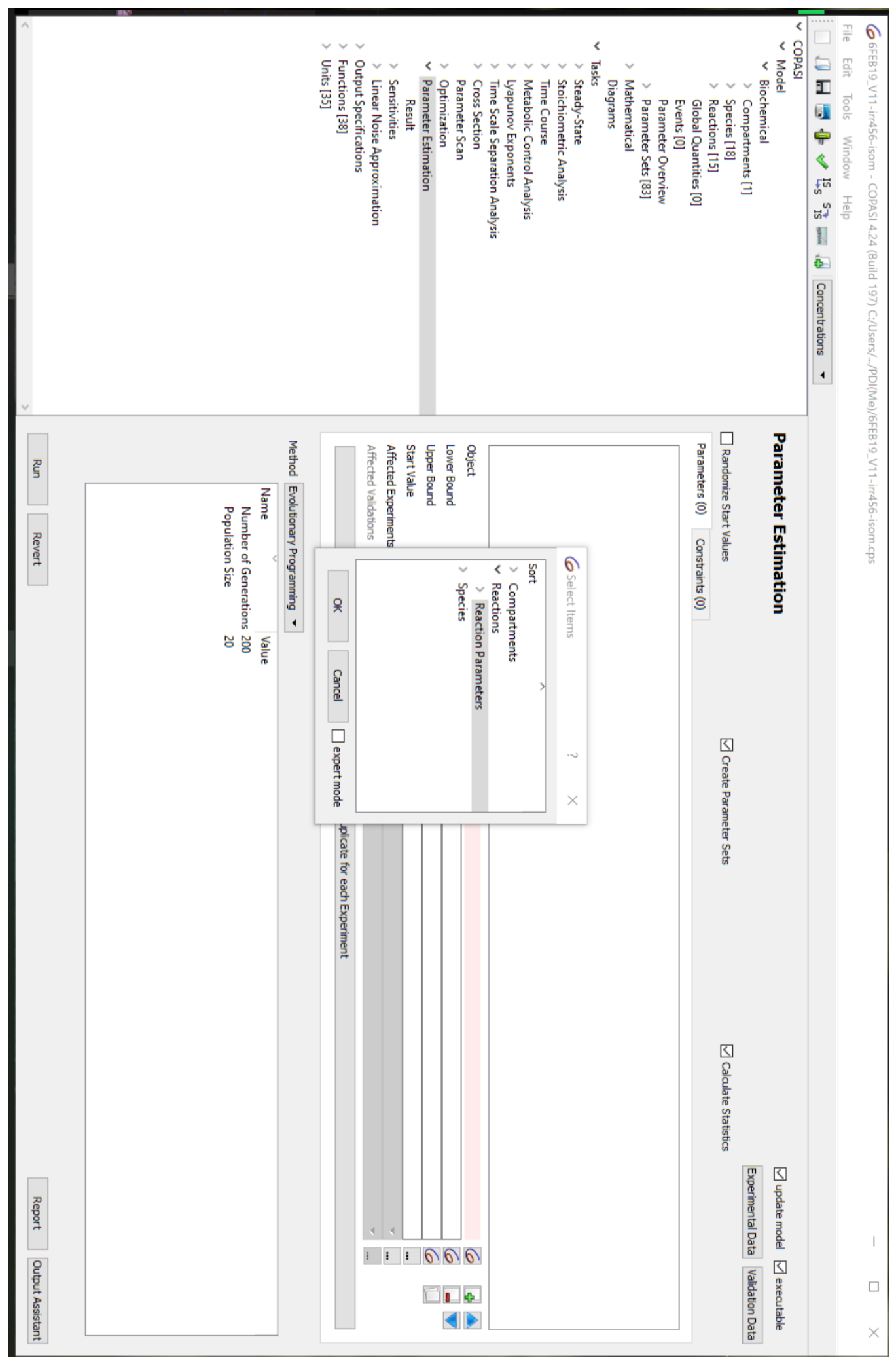


10) This inserts all reaction constant parameters.

a. You may choose the "lower" and "upper" bounds by highlighting all fields and typing in a new number.

b. Check the box for "Randomize Start Values," "Create Parameter Sets," "Calculate Statistics," "Update Model," and "executable"

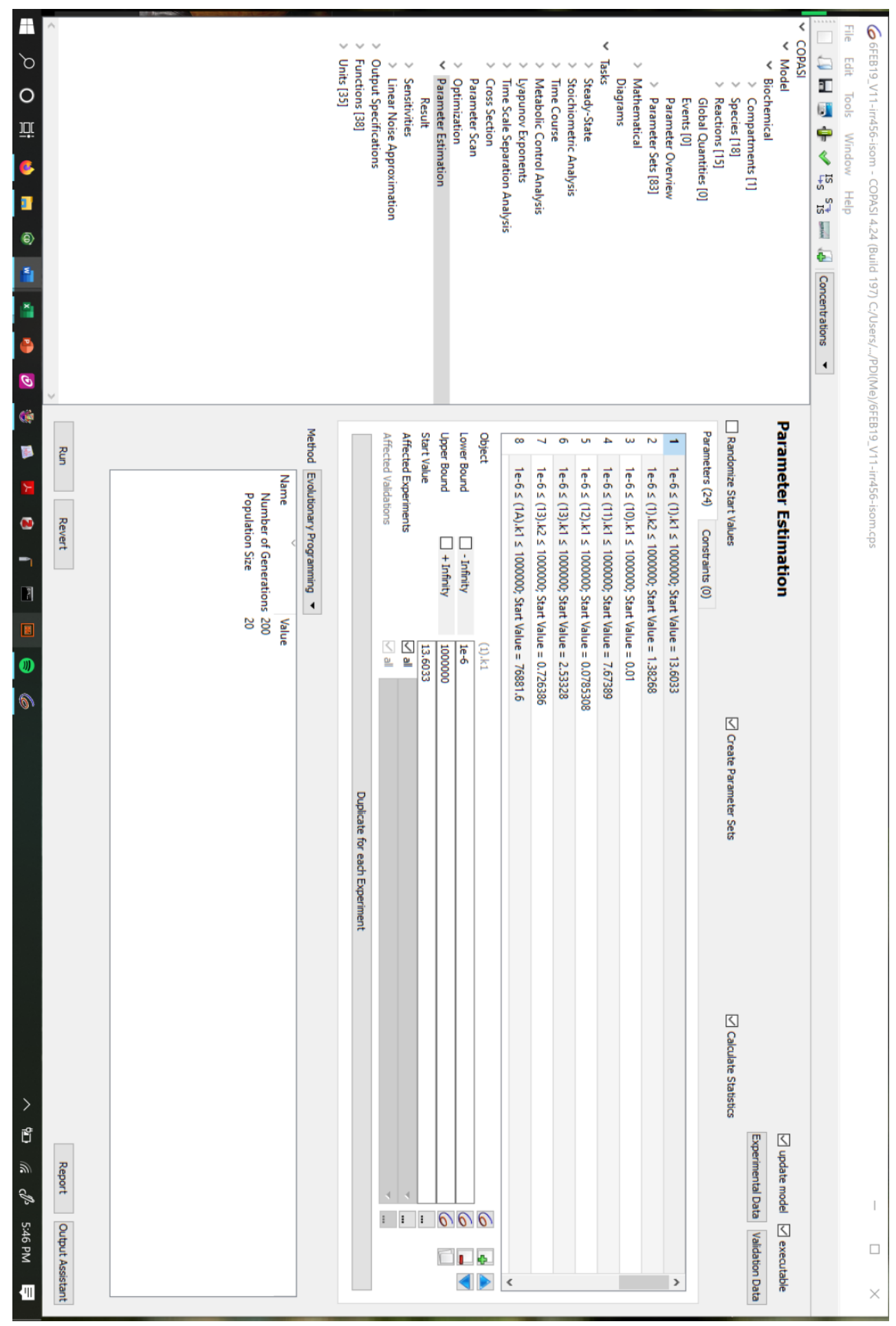


11)Select the Green "+" and using the blue toggle arrows move this new item line to the top.

a. Select the COPASI symbol and then "species"

b. Under Initial Concentrations select the starting variable.

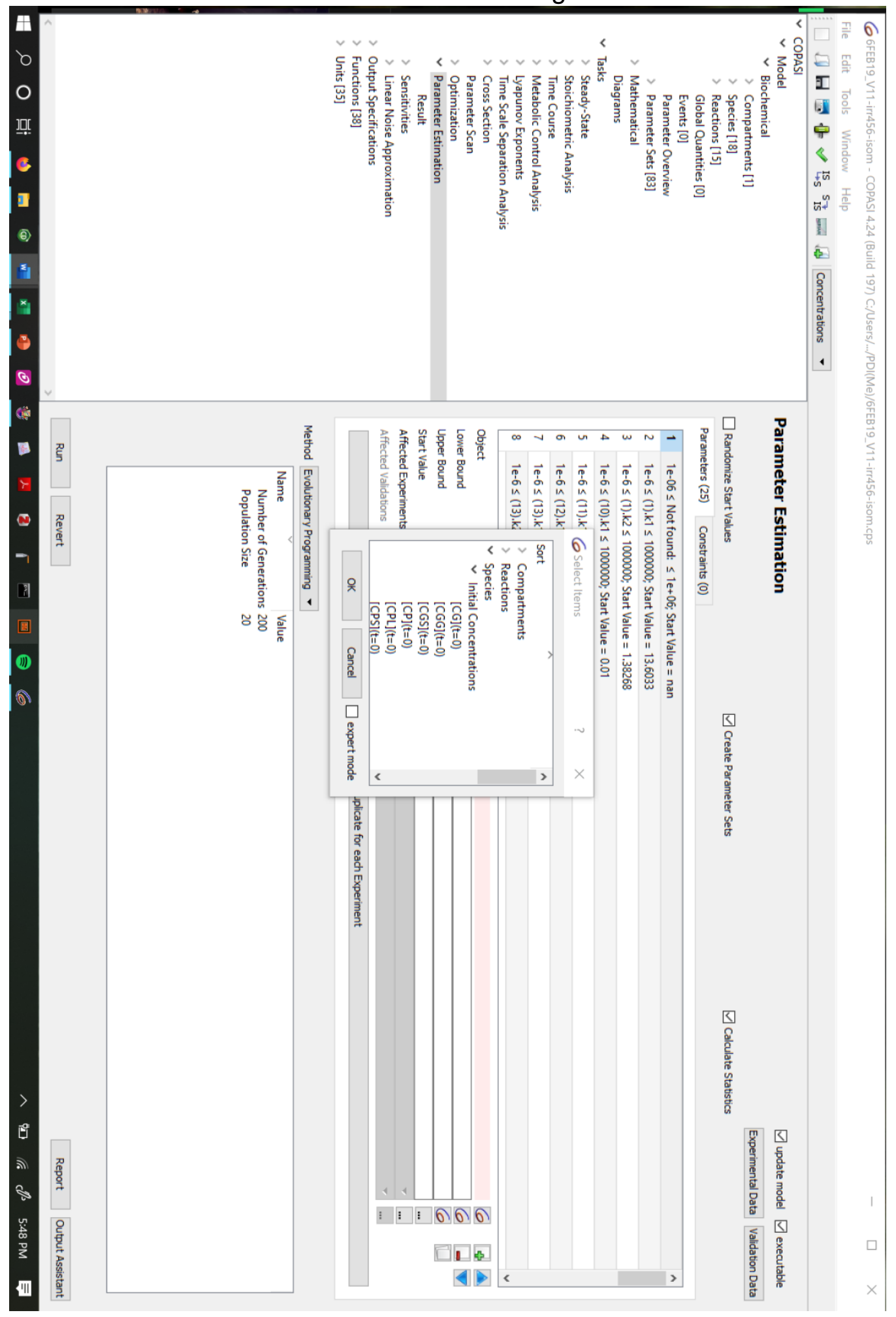


12) Change the affected Experiments by unchecking the "all" box, then select the experiments you want to use.

13) Repeat this for all initial variables that change concentration over time.

14)Once initial set up is complete ensure you are using the "Evolutionary Programming" method.

15) Select Reports and choose a filename.

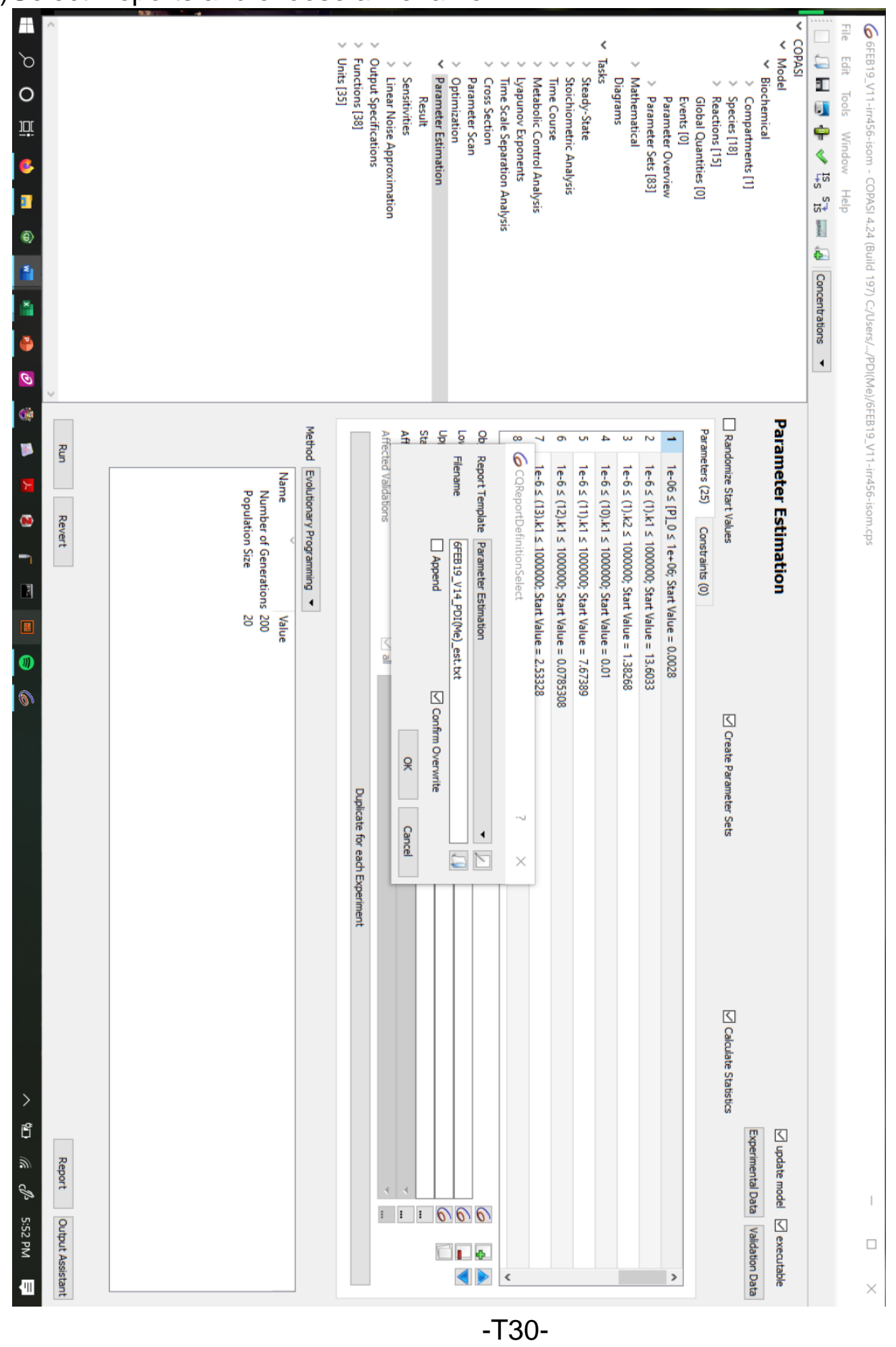


16)Select "RUN"

17) To generate a plot and simulation reports go to "Output Specifications"

a. For reports select "Report Templates"

i. Select "New" then double click the new generated report

ii. Name the type and select task as "Time-Course"

iii. Select "Item" then "Species" and "Transient Concentrations"

iv. Ensure the generation is a table. Then select "Commit"

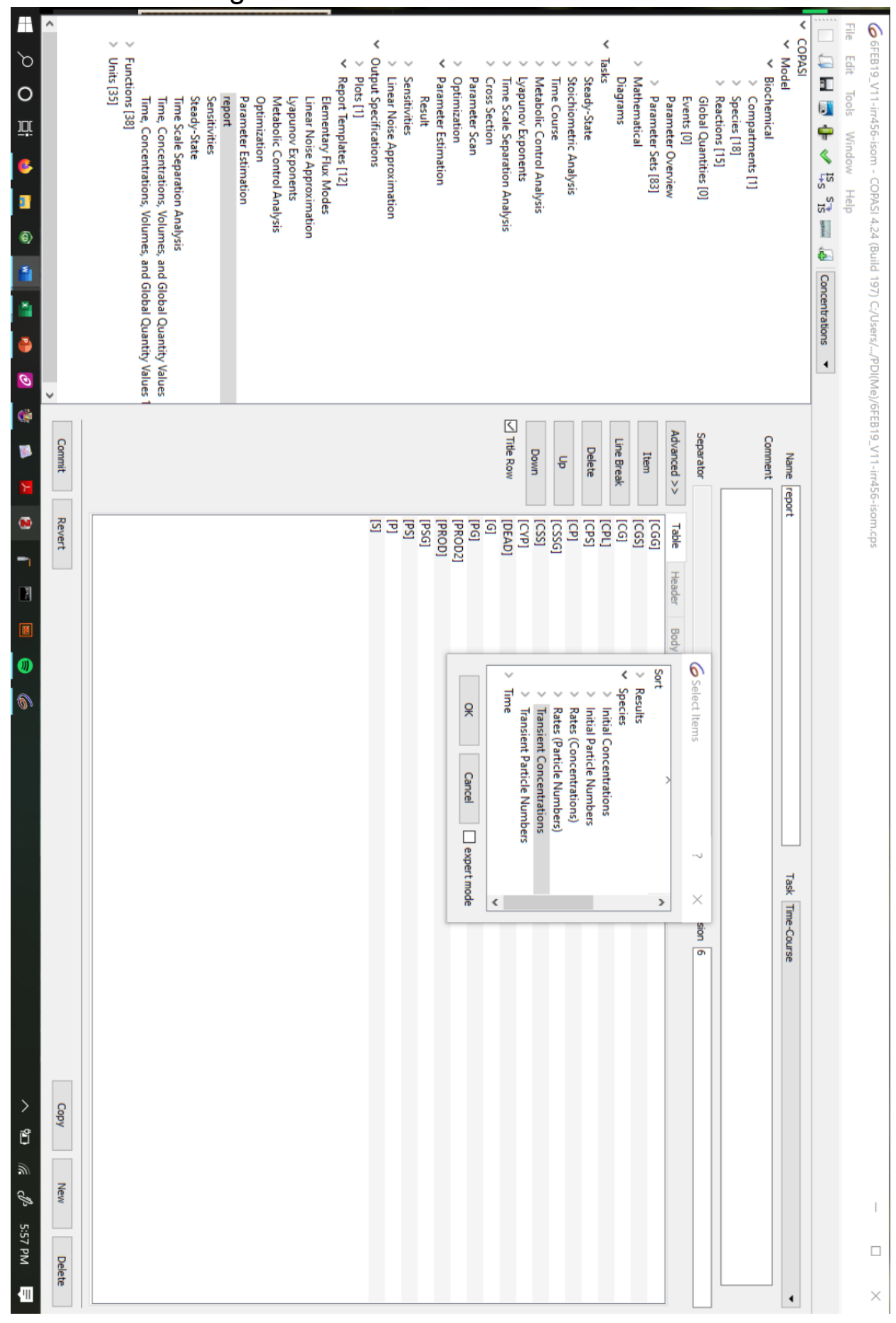


b. For plots select "Plots"

i. Select "New" then double click the new generated plot

ii. Name the type and select "New Curve"

iii. Select $x$-variable as "Model time" and y-variable as "Transient Concentrations" then specific trend you want to view.

iv. Select "Commit"

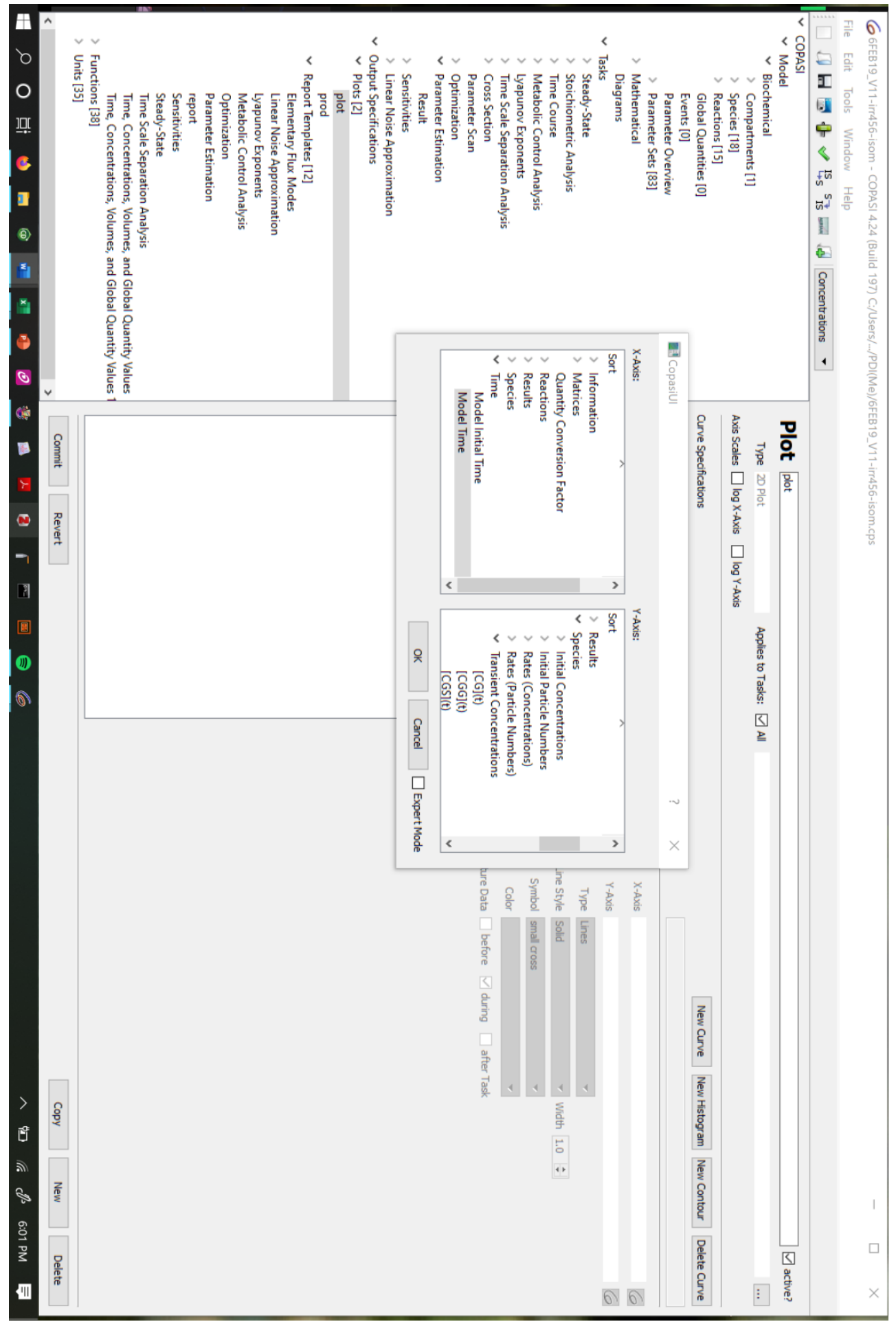


18)To run a time course first you must select your parameter set.

a. Select "Parameter Set" under the Biochemical model screen

b. Double click on the parameter you want to use

c. Select apply

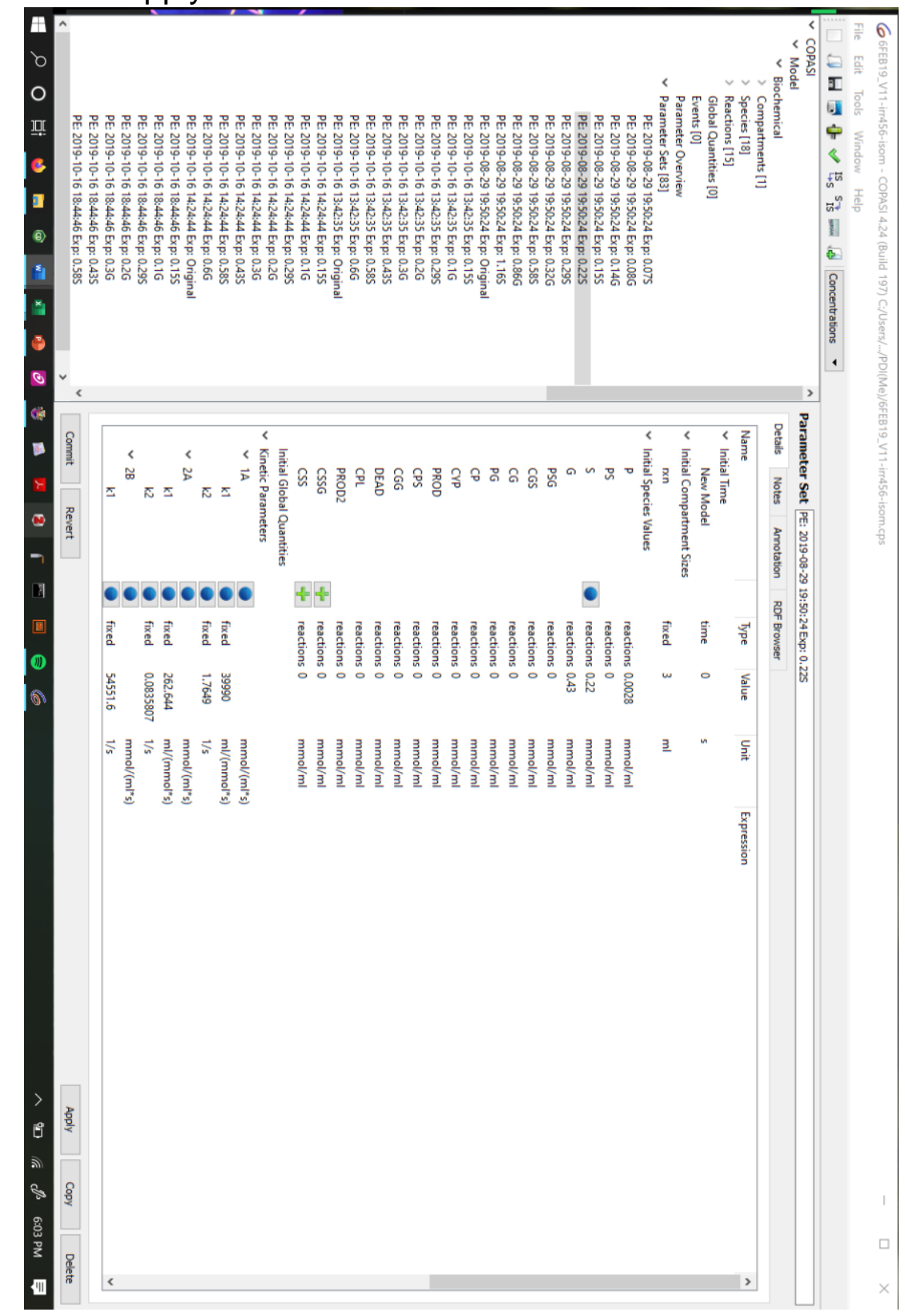

d. Select "Tasks" then "TimeCourse"

e. Insert your duration and interval size (3600s and 30s, respectively)

f. Ensure method is "Deterministic (LSODA)"

g. Select "output assistant" on the bottom right of screen.

h. Under reports select "Time, Concentrations, Volumes, and Global Values" then "Create" 


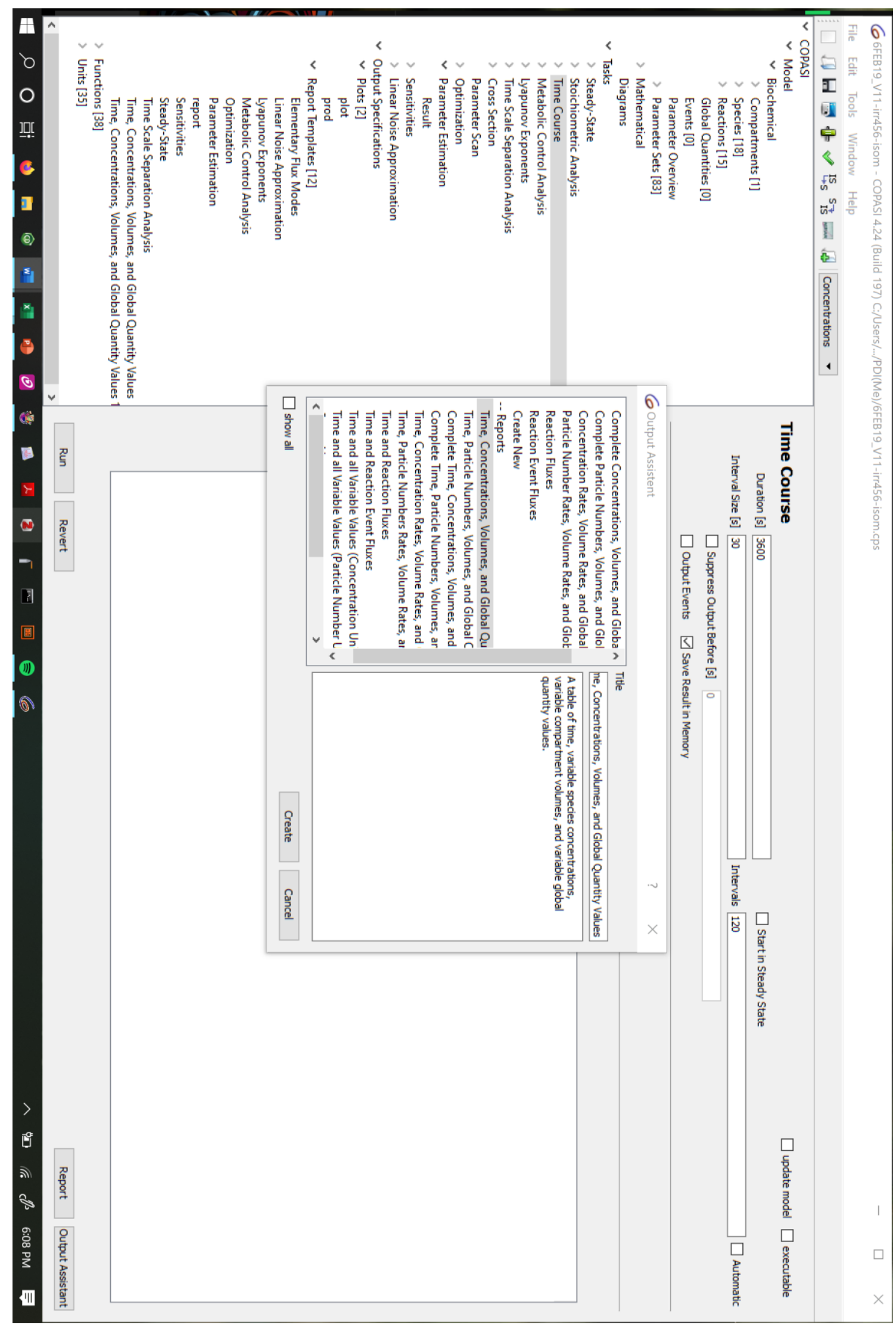


i. Select "Report"

j. Name the file and select the newly generated template from drop down

k. Select "ok" then "run"

I. Repeat this as needed for all experimental timecourses

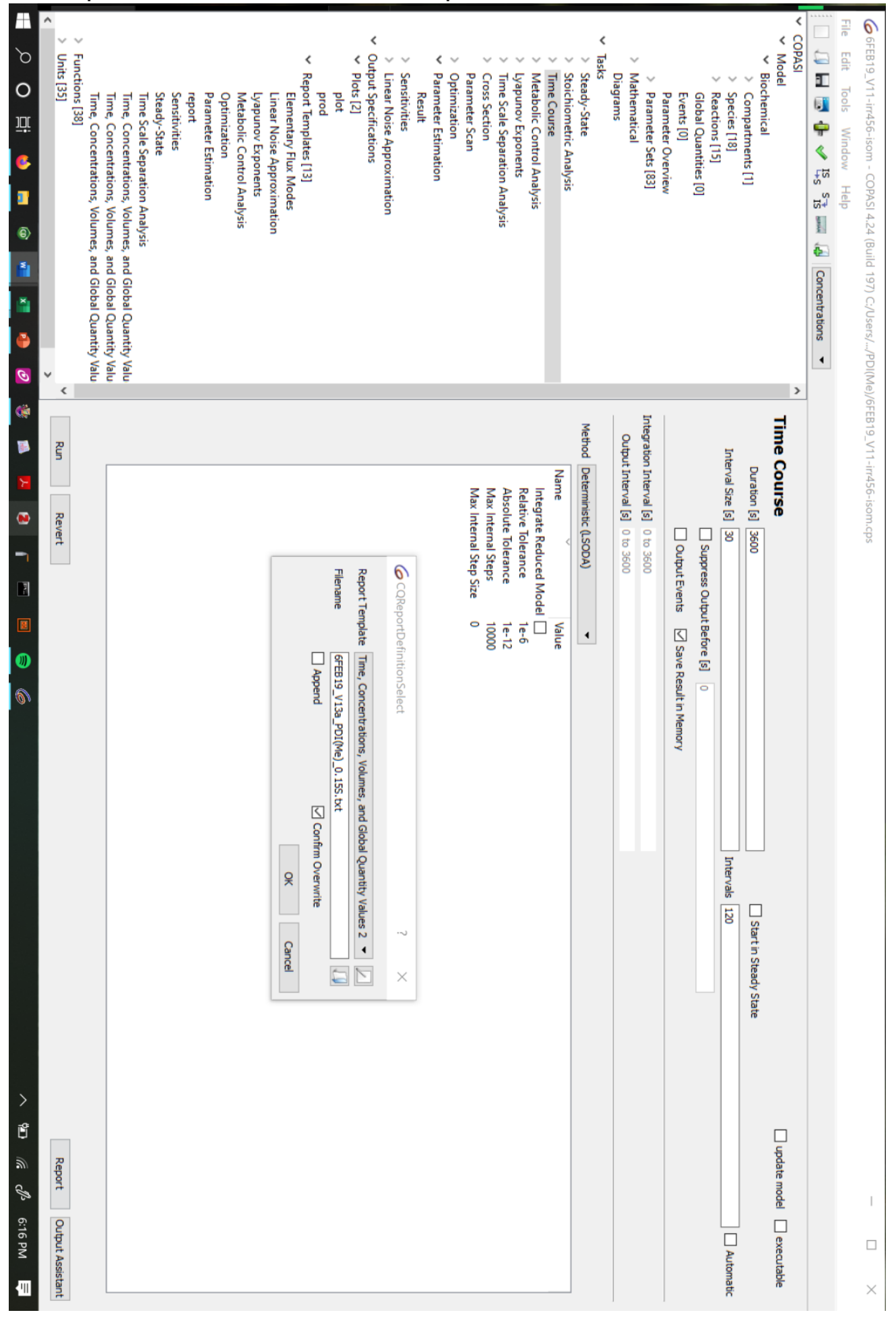




\section{References}

(1) Rogers, J. A.; Popp, B. V. Operando Infrared Spectroscopy Study of IronCatalyzed Hydromagnesiation of Styrene: Explanation of Nonlinear Catalyst and Inhibitory Substrate Dependencies. Organometallics 2019.

https://doi.org/10.1021/acs.organomet.9b00492.

(2) Bauer, E. B. Iron Catalysis: Historic Overview and Current Trends. In Iron Catalysis II; Bauer, E., Ed.; Topics in Organometallic Chemistry; Springer International Publishing: Cham, 2015; pp 1-18. https://doi.org/10.1007/3418_2015_107.

(3) Anderson, J. S.; Rittle, J.; Peters, J. C. Catalytic Conversion of Nitrogen to Ammonia by an Iron Model Complex. Nature 2013, 501 (7465), 84-87. https://doi.org/10.1038/nature12435.

(4) Jacobs, G.; Ma, W.; Gao, P.; Todic, B.; Bhatelia, T.; Bukur, D. B.; Davis, B. H. The Application of Synchrotron Methods in Characterizing Iron and Cobalt FischerTropsch Synthesis Catalysts. Catal. Today 2013, 214, 100-139. https://doi.org/10.1016/j.cattod.2013.05.011.

(5) Schulz, H. Principles of Fischer-Tropsch Synthesis-Constraints on Essential Reactions Ruling FT-Selectivity. Catal. Today 2013, 214, 140-151. https://doi.org/10.1016/j.cattod.2013.05.009.

(6) Aluha, J.; Boahene, P.; Dalai, A.; Hu, Y.; Bere, K.; Braidy, N.; Abatzoglou, N. Synthesis and Characterization of $\mathrm{Co} / \mathrm{C}$ and $\mathrm{Fe} / \mathrm{C}$ Nanocatalysts for FischerTropsch Synthesis: A Comparative Study Using a Fixed-Bed Reactor. Ind. Eng. Chem. Res. 2015, 54 (43), 10661-10674. https://doi.org/10.1021/acs.iecr.5b03003.

(7) Suess, D. L. M.; Peters, J. C. A CO-Derived Iron Dicarbyne That Releases Olefin upon Hydrogenation. J. Am. Chem. Soc. 2013, 135 (34), 12580-12583. https://doi.org/10.1021/ja406874k.

(8) Deegan, M. M.; Peters, J. C. CO Reduction to CH3OSiMe3: Electrophile-Promoted Hydride Migration at a Single Fe Site. J. Am. Chem. Soc. 2017, 139 (7), 25612564. https://doi.org/10.1021/jacs.6b12444.

(9) Reppe, W.; Vetter, H. Carbonylierung VI. Synthesen Mit Metallcarbonylwasserstoffen. Justus Liebigs Ann. Chem. 1953, 582 (1), 133-161. https://doi.org/10.1002/jlac.19535820107.

(10) Massoudi, R.; Kim, J. H.; King, R. B.; King, A. D. Homogeneous Catalysis of the Reppe Reaction with Iron Pentacarbonyl: The Production of Propionaldehyde and 1-Propanol from Ethylene. J. Am. Chem. Soc. 1987, 109 (24), 7428-7433. https://doi.org/10.1021/ja00258a029.

(11) Tamura, M.; Kochi, J. K. Vinylation of Grignard Reagents. Catalysis by Iron. J. Am. Chem. Soc. 1971, 93 (6), 1487-1489. https://doi.org/10.1021/ja00735a030.

(12) Smith, R. S.; Kochi, J. K. Mechanistic Studies of Iron Catalysis in the Cross Coupling of Alkenyl Halides and Grignard Reagents. J Org Chem 1976, 41 (3), 502-509.

(13) Kochi, J. K. Organometallic Mechanisms and Catalysis - 1st Edition, 1st Ed.; Academic Press, 1979.

(14) Poulos, T. L. Heme Enzyme Structure and Function. Chem. Rev. 2014, 114 (7), 3919-3962. https://doi.org/10.1021/cr400415k.

(15) Grau, M.; Britovsek, G. J. P. High-Valent Iron in Biomimetic Alkane Oxidation Catalysis. In Iron Catalysis II; Bauer, E., Ed.; Topics in Organometallic Chemistry; 
Springer International Publishing: Cham, 2015; pp 145-171. https://doi.org/10.1007/3418_2015_100.

(16) Shaik, S.; Straganz, G. D.; Munro, A. W.; Sen, S.; Mowat, C.; Nam, W.; Derat, E.; Bugg, T.; Proshlyakov, D. A.; Hausinger, R. P. Iron-Containing Enzymes: Versatile Catalysts of Hydroxylation Reactions in Nature; Royal Society of Chemistry: Cambridge, UNITED KINGDOM, 2011.

(17) Baranovskiy, A. G.; Siebler, H. M.; Pavlov, Y. I.; Tahirov, T. H. Chapter One - IronSulfur Clusters in DNA Polymerases and Primases of Eukaryotes. In Methods in Enzymology; David, S. S., Ed.; Fe-S Cluster Enzymes Part B; Academic Press, 2018; Vol. 599, pp 1-20. https://doi.org/10.1016/bs.mie.2017.09.003.

(18) Ryle, M. J.; Koehntop, K. D.; Liu, A.; Que, L.; Hausinger, R. P. Interconversion of Two Oxidized Forms of Taurine/a-Ketoglutarate Dioxygenase, a Non-Heme Iron Hydroxylase: Evidence for Bicarbonate Binding. Proc. Natl. Acad. Sci. 2003, 100 (7), 3790-3795. https://doi.org/10.1073/pnas.0636740100.

(19) Vavon, G.; Mottez, P. Action of Halogenated Derivatives on Aromatic Magnesium Halides in the Presence of FeCl3. J. Röntgen Soc. 1944, 218, 557-559.

(20) Tamao, K.; Sumitani, K.; Kumada, M. Selective Carbon-Carbon Bond Formation by Cross-Coupling of Grignard Reagents with Organic Halides. Catalysis by NickelPhosphine Complexes. J. Am. Chem. Soc. 1972, 94 (12), 4374-4376. https://doi.org/10.1021/ja00767a075.

(21) Corriu, R. J. P.; Masse, J. P. Activation of Grignard Reagents by Transition-Metal Complexes. A New and Simple Synthesis of Trans-Stilbenes and Polyphenyls. J. Chem. Soc. Chem. Commun. 1972, No. 3, 144a-144a. https://doi.org/10.1039/C3972000144A.

(22) Yamamura, M.; Moritani, I.; Murahashi, S.-I. The Reaction of $\sigma$-Vinylpalladium Complexes with Alkyllithiums. Stereospecific Syntheses of Olefins from Vinyl Halides and Alkyllithiums. J. Organomet. Chem. 1975, 91 (2), C39-C42. https://doi.org/10.1016/S0022-328X(00)89636-9.

(23) Baba, S.; Negishi, E. A Novel Stereospecific Alkenyl-Alkenyl Cross-Coupling by a Palladium- or Nickel-Catalyzed Reaction of Alkenylalanes with Alkenyl Halides. J. Am. Chem. Soc. 1976, 98 (21), 6729-6731. https://doi.org/10.1021/ja00437a067.

(24) Dohle, W.; Kopp, F.; Cahiez, G.; Knochel, P. Fe(III)-Catalyzed Cross-Coupling Between Functionalized Arylmagnesium Compounds and Alkenyl Halides. Synlett 2001, 2001 (12), 1901-1904. https://doi.org/10.1055/s-2001-18748.

(25) Aleandri, L. E.; Bogdanović, B. The Magnesium Route to Active Metals and Intermetallics https://onlinelibrary.wiley.com/doi/abs/10.1002/9783527615179.ch08 (accessed Aug 6, 2019). https://doi.org/10.1002/9783527615179.ch08.

(26) Muñoz, S. B.; Daifuku, S. L.; Sears, J. D.; Baker, T. M.; Carpenter, S. H.; Brennessel, W. W.; Neidig, M. L. The N-Methylpyrrolidone (NMP) Effect in IronCatalyzed Cross-Coupling with Simple Ferric Salts and MeMgBr. Angew. Chem. Int. Ed. 2018, 57 (22), 6496-6500. https://doi.org/10.1002/anie.201802087.

(27) Fürstner, A.; Leitner, A.; Méndez, M.; Krause, H. Iron-Catalyzed Cross-Coupling Reactions. J. Am. Chem. Soc. 2002, 124 (46), 13856-13863. https://doi.org/10.1021/ja027190t. 
(28) Nakamura, M.; Matsuo, K.; Ito, S.; Nakamura, E. Iron-Catalyzed Cross-Coupling of Primary and Secondary Alkyl Halides with Aryl Grignard Reagents. J. Am. Chem. Soc. 2004, 126 (12), 3686-3687. https://doi.org/10.1021/ja049744t.

(29) Bedford, R. B.; Bruce, D. W.; Frost, R. M.; Hird, M. Simple Iron-Amine Catalysts for the Cross-Coupling of Aryl Grignards with Alkyl Halides Bearing $\beta$-Hydrogens. Chem. Commun. 2005, $O$ (33), 4161-4163. https://doi.org/10.1039/B507133J.

(30) Sherry, B. D.; Fürstner, A. The Promise and Challenge of Iron-Catalyzed Cross Coupling. Acc. Chem. Res. 2008, 41 (11), 1500-1511. https://doi.org/10.1021/ar800039x.

(31) Czaplik, W. M.; Mayer, M.; Jacobi von Wangelin, A. Domino Iron Catalysis: Direct Aryl-Alkyl Cross-Coupling. Angew. Chem. Int. Ed. 2009, 48 (3), 607-610. https://doi.org/10.1002/anie.200804434.

(32) Kleimark, J.; Hedström, A.; Larsson, P.-F.; Johansson, C.; Norrby, P.-O. Mechanistic Investigation of Iron-Catalyzed Coupling Reactions. ChemCatChem 2009, 1 (1), 152-161. https://doi.org/10.1002/cctc.200900061.

(33) Noda, D.; Sunada, Y.; Hatakeyama, T.; Nakamura, M.; Nagashima, H. Effect of TMEDA on Iron-Catalyzed Coupling Reactions of ArMgX with Alkyl Halides. J. Am. Chem. Soc. 2009, 131 (17), 6078-6079. https://doi.org/10.1021/ja901262g.

(34) Gao, H.; Yan, C.; Tao, X.-P.; Xia, Y.; Sun, H.-M.; Shen, Q.; Zhang, Y. Synthesis of Anionic Iron(II) Complex Bearing an N-Heterocyclic Carbene Ligand and Its Catalysis for Aryl Grignard Cross-Coupling of Alkyl Halides. Organometallics 2010, 29 (18), 4189-4192. https://doi.org/10.1021/om100482w.

(35) Kleimark, J.; Larsson, P.-F.; Emamy, P.; Hedström, A.; Norrby, P.-O. Low Temperature Studies of Iron-Catalyzed Cross-Coupling of Alkyl Grignard Reagents with Aryl Electrophiles. Adv. Synth. Catal. 2012, 354 (2-3), 448-456. https://doi.org/10.1002/adsc.201100392.

(36) Guisán-Ceinos, M.; Tato, F.; Buñuel, E.; Calle, P.; Cárdenas, D. J. Fe-Catalysed Kumada-Type Alkyl-Alkyl Cross-Coupling. Evidence for the Intermediacy of Fe(I) Complexes. Chem. Sci. 2013, 4 (3), 1098-1104. https://doi.org/10.1039/C2SC21754F.

(37) Xia, C.-L.; Xie, C.-F.; Wu, Y.-F.; Sun, H.-M.; Shen, Q.; Zhang, Y. Efficient CrossCoupling of Aryl Grignard Reagents with Alkyl Halides by Recyclable Ionic Iron(III) Complexes Bearing a Bis(Phenol)-Functionalized Benzimidazolium Cation. Org. Biomol. Chem. 2013, 11 (46), 8135-8144. https://doi.org/10.1039/C3OB41376D.

(38) Chua, Y.-Y.; Duong, H. A. Selective Kumada Biaryl Cross-Coupling Reaction Enabled by an Iron(lii) Alkoxide-N-Heterocyclic Carbene Catalyst System. Chem. Commun. 2014, 50 (61), 8424. https://doi.org/10.1039/c4cc02930e.

(39) Bauer, G.; Wodrich, M. D.; Scopelliti, R.; Hu, X. Iron Pincer Complexes as Catalysts and Intermediates in Alkyl-Aryl Kumada Coupling Reactions. Organometallics 2015, 34 (1), 289-298. https://doi.org/10.1021/om501122p.

(40) Bedford, R. B. How Low Does Iron Go? Chasing the Active Species in FeCatalyzed Cross-Coupling Reactions. Acc. Chem. Res. 2015, 48 (5), 1485-1493. https://doi.org/10.1021/acs.accounts.5b00042.

(41) Bekhradnia, A.; Norrby, P.-O. New Insights into the Mechanism of Iron-Catalyzed Cross-Coupling Reactions. Dalton Trans. 2015, 44 (9), 3959-3962. https://doi.org/10.1039/C4DT03491K. 
(42) Hedström, A.; Izakian, Z.; Vreto, I.; Wallentin, C.-J.; Norrby, P.-O. On the Radical Nature of Iron-Catalyzed Cross-Coupling Reactions. Chem. - Eur. J. 2015, 21 (15), 5946-5953. https://doi.org/10.1002/chem.201406096.

(43) Przyojski, J. A.; Veggeberg, K. P.; Arman, H. D.; Tonzetich, Z. J. Mechanistic Studies of Catalytic Carbon-Carbon Cross-Coupling by Well-Defined Iron NHC Complexes. ACS Catal. 2015, 5 (10), 5938-5946. https://doi.org/10.1021/acscatal.5b01445.

(44) Cassani, C.; Bergonzini, G.; Wallentin, C.-J. Active Species and Mechanistic Pathways in Iron-Catalyzed C-C Bond-Forming Cross-Coupling Reactions. ACS Catal. 2016, 6 (3), 1640-1648. https://doi.org/10.1021/acscatal.5b02441.

(45) Guérinot, A.; Cossy, J. Iron-Catalyzed C-C Cross-Couplings Using Organometallics. Top. Curr. Chem. 2016, 374 (4). https://doi.org/10.1007/s41061016-0047-x.

(46) Toriyama, F.; Cornella, J.; Wimmer, L.; Chen, T.-G.; Dixon, D. D.; Creech, G.; Baran, P. S. Redox-Active Esters in Fe-Catalyzed C-C Coupling. J. Am. Chem. Soc. 2016, 138 (35), 11132-11135. https://doi.org/10.1021/jacs.6b07172.

(47) Carpenter, S. H.; Neidig, M. L. A Physical-Inorganic Approach for the Elucidation of Active Iron Species and Mechanism in Iron-Catalyzed Cross-Coupling. Isr. J. Chem. 2017, 57 (12), 1106-1116. https://doi.org/10.1002/ijch.201700036.

(48) Parchomyk, T.; Koszinowski, K. Iron-Catalyzed Cross-Coupling: Mechanistic Insight for Rational Applications in Synthesis. Synthesis 2017, 49 (15), 3269-3280. https://doi.org/10.1055/s-0036-1588428.

(49) Sears, J. D.; Neate, P. G. N.; Neidig, M. L. Intermediates and Mechanism in IronCatalyzed Cross-Coupling. J. Am. Chem. Soc. 2018, 140 (38), 11872-11883. https://doi.org/10.1021/jacs.8b06893.

(50) Neidig, M. L.; Carpenter, S. H.; Curran, D. J.; DeMuth, J. C.; Fleischauer, V. E.; Iannuzzi, T. E.; Neate, P. G. N.; Sears, J. D.; Wolford, N. J. Development and Evolution of Mechanistic Understanding in Iron-Catalyzed Cross-Coupling. Acc. Chem. Res. 2019, 52 (1), 140-150. https://doi.org/10.1021/acs.accounts.8b00519.

(51) Piontek, A.; Bisz, E.; Szostak, M. Iron-Catalyzed Cross-Couplings in the Synthesis of Pharmaceuticals: In Pursuit of Sustainability. Angew. Chem. Int. Ed. 2018, 57 (35), 11116-11128. https://doi.org/10.1002/anie.201800364.

(52) Cossee, P. Ziegler-Natta Catalysis I. Mechanism of Polymerization of a-Olefins with Ziegler-Natta Catalysts. J. Catal. 1964, 3 (1), 80-88. https://doi.org/10.1016/0021-9517(64)90095-8.

(53) Schrock, R. R. Living Ring-Opening Metathesis Polymerization Catalyzed by WellCharacterized Transition-Metal Alkylidene Complexes. Acc. Chem. Res. 1990, 23 (5), 158-165. https://doi.org/10.1021/ar00173a007.

(54) Spaleck, W.; Kueber, F.; Winter, A.; Rohrmann, J.; Bachmann, B.; Antberg, M.; Dolle, V.; Paulus, E. F. The Influence of Aromatic Substituents on the Polymerization Behavior of Bridged Zirconocene Catalysts. Organometallics 1994, 13 (3), 954-963. https://doi.org/10.1021/om00015a032.

(55) Brintzinger, H. H.; Fischer, D.; Mülhaupt, R.; Rieger, B.; Waymouth, R. M. Stereospecific Olefin Polymerization with Chiral Metallocene Catalysts. Angew. Chem. Int. Ed. Engl. 1995, 34 (11), 1143-1170. https://doi.org/10.1002/anie.199511431. 
(56) Johnson, L. K.; Killian, C. M.; Brookhart, M. New Pd(II)- and Ni(II)-Based Catalysts for Polymerization of Ethylene and .Alpha.-Olefins. J. Am. Chem. Soc. 1995, 117 (23), 6414-6415. https://doi.org/10.1021/ja00128a054.

(57) Johnson, L. K.; Mecking, S.; Brookhart, M. Copolymerization of Ethylene and Propylene with Functionalized Vinyl Monomers by Palladium(II) Catalysts. J. Am. Chem. Soc. 1996, 118 (1), 267-268. https://doi.org/10.1021/ja953247i.

(58) Killian, C. M.; Tempel, D. J.; Johnson, L. K.; Brookhart, M. Living Polymerization of a-Olefins Using Nill-a-Diimine Catalysts. Synthesis of New Block Polymers Based on a-Olefins. J. Am. Chem. Soc. 1996, 118 (46), 11664-11665. https://doi.org/10.1021/ja962516h.

(59) Mecking, S.; Johnson, L. K.; Wang, L.; Brookhart, M. Mechanistic Studies of the Palladium-Catalyzed Copolymerization of Ethylene and a-Olefins with Methyl Acrylate. J. Am. Chem. Soc. 1998, 120 (5), 888-899. https://doi.org/10.1021/ja964144i.

(60) Small, B. L.; Brookhart, M.; Bennett, A. M. A. Highly Active Iron and Cobalt Catalysts for the Polymerization of Ethylene. J. Am. Chem. Soc. 1998, 120 (16), 4049-4050. https://doi.org/10.1021/ja9802100.

(61) Britovsek, G. J. P.; Gibson, V. C.; Kimberley, B. S.; Maddox, P. J.; Mctavish, S. J.; Solan, G. A.; White, J. P.; Williams, D. J. Novel Olefin Polymerization Catalysts Based on Iron and Cobalt from Iron and Cobalt. Chem Commun 1998, 311 (1), 849-850.

(62) Britovsek, G. J. P.; Bruce, M.; Gibson, V. C.; Kimberley, B. S.; Maddox, P. J.; Mastroianni, S.; McTavish, S. J.; Redshaw, C.; Solan, G. A.; Strömberg, S.; et al. Iron and Cobalt Ethylene Polymerization Catalysts Bearing 2,6-Bis(Imino)Pyridyl Ligands: Synthesis, Structures, and Polymerization Studies. J. Am. Chem. Soc. 1999, 121 (38), 8728-8740. https://doi.org/10.1021/ja990449w.

(63) Deng, L.; Margl, P.; Ziegler, T. Mechanistic Aspects of Ethylene Polymerization by Iron(II)-Bisimine Pyridine Catalysts: A Combined Density Functional Theory and Molecular Mechanics Study. J. Am. Chem. Soc. 1999, 121 (27), 6479-6487. https://doi.org/10.1021/ja984385l.

(64) Talsi, E. P.; Babushkin, D. E.; Semikolenova, N. V.; Zudin, V. N.; Zakharov, V. A. Ethylene Polymerization in the Presence of Iron(II) 2,6-Bis(Imine)Pyridine Complex: Structures of Key Intermediates. Kinet. Catal. 2001, 42 (2), 147-153. https://doi.org/10.1023/A:1010451213400.

(65) Britovsek, G. J. P.; Clentsmith, G. K. B.; Gibson, V. C.; Goodgame, D. M. L.; McTavish, S. J.; Pankhurst, Q. A. The Nature of the Active Site in Bis(Imino)Pyridine Iron Ethylene Polymerisation Catalysts. Catal. Commun. 2002, 3 (5), 207-211. https://doi.org/10.1016/S1566-7367(02)00092-4.

(66) Britovsek, G. J. P.; Gibson, V. C.; Spitzmesser, S. K.; Tellmann, K. P.; White, A. J. P.; Williams, D. J. Cationic 2,6-Bis(Imino)Pyridine Iron and Cobalt Complexes: Synthesis, Structures, Ethylene Polymerisation and Ethylene/Polar Monomer CoPolymerisation Studies. J. Chem. Soc. Dalton Trans. 2002, No. 6, 1159. https://doi.org/10.1039/b106614p.

(67) Chen, Y.; Chen, R.; Qian, C.; Dong, X.; Sun, J. Halogen-Substituted 2,6Bis(Imino)Pyridyl Iron and Cobalt Complexes: Highly Active Catalysts for 
Polymerization and Oligomerization of Ethylene. Organometallics 2003, 22 (21), 4312-4321. https://doi.org/10.1021/om0302894.

(68) Bryliakov, K. P.; Semikolenova, N. V.; Zakharov, V. A.; Talsi, E. P. Active Intermediates of Ethylene Polymerization over 2,6-Bis(Imino)Pyridyl Iron Complex Activated with Aluminum Trialkyls and Methylaluminoxane. Organometallics 2004, 23 (22), 5375-5378. https://doi.org/10.1021/om0497184.

(69) Bryliakov, K. P.; Semikolenova, N. V.; Zudin, V. N.; Zakharov, V. A.; Talsi, E. P. Ferrous Rather than Ferric Species Are the Active Sites in Bis(Imino)Pyridine Iron Ethylene Polymerization Catalysts. Catal. Commun. 2004, 5 (1), 45-48. https://doi.org/10.1016/j.catcom.2003.11.010.

(70) Bouwkamp, M. W.; Lobkovsky, E.; Chirik, P. J. Bis(Imino)Pyridine Iron(II) Alkyl Cations for Olefin Polymerization. J. Am. Chem. Soc. 2005, 127 (27), 9660-9661. https://doi.org/10.1021/ja0524447.

(71) Cámpora, J.; Naz, A. M.; Palma, P.; Álvarez, E.; Reyes, M. L. 2,6-Diiminopyridine Iron(II) Dialkyl Complexes. Interaction with Aluminum Alkyls and Ethylene Polymerization Catalysis. Organometallics 2005, 24 (21), 4878-4881. https://doi.org/10.1021/om050645d.

(72) van Meurs, M.; Britovsek, G. J. P.; Gibson, V. C.; Cohen, S. A. Polyethylene Chain Growth on Zinc Catalyzed by Olefin Polymerization Catalysts: A Comparative Investigation of Highly Active Catalyst Systems across the Transition Series. J. Am. Chem. Soc. 2005, 127 (27), 9913-9923. https://doi.org/10.1021/ja050100a.

(73) Nakayama, Y.; Saito, J.; Bando, H.; Fujita, T. MgCl2/R'nAl(OR)3-n: An Excellent Activator/Support for Transition-Metal Complexes for Olefin Polymerization. Chem. - Eur. J. 2006, 12 (29), 7546-7556. https://doi.org/10.1002/chem.200600355.

(74) Huang, R.; Koning, C. E.; Chadwick, J. C. Effects of Hydrogen in Ethylene Polymerization and Oligomerization with Magnesium Chloride-Supported Bis(Imino)Pyridyl Iron Catalysts. J. Polym. Sci. Part Polym. Chem. 2007, 45 (17), 4054-4061. https://doi.org/10.1002/pola.22159.

(75) Talsi, E. P.; Bryliakov, K. P.; Semikolenova, N. V.; Zakharov, V. A.; Bochmann, M. Key Intermediates in Metallocene-and Post-Metallocene-Catalyzed Polymerization. Kinet. Catal. 2007, 48 (4), 490-504. https://doi.org/10.1134/S0023158407040052.

(76) Bryliakov, K. P.; Talsi, E. P.; Semikolenova, N. V.; Zakharov, V. A. Formation and Nature of the Active Sites in Bis(Imino)Pyridine Iron-Based Polymerization Catalysts. Organometallics 2009, 28 (11), 3225-3232. https://doi.org/10.1021/om8010905.

(77) Semikolenova, N. V.; Zakharov, V. A.; Echevskaja, L. G.; Matsko, M. A.; Bryliakov, K. P.; Talsi, E. P. Homogeneous Catalysts for Ethylene Polymerization Based on Bis(Imino)Pyridine Complexes of Iron, Cobalt, Vanadium and Chromium. Catal. Today 2009, 144 (3-4), 334-340. https://doi.org/10.1016/j.cattod.2009.01.022.

(78) Tondreau, A. M.; Milsmann, C.; Patrick, A. D.; Hoyt, H. M.; Lobkovsky, E.; Wieghardt, K.; Chirik, P. J. Synthesis and Electronic Structure of Cationic, Neutral, and Anionic Bis(Imino)Pyridine Iron Alkyl Complexes: Evaluation of Redox Activity in Single-Component Ethylene Polymerization Catalysts. J. Am. Chem. Soc. 2010, 132 (42), 15046-15059. https://doi.org/10.1021/ja106575b. 
(79) Minaev, B.; Baryshnikova, A.; Sun, W.-H. Spin-Dependent Effects in Ethylene Polymerization with Bis(Imino)Pyridine Iron(II) Complexes. J. Organomet. Chem. 2016, 811, 48-65. https://doi.org/10.1016/j.jorganchem.2016.03.020.

(80) Kato, M.; Kamigaito, M.; Sawamoto, M.; Higashimura, T. Polymerization of Methyl Methacrylate with the Carbon Tetrachloride/Dichlorotris-

(Triphenylphosphine)Ruthenium(II)/Methylaluminum Bis(2,6-Di-TertButylphenoxide) Initiating System: Possibility of Living Radical Polymerization. Macromolecules 1995, 28 (5), 1721-1723. https://doi.org/10.1021/ma00109a056.

(81) Wang, J.-S.; Matyjaszewski, K. Controlled/"Living" Radical Polymerization. Halogen Atom Transfer Radical Polymerization Promoted by a $\mathrm{Cu}(\mathrm{I}) / \mathrm{Cu}(\mathrm{II})$ Redox Process. Macromolecules 1995, 28 (23), 7901-7910. https://doi.org/10.1021/ma00127a042.

(82) Ando, T.; Kato, M.; Kamigaito, M.; Sawamoto, M. Living Radical Polymerization of Methyl Methacrylate with Ruthenium Complex: Formation of Polymers with Controlled Molecular Weights and Very Narrow Distributions. Macromolecules 1996, 29 (3), 1070-1072. https://doi.org/10.1021/ma951175+.

(83) Patten, T. E.; Xia, J.; Abernathy, T.; Matyjaszewski, K. Polymers with Very Low Polydispersities from Atom Transfer Radical Polymerization. Science 1996, 272 (5263), 866-868. https://doi.org/10.1126/science.272.5263.866.

(84) Matyjaszewski, K.; Patten, T. E.; Xia, J. Controlled/“Living” Radical Polymerization. Kinetics of the Homogeneous Atom Transfer Radical Polymerization of Styrene. J. Am. Chem. Soc. 1997, 119 (4), 674-680. https://doi.org/10.1021/ja963361g.

(85) Matyjaszewski, K.; Wei, M.; Xia, J.; McDermott, N. E. Controlled/“Living” Radical Polymerization of Styrene and Methyl Methacrylate Catalyzed by Iron Complexes. Macromolecules 1997, 30 (26), 8161-8164. https://doi.org/10.1021/ma971010w.

(86) Fechete, I. Paul Sabatier - The Father of the Chemical Theory of Catalysis. Comptes Rendus Chim. 2016, 19. https://doi.org/10.1016/j.crci.2016.08.006.

(87) Luo, J.; G. Oliver, A.; McIndoe, J. S. A Detailed Kinetic Analysis of RhodiumCatalyzed Alkyne Hydrogenation. Dalton Trans. 2013, 42 (31), 11312-11318. https://doi.org/10.1039/C3DT51212F.

(88) Spessard, G.; Miessler, G. Organometallic Chemistry, 3rd Ed.; Oxford Press, 2016.

(89) Zell, T.; Langer, R. From Ruthenium to Iron and Manganese-A Mechanistic View on Challenges and Design Principles of Base-Metal Hydrogenation Catalysts https://onlinelibrary.wiley.com/doi/abs/10.1002/cctc.201701722 (accessed Aug 20, 2019). https://doi.org/10.1002/cctc.201701722.

(90) Frankel, E. N.; Emken, E. A.; Peters, H. M.; Davison, V. L.; Butterfield, R. O. Homogeneous Hydrogenation of Methyl Linoleate Catalyzed by Iron Pentacarbonyl. Characterization of Methyl Octadecadienoate-Iron Tricarbonyl Complexes ${ }^{1}$. J. Org. Chem. 1964, 29 (11), 3292-3297. https://doi.org/10.1021/jo01034a040.

(91) Stern, R.; Sajus, L. New Homogeneous Cobalt Catalysts for Hydrogenation. Tetrahedron Lett. 1968, 9 (60), 6313-6314. https://doi.org/10.1016/S00404039(00)75462-1.

(92) Harmon, R. E.; Gupta, S. K.; Brown, D. J. Hydrogenation of Organic Compounds Using Homogeneous Catalysts. Chem. Rev. 1973, 73 (1), 21-52. https://doi.org/10.1021/cr60281a003. 
(93) Miller, M. E.; Grant, E. R. Gas-Phase Organometallic Catalysis: Kinetics and Mechanism of the Hydrogenation of Ethylene by $\mathrm{Fe}(\mathrm{CO}) 3(\mathrm{C} 2 \mathrm{H} 4) 2$. J. Am. Chem. Soc. 1987, 109 (26), 7951-7960. https://doi.org/10.1021/ja00260a004.

(94) Bianchini, C.; Meli, A.; Peruzzini, M.; Frediani, P.; Bohanna, C.; Esteruelas, M. A.; Oro, L. A. Selective Hydrogenation of 1-Alkynes to Alkenes Catalyzed by an Iron(II) Cis-Hydride .Eta.2-Dihydrogen Complex. A Case of Intramolecular Reaction between .Eta.2-H2 and .Sigma.-Vinyl Ligands. Organometallics 1992, 11 (1), 138145. https://doi.org/10.1021/om00037a029.

(95) Bart, S. C.; Lobkovsky, E.; Chirik, P. J. Preparation and Molecular and Electronic Structures of Iron(0) Dinitrogen and Silane Complexes and Their Application to Catalytic Hydrogenation and Hydrosilation. J. Am. Chem. Soc. 2004, 126 (42), 13794-13807. https://doi.org/10.1021/ja046753t.

(96) Archer, A. M.; Bouwkamp, M. W.; Cortez, M.-P.; Lobkovsky, E.; Chirik, P. J. Arene Coordination in Bis(Imino)Pyridine Iron Complexes: Identification of Catalyst Deactivation Pathways in Iron-Catalyzed Hydrogenation and Hydrosilation. Organometallics 2006, 25 (18), 4269-4278. https://doi.org/10.1021/om060441c.

(97) Enthaler, S.; Erre, G.; Tse, M. K.; Junge, K.; Beller, M. Biomimetic Transfer Hydrogenation of Ketones with Iron Porphyrin Catalysts. Tetrahedron Lett. 2006, 47 (46), 8095-8099. https://doi.org/10.1016/j.tetlet.2006.09.058.

(98) Casey, C. P.; Guan, H. An Efficient and Chemoselective Iron Catalyst for the Hydrogenation of Ketones. J. Am. Chem. Soc. 2007, 129 (18), 5816-5817. https://doi.org/10.1021/ja071159f.

(99) Bullock, R. M. An Iron Catalyst for Ketone Hydrogenations under Mild Conditions. Angew. Chem. Int. Ed. 2007, 46 (39), 7360-7363. https://doi.org/10.1002/anie.200703053.

(100) Sui-Seng, C.; Freutel, F.; Lough, A. J.; Morris, R. H. Highly Efficient Catalyst Systems Using Iron Complexes with a Tetradentate PNNP Ligand for the Asymmetric Hydrogenation of Polar Bonds. Angew. Chem. Int. Ed. 2008, 47 (5), 940-943. https://doi.org/10.1002/anie.200705115.

(101) Ziebart, C.; Federsel, C.; Anbarasan, P.; Jackstell, R.; Baumann, W.; Spannenberg, A.; Beller, M. Well-Defined Iron Catalyst for Improved Hydrogenation of Carbon Dioxide and Bicarbonate. J. Am. Chem. Soc. 2012, 134 (51), 2070120704. https://doi.org/10.1021/ja307924a.

(102) Langer, R.; Iron, M. A.; Konstantinovski, L.; Diskin-Posner, Y.; Leitus, G.; BenDavid, Y.; Milstein, D. Iron Borohydride Pincer Complexes for the Efficient Hydrogenation of Ketones under Mild, Base-Free Conditions: Synthesis and Mechanistic Insight. Chem. - Eur. J. 2012, 18 (23), 7196-7209. https://doi.org/10.1002/chem.201200159.

(103) Wang, D.; Astruc, D. The Golden Age of Transfer Hydrogenation. Chem. Rev. 2015, 115 (13), 6621-6686. https://doi.org/10.1021/acs.chemrev.5b00203.

(104) Bauer, G.; Hu, X. Recent Developments of Iron Pincer Complexes for Catalytic Applications. Inorg. Chem. Front. 2016, 3 (6), 741-765. https://doi.org/10.1039/C5QI00262A.

(105) Chakraborty, S.; Leitus, G.; Milstein, D. Selective Hydrogenation of Nitriles to Primary Amines Catalyzed by a Novel Iron Complex. Chem. Commun. 2016, 52 (9), 1812-1815. https://doi.org/10.1039/C5CC08204H. 
(106) Gorgas, N.; Stöger, B.; Veiros, L. F.; Kirchner, K. Highly Efficient and Selective Hydrogenation of Aldehydes: A Well-Defined Fe(II) Catalyst Exhibits Noble-Metal Activity. ACS Catal. 2016, 6 (4), 2664-2672. https://doi.org/10.1021/acscatal.6b00436.

(107) Rezayee, N. M.; Samblanet, D. C.; Sanford, M. S. Iron-Catalyzed Hydrogenation of Amides to Alcohols and Amines. ACS Catal. 2016, 6 (10), 6377-6383. https://doi.org/10.1021/acscatal.6b01454.

(108) Xu, R.; Chakraborty, S.; Bellows, S. M.; Yuan, H.; Cundari, T. R.; Jones, W. D. Iron-Catalyzed Homogeneous Hydrogenation of Alkenes under Mild Conditions by a Stepwise, Bifunctional Mechanism. ACS Catal. 2016, 6 (3), 2127-2135. https://doi.org/10.1021/acscatal.5b02674.

(109) Docherty, J. H.; Peng, J.; Dominey, A. P.; Thomas, S. P. Activation and Discovery of Earth-Abundant Metal Catalysts Using Sodium Tert-Butoxide. Nat. Chem. 2017, 9 (6), 595-600. https://doi.org/10.1038/nchem.2697.

(110) Espinal-Viguri, M.; Neale, S. E.; Coles, N. T.; Macgregor, S. A.; Webster, R. L. Room Temperature Iron-Catalyzed Transfer Hydrogenation and Regioselective Deuteration of Carbon-Carbon Double Bonds. J. Am. Chem. Soc. 2019, 141 (1), 572-582. https://doi.org/10.1021/jacs.8b11553.

(111) Tondreau, A. M.; Atienza, C. C. H.; Darmon, J. M.; Milsmann, C.; Hoyt, H. M.; Weller, K. J.; Nye, S. A.; Lewis, K. M.; Boyer, J.; Delis, J. G. P.; et al. Synthesis, Electronic Structure, and Alkene Hydrosilylation Activity of Terpyridine and Bis(Imino)Pyridine Iron Dialkyl Complexes. Organometallics 2012, 31 (13), 48864893. https://doi.org/10.1021/om3004527.

(112) Tondreau, A. M.; Atienza, C. C. H.; Weller, K. J.; Nye, S. A.; Lewis, K. M.; Delis, J. G. P.; Chirik, P. J. Iron Catalysts for Selective Anti-Markovnikov Alkene Hydrosilylation Using Tertiary Silanes. Science 2012, 335 (6068), 567-570. https://doi.org/10.1126/science.1214451.

(113) Peng, D.; Zhang, Y.; Du, X.; Zhang, L.; Leng, X.; Walter, M. D.; Huang, Z. Phosphinite-Iminopyridine Iron Catalysts for Chemoselective Alkene Hydrosilylation. J. Am. Chem. Soc. 2013, 135 (51), 19154-19166. https://doi.org/10.1021/ja404963f.

(114) Greenhalgh, M. D.; Frank, D. J.; Thomas, S. P. Iron-Catalysed Chemo-, Regio-, and Stereoselective Hydrosilylation of Alkenes and Alkynes Using a Bench-Stable Iron(II) Pre-Catalyst. Adv. Synth. Catal. 2014, 356 (2-3), 584-590. https://doi.org/10.1002/adsc.201300827.

(115) Chen, J.; Cheng, B.; Cao, M.; Lu, Z. Iron-Catalyzed Asymmetric Hydrosilylation of 1,1-Disubstituted Alkenes. Angew. Chem. Int. Ed. 2015, 54 (15), 4661-4664. https://doi.org/10.1002/anie.201411884.

(116) Challinor, A. J.; Calin, M.; Nichol, G. S.; Carter, N. B.; Thomas, S. P. AmineActivated Iron Catalysis: Air- and Moisture-Stable Alkene and Alkyne Hydrofunctionalization. Adv. Synth. Catal. 2016, 358 (15), 2404-2409. https://doi.org/10.1002/adsc.201600570.

(117) Carney, J. R.; Dillon, B. R.; Campbell, L.; Thomas, S. P. Manganese-Catalyzed Hydrofunctionalization of Alkenes. Angew. Chem. Int. Ed. 2018, 57 (33), 1062010624. https://doi.org/10.1002/anie.201805483. 
(118) Obligacion, J. V.; Chirik, P. J. Earth-Abundant Transition Metal Catalysts for Alkene Hydrosilylation and Hydroboration: Opportunities and Assessments. Nat. Rev. Chem. 2018, 2 (5), 15-34. https://doi.org/10.1038/s41570-018-0001-2.

(119) Troegel, D.; Stohrer, J. Recent Advances and Actual Challenges in Late Transition Metal Catalyzed Hydrosilylation of Olefins from an Industrial Point of View. Coord. Chem. Rev. 2011, 255 (13), 1440-1459. https://doi.org/10.1016/j.ccr.2010.12.025.

(120) Hu, M.-Y.; He, Q.; Fan, S.-J.; Wang, Z.-C.; Liu, L.-Y.; Mu, Y.-J.; Peng, Q.; Zhu, S.-F. Ligands with 1,10-Phenanthroline Scaffold for Highly Regioselective IronCatalyzed Alkene Hydrosilylation. Nat. Commun. 2018, 9 (1), 221. https://doi.org/10.1038/s41467-017-02472-6.

(121) Zhang, L.; Cheng, J.; Hou, Z. Highly Efficient Catalytic Hydrosilylation of Carbon Dioxide by an N-Heterocyclic Carbene Copper Catalyst. Chem. Commun. 2013, 49 (42), 4782. https://doi.org/10.1039/c3cc41838c.

(122) Darcel, C.; Sortais, J.-B. Iron-Catalyzed Reduction and Hydroelementation Reactions. In Iron Catalysis II; Bauer, E., Ed.; Topics in Organometallic Chemistry; Springer International Publishing: Cham, 2015; pp 173-216. https://doi.org/10.1007/3418_2015_104.

(123) Jia, X.; Huang, Z. Conversion of Alkanes to Linear Alkylsilanes Using an IridiumIron-Catalysed Tandem Dehydrogenation-Isomerization-Hydrosilylation. Nat. Chem. 2016, 8 (2), 157-161. https://doi.org/10.1038/nchem.2417.

(124) Du, X.; Huang, Z. Advances in Base-Metal-Catalyzed Alkene Hydrosilylation. ACS Catal. 2017, 7 (2), 1227-1243. https://doi.org/10.1021/acscatal.6b02990.

(125) Männig, D.; Nöth, H. Catalytic Hydroboration with Rhodium Complexes. Angew. Chem. Int. Ed. Engl. 1985, 24 (10), 878-879.

https://doi.org/10.1002/anie.198508781.

(126) Evans, D. A.; Fu, G. C. The Rhodium-Catalyzed Hydroboration of Olefins: A Mechanistic Investigation. J. Org. Chem. 1990, 55 (8), 2280-2282. https://doi.org/10.1021/jo00295a007.

(127) Burgess, Kevin.; Ohlmeyer, M. J. Transition-Metal Promoted Hydroborations of Alkenes, Emerging Methodology for Organic Transformations. Chem. Rev. 1991, 91 (6), 1179-1191. https://doi.org/10.1021/cr00006a003.

(128) Obligacion, J. V.; Chirik, P. J. Highly Selective Bis(Imino)Pyridine Iron-Catalyzed Alkene Hydroboration. Org. Lett. 2013, 15 (11), 2680-2683. https://doi.org/10.1021/ol400990u.

(129) Greenhalgh, M. D.; Thomas, S. P. Chemo-, Regio-, and Stereoselective IronCatalysed Hydroboration of Alkenes and Alkynes. Chem. Commun. 2013, 49 (95), 11230-11232. https://doi.org/10.1039/C3CC46727A.

(130) Ruddy, A. J.; Sydora, O. L.; Small, B. L.; Stradiotto, M.; Turculet, L. (NPhosphinoamidinate)Cobalt-Catalyzed Hydroboration: Alkene Isomerization Affords Terminal Selectivity. Chem. - Eur. J. 2014, 20 (43), 13918-13922. https://doi.org/10.1002/chem.201403945.

(131) Tseng, K.-N. T.; Kampf, J. W.; Szymczak, N. K. Regulation of Iron-Catalyzed Olefin Hydroboration by Ligand Modifications at a Remote Site. ACS Catal. 2015, 5 (1), 411-415. https://doi.org/10.1021/cs501820w. 
(132) Scheuermann, M. L.; Johnson, E. J.; Chirik, P. J. Alkene Isomerization Hydroboration Promoted by Phosphine- Ligated Cobalt Catalysts. Org. Lett. 2015, 17, 2716-2719. https://doi.org/10.1021/acs.orglett.5b01135.

(133) Liu, Y.; Zhou, Y.; Wang, H.; Qu, J. FeCl2-Catalyzed Hydroboration of Aryl Alkenes with Bis(Pinacolato)Diboron. RSC Adv. 2015, 5 (90), 73705-73713. https://doi.org/10.1039/C5RA14869C.

(134) Iwamoto, H.; Kubota, K.; Ito, H. Highly Selective Markovnikov Hydroboration of Alkyl-Substituted Terminal Alkenes with a Phosphine-Copper(I) Catalyst. Chem. Commun. 2016, 52 (35), 5916-5919. https://doi.org/10.1039/C6CC00782A.

(135) Espinal-Viguri, M.; Woof, C. R.; Webster, R. L. Iron-Catalyzed Hydroboration: Unlocking Reactivity through Ligand Modulation. Chem. - Eur. J. 2016, 22 (33), 11605-11608. https://doi.org/10.1002/chem.201602818.

(136) MacNair, A. J.; Millet, C. R. P.; Nichol, G. S.; Ironmonger, A.; Thomas, S. P. Markovnikov-Selective, Activator-Free Iron-Catalyzed Vinylarene Hydroboration. ACS Catal. 2016, 6 (10), 7217-7221. https://doi.org/10.1021/acscatal.6b02281.

(137) Agahi, R.; Challinor, A. J.; Carter, N. B.; Thomas, S. P. Earth-Abundant Metal Catalysis Enabled by Counterion Activation. Org. Lett. 2019, 21 (4), 993-997. https://doi.org/10.1021/acs.orglett.8b03986.

(138) Cruz, T. F. C.; Pereira, L. C. J.; Waerenborgh, J. C.; Veiros, L. F.; Gomes, P. T. Hydroboration of Terminal Olefins with Pinacolborane Catalyzed by New 2Iminopyrrolyl Iron( II ) Complexes. Catal. Sci. Technol. 2019, 9 (13), 3347-3360. https://doi.org/10.1039/C8CY02319K.

(139) Huehls, C. B.; Lin, A.; Yang, J. Iron-Catalyzed Intermolecular Hydroamination of Styrenes. Org. Lett. 2014, 16 (14), 3620-3623. https://doi.org/10.1021/ol5013907.

(140) Ma, W.; Zhang, X.; Fan, J.; Liu, Y.; Tang, W.; Xue, D.; Li, C.; Xiao, J.; Wang, C. Iron-Catalyzed Anti-Markovnikov Hydroamination and Hydroamidation of Allylic Alcohols. J. Am. Chem. Soc. 2019, 141 (34), 13506-13515. https://doi.org/10.1021/jacs.9b05221.

(141) Marcyk, P. T.; Cook, S. P. Iron-Catalyzed Hydroamination and Hydroetherification of Unactivated Alkenes. Org. Lett. 2019, 21 (5), 1547-1550. https://doi.org/10.1021/acs.orglett.9b00427.

(142) Zhang, Y.; Huang, C.; Lin, X.; Hu, Q.; Hu, B.; Zhou, Y.; Zhu, G. Modular Synthesis of Alkylarylazo Compounds via Iron(III)-Catalyzed Olefin Hydroamination. Org. Lett. 2019, 21 (7), 2261-2264. https://doi.org/10.1021/acs.orglett.9b00540.

(143) Osborn, J. A.; Wilkinson, G.; Young, J. F. Mild Hydroformylation of Olefins Using Rhodium Catalysts. Chem. Commun. Lond. 1965, No. 2, 17. https://doi.org/10.1039/c19650000017.

(144) Palágyi, J.; Markó, L. Hydroformylation and Hydrogenation of Styrene with Carbon Monoxide and Water Catalyzed by Iron Carbonyls. J. Organomet. Chem. 1982, 236 (3), 343-347. https://doi.org/10.1016/S0022-328X(00)86908-9.

(145) Sakai, N.; Mano, S.; Nozaki, K.; Takaya, H. Highly Enantioselective Hydroformylation of Olefins Catalyzed by New Phosphine Phosphite-Rhodium(I) Complexes. J. Am. Chem. Soc. 1993, 115 (15), 7033-7034. https://doi.org/10.1021/ja00068a095. 
(146) Nozaki, K.; Sakai, N.; Nanno, T.; Higashijima, T.; Mano, S.; Horiuchi, T.; Takaya, $\mathrm{H}$. Highly Enantioselective Hydroformylation of Olefins Catalyzed by Rhodium(I) Complexes of New Chiral Phosphine-Phosphite Ligands. J. Am. Chem. Soc. 1997, 119 (19), 4413-4423. https://doi.org/10.1021/ja970049d.

(147) Breschi, C.; Piparo, L.; Pertici, P.; Maria Caporusso, A.; Vitulli, G. (H6Cyclohepta-1,3,5-Triene)(H4-Cycloocta-1,5-Diene)Iron(0) Complex as Attractive Precursor in Catalysis. J. Organomet. Chem. 2000, 607 (1), 57-63. https://doi.org/10.1016/S0022-328X(00)00211-4.

(148) Brezny, A. C.; Landis, C. R. Unexpected CO Dependencies, Catalyst Speciation, and Single Turnover Hydrogenolysis Studies of Hydroformylation via High Pressure NMR Spectroscopy. J. Am. Chem. Soc. 2017, 139 (7), 2778-2785. https://doi.org/10.1021/jacs.6b12533.

(149) Pandey, S.; Raj, K. V.; Shinde, D. R.; Vanka, K.; Kashyap, V.; Kurungot, S.; Vinod, C. P.; Chikkali, S. H. Iron Catalyzed Hydroformylation of Alkenes under Mild Conditions: Evidence of an Fe(II) Catalyzed Process. J. Am. Chem. Soc. 2018, 140 (12), 4430-4439. https://doi.org/10.1021/jacs.8b01286.

(150) Saito, S.; Nakagawa, S.; Koizumi, T.; Hirayama, K.; Yamamoto, Y. NickelMediated Regio- and Chemoselective Carboxylation of Alkynes in the Presence of Carbon Dioxide. J. Org. Chem. 1999, 64 (11), 3975-3978. https://doi.org/10.1021/jo982443f.

(151) Williams, C. M.; Johnson, J. B.; Rovis, T. Nickel-Catalyzed Reductive Carboxylation of Styrenes Using CO 2. J. Am. Chem. Soc. 2008, 130 (45), 1493614937. https://doi.org/10.1021/ja8062925.

(152) Li, S.; Yuan, W.; Ma, S. Highly Regio- and Stereoselective Three-Component Nickel-Catalyzed Syn-Hydrocarboxylation of Alkynes with Diethyl Zinc and Carbon Dioxide. Angew. Chem. - Int. Ed. 2011, 50 (11), 2578-2582. https://doi.org/10.1002/anie.201007128.

(153) Greenhalgh, M. D.; Thomas, S. P. Iron-Catalyzed, Highly Regioselective Synthesis of a-Aryl Carboxylic Acids from Styrene Derivatives and CO 2. J. Am. Chem. Soc. 2012, 134 (29), 11900-11903. https://doi.org/10.1021/ja3045053.

(154) Ostapowicz, T. G.; Schmitz, M.; Krystof, M.; Klankermayer, J.; Leitner, W. Carbon Dioxide as a C1 Building Block for the Formation of Carboxylic Acids by Formal Catalytic Hydrocarboxylation. Angew. Chem. Int. Ed. 2013, 52 (46), 1211912123. https://doi.org/10.1002/anie.201304529.

(155) Huguet, N.; Jevtovikj, I.; Gordillo, A.; Lejkowski, M. L.; Lindner, R.; Bru, M.; Khalimon, A. Y.; Rominger, F.; Schunk, S. A.; Hofmann, P.; et al. Nickel-Catalyzed Direct Carboxylation of Olefins with CO2: One-Pot Synthesis of $\alpha, \beta-$ Unsaturated Carboxylic Acid Salts. Chem. - Eur. J. 2014, 20 (51), 16858-16862. https://doi.org/10.1002/chem.201405528.

(156) Santhoshkumar, R.; Hong, Y.-C.; Luo, C.-Z.; Wu, Y.-C.; Hung, C.-H.; Hwang, K.Y.; Tu, A.-P.; Cheng, C.-H. Synthesis of Vinyl Carboxylic Acids Using Carbon Dioxide as a Carbon Source by Iron-Catalyzed Hydromagnesiation. ChemCatChem 2016, 8 (13), 2210-2213. https://doi.org/10.1002/cctc.201600279.

(157) Shao, P.; Wang, S.; Chen, C.; Xi, C. Cp $2 \mathrm{TiCl}_{2}$-Catalyzed Regioselective Hydrocarboxylation of Alkenes with $\mathrm{CO}_{2}$. Org. Lett. 2016, 18 (9), 2050-2053. https://doi.org/10.1021/acs.orglett.6b00665. 
(158) Gaydou, M.; Moragas, T.; Juliá-Hernández, F.; Martin, R. Site-Selective Catalytic Carboxylation of Unsaturated Hydrocarbons with $\mathrm{CO} 2$ and Water. J. Am. Chem. Soc. 2017, 139 (35), 12161-12164. https://doi.org/10.1021/jacs.7b07637.

(159) Liu, W.; Ren, W.; Li, J.; Shi, Y.; Chang, W.; Shi, Y. A Ligand-Directed Catalytic Regioselective Hydrocarboxylation of Aryl Olefins with Pd and Formic Acid. Org. Lett. 2017, 19 (7), 1748-1751. https://doi.org/10.1021/acs.orglett.7b00507.

(160) Seo, H.; Liu, A.; Jamison, T. F. Direct $\beta$-Selective Hydrocarboxylation of Styrenes with $\mathrm{CO}_{2}$ Enabled by Continuous Flow Photoredox Catalysis. J. Am. Chem. Soc. 2017, 139 (40), 13969-13972. https://doi.org/10.1021/jacs.7b05942.

(161) Zhang, L.; Hou, Z. N-Heterocyclic Carbene (NHC)-Copper-Catalysed Transformations of Carbon Dioxide. Chem. Sci. 2013, 4 (9), 3395. https://doi.org/10.1039/c3sc51070k.

(162) Luan, Y.-X.; Ye, M. Transition Metal-Mediated or Catalyzed Hydrocarboxylation of Olefins with CO2. Tetrahedron Lett. 2018, 59 (10), 853-861. https://doi.org/10.1016/j.tetlet.2018.01.035.

(163) Meng, Q.-Y.; Wang, S.; Huff, G. S.; König, B. Ligand-Controlled Regioselective Hydrocarboxylation of Styrenes with $\mathrm{CO}_{2}$ by Combining Visible Light and Nickel Catalysis. J. Am. Chem. Soc. 2018, 140 (9), 3198-3201. https://doi.org/10.1021/jacs.7b13448.

(164) Butcher, T. W.; McClain, E. J.; Hamilton, T. G.; Perrone, T. M.; Kroner, K. M.; Donohoe, G. C.; Akhmedov, N. G.; Petersen, J. L.; Popp, B. V. Regioselective Copper-Catalyzed Boracarboxylation of Vinyl Arenes. Org. Lett. 2016, 18 (24), 6428-6431. https://doi.org/10.1021/acs.orglett.6b03326.

(165) Li, S.; Ma, S. Quadri-Synergetic Effect for Highly Effective Carbon Dioxide Fixation and Its Application to Indoloquinolinone. Adv. Synth. Catal. 2012, 354 (13), 2387-2394. https://doi.org/10.1002/adsc.201200469.

(166) Fujihara, T.; Tani, Y.; Semba, K.; Terao, J.; Tsuji, Y. Copper-Catalyzed Silacarboxylation of Internal Alkynes by Employing Carbon Dioxide and Silylboranes. Angew. Chem. Int. Ed. 2012, 51 (46), 11487-11490. https://doi.org/10.1002/anie.201207148.

(167) Miao, B.; Li, S.; Li, G.; Ma, S. Cyclic Anti-Azacarboxylation of 2-Alkynylanilines with Carbon Dioxide. Org. Lett. 2016, 18 (11), 2556-2559. https://doi.org/10.1021/acs.orglett.6b00884.

(168) Speier, J. L.; Webster, J. A.; Barnes, G. H. The Addition of Silicon Hydrides to Olefinic Double Bonds. Part II. The Use of Group VIII Metal Catalysts. J. Am. Chem. Soc. 1957, 79 (4), 974-979. https://doi.org/10.1021/ja01561a054.

(169) Nakajima, Y.; Shimada, S. Hydrosilylation Reaction of Olefins: Recent Advances and Perspectives. RSC Adv. 2015, 5 (26), 20603-20616. https://doi.org/10.1039/C4RA17281G.

(170) Greenhalgh, M. Iron-Catalysed Hydrofunctionalisation of Alkenes and Alkynes; Springer Theses; Springer International Publishing: Cham, 2016. https://doi.org/10.1007/978-3-319-33663-3.

(171) Brown, H. C. Boranes in Organic Chemistry**Based upon the Roger Adams Award Address to the Division of Organic Chemistry of the American Chemical Society in Ann Arbor, Michigan, June 15, 1971. In Advances in Organometallic 
Chemistry; Stone, F. G. A., West, R., Eds.; Academic Press, 1973; Vol. 11, pp 120. https://doi.org/10.1016/S0065-3055(08)60158-6.

(172) Liu, X.; Hartwig, J. F. Palladium-Catalyzed Arylation of Trimethylsilyl Enolates of Esters and Imides. High Functional Group Tolerance and Stereoselective Synthesis of a-Aryl Carboxylic Acid Derivatives. J. Am. Chem. Soc. 2004, 126 (16), 5182-5191. https://doi.org/10.1021/ja031544e.

(173) Evano, G. Synthesis from Alkenes (Excluding Reactions with Carboxylic Acid Derivatives); Georg Thieme Verlag KG, 2007; p 1. https://doi.org/10.1055/sos-sd020-00172.

(174) Dai, X.; Strotman, N. A.; Fu, G. C. Catalytic Asymmetric Hiyama Cross-Couplings of Racemic a-Bromo Esters. J. Am. Chem. Soc. 2008, 130 (11), 3302-3303. https://doi.org/10.1021/ja8009428.

(175) Meng, Q.-Y.; Wang, S.; Huff, G. S.; König, B. Ligand-Controlled Regioselective Hydrocarboxylation of Styrenes with $\mathrm{CO} 2$ by Combining Visible Light and Nickel Catalysis. J. Am. Chem. Soc. 2018, 140 (9), 3198-3201. https://doi.org/10.1021/jacs.7b13448.

(176) Greenhalgh, M. D.; Thomas, S. P. Iron-Catalyzed, Highly Regioselective Synthesis of a-Aryl Carboxylic Acids from Styrene Derivatives and $\mathrm{CO}_{2}$. J. Am. Chem. Soc. 2012, 134 (29), 11900-11903. https://doi.org/10.1021/ja3045053.

(177) Wang, X.; Nakajima, M.; Martin, R. Ni-Catalyzed Regioselective Hydrocarboxylation of Alkynes with $\mathrm{CO} 2$ by Using Simple Alcohols as Proton Sources. J. Am. Chem. Soc. 2015, 137 (28), 8924-8927. https://doi.org/10.1021/jacs.5b05513.

(178) Williamson, K. S.; Yoon, T. P. Iron-Catalyzed Aminohydroxylation of Olefins. J. Am. Chem. Soc. 2010, 132 (13), 4570-4571. https://doi.org/10.1021/ja1013536.

(179) Yang, Y.; Song, R.-J.; Ouyang, X.-H.; Wang, C.-Y.; Li, J.-H.; Luo, S. IronCatalyzed Intermolecular 1,2-Difunctionalization of Styrenes and Conjugated Alkenes with Silanes and Nucleophiles. Angew. Chem. Int. Ed. 2017, 56 (27), 7916-7919. https://doi.org/10.1002/anie.201702349.

(180) Liu, W.; Li, Y.; Liu, K.; Li, Z. Iron-Catalyzed Carbonylation-Peroxidation of Alkenes with Aldehydes and Hydroperoxides. J. Am. Chem. Soc. 2011, 133 (28), 10756-10759. https://doi.org/10.1021/ja204226n.

(181) Shen, T.; Yuan, Y.; Song, S.; Jiao, N. Iron-Catalyzed Aerobic Difunctionalization of Alkenes: A Highly Efficient Approach to Construct Oxindoles by $\mathrm{C}-\mathrm{S}$ and $\mathrm{C}-\mathrm{C}$ Bond Formation. Chem. Commun. 2014, 50 (31), 4115-4118. https://doi.org/10.1039/C4CC00401A.

(182) Lu, D.-F.; Zhu, C.-L.; Jia, Z.-X.; Xu, H. Iron(II)-Catalyzed Intermolecular AminoOxygenation of Olefins through the $\mathrm{N}-\mathrm{O}$ Bond Cleavage of Functionalized Hydroxylamines. J. Am. Chem. Soc. 2014, 136 (38), 13186-13189. https://doi.org/10.1021/ja508057u.

(183) Jian, W.; Ge, L.; Jiao, Y.; Qian, B.; Bao, H. Iron-Catalyzed Decarboxylative Alkyl Etherification of Vinylarenes with Aliphatic Acids as the Alkyl Source. Angew.

Chem. Int. Ed. 2017, 56 (13), 3650-3654. https://doi.org/10.1002/anie.201612365.

(184) Finkbeiner, H.; Cooper, G. Titanium-Catalyzed Isomerization and OlefinExchange Reactions of Alkylmagnesium Halides: A Novel Method for Preparation of the Grignard Reagent. J Am Chem Soc 1961, 26, 4779-4780. 
(185) Sato, F.; Urabe, H. Hydromagnesiation of Alkenes and Alkynes. Grignard Reag. New Dev. 2000.

(186) Shirakawa, E.; Ikeda, D.; Yamaguchi, S.; Hayashi, T. Fe-Cu Cooperative Catalysis in the Isomerization of Alkyl Grignard Reagents. Chem. Commun. 2008, No. 10, 1214. https://doi.org/10.1039/b717717h.

(187) Shirakawa, E.; Ikeda, D.; Masui, S.; Yoshida, M.; Hayashi, T. Iron-Copper Cooperative Catalysis in the Reactions of Alkyl Grignard Reagents: Exchange Reaction with Alkenes and Carbometalation of Alkynes. J. Am. Chem. Soc. 2012, 134 (1), 272-279. https://doi.org/10.1021/ja206745w.

(188) Greenhalgh, M. D.; Kolodziej, A.; Sinclair, F.; Thomas, S. P. Iron-Catalyzed Hydromagnesiation: Synthesis and Characterization of Benzylic Grignard Reagent Intermediate and Application in the Synthesis of Ibuprofen. Organometallics 2014, 33 (20), 5811-5819. https://doi.org/10.1021/om500319h.

(189) Gao, Y.; Urabe, H.; Sato, F. First Practical Hydrozincation of Dienes Catalyzed by Cp2TiCl2. Generation of Allylzinc Reagent and Its Reaction with Carbonyl Compounds. J. Org. Chem. 1994, 59 (19), 5521-5523. https://doi.org/10.1021/jo00098a005.

(190) Gao, Y.; Harada, K.; Hata, T.; Urabe, H.; Sato, F. Stereo- and Regioselective Generation of Alkenylzinc Reagents via Titanium-Catalyzed Hydrozincation of Internal Acetylenes. J. Org. Chem. 1995, 60 (2), 290-291. https://doi.org/10.1021/jo00107a003.

(191) Klement, I.; Lütjens, H.; Knochel, P. Oxidation of Zinc Organometallics Prepared by Hydrozincation or Carbozincation Using Oxygen. Tetrahedron Lett. 1995, 36 (18), 3161-3164. https://doi.org/10.1016/0040-4039(95)00501-3.

(192) Vettel, S.; Vaupel, A.; Knochel, P. A New Preparation of Diorganozincs from Olefins via a Nickel Catalyzed Hydrozincation. Tetrahedron Lett. 1995, 36 (7), 1023-1026. https://doi.org/10.1016/0040-4039(94)02438-H.

(193) Jones, A. S.; Paliga, J. F.; Greenhalgh, M. D.; Quibell, J. M.; Steven, A.; Thomas, S. P. Broad Scope Hydrofunctionalization of Styrene Derivatives Using IronCatalyzed Hydromagnesiation. Org. Lett. 2014, 16 (22), 5964-5967. https://doi.org/10.1021/ol5029892.

(194) Ren, Q.; Wu, N.; Cai, Y.; Fang, J. DFT Study of the Mechanisms of IronCatalyzed Regioselective Synthesis of a-Aryl Carboxylic Acids from Styrene Derivatives and CO 2. Organometallics 2016, 35 (23), 3932-3938. https://doi.org/10.1021/acs.organomet.6b00681.

(195) Neate, P.; Greenhalgh, M. D.; Brennessel, W. W.; Thomas, S. P.; Neidig, M. L. Mechanism of the Bis(Imino)Pyridine Iron-Catalyzed Hydromagnesiation of Styrene Derivatives. J. Am. Chem. Soc. 2019, jacs.9b04869. https://doi.org/10.1021/jacs.9b04869.

(196) Hoops, S.; Gauges, R.; Lee, C.; Pahle, J.; Simus, N.; Singhal, M.; Xu, L.; Mendes, P.; Kummer, U. COPASI - A COmplex PAthway SImulator. Bioinformatics 2006, 22 (24), 3067-3074. https://doi.org/10.1093/bioinformatics/btl485.

(197) Bakac, A.; Won, T.-J.; Espenson, J. H. Novel Pathways in the Reactions of Superoxometal Complexes. Inorg. Chem. 1996, 35 (8), 2171-2175. https://doi.org/10.1021/ic951144v. 
(198) Steinhoff, B. A.; Guzei, I. A.; Stahl, S. S. Mechanistic Characterization of Aerobic Alcohol Oxidation Catalyzed by $\mathrm{Pd}(\mathrm{OAc}) 2$ /Pyridine Including Identification of the Catalyst Resting State and the Origin of Nonlinear [Catalyst] Dependence. J. Am. Chem. Soc. 2004, 126 (36), 11268-11278. https://doi.org/10.1021/ja049962m.

(199) Wile, B. M.; Trovitch, R. J.; Bart, S. C.; Tondreau, A. M.; Lobkovsky, E.; Milsmann, C.; Bill, E.; Wieghardt, K.; Chirik, P. J. Reduction Chemistry of Aryl- and Alkyl-Substituted Bis(Imino)Pyridine Iron Dihalide Compounds: Molecular and Electronic Structures of [(PDI) 2 Fe] Derivatives. Inorg. Chem. 2009, 48 (9), 41904200. https://doi.org/10.1021/ic801623m.

(200) Shejwalkar, P.; Rath, N. P.; Bauer, E. B. New Bis(Imino)Pyridine Complexes of Iron(II) and Iron(III), and Their Catalytic- Activity in the Mukaiyama Aldol Reaction. Synthesis 2014, 46 (01), 57-66. https://doi.org/10.1055/s-0033-1340081.

(201) Cornish-Bowden, A. Principles of Enzyme Kinetics; Elsevier, 2014.

(202) Bart, S. C.; Chłopek, K.; Bill, E.; Bouwkamp, M. W.; Lobkovsky, E.; Neese, F.; Wieghardt, K.; Chirik, P. J. Electronic Structure of Bis(Imino)Pyridine Iron Dichloride, Monochloride, and Neutral Ligand Complexes: A Combined Structural, Spectroscopic, and Computational Study. J. Am. Chem. Soc. 2006, 128 (42), 13901-13912. https://doi.org/10.1021/ja064557b.

(203) Tondreau, A. M.; Stieber, S. C. E.; Milsmann, C.; Lobkovsky, E.; Weyhermüller, T.; Semproni, S. P.; Chirik, P. J. Oxidation and Reduction of Bis(Imino)Pyridine Iron Dinitrogen Complexes: Evidence for Formation of a Chelate Trianion. Inorg. Chem. 2013, 52 (2), 635-646. https://doi.org/10.1021/ic301675t.

(204) Casitas, A.; Krause, H.; Goddard, R.; Fürstner, A. Elementary Steps of Iron Catalysis: Exploring the Links between Iron Alkyl and Iron Olefin Complexes for Their Relevance in $\mathrm{C} \square \mathrm{H}$ Activation and $\mathrm{C} \square \mathrm{C}$ Bond Formation. Angew. Chem. Int. Ed. 2015, 54 (5), 1521-1526. https://doi.org/10.1002/anie.201410069.

(205) Jr, K. P.; Böhm, S.; Braunerová, G.; Waisser, K.; Exner, O. Reaction Series Not Obeying the Hammett Equation: Conformational Equilibria of Substituted Thiobenzanilides. New J. Chem. 2002, 26 (7), 861-866. https://doi.org/10.1039/B111156F.

(206) Ravi, R.; Sanjeev, R.; Jagannadham, V. Nonlinear Taft Polar Free Energy Relationship: Reactions of N-Substituted Benzyl Amines with Benzyl Bromide in Methanol. Int. J. Chem. Kinet. 2013, 45 (12), 803-810. https://doi.org/10.1002/kin.20818.

(207) Hansch, Corwin.; Leo, A.; Taft, R. W. A Survey of Hammett Substituent Constants and Resonance and Field Parameters. Chem. Rev. 1991, 91 (2), 165195. https://doi.org/10.1021/cr00002a004.

(208) Frisch, M. J.; Trucks, G. W.; Schlegel, H. B.; Scuseria, G. E.; Robb, M. A.; Cheeseman, J. R.; Scalmani, G.; Barone, V.; Petersson, G. A.; Nakatsuji, H.; et al. Gaussian 16 Revision C.01; Guassian Inc: Wallingford CT, 2016.

(209) Glendening, E. D.; Reed, A. E.; Carpenter, J. E.; Weinhold, F. NBO; TCl: University of Wisconsin Madison, 1998.

(210) Abu-Surrah, A. S.; Lappalainen, K.; Piironen, U.; Lehmus, P.; Repo, T.; Leskelä, M. New Bis(Imino)Pyridine-Iron(II)- and Cobalt(II)-Based Catalysts: Synthesis, Characterization and Activity towards Polymerization of Ethylene. J. Organomet. Chem. 2002, 648 (1-2), 55-61. https://doi.org/10.1016/S0022-328X(01)01418-8. 
(211) Li, L.; Gomes, P. T. Oligomerization and Polymerization of Olefins with Iron and Cobalt Catalysts Containing 2,6-Bis(Imino)Pyridine and Related Ligands. In Olefin Upgrading Catalysis by Nitrogen-based Metal Complexes II: State of the art and Perspectives; CAMPORA, J., GIAMBASTIANI, G., Eds.; Catalysis by Metal Complexes; Springer Netherlands: Dordrecht, 2011; pp 77-197. https://doi.org/10.1007/978-94-007-0696-5_3.

(212) Small, B. L.; Brookhart, M.; Bennett, A. M. A. Highly Active Iron and Cobalt Catalysts for the Polymerization of Ethylene. J. Am. Chem. Soc. 1998, 120 (16), 4049-4050. https://doi.org/10.1021/ja9802100.

(213) Britovsek, G. J. P.; Clentsmith, G. K. B.; Gibson, V. C.; Goodgame, D. M. L.; Mctavish, S. J.; Pankhurst, Q. a. The Nature of the Active Site in Bis (Imino ) Pyridine Iron Ethylene Polymerisation Catalysts. Catal. Commun. 2002, 3 (5), 207211. http://dx.doi.org/10.1016/S1566-7367(02)00092-4.

(214) Greenhalgh, M. D.; Thomas, S. P. Iron-Catalyzed Hydromagnesiation of Olefins. Synlett 2013, 24 (5), 531-534. https://doi.org/10.1055/s-0032-1318075.

(215) Greenhalgh, M. D.; Jones, A. S.; Thomas, S. P. Iron-Catalysed Hydrofunctionalisation of Alkenes and Alkynes. ChemCatChem 2015, 7 (2), 190 222. https://doi.org/10.1002/cctc.201402693.

(216) Greenhalgh, M. D. Iron-Catalysed Hydrofunctionalization of Alkenes and Alkynes; 2016.

(217) Sahle, S.; Gauges, R.; Kummer, U.; Lee, C.; Mendes, P.; Tech, V. SIMULATION OF BIOCHEMICAL NETWORKS USING COPASI - A COMPLEX PATHWAY SIMULATOR. Proc. 2006 Winter Simul. Conf. 2006, 1698-1706.

(218) Ranganathan, P.; Pramesh, C. S.; Aggarwal, R. Common Pitfalls in Statistical Analysis: Measures of Agreement. Perspect. Clin. Res. 2017, 8 (4), 187-191. https://doi.org/10.4103/picr.PICR_123_17. 